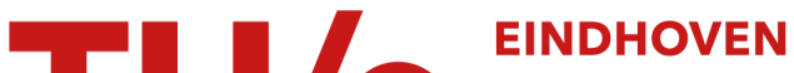 \\ UNIVERSITY OF \\ TECHNOLOGY
}

\section{Designing sculpting light systems for information decoration}

\section{Citation for published version (APA):}

Wu, J. (2020). Designing sculpting light systems for information decoration. [Phd Thesis 1 (Research TU/e / Graduation TU/e), Industrial Design]. Technische Universiteit Eindhoven.

Document status and date:

Published: $10 / 06 / 2020$

\section{Document Version:}

Publisher's PDF, also known as Version of Record (includes final page, issue and volume numbers)

\section{Please check the document version of this publication:}

- A submitted manuscript is the version of the article upon submission and before peer-review. There can be important differences between the submitted version and the official published version of record. People interested in the research are advised to contact the author for the final version of the publication, or visit the $\mathrm{DOI}$ to the publisher's website.

- The final author version and the galley proof are versions of the publication after peer review.

- The final published version features the final layout of the paper including the volume, issue and page numbers.

Link to publication

\section{General rights}

Copyright and moral rights for the publications made accessible in the public portal are retained by the authors and/or other copyright owners and it is a condition of accessing publications that users recognise and abide by the legal requirements associated with these rights.

- Users may download and print one copy of any publication from the public portal for the purpose of private study or research.

- You may not further distribute the material or use it for any profit-making activity or commercial gain

- You may freely distribute the URL identifying the publication in the public portal.

If the publication is distributed under the terms of Article 25fa of the Dutch Copyright Act, indicated by the "Taverne" license above, please follow below link for the End User Agreement:

www.tue.nl/taverne

Take down policy

If you believe that this document breaches copyright please contact us at:

openaccess@tue.nl

providing details and we will investigate your claim. 


$$
\begin{aligned}
& \text { Designing } \\
& \text { Sculpting } \\
& \text { Light } \\
& \text { Systems } \\
& \text { for } \\
& \text { Information } \\
& \text { Decoration }
\end{aligned}
$$



Designing Sculpting Light Systems for

\section{Information Decoration}

Doctoral Dissertation by Jiang Wu 


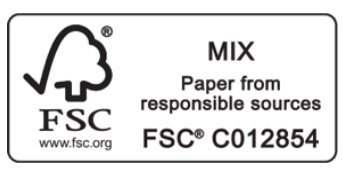

A catalogue record is available from the Eindhoven University of Technology Library ISBN: 978-90-386-5053-1

\section{(c) Jiang Wu, 2020}

All rights reserved. No part of this book may be reproduced or transmitted in any form or by any means, electronic or mechanical, including photocopying, recording, or by any information storage and retrieval system without permission of the author. 


\title{
Designing Sculpting Light Systems for Information Decoration
}

\author{
PROEFSCHRIFT
}

ter verkrijging van de graad van doctor aan de Technische Universiteit Eindhoven, op gezag van de rector magnificus prof.dr.ir. F.P.T. Baaijens, voor een commissie aangewezen door het College voor Promoties, in het openbaar te verdedigen op woensdag 10 juni 2020 om 11:00 uur

door

Jiang Wu

geboren te Henan, China 
Dit proefschrift is goedgekeurd door de promotoren en de samenstelling van de promotiecommissie is als volgt:

$\begin{array}{ll}\begin{array}{ll}\text { voorzitter: } \\ \text { 1e promotor: }\end{array} & \text { prof.dr. L. Chen } \\ \text { copromotor(en): } & \text { prof.dr.ir. J.H. Eggen } \\ \text { leden: } & \text { dr.ir. H.A. van Essen } \\ & \text { dr. M. Graves-Petersen (Aarhus University) } \\ & \text { prof.dr. S. Luo (Zhejiang University) } \\ & \text { prof. A.A.J. van Berlo } \\ \text { adviseur(s): } & \text { dr. K. van Mensvoort }\end{array}$

Het onderzoek of ontwerp dat in dit proefschrift wordt beschreven is uitgevoerd in overeenstemming met de TU/e Gedragscode Wetenschapsbeoefening. 


\section{Table of Contents}

CHAPTER 1. Introduction 1

1.1 Research Scope 3

1.1.1 Ubiquitous Computing and Tangible Bits 4

1.1.2 Calm Technology and Ambient Displays 4

1.1.3 Information Decoration and Kinetic Light Sculptures 5

1.1.4 Shape-changing techniques 6

$\begin{array}{lll}1.1 .5 & \text { Research focus }\end{array}$

1.2 Related Work 8

1.2.1 Augmenting shape-changing with light 8

1.2.2 Sculpting light with shape-changing 9

$\begin{array}{lll}1.2 .3 & \text { Discussion } & 11\end{array}$

$\begin{array}{lll}1.2 .4 & \text { Research goal } & 12\end{array}$

$1.4 \quad$ Research Questions and Approach 12

1.5 Thesis overview 14

CHAPTER 2. Design Explorations $\quad 16$

2.1 Design Goals 17

$2.2 \quad$ First Design Cycle: Timer Lamp 19

2.2.1 Design concept 19

2.2.2 Design implementation 19

2.2.3 User feedback 21

2.2.4 Reflection on Design challenges 22

2.3 Second Design Cycle: Timer Shade 23

2.3.1 Design concept 23

2.3.2 Design implementation 24

2.3.3 User feedback 26

$\begin{array}{lll}\text { 2.3.4 Reflections on Design challenges } & 27\end{array}$

2.4 Means to address SLS design challenges 28 
2.4.1 Analysis of relevant state-of-art examples of SLS 28

2.4.2 Overview of Architecture of SLS 29

$\begin{array}{lll}2.5 & \text { Conclusion } & 29\end{array}$

CHAPTER 3. SLS Building Blocks 32

3.1 A Review of the Design Space of SLS 33

3.1.1 Sample for analysis 33

3.1.2 Light sources \& physical objects 34

3.1.3 Manipulations 38

$\begin{array}{lll}3.1 .4 & \text { Attributes } & 40\end{array}$

3.1.5 Creating light patterns $\quad 45$

3.2 Discussion of the Design Space for SLS 47

3.2.1 Information Decoration 48

3.2.2 Manipulations 49

3.2.3 Attributes 50

3.2.4 Light patterns 51

3.2.5 Definition of SLS 54

3.3 An Overview of SLS Building Blocks 54

3.3.1 Four basic components for construction 54

3.3.2 Three types of attributes for manipulation 57

3.3.3 Three kinds of light patterns for information mapping 59

3.4 Four Styles of SLS 61

3.5 Discussion and Conclusion 62

3.5.1 Limitation of the SLS building blocks 62

3.5.2 Potential of using the SLS building blocks 63

$\begin{array}{lll}\text { CHAPTER 4. SLS Framework } & 64\end{array}$

4.1 Intentions 65

4.2 Reflective Clock 66

4.2.1 Highlighting Preset Objects 67

4.2.2 The interaction alters the information representation 68

4.3 Time Glow 68

4.3.1 Distinguishing Passive Objects and Preset Objects 70

$\begin{array}{lll}\text { 4.3.2 The interaction alters the information presentation } & 71\end{array}$ 
4.4 A Framework for SLS

4.5 Designing Adaptive SLS for Information Decoration 74

$\begin{array}{lll}4.6 & \text { Conclusion } & 75\end{array}$

$\begin{array}{lll}\text { CHAPTER 5. SLS Interaction Model } & 76\end{array}$

$\begin{array}{lll}5.1 & \text { Introduction } & 77\end{array}$

5.2 Information Flow 77

5.2.1 The existing information visualisation pipeline 77

5.2.2 Information flow within SLS 78

5.2.3 Design alternatives for the information flow in SLS 84

Table 5.1

$\begin{array}{lll}5.3 & \text { Interaction Model } & 87\end{array}$

$\begin{array}{ll}\text { Figure } 5.9 & 88\end{array}$

5.3.1 Interaction loops 89

5.3.2 The alternative interaction techniques 92

$5.4 \quad$ Using the Interaction Model 94

5.4.1 Describing the existing design concept 95

$\begin{array}{ll}\text { 5.4.2 Discovering new design possibilities } & 97\end{array}$

5.4.3 Envisioning new design concepts 99

5.4.4 Criteria for generating a design concept 106

$\begin{array}{lll}5.4 .5 & 109\end{array}$

$\begin{array}{lll}5.5 \text { Conclusion } & 111\end{array}$

$\begin{array}{lll}\text { CHAPTER 6. SLS Design Guidelines } & 114\end{array}$

6.1 Clues, Ideas, and Concepts 115

6.2 Designing a hypothetical SLS 116

$\begin{array}{ll}6.3 & \text { Design Guidelines } \\ & 117\end{array}$

$\begin{array}{ll}\text { 6.3.1 Collecting clues } & 117\end{array}$

6.3.2 Generating ideas 120

$\begin{array}{ll}\text { 6.3.3 Completing concepts } & 124\end{array}$

6.4 Discussion 128

6.4.1 Potentials of using the SLS Design Guidelines 128

6.4.2 Limitation of using the SLS Design Guidelines 129 
CHAPTER 7. Evaluating the SLS Framework and the SLS Interaction Model 132

7.1 Evaluation Goals and Approach 133

7.2 Evaluation Method 134

$\begin{array}{lll}\text { 7.2.1 Participants } & 134\end{array}$

$\begin{array}{lll}7.2 .2 & \text { Procedure } & 135\end{array}$

$\begin{array}{lll}7.2 .3 & \text { Analysis } & 138\end{array}$

$\begin{array}{lll}7.3 & \text { Results } & 140\end{array}$

$\begin{array}{lll}\text { 7.3.1 } & \text { Results from the Questionnaires } & 140\end{array}$

7.3.2 Results from the Exercises 140

7.3.3 Results from the Comments and Interviews 142

7.4 Findings 146

$\begin{array}{lll}\text { 7.4.1 Understandability } & 146\end{array}$

$\begin{array}{lll}7.4 .2 & \text { Usefulness } & 147\end{array}$

7.4.3 Possible improvements 150

$\begin{array}{lll}\text { 7.4.4 Potential benefits } & 151\end{array}$

7.5 Discussion and Conclusion 153

7.5.1 Limitations of the study 153

7.5.2 Conclusions from the study 153

7.5.3 Recommendations for further development 154

CHAPTER 8. Reflections and Conclusion 156

8.1 Contributions of This Dissertation 157

$\begin{array}{lll}\text { 8.1.1 The design space of SLS } & 157\end{array}$

8.1.2 Systematic approach to designing SLS 160

8.1.3 Design guidelines for SLS 161

8.2 Limitations 162

8.3 Future Work 163

8.3.1 Developing an accessible toolset 163

8.3.2 Generating context-specific insights 163

8.3.3 Inspiring other related fields 164

8.4 Conclusions 166 
References

$\begin{array}{ll}\text { Appendices } & 174\end{array}$

Appendix I: The 33 state-of-the-art examples of SLS 175

Appendix II: The detailed clues, ideas, and concepts 192

Appendix III: The booklet used for evaluation 197

$\begin{array}{lr}\text { About Author } & 214\end{array}$

$\begin{array}{ll}\text { Summary } & 215\end{array}$

$\begin{array}{lr}\text { Acknowledgements } & 216\end{array}$ 
CHAPTER 1.

\section{Introduction}


Light is the prerequisite for us to see the world. Also, light carries information that helps us to make sense of our environments. Nowadays, with the artificial light inundating the world, we are going through an age of "light pollution" (Longcore \& Rich, 2004) and "information overload" (Maes, 2002). In this introduction, we take a historical view of how we human beings use light as an information carrier. Furthermore, we discuss what the new opportunities are of designing light for information communication in a way that could relieve the two issues which we mentioned above.

\section{Natural light}

In ancient times, human beings mastered the skill on how to gain information by observing the natural light. For example, the shadow of the sun presents the time during a day; the sparkling lake shows the breezing wind; the golden sunrise indicates a good weather day. These natural lights are beautiful and subtle, and always calmly stay in our background. As such we appreciate them so much that we even try to bring them inside our architectural spaces in different ways. For instance, several companies (e.g., CoeLux ${ }^{1}$ and Ewinlight ${ }^{2}$ ) are exploring technologies that can simulate skylight. Also, colour changing lamps (e.g., Phillips $\mathrm{Hue}^{3}$ ) are increasingly used in the domestic context to create natural light atmospheres, such as aurora, sunrise and sunset.

\section{Artificial light}

With developments in lighting technology, the use of artificial light sources has been growing fast during the last century. Especially in the past decades, the LED technology has inundated the world with its advantages of efficient, controllable and durable light. Apart from the lighting technology itself, the technologies of electronics, mechanics, computing and materials have developed significantly as well. All of these technologies together leverage the use of artificial light for information communication.

\section{LED displays}

An extreme form of using artificial light sources for information communication is LED displays, on which light is shaped into graphics with a huge number of micro pixelated LEDs. These pixelated lights can display information clearly, accurately and diversely. Moreover,

\footnotetext{
${ }^{1}$ CoeLux. Retrieved on 29.06.2019 from https://www.coelux.com

${ }^{2}$ Ewinlight. Retrieved on 29.06.2019 from http://www.ewinlight.com

${ }^{3}$ Koninklijke Philips N.V (2013). Philips hue. Retrieved on 29.06.2019 from https://www2.meethue.com
} 
these benefits induce people to get immersed in different forms of digital displays, such as the computer, smart phone, and even smart watch.

However, somewhere along the line, we gradually realised that these pervasive informative pixelated lights are competing for our attention, which cause many complaints about "information overload." It impacts our lives mentally as well as physically. Firstly, in this age of information explosion, just placing information on screen will overburden us and will probably cause mental stress. Secondly, just checking information on screen makes us like phubbers, and can causes not only neck injuries but also poor eyesight.

\section{Ambient light systems}

To liberate people from the "information overload," ambient light is considered as a powerful alternative to pixilated light. Ambient light systems (Matviienko et al., 2015) aim to present information that does not always have to stay in the focus attention of people. The current developments in designing ambient light systems show two directions: one is using direct light by manipulating the light sources only; the other employs (indirect) light patterns by manipulation of the physical environment.

\section{Direct light}

Using direct light is the major form of designing ambient light for information communication. In this way, digital information is mapped to the properties of light sources only, regarding colour, brightness, LED position and temporal aspects such as duration and rhythm. For example, Daylight Display (Mankoff \& Dey, 2003) changes its brightness according to the external light conditions; the Ambient Orb $^{4}$ glows in different colours to display real-time stock market trends or traffic congestion.

These examples clearly demonstrate that direct light can convey abstract information without distracting people. However, we are concerned that: designing ambient light by manipulating light sources only is not a long-term solution for the prevention from "information overload." Lighting researchers have shown that future light sources can and will be embedded in walls (Prante et al., 2003), ceilings (Tomitsch, Grechenig, Vande Moere, \& Renan, 2008), floors (Dalton, 2013), furniture (Kaila, Raula, Valtonen, \& Palovuori, 2012) or fabric (Kim, Paulos, \& Gross, 2010). This means that future environments may have a significant number of lights that are occluded by other everyday items (S. A. M. Offermans, van Essen, \& Eggen, 2014). Let us assume that this will come true and think about the future environments lighted by multiple

\footnotetext{
${ }^{4}$ Ambient Devices (2002). Ambient Orb. Retrieved on 29.05.2019 from http://www.ambientdevices.com
} 
types of light sources. By that time, these pervasive light sources will become another kind of "pixelated light" in our environments. It will probably intensify rather than relieve the "information overload." Even worse, the overuse of direct light might cause serious light pollution, which is already taking place in some modern cities nowadays.

\section{Indirect light patterns}

Besides the use of direct light, ambient light also includes different light patterns which present the meta-state of the physical surroundings of the light sources. For example, shadows, reflections, and projections. These light patterns are also able to be used as information carriers. In fact, this idea is not new in the field of arts but only draws little attention in the field of human-computer interaction (HCI). Some examples are Water Lamp (Dahley, Wisneski, \& Ishii, 2003), Heekyoung's transformable lampshade (Jung, Altieri, \& Bardzell, 2010), Fischer's deformable reflector (Hornecker et al., 2015), and Gleamy's transparency variable lamp shade (Cha, Lee, \& Nam, 2016).

These examples show that using light patterns for information communication is more aesthetically pleasing and visually less distracting, compared to using direct light. In this sense, these decorative and meaningful light patterns are fit for (re)presenting the information that can easily stay in the background of people's attention, but that can move to the foreground and be perceived if necessary.

\section{New design space}

To conclude, we believe that meaningful and decorative light patterns will be a desirable alternative to the use of direct light in the future design of ambient light systems. In this way, light sources can be better integrated within their physical environment rather than be rudely embedded just anywhere. More importantly, this approach to present information to people, can be seen as a promising alternative to prevent either "information overload" or "light pollution".

In the remainder of this introduction, we define our research scope by discussing the possible approaches to future ambient lighting systems that are to be built for context-aware environments. Next, we indicate our research focus towards innovative lighting systems that combine lighting control with shape-changing techniques. After that, we discuss the related work and set our research goal particularly in formalizing design methodology. Finally, we define our research questions and describe our research approach. In the end, we introduce the overview of this thesis.

\subsection{Research Scope}


In the previous section, we opened up a new design space that uses decorative meaningful light patterns for (ambient) information communication. To further scope this design space, we discuss the relevant research topics regarding Ubiquitous Computing (Weiser, 2007), Tangible Bits (Ishii, 2004); Calm Technology (Weiser \& Brown, 1995), Ambient Displays (Yarin et al., 2007), Information Decoration (Eggen \& Van Mensvoort, 2009), and Shapechanging techniques (Coelho \& Zigelbaum, 2011). This discussion helps us to formulate our research focus.

\subsubsection{Ubiquitous Computing and Tangible Bits}

Firstly, we foresee the future design of ambient light systems from a macroscopic perspective by discussing the visions of Ubiquitous Computing (Weiser, 2007) and Tangible Bits (Ishii, 2004). Both of these two paradigms are proposed alternatives to conventional desk computing. Instead, they attempt to integrate computing into the physical world so that people can benefit from the advantages of computer use anytime and anywhere.

Ubiquitous computing means to push the computer into the background of our environment and attempts to make it invisible. The human-computer interaction approach introduced in the Tangible Bits vision is shifting the design of ubiquitous computing from creating different forms of GUI-supported smart devices (like smartphones, smart tablets, and smart watches) to programming the real physical world by coupling digital information to our everyday physical objects and environments. Computer augmented physical objects, for example, Tag Bowl (S. Offermans, 2016) enable the user to activate his/her preference of light atmosphere by putting his/her smart key chain into it. Also, the Shape-shifting Lamp (Yao et al., 2013) can curl from a straight strip to a rounded bulb shape and light up when people pull it.

By showing these examples, we see potential in the designing of lighting systems that are more environmentally integrated, and by involving the computational capabilities from the physical media of light sources. Our particular interest is to explore the challenges and possibilities in designing such lighting systems for information communication.

\subsubsection{Calm Technology and Ambient Displays}

Taking lessons from the "information overload" as we have mentioned in the previous section, we follow the principle of Calm Technology (Weiser \& Brown, 1995) specifically through Ambient Displays (Yarin et al., 2007).

Calm technology provides us with a general design principle to adhere: "the user's attention to the technology must reside mainly in the periphery." It has two means: the information 
presentation of ambient light can easily shift from the centre to the periphery of its user's attention, and much of the information conveyed by ambient light is present in the user's periphery rather than in his/her centre of attention.
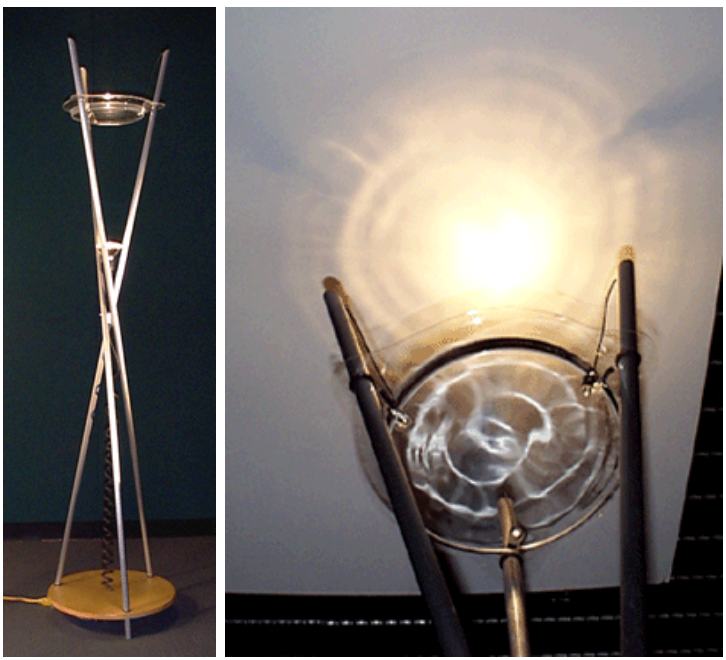

Figure 1.1. Water Lamp. (image source: http://alumni.media.mit.edu/ andyd/folio/af.html)

In addition to this, Ambient Displays show a specific approach that portrays non-critical information by means of Ambient Fixtures (Dahley et al., 2003). As one of the earliest examples, the Water Lamp (see Figure 1.1) information with dynamic wave-like light patterns that are projected on the ceiling. These light patterns are produced by shining a beam of light upward through a pan of water actuated by changing information. This Ambient Fixture presents a similar way to that what we are expecting for the future ambient light systems, as mentioned in the last section. However, we are not sure whether the need for such Ambient Fixtures in our environment is clear and strong enough. In other words, what possible extra values would these bring besides information portraying?

\subsubsection{Information Decoration and Kinetic Light Sculptures}

Information Decoration (Eggen \& Van Mensvoort, 2009) might provide a reasonable answer to the questions mentioned above. The primary goal of Information Decoration is not information itself, but the aesthetics of presenting information. It aims to provide information that we wish to be available at all times but presented in a way it can be ignored easily. Furthermore, Information Decoration points designers to the world of decoration. It inspired and directed us to investigate specific areas of the world of art that specifically relate to light 
art and kinetic art, to seek possibilities of designing ambient light systems for Information Decoration.

In the area of art, the combination of light art and kinetic art is usually called a kinetic light sculpture (Möller, 2004), which is a type of light installation in three-dimensions. It can be implemented by either manipulating physical sculptures (see Figure 1.2 (left)) to produce lights or patterns (like shadows, reflections or projections), or by manipulating lights (see Figure 1.2 (right)) to produce virtual "sculptures." In addition, recently built kinetic light/lighting sculptures have incorporated interaction techniques that enable manipulations from either its lights or its sculptures, thus adapting to the environmental changes.
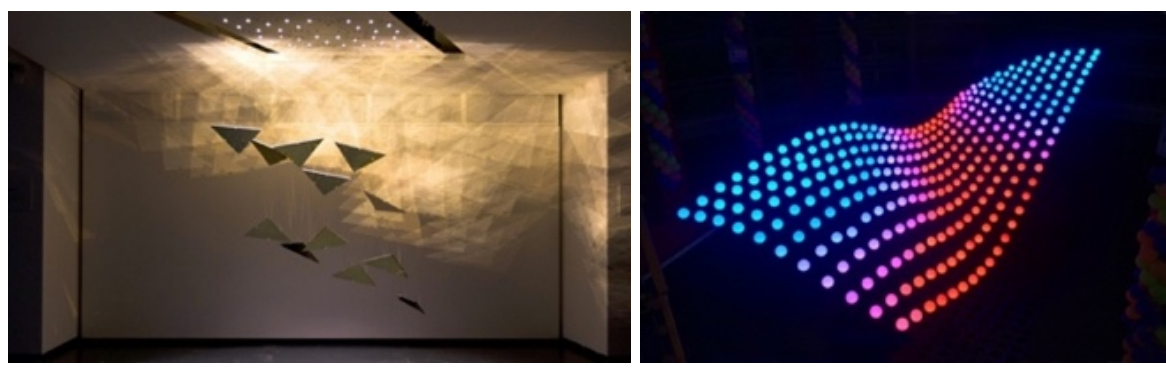

Figure 1.2. Examples of Kinetic Light Sculpture:

(Left) Tri (Appendix I, No.33), created by ART+COM Studios (video source:

https://artcom.de/project/symphonie-cinetique/); (Right) Electric Sky (Appendix I, No.05), created by KINETIC LIGHTS (video source: https://www.kinetic-lights.com/portfolio/electric-sky-2/).

The examples presented above show combinations of manipulations of light and physical media for aesthetic creation purposes. We see great opportunities of employing such art forms for designing new ambient light systems with decorative meaningful light patterns. This approach will provide designers with more freedom to play with the aesthetic quality rather than to focus on the informative quality of light only. By doing so, even if the user does not get the abstract information from the ambient displays, it is still an aesthetically pleasing decoration in his/her background without overburdening him/her (Eggen \& Van Mensvoort, 2009).

\subsubsection{Shape-changing techniques}

According to Rasmussen's identification of shape-changing techniques (Rasmussen, Pedersen, Petersen, \& Hornbæk, 2012), there are different types of change in shape such as orientation, form, volume, spatiality, permeability, etc. We have seen many kinetic light sculptures that combine light with these types of shape change. For example, the metal blades of Resonance (Appendix I, No. 23) change their orientations to create dynamic wave-like light projections, 
Lungplant (Appendix I, No. 15) changes the volume of its textile cover to sync with the breathing light, Drones Light Show (Appendix I, No. 04) change the spatial distributions of a group of Led-equipped drones to create light patterns in the air, and Shade (Appendix I, No. 24) presents a new kind of window that can block the sunlight in different patterns by changing its permeability.

Nowadays shape-changing techniques are receiving increased attention in the field of HCI. Particularly, shape-changing techniques have been explored to develop new forms of TUI (Tangible User Interfaces) (Ishii \& Ullmer, 2012), OUI (Organic User Interfaces) (Vertegaal, 2007), or Physicalization (Physical visualization) (Jansen et al., 2015). In these interfaces designs, shape-changing techniques are applied for either information displays as output or for tangible controls as input.

Meanwhile, the technologies that support shape-changing techniques have been explored. Basically, the mechanisms (Taher, Vidler, \& Alexander, 2017) for shape deformation include electromechanical actuation (Iwata, Yano, Nakaizumi, \& Kawamura, 2005) (Alexander, Weichel, Hardy, Vidler, \& Taher, 2015), pneumatic actuation (Yao et al., 2013) (Goulthorpe, Burry, \& Dunlop, 2001), hydraulic actuation (Olwal, Cheng, Follmer, Ishii, \& Leithinger, 2012), smart materials (Nakatani, Kajimoto, Sekiguchi, Kawakami, \& Tachi, 2003) (Nojima, Ooide, \& Kawaguchi, 2013), electromagnetic actuation (Frisken-Gibson, Bach-Y-Rita, Tompkins, \& Webster, 1987), and piezoelectric actuation (Kyung et al., 2011). There are also technologies that dynamically control a material's transparency (Cha et al., 2016), which can be used to create the illusion of shape-changing.

For these reasons, we see two obvious benefits to specifically apply shape-changing techniques in designing informative kinetic light sculptures: Firstly, they provide multi-modal displays of light and shape that can be organised in different levels of awareness; Secondly, they support tangible manipulation of light and information in a natural and intuitive way.

\subsubsection{Research focus}

Based on the overview and observations presented in the previous sections, we narrowed down our research scope to a more specific research focus: exploring the design space of ambient light systems that combine shape-changing with light for Information Decoration. In this way, we encourage design practitioners to take full advantage of physicality by combining the natural qualities of light and materials to leverage better ambient light systems which are aesthetically pleasing and environmentally appropriate. We temporarily define such ambient light systems as Shape-changing Lighting Systems. 


\subsection{Related Work}

To have an overall understanding of the current developments in understanding and designing Shape-changing Lighting Systems, we discuss two types of related works in this section. More specifically, we focus on studies that investigate how shape-changing and light are combined. We recognise two directions: first related work that explores augmentation of shape-changing displays with light, and second related work studies manipulatable shapechanging objects to produce meaningful light patterns. These related works include both research prototypes and art installations. Based on this discussion, we formulate the goal of our research at the end of this section.

\subsubsection{Augmenting shape-changing with light}

First, we see that related works of Shape-changing Lighting Systems in research discuss how to augment shape-changing displays with light. In this way, physical shape-changing encoding complements with light encoding through the use of RGB LEDs (Taher et al., 2015) (Gooch, 2011) or projection mapping (Iwata et al., 2005) (Tang, Sekikawa, Leithinger, Follmer, \& Ishii, 2014) (Leithinger, 2010). For example, EMERGE (Taher et al., 2015) using actuated shapechanging physical rods and RGB LEDs to display international export data, and Tangible CityScape (Tang et al., 2014) consists of an actuated shape display and a projected digital shadow display to convert abstract information into tangible forms and cross-refers tangible information of multiple city systems. These augmented shape-changing displays are seen as the next step in the evolution of a pixel by means of RGBH (Poupyrev, Nashida, \& Okabe, 2007), which is a low-resolution 2.5D display in space by moving the $\mathrm{H}$ (height) of the "pixels."
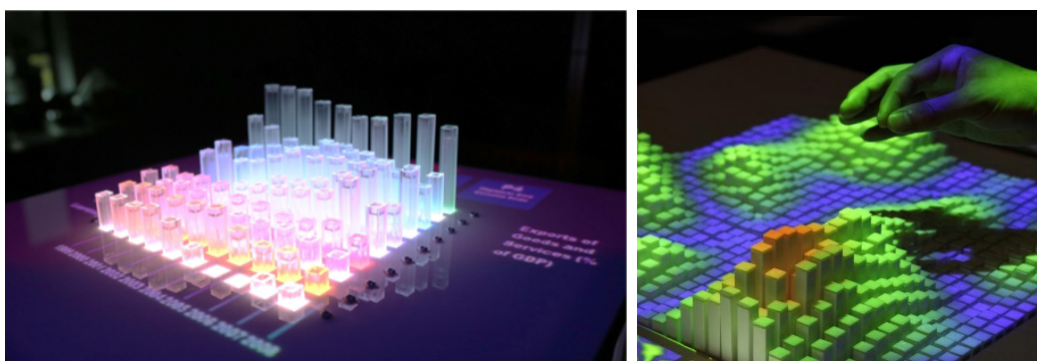

Figure 1.3. Examples of augmenting shape-changing with light in the area of research: (Left) EMERGE; (Right) Tangible CityScape (image source: http://tangible.media.mit.edu/project/tangiblecityscape)

In the area of art however, augmented shape-changing with light is regarded different from these 2.5D RGBH pixels mentioned above. Instead, artists create 3D RGBS more richly and aesthetically. The "S" refers to spatiality that is not only limited to "H." For example, NEBULA 
(Appendix I, No. 19) changes the positions of its light bulbs by rotating them in different circles to produce spiralling patterns (see Figure 1.4 (middle)). In the Lucy Light Forest (Appendix I, No. 13), more than 10,000 solar powered LED lights are used to create a luminous rolling sea of wheat (see Figure 1.4 (left)). Moreover, the KANGAROO LIGHT (Appendix I, No. 09) provides more freedom for people to manipulate the spatiality of lights (see Figure 1.4 (right)).
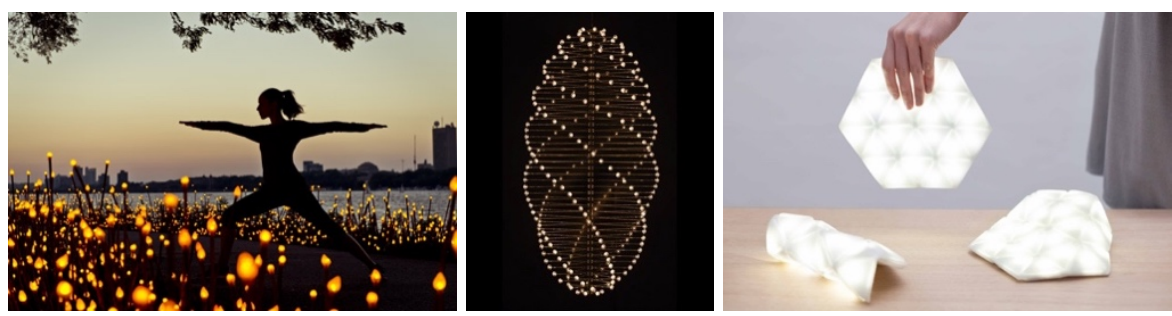

Figure 1.4. Examples of augmenting shape-changing with light in the area of art: (Left) Lucy Light Forest (video source: https://vimeo.com/207381612; (Middle) NEBULA (video source: https://vimeo.com/169616005); (Right) KANGAROO LIGHT (video source: https://vimeo.com/95299083)

\subsubsection{Sculpting light with shape-changing}

Second, the other way of designing Shape-changing Lighting Systems is using programmable shape-changing objects to produce meaningful light patterns, such as shadows, reflections, and projections. In the research area, related works are emerging persistently. These works are designed to subtly integrate with the physical architecture, enhancing, but not distracting from it. One of the earliest examples in the research area is the Water Lamp (Dahley et al., 2003) (see Figure 1.1), which has already been introduced in the previous section. Besides that, Fischer (Hornecker et al., 2015) developed a series of deformable reflectors to produce meaningful sun reflections for information depiction (see Figure 1.5 (left)). Moreover, Gleamy (Cha et al., 2016) supports ambient information display by shade patterns by manipulating the transparency of its lamp shade (see Figure 1.5 (right)).
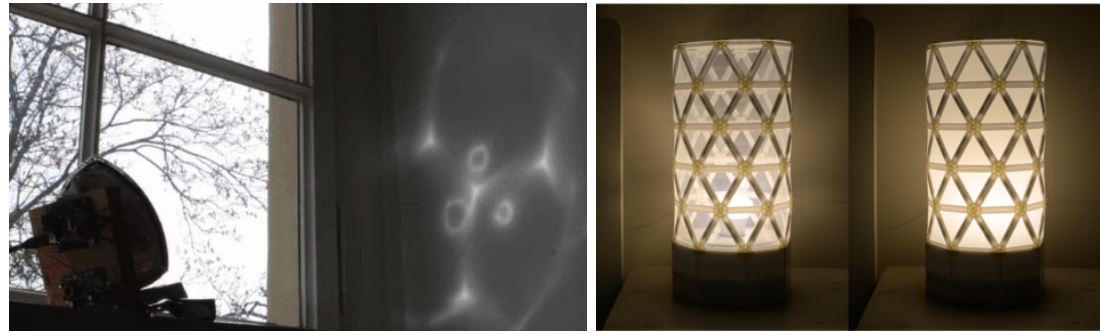

Figure 1.5. Examples of sculpting light with shape-changing in the area of research: 
(Left) Stamp Pixel 3-Matrix, one of the deformable reflectors created by Fischer; (Right) Gleamy (image source: http://cidr.kaist.ac.kr/blog/2016/02/23/gleamy/).

In the art area, the use of shape-changing objects to produce decorative light patterns has a long history. The very first art pieces we see are the Light-Space Modulator (Review \& 1988, 1988), built in 1922-30 by László Moholy-Nagy. In recent times, ART+COM Studios have explored the potential of combining kinetics with light and reflection for spatial communication and as an artistic medium. For example, Resonance (Appendix I, No. 23) consists of several reflective wave-shaped metal blades, which are actuated to generate dynamic light patterns projected on the wall (see Figure 1.6 (left)). Laurent Fort has developed many art installations by actuating different types of materials to reflect light and produce spectacular light patterns. For example, Eternal Sunset (Appendix I, No. 06) produces sunsetlike light patterns by using a floating dichroic filter in the vibrating water to reflect a beam of light from an LED (see Figure 1.6 (middle)). Paul Myoda has presented different forms of glittering machines which produce dynamic patterns of light and shadow. For example, KINETICS (Appendix I, No. 22) utilises mechanised sculptures to produce vibrating light patterns that are projected on a wall (see Figure 1.6 (right)).
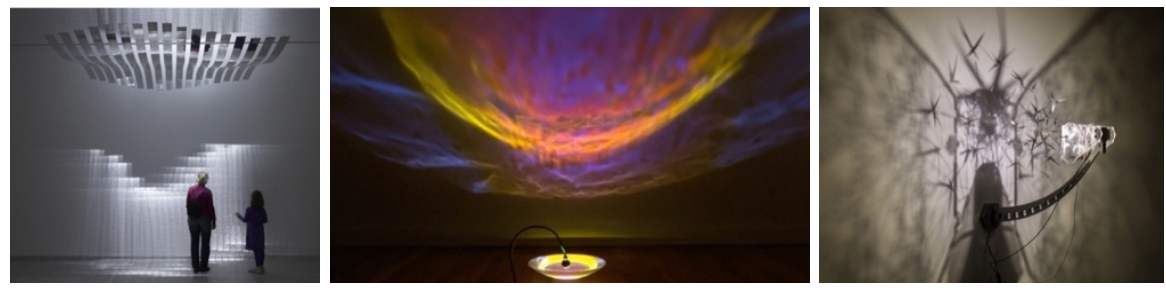

Figure 1.6. Examples of sculpting light with shape-changing in the area of art:

(Left) Resonance, created by ART+COM Studios (video source: https://vimeo.com/67561030); (Middle) Eternal Sunset, created by Laurent Fort (video source: https://vimeo.com/191648808); (Right) KINETICS, created by Paul Myoda (video source: https://vimeo.com/82157700)

We do see that some related works are research driven, but are presented with art forms. The MIT Mediated Matter group (Franchin et al., 2016) is developing a novel manufacturing platform to print optically transparent glass. They have recently presented an installation (see Figure 1.7 (left)) based on this platform for the Milan Design Week. Another example is Flow (Appendix I, No. 07), which is a conceptual lighting project developed by Selway during his internship at Phillips Design in Eindhoven. This concept presents a scenario that combines a shape-changing origami shade with light to represent the natural sky, weather, and light patterns abstractly (see Figure 1.7 (right)). 

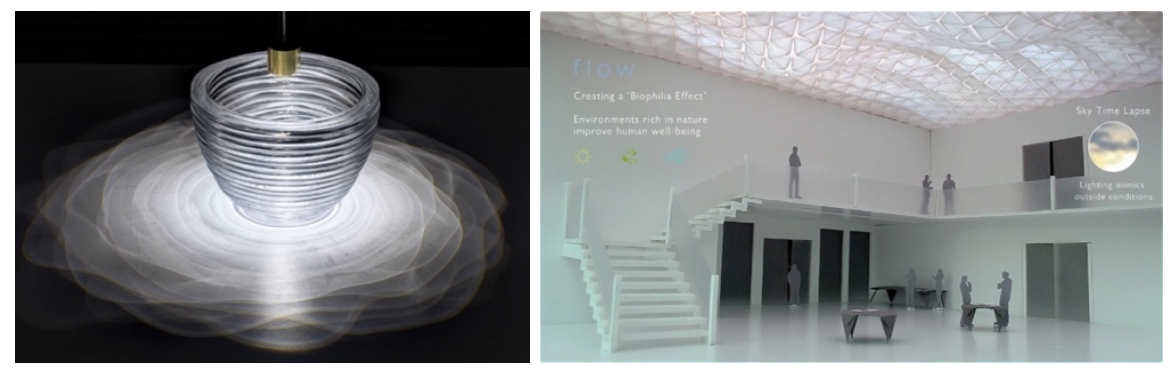

Figure 1.7. (Left) GLASS I, created by MIT Mediated Matter group (image source: http://matter.media.mit.edu/environments/details/glassi ); (Right) A concept scenario of Flow, designed by Selway (video source: https://vimeo.com/144424152)

\subsubsection{Discussion}

The related work above shows that combining shape-changing with light for lighting design has emerged for years in the areas of either research or art. Especially, the augmenting shapechanging with light has frequently be seen in the HCI field. However, in the end these are still "pixelated lights." Our research aims to reduce the overuse of "pixelated lights." We are more interested in "sculpting light by means of shape-changing", and we define this new class as Sculpting Light Systems (SLS).

The name of SLS is inspired by light art called Light Sculptures, which is a form of sculpting that utilizes light sources, such as light bulbs, the sun, and laser beams, as the primary medium of expression. Since long, artists have created installations which show different approaches to create impressive light sculptures by deploying and manipulating light sources and their physical surroundings synchronously. These works are re-identified as "sculptural lighting", "light sculpting installation", "kinetic lights", "kinetic light sculptures", "sculpting with light", "sculpting with light and shadows", and so on.

According to the principles of Calm Technology, Ambient Displays, and Information Decoration, we describe SLS along the following characteristics:

- SLS are a form of kinetic light sculptures that combine the manipulation of shapechanging objects and light.

- SLS convey information in a decorative and subtle way.

- SLS provide multi-modal displays with direct light, light patterns and shape changes of the physical components of the lighting systems.

- These multi-modal displays are calmly positioned in the periphery of the user's attention and can move to the centre of the user's attention when necessary without overburdening. 


\subsubsection{Research goal}

We believe that Sculpting Light Systems could facilitate elegant and intelligent ambient light systems in the future. However, so far, the design space of SLS has not been systematically investigated yet. To fill this gap, our research aims to generate new design-relevant knowledge that can aid the design of current and future ambient light systems that combine light with shape-changing technologies.

\subsection{Research Questions and Approach}

To achieve our research goal, we define three concrete research questions and a research approach that form the basis for this thesis. The research questions are raised from our first formal design explorations of SLS as well as from an analysis of exiting state-of the art installations. The research questions aim to understand and define Sculpting Light Systems, to provide systematic insight and overview in how to design SLS and finally to suggest systematic methods to support the design of SLS.

The related work we introduced in the last section has demonstrated diverse ways to combine shape-changing with light. However, technically speaking, few of them aim to be used as Information Decoration. So, at the very beginning of this project, we started with a practical hands-on design activity to explore the design space of SLS. By this initial design exploration, we mainly investigated the design challenges of SLS and reflected our research interests. The results lead us to a specific research direction in which we attempt to provide designers with a systematic approach rather than with anecdotical inspirations from designing SLS.

Firstly, we aim to better define and understand the design space of SLS. Can we provide a systematic overview of the design alternatives for SLS, so that a designer can benefit from it when designing lighting systems that combine light with shape-changing?

Based on a thorough analysis of relevant state-of-the-art examples of research prototypes and art installations, we intent to present an overview of the design alternatives for different SLS building blocks, in particular different components, attributes, light patterns and design styles. After that, we carried out a second iteration of design explorations to reflect on the completeness of SLS building blocks. As a result, we formulated a complete SLS Framework, which integrates all the building blocks (identified in the analysis and the design explorations) into a systematic representation of the design space of SLS. The abstract representation of all the possible SLS building blocks and the holistic point of view of the Framework will help designers in developing SLS to keep the overview, to generate and compare alternatives in an 
early design stage, and possibly avoid diving into implementation details based on preliminary ideas or inspirational examples too early.

Understanding of the design space of SLS will lead to numerous opportunities in implementation. To seamlessly integrate SLS into the context, it is important to support designers in understanding the consequences of choices for design implementations. Understanding of SLS alternatives needs to be related to understanding of the context, in particular the intended users, the environment and the specific information that will be communicated. Therefore we aim to formulate a systematic overview of interaction opportunities for SLS in the context of Information Decoration.

The second research question is how we can capture and communicate insights in the opportunities of designing with and for SLS? Can we propose an interaction model that integrates the design knowledge of understanding SLS in existing design processes?

The understanding of the SLS building blocks (components, attributes, light patterns and design styles) will provide many alternatives for the design of SLS. Based on existing interaction models from communication science and information visualization, an adequate interaction model for design SLS can be constructed. The Interaction Model will provide designers with a coherent picture of what is possible when developing an adaptive SLS for Information Decoration. More specifically, designers should be able to use the Interaction Model as a method to generate, to discover, and to compare design concepts in order to come to well-founded design decisions.

Our third research question relates to how this new design knowledge can be made operational in realistic design processes. How can the Framework on SLS and the Interaction Model on SLS be applied in developing SLS concepts?

In order to achieve this goal, we synthesize a first set of design guidelines, by which designers can generate plausible SLS concepts in a systematic way. More specifically, we formulate the design guidelines by investigating what design activities are involved in the ideation phase of developing SLS concepts and how the Framework and the Interaction Model can be deployed.

Our research aims to generate a systematic methodology for designers to aid them in the development of SLS. While SLS is in its infancy, we can only understand it from limited related work. As we have discussed in the previous section, there are many related works combining shape-changing with light, but only a few of them are designed for Information Decoration. So, we mainly adopt two types of research approaches to explore the design space of SLS: the one is a research-through-design approach (Zimmerman, Forlizzi, \& Evenson, 2007), and the other is a literature review approach. 
The research-through-design approach plays a core role throughout this project. On the one hand, we use design as a tool to explore the new possibilities of SLS. On the other hand, we evaluate the knowledge gained from literature by applying it in design practice.

In addition, we follow a literature review approach to exploit the design space of SLS. In total, we conduct two rounds of literature review. The first one aims to formulate the SLS Framework by analysing existing related examples. Those examples may not be developed for Information Decoration purposes, but help us to acquire an overview of how SLS can be structurally built. The second one aims to formulate the SLS Interaction Model by learning from other visualization reference models and available interaction techniques.

\subsection{Thesis overview}

This thesis consists of six chapters. In chapter 1 we explain the research motivation, outline our research scope, discuss related work and define the research questions and approach.

In chapter 2, we explore the design space of the ambient light systems that combine light with shape-changing by conducting concrete design activities ourselves. We've explored two design cases: Timer Lamp and Timer Shade. We also conducted user tests to evaluate how users experience our designs.

In chapter 3, we present an overview of SLS building blocks by analysing relevant existing examples. These building blocks include four basic components for construction, three types of attributes for manipulation, and three kinds of light patterns for information mapping.

In chapter 4, we demonstrate and evaluate the use of the SLS building blocks by two other designs: Reflective Clock and Time Glow. Through this evaluation, we found another valuable component for constructing SLS. After that, we integrated the total set of building blocks into one complete SLS Framework. Also, we pointed out the limitations of the Framework in design practice and our interests in the next steps.

In chapter 5, we introduce the SLS Interaction Model. This Interaction Model presents a mechanism that helps designers to describe, discover, and compare their design concepts before they put their SLS into the real world. Also, we demonstrate its usefulness by reflecting on our previous design case (Timer Lamp).

In chapter 6, we integrate our Framework and Interaction Model into the SLS Design Guidelines. The guidelines operationalise the understanding of the framework and the overview of the Interaction Model. These guidelines can help designers to generate plausible 
SLS concepts through three ideation sessions, which are collecting clues, generating ideas, and completing concepts.

In chapter 7, we evaluate the usefulness of our Framework, Interaction Model, and SLS Design Guidelines with a user study. In this study, we invited 10 design researchers in the relevant fields of SLS to use the knowledge formulated in this thesis with a hypothetical project. The results showed a general positive feedback. Also, we found out some limitations and potentials of using the knowledge formulated in this thesis in the future.

In chapter 8 , we summarise the contributions of this dissertation by reflecting on our design experience and the research questions posed in this chapter. Also, we discuss the limitations of the work presented in this dissertation, as well as the possible directions for future work. 

In the introduction we argued that designing SLS for Information Decoration is still largely an unexplored area. Therefore, in the early stages of our work, we defined the following initial research question: How can we start to explore the design space of SLS? To answer this question, we set up an explorative research-through-design process. In two design iterations, we developed and tested two SLS prototypes (Timer Lamp and Timer Shade, respectively). By reflecting on the explorative design process and its outcomes, we identified the core subjects of our further study on SLS.

In this chapter, we first explain our design goals by refining the initial research question into three sub-questions and we describe the specific context for which we developed the two design concepts. Next, we clarify the principles of designing Timer Shade and Timer Lamp. We describe various aspects of the design concepts, their implementations, and the informal user tests that were conducted for both concepts. Moreover, we reflect on the development processes for both concepts and formulate six SLS design challenges. Finally, we introduce two means to address the formulated challenges for designing SLS.

\subsection{Design Goals}

The primary goal of this early design exploration is to answer our initial question how to start the exploration of the SLS design space. More specifically, we investigate the design space of SLS in three steps:

1. Can shape-changing be integrated with lighting to provide peripheral information?

2. What are specific design challenges for designers of SLS?

3. How to face these design challenges?

To answer these three questions, we followed a research-through-design approach and created two prototypes of SLS: Timer Lamp and Timer Shade. These prototypes demonstrate general ways how to combine shape-changing with light to develop SLS.

We selected informal meetings and time management as the specific context to design for. Informal meetings are very common in the office domain. Compared to official meetings, informal meetings are more flexible in time, place, attendance, and content. These characteristics enable people to meet in a relaxed atmosphere. Timing information for such meetings seems to be the kind of data participants want to have available at all times but, at the same time, they want to be able to ignore it as much as possible. In a typical scenario, and at specific moments in time, we want to know the remaining time of the meeting, while at other moments, we do not want a clock on the wall to stress us out. To solve this dilemma, we developed two SLS that represent the progress of an informal meeting in a subtle and aesthetic 
way. On the one hand, the SLS function as a timer for users to provide an overall awareness of how much time has been elapsed or is left for the meeting. On the other hand, the SLS always feature a decorative lighting in the meeting environment that can be easily ignored without interrupting the meeting.
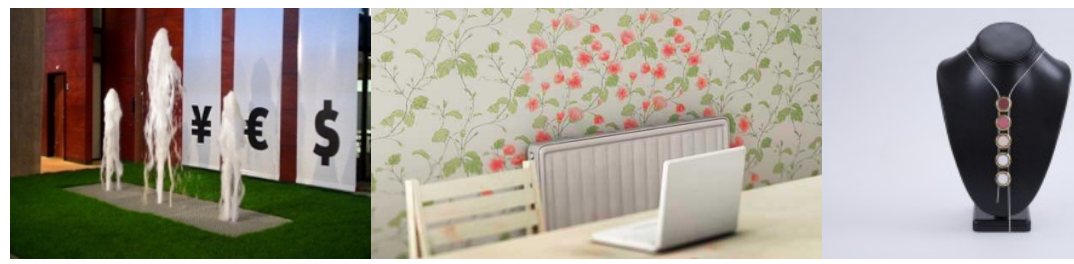

Figure 2.1. Examples of Information Decoration. (Left) Data Fountain (image source: http://www.koert.com/work/datafountain/); (Middle) Wallpaper Blooms When the Heat is On (image source: https://www.nextnature.net/2008/06/wallpaper-blooms-when-the-heat-is-on/); (Right) The Necklace (image source: http://www.marcovanbeers.nl/portfolio/the-necklace/).

The designs of Timer Lamp and Timer Shade share a typical characteristic of Information Decoration (Eggen \& Van Mensvoort, 2009) that is to consider decorative patterns in our environment as a possible information carrier. This design characteristic has been demonstrated in several earlier examples of Information Decoration (Mensvoort, 2012) such as Datafountain ${ }^{5}$ (see Figure 2.1 (left)), Wallpaper Blooms When the Heat is $\mathrm{On}^{6}$ (see Figure 2.1 (Middle)), and The Necklace ${ }^{7}$ (see Figure 2.1 (Right)). These examples transform existing decorative patterns into lively but subtle ambient displays. In our case, we mainly learned from the decorative patterns of existing lamps.

Referring to the characteristics of SLS and the selected scenarios, we set the following basic challenges to design Timer Lamp and Timer Shade:

- The system should create a decorative lighting pattern and it should be possible to dynamically manipulate its physical form and light output.

- The system should represent timing information in an aesthetic and subtle way.

\footnotetext{
${ }^{5}$ Koert van Mensvoort (2007). Datafountain. Retrieved on 29.06.2019 from https://www.mensvoort.com/home/datafountain/

${ }^{6}$ Shiyuan (2008). Wallpaper Blooms When the Heat is On. Retrieved on 29.06.2019 from https://www.nextnature.net/2008/06/wallpaper-blooms-when-the-heat-is-on/

${ }^{7}$ Marco van Beers (2012). The Necklace. Retrieved on 29.06.2019 from http://www. marcovanbeers.nl/portfolio/the-necklacel
} 
- The system should be positioned in the periphery of the user's attention and the information encoded in the lighting patterns should be able to move to the centre of the user's attention when necessary without overburdening.

- The system should support users in setting the required time available for the meeting in an easy and intuitive way.

\subsection{First Design Cycle: Timer Lamp}

Timer Lamp is our first prototype of a SLS. It is developed based on the existing light pattern of DUDERÖ (see Figure 2.2), which is a modern decorative lamp produced by IKEA. For Timer Lamp, we addressed the basic design challenges for SLS by augmenting shape-changing with light.

\subsubsection{Design concept}
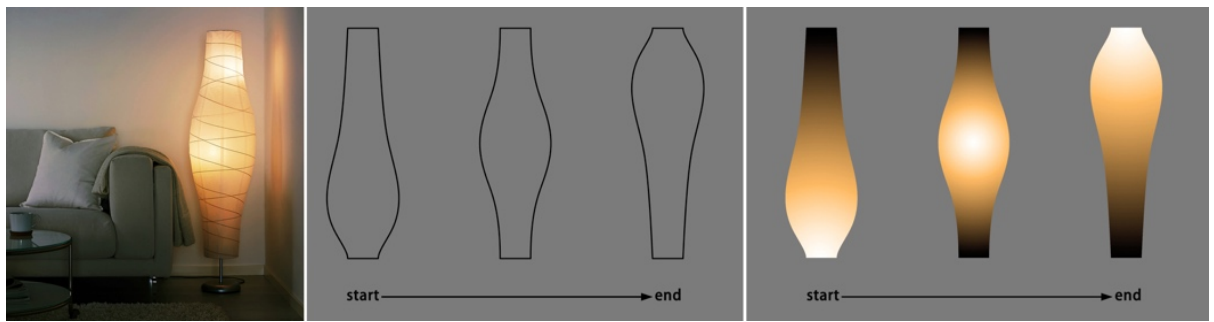

Figure 2.2. (Left) The design inspiration of Timer Lamp: DUDERÖ, IKEA (image source: http://www.ikea.com/PIAimages/0468270_PE611340_S3.JPG); (Middle) Sketching the shape-changing of Timer Lamp; (Right) Augmenting the shape-changing with light.

Figure 2.2 depicts the process of how we generate the design concept of Timer Lamp. First, we imagined Timer Lamp as a deformable DUDERÖ, in which the ridge part can float up and down (see Figure 2.2 (middle)). This shape change symbolises the pun of "time is up." Next, we considered how we could manipulate light to augment that physical shape-changing. An intuitive idea is moving the inside light sources along with the floating ridge part (see Figure 2.2 (right)). By doing so, the ridge part will be visually emphasised. That could make users more comfortable to gain timing information by recognizing the relevant position of the brighter ridge in the vertical dimension of the lamp.

\subsubsection{Design implementation}



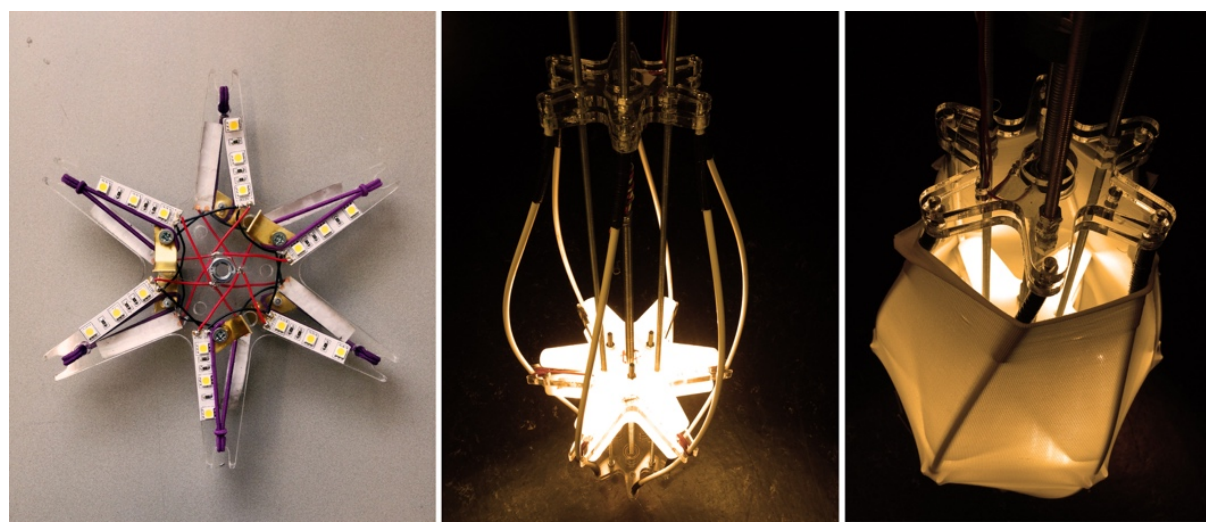

Figure 2.3. (Left) LEDs equipped pad; (Middle) Flexible framework; (Right) Elastic fabric lampshade.

The realised concept of Timer Lamp is shown in Figure 2.4. The lamp body is like an organic tube in which the light source can inflate its skin. It consists a flexible framework (see Figure 2.3 (middle)), a fabric lampshade (see Figure 2.3 (right)), and a LED-equipped pad (see Figure 2.3 (left)). A DC motor actuates the movement of the LED-equipped pad, according to which the flexible framework and the fabric lampshade change their shapes.
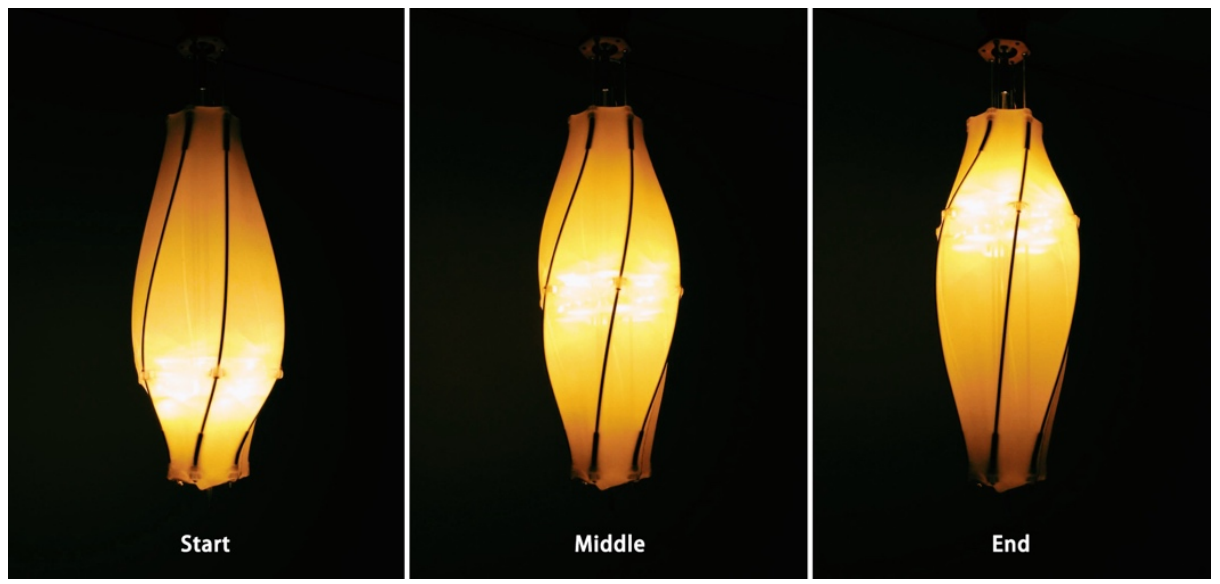

Figure 2.4. The information representation of Timer Lamp in a meeting.

We hung Timer Lamp on the ceiling in the so-called "Breakout 404" room, a room where staff members and students of the department of Industrial Design often have informal meetings (see Figure 2.5 (left)). The location of Timer Lamp is close to the area where users of the room often sit. So, the user can see Timer Lamp when he/she looks up a little bit. During the meeting, Timer Lamp presents a slow bottom-up deformation as we show in Figure 2.4. The speed of the deformation depends on the duration of the meeting that is set by the user with an Android 
App (see Figure 2.5 (right)). We considered this GUI-based interaction easy and intuitive for users to control, as Graphic User Interfaces have become a common form of interface nowadays. For example, Phillips Hue enables the user to control the light via smart devices. Once the meeting is over, Timer Lamp will immediately revert to its initial state through a quick up-bottom deformation.
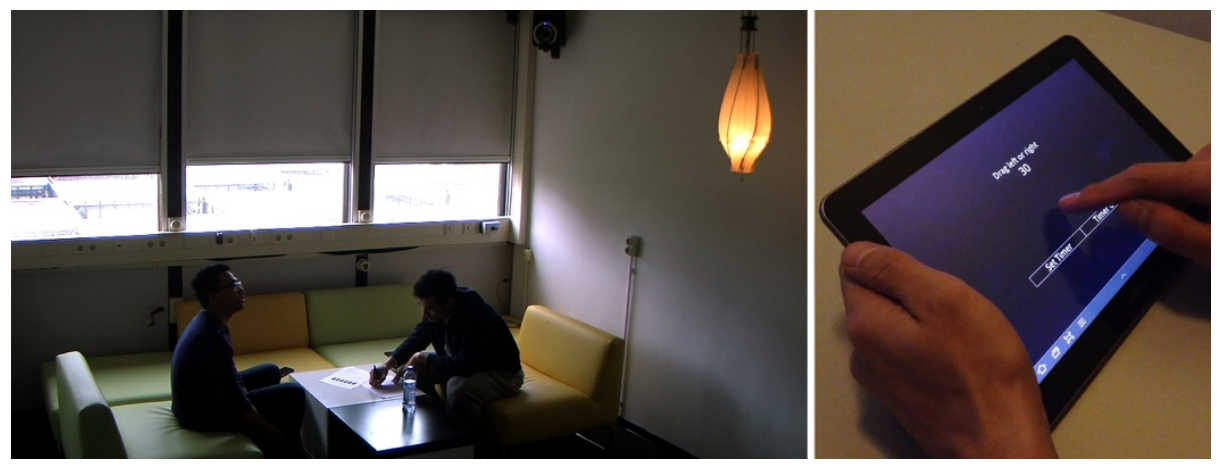

Figure 2.5. (Left) Scenario of Timer Lamp; (Right) Interaction of setting time by Timer Lamp.

\subsubsection{User feedback}

To get feedback from the users, we invited five staff members and students from the department to join an informal user test. Each of the five subjects joined a 10-minute informal meeting in "Breakout 404" (see Figure 2.4) with the experimenter, i.e. the author of this thesis. During this face-to-face meeting, a discussion was carried out about five prepared pictures (obscure advertising posters, of which logos had been blurred). By doing so, we closely observed how users experience Timer Lamp.

Before the meeting started, the subject was invited to see an introduction video ${ }^{8}$ which shows how Timer Lamp displays information by shape-changing. Then, we let the subject set the time and start the meeting with an Android App. When the meeting was over, an interview was carried out to collect the comments on the design and the user experience of Timer Lamp. In general, our participants showed their appreciation for the beauty and novelty of Timer Lamp. However, they had different opinions with respect to the capacity of Timer Lamp to represent time information. Some participants considered that approximate information of the remaining time was helpful to pace them during the meeting. However, other participants

\footnotetext{
${ }^{8}$ Jiang Wu (2014). Timer Lamp. Retrieved on 29.06.2019 from https://vimeo.com/2744374412
} 
thought that information should be more accurate. After all, Timer Lamp was not designed to indicate time information like a clock. We asked participants to comment on how to improve the design of Timer Lamp for better user experience. Below, we summarise the collected comments:

- Most participants indicated that the location of Timer Lamp was not comfortable for them to notice, and suggested to move it closer to the visual field of their primary task.

- All participates indicated that Timer Lamp looks subtle but sounds noisy. They complained that the noise distracted them during the meeting.

- Some of the participants mentioned that the shape-changing of Timer Lamp is too subtle to recognise at a glance. Instead they perceived the information directly through the position of the light source.

- Some participants said that the environmental light was too bright to notice Timer Lamp.

- Some participants indicated that the bottom-up movement of Timer Lamp only has a weak relation with the time passing, and they suggested to use the top-down movement using the metaphor of gravity. One participant even forgot the coding of our ambient display by misinterpreting the movement of the light source of Timer Lamp as top-down.

\subsubsection{Reflection on Design challenges}

Based on reflections about either the difficulties we tried to overcome when we were developing Timer Lamp or from the inadequacies indicated by the participants in the informal user confrontation we formulate three design challenges for developing SLS.

The first design challenge for SLS is to create calm kinetic structures that not only visually change in a subtle way, but also sound quiet. The implementation of Timer Lamp was harder than we expected. On the one hand, it took much time to design a complex kinetic structure to combine the mechanical shape-changing with the moveable light fixture. On the other hand, it took much effort to reduce the noise produced by the motor and the mechanical transmissions.

The second design challenge for SLS is to consider suitable materials that can not only smoothly support the kinetic structure but also can produce aesthetically pleasing light effects. In the implementation process of Timer Lamp, it was difficult to find the appropriate fabric for the lampshade. We tried fabrics that could nicely diffuse light but that turned out to be too tense so that the DC motor could not work smoothly. Also, we tried other fabrics that could work suitably with both the flexible framework and the DC motor but that was too permeable so that the diffusion effect became ugly. 
The third design challenge for SLS is to minimise the impacts from the environment to ensure a stable ambient display. Timer Lamp was recognised as a beautiful kinetic light installation, but the performance of its information display turned out to be too fragile and too subtle in a real environment. For example, the uncontrollable daylight sometimes made Timer Lamp less visible.

\subsection{Second Design Cycle: Timer Shade}

Timer Shade is our second SLS prototype. It is also designed to support the informal meeting scenario. The design of Timer Shade is based on a typical decorative ceiling lamp which has a hemisphere form. For example, IKEA's 365+ BRASA showed on the left of Figure 2.6. In this iteration, we applied the lessons learned from the first design cycle to develop Timer Shade. We designed a more recognizable shape-changing, reduced the noise induced annoyance, and located the information display closer to the users of the meeting room. With Timer Shade, we investigated the design challenges of SLS by using shape-changing to sculpt light.

\subsubsection{Design concept}

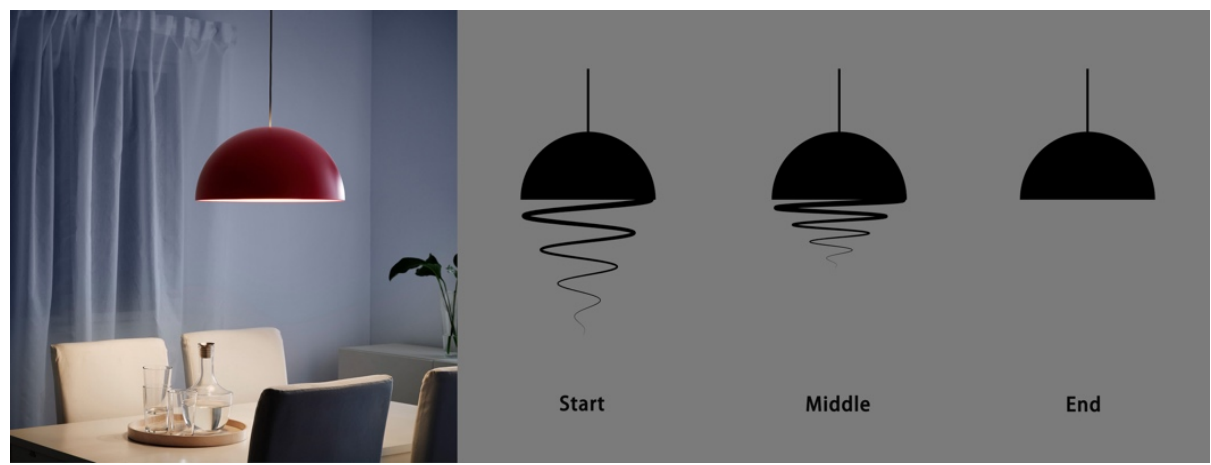

Figure 2.6. (Left) An example of the design inspiration of Timer Shade: 365+ BRASA, IKEA (image source: http://www.ikea.com/PIAimages/0472142 PE613946 S5.JPG);

(Right) The sketchy concept of Timer Shade.

The design concept of Timer Shade came from an idea of how to transform a hemispherical ceiling lamp into a deformable ceiling lamp that can produce light effects. We thought of adding a retractable cap (see Figure 2.6 (Right)) on the bottom of the lamp. This approach could be simpler than making a deformable hemispherical lamp cover. By extending the existing cap, we can imagine that not only the shape of the lamp body changes but that also the shadows projected on the table will change. For the shape change, its linear bottom-up 
movement indeed can present the progress of the meeting. For the shadow change, we were not clear about its actual appearance.

Then, we explored the imagined concept with a first test prototype (see Figure 2.7). This prototype is made of a retractable cap, a wooden bowl (used as lamp cover), and a power LED. The cap is taped on the opening of the bowl, and the power LED is positioned on the inner bottom of the bowl. By manipulating the retractable cap, we saw a significant change for the shadows cast on a white table (see Figure 2.8). More specifically, with the bottom-up movement of the cap, not only the scale of the shadows was enlarged, but also the sharpness of the shadow is reduced. This fade-out effect is a good metaphor for something that is receding, but its pattern is lacking the association with time. So, for the real prototype of Timer Shade, a better pattern of the coiled cap is needed.
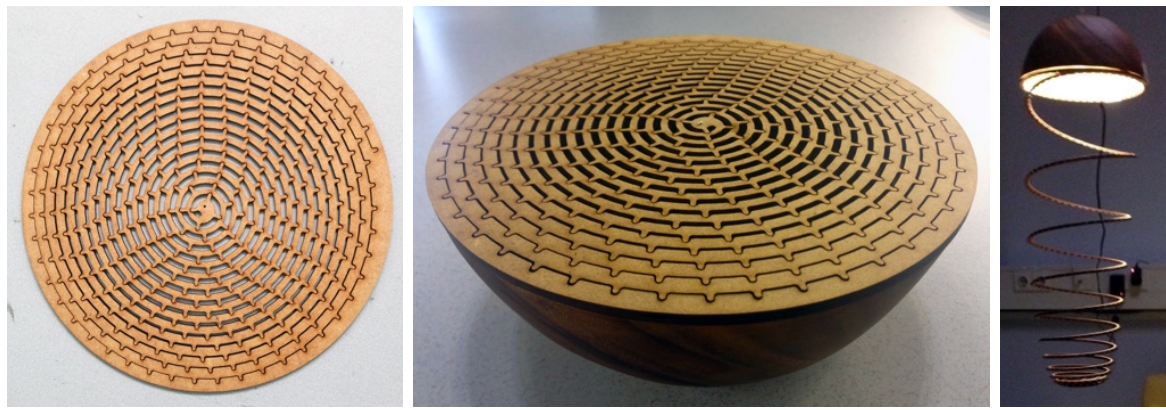

Figure 2.7. The test prototype of Timer Shade.
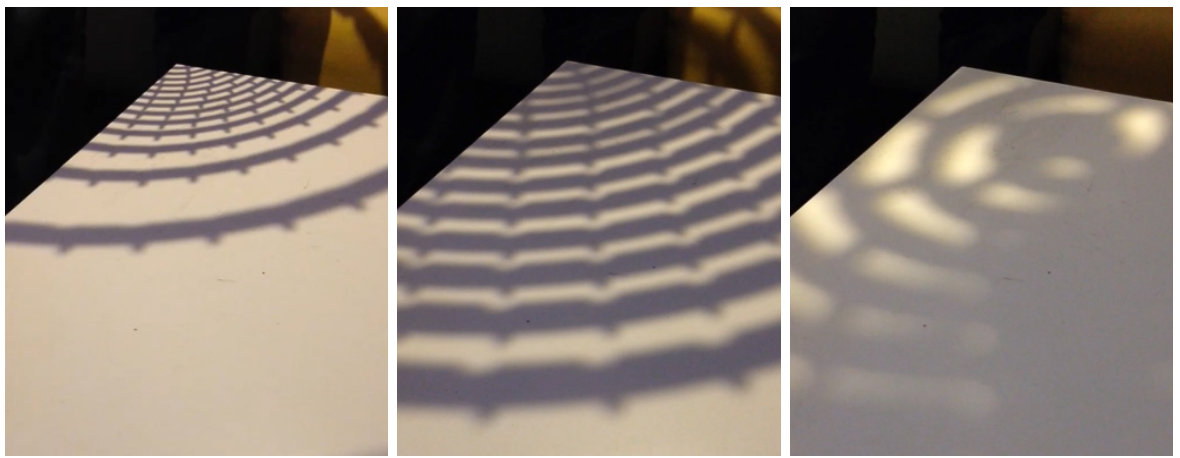

Figure 2.8. The shadow effects created by the test prototype of Timer Shade when we pulled the retractable cap up.

\subsubsection{Design implementation}


Timer Shade is implemented as a ceiling lamp located in a room within our office. This room is a closed and quiet place either for personal discussions or for a short break alone. In this room, we set up a meeting area (see Figure 2.11 (Right)) for Timer Lamp to support a face-toface meeting for two persons. Timer Shade is the main light source of that area. For the other area of the room, ten small halogen ceiling spotlights were used as auxiliary lighting to provide not only low backlight but also a warm atmosphere. We considered this environmental light to match the texture of wood we used for Timer Shade.

Based on the experiences with the test prototype, we designed a new retractable cap (see Figure 2.9 (Left)) for Timer Shade. Also, we used a high-power LED (see Figure 2.9 (Middle)) to enable Timer Shade to function as the main light source of the meeting area. The newly designed cap is formed like growth rings of a tree and is associated with the natural wood grain of the lamp cover. When turning on the light, we see a beautiful luminous effect (see Figure 2.9 (Right)).
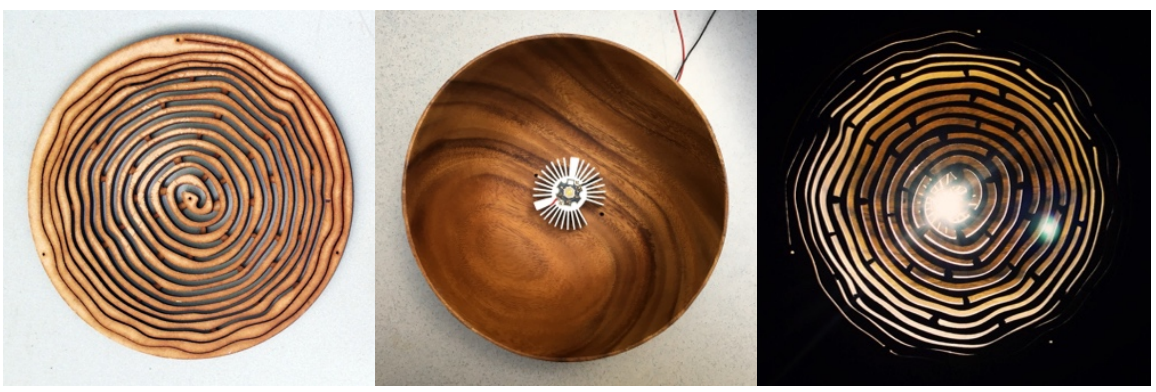

Figure 2.9. (Left) The newly designed cap for Timer Shade; (Middle) The high-power LED inside Timer Shade; (Right) The luminous effect of Timer Shade.
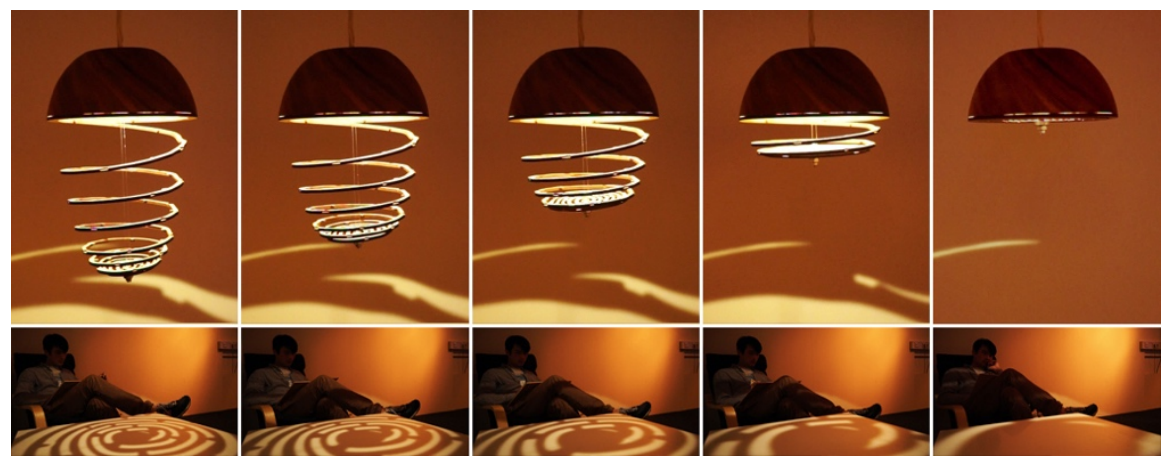

Figure 2.10. The shape change (top) and shadow change (bottom) of Timer Shade during a 20-minute meeting. 
Timer Shade presents the progress of a meeting through both shape change and shadow change (see Figure 2.10). The shape change is a uniform bottom-up movement (see Figure 2.10 (Top)) of the retractable cap. It is realised by actuating a servo motor to pull up the cap with a fishing line with a constant speed. The servo motor is located at the next door so that no noise will disturb users this time. The shadow change is a ring-by-ring fade-out light effect that projected on a white table (see Figure 2.10 (Bottom)). Besides this light effect, we also use an on/off-switching to indicate the start and end of the meeting.

Also, we designed a new interaction for users to set meeting time by physically manipulating Timer Shade, instead of having to use the Android App. More specifically, Timer Shade enables the user to set the duration of the meeting by pulling down the coiled shaped cap (see Figure 2.11 (Left)). This control provides users with a haptic feedback, i.e., they feel a "click," when they pull the retractable cap to positions corresponding to 5-minute, 10-minute, 15minute, or 20-minute meeting time, respectively. We envisioned that this interaction could help people to strengthen the impression of how time information is mapped to the shape change and the shadows change.
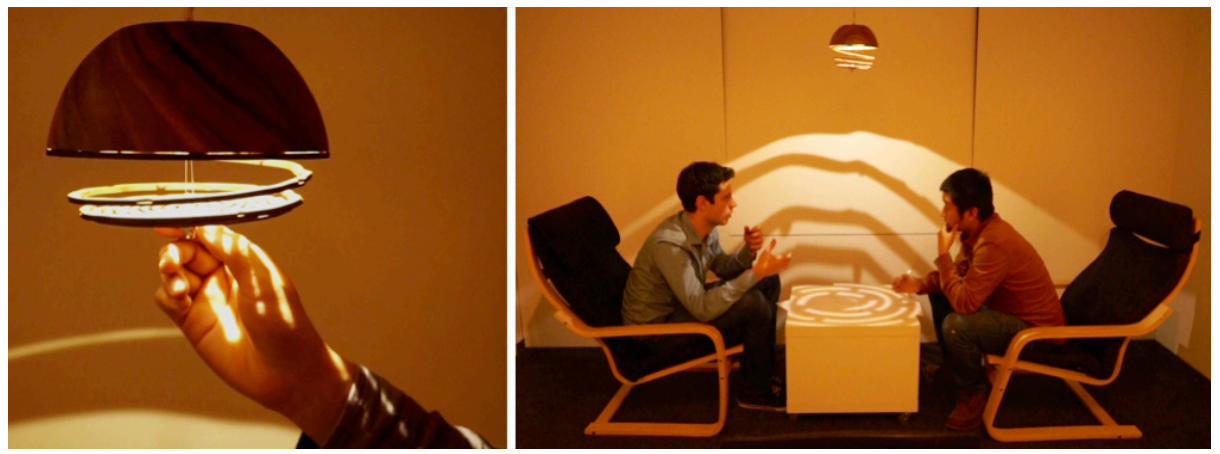

Figure 2.11. (Left) Interaction of setting time by Timer Shade; (Right) Scenario of Timer Shade.

\subsubsection{User feedback}

To get feedback from the users on the design of Timer Shade, we invited in total ten staff members and students from the department of Industrial Design to join an informal user-test. The participants were randomly divided into five pairs, and each pair was asked to attend a 20 minutes face-to-face meeting. The content of the meeting was a brainstorm session that consisted of two sessions, each of which should be finished in 10 minutes. Other devices that could be used to check time, besides Timer Shade, were not allowed during the meeting. Different from the meeting in our first iteration, none of the researchers was present at the meeting this time. Instead, we placed a video camera in the meeting room to record the whole 
meeting. That helped us to analyse how our participants behaved towards Timer Shade during the meeting.

Before the start of the meeting, the participants were invited to watch an introduction video 9 that shows how Timer Shade displays information. Next, we let the subjects set a 20-minute meeting by pulling down the retractable cap of Timer Shade. After that, we activated the motor and then the coiled shaped cap would be pulled up with a string that connected to the motor with a constant speed. When the meeting was over, we switched off the motor and turned off the light of Timer Shade. Then, we interviewed our participants to collect their comments on the design and the user experiences of Timer Shade.

From the video records, we observed that our participants intended to get time information by glancing Timer Shade. Notably, near the end of the meeting, they glanced at the lamp repeatedly, until the light was turned off. From the interviews, we found that the information display of Timer Shade was generally recognised as being "helpful" in supporting time management during an informal meeting, and also "delightful" to become aware of the passing of time in a subtle and unobtrusive way. Besides, there were a few complaints on the distractions caused by Timer Shade during the meeting. These distractions related to the strong contrast of shadow patterns on a white table while they were reading and writing. Also, the soft sound and visible tremble generated by the friction between the components caused some distractions. These participants suggested to move the shadow to another object around them, for example, the wall, or they suggested reducing the contrast of the shadow. In addition to the feedback on the information displays of Timer Shade, our participants appreciated the interaction that allowed to set the time for the meeting, and they recognised it as being "intuitive" and "easy-to-use."

\subsubsection{Reflections on Design challenges}

At the end of this design cycle, we are able to formulate three new design challenges of SLS. Again, these challenges are based on the reflections on either the difficulties we met in the design process, or the feedback from our participants in the user-confrontation sessions.

The fourth design challenge for SLS is to provide more affordable ways for designers to predict the light patterns when they are generating design concepts. Timer Shade produces elegant shadows, which were, however, hard to predict precisely in comparison with the

\footnotetext{
${ }^{9}$ Jiang Wu (2014). Timer Shade. Retrieved on 29.06.2019 from https://vimeo.com/274438399
} 
diffused light produced by Timer Lamp. That is why we decided to make an (extra) experimental prototype before implementation of the final concept. This experimental prototype helped to concrete the final concept of Timer Shade; its costs in terms of time and effort was relatively doable. But imagining a complicated SLS, costs can be significantly more expensive.

The fifth design challenge for SLS is to involve the specific environment to facilitate wellintegrated ambient displays. The design of Timer Lamp was more than just combining shapechanging with light. The qualities of its surroundings did matter as well. For example, we used a white table to present the shadows clearly. Also, we used the warm and dim backlight to create an atmosphere that can match the sense of wood. Moreover, to enable both the shape display and the shadow display of Timer Lamp to be perceived in a recognizable but unobtrusive way, we had to make a trade-off in the deployment of lamp, table and chairs. As a result, the height of the shape display was a little bit beyond people's viewing angle when they are sitting. Otherwise, if we moved Timer Shade lower, the contrast of the shadows would get stronger which might cause distractions.

The sixth design challenge for SLS is to exploit the tangibility of shape-changing to support natural interactions for user to manipulate ambient displays. Timer Shade enables the user to set time by pulling down its retractable cap. It showed that shape-changing not only can be used to display information as an output, but also can be used as an alternative input for users to manipulate data. This tangible interaction was experienced as "intuitive" and "easy-to-use" by our participants. Further, we considered that this tangible interaction possibly can help users to better understand the ambient displays of SLS.

\subsection{Means to address SLS design challenges}

Timer Lamp and Timer Shade were our first two SLS prototypes in the absence of earlier experience in designing for SLS. They were designed by animating an existing decorative lamp in order to convey information. This approach was helpful to quickly come up with early SLS concepts, but these early concepts proved to be far from ready for implementation. By following an explorative research-through-design process we uncovered six design challenges that were reflected upon in the previous sections. In this section, we consider these design challenges from the perspective of a designer.

\subsubsection{Analysis of relevant state-of-art examples of SLS}


The first means to get access to available knowledge concerns a review of state-of-art examples that relate to SLS. This review can provide designers with references of available techniques (Design challenges 1 and 2), the possible environmental impacts (Design challenge 3 ), the potential light patterns (Design challenge 4), and the environmental set-up (Design challenge 5) to combine shape-changing with light. The examples can be collected from several fields such as kinetic light sculptures, shape-changing interfaces, or installation art of light.

\subsubsection{Overview of Architecture of SLS}

Timer Lamp and Timer Shade demonstrate two general ways of combining shape-changing with light for SLS. One is using light to augment shape-changing, the other is using shapechanging to sculpt light. As we have seen in the reflections on these two designs, SLS design would be incomplete without considering manipulation of aspects of the environment (Design challenge 5) and the user (Design challenge 6). To take these aspects into account, a complete system architecture of SLS is essential for SLS designers. This architecture should provide an overall view of mechanisms or building blocks on how SLS can be constructed and manipulated to create ambient displays.

\subsection{Conclusion}

In this chapter, we have presented two early explorations of designing SLS both following a research-through-design approach. Two different SLS prototypes have been created, Timer Lamp and Timer Shade, to support time management during an informal meeting. Timer Lamp was designed by using light to augment the shape change, while Timer Shade was designed by using shape change to sculpt light. By reflecting on the design processes and on the feedback from users, we formulated six SLS design challenges. In addition, we proposed two necessary means for designers to start to address these six design challenges. We now conclude the work we presented in this chapter by reflecting on the three sub-questions that were raised at the beginning of this chapter.

Firstly, SLS can successfully be used as a communication medium for the abstract visualization of peripheral information. On the one hand, SLS has the opportunity to convey information with different modalities. We presented two examples how to do that, the shape-changing (the movement of the retractable cap of Timer Shade), and the augmented shape-changing (the deformation of the illuminated lamp body of Timer Lamp). Other examples that were shown include the initial light from the light sources (turning off the light of Timer Shade when the meeting is over) and the light patterns (the shadows produced by Timer Shade). We believe 
these information modalities potentially can be used to adapt to different levels of awareness of users. On the other hand, SLS can support tangible interactions by merging the output and input of shape-changing to provide users with a natural way to manipulate the ambient displays. For example, Timer Shade enables the user to set the duration of the meeting by pulling down the retractable cap.

Secondly, in this study we formulated six design challenges for the design of SLS by reflecting on the design processes and the outcomes of user tests. These design challenges include some practical issues on creating calm kinetic structures, looking for suitable materials, minimizing the impacts from the environment, and predicting light patterns. Also, these challenges indicate some potential issues on integration with the surroundings and on exploiting the tangibility of shape-changing.

Finally, we proposed two means, to address the six design challenges from a designer's perspective. One approach concerns a review of state-of-the-art examples that relate to SLS. This analysis will be initiated in the next chapter. The second approach concerns the development of a complete system architecture of SLS. This overview will be constructed and validated in the remaining of this thesis. 

To achieve a basic understanding of the design space for Sculpting Light Systems (SLS), especially on how to implement SLS in a modular, configurable way, we construct the building blocks of SLS in this chapter. By presenting the vocabulary of SLS, these building blocks provide practitioners with a systematic overview of the design alternatives of SLS. The development will be based on a thorough analysis of a sample of related works of SLS that combine light with shape change. In section 3.1 examples will be selected and analysed, while in section 3.2 the results of the analysis will be discussed with respect to potential application for Information Decoration in the design space of SLS. From the findings, an overview of the SLS building blocks will be constructed in section 3.3. Furthermore, four typical styles of SLS will be introduced in section 3.4. These four styles of SLS can potentially fit in five scenarios of implementing SLS for Information Decoration. At the end of this chapter in section 3.5, we will discuss the limitation of the SLS building blocks, and we will formulate conclusions on the potential of using the SLS building blocks.

\subsection{A Review of the Design Space of SLS}

In the previous chapters we have formulated a definition of Sculpting Light Systems. Sculpting Light Systems (SLS) are lighting systems (or lighting installations) that intent to convey information in an unobtrusive and aesthetically pleasing manner by actively manipulating light sources and shape-changing objects. SLS differ from other informative or artistic lighting systems by the features of aesthetically pleasing, subtly meaningful, tangibly interactive, and environmentally appropriated.

In order to explore the design space of SLS and acquire an overview of the existing research and design practices relevant to SLS, a review of state-of-the-art examples has been conducted. The works selected all feature a combination of light and shape change, and they represent either design or research projects. Some of the works were not implemented for information display purposes originally, however, they show a big potential to be used for Information Decoration and provide inspiration and insight for designing SLS regarding both implementation and user experience of SLS. Therefore, we included these works in this review.

In this review, we first analysed the common elements of SLS from four perspectives: (1) what kind of light sources and physical objects are implemented, (2) how are the light sources and physical objects manipulated, (3) what attributes are used for the manipulations, and (4) how are the light sources and physical objects deployed. Then, we discussed the potentials of using SLS for Information Decoration and give a new, more specific, definition of SLS.

\subsubsection{Sample for analysis}


We first collected related examples of SLS. In these examples, we saw only a very small number of research projects, which were collected on Google Scholar by searching the combination of the key words of "shape-changing", "light", and "information displays". The published time of these research projects are distributed from 1998 to 2016 sporadically. While, in the art and design area, we found a large number of examples in the art and design area from the Internet (Google, Vimeo, and YouTube) by searching the key words such as: "shape-changing light", "light sculptures", and "interactive lighting". To gain the latest perspective, we only reviewed the state-of-the-art design projects released between 2010 and 2016.

In total, we selected a sample of 33 instances, which meet both criteria: the system was designed by combining shape change with light; and the shape change of physical objects influences the light path and results in manipulable light patterns. These 33 examples are summarised in Appendix I. For each example, the following information is presented: title, a short description, the authors or creators, a characteristic picture, links to the original sources, and if available a link to a video demonstrating the object.

\subsubsection{Light sources \& physical objects}

Light sources and physical objects are two essential elements for implementing SLS. Light sources offer the light that will be sculpted by physical objects. The first step of our analysis was to investigate what kinds of light sources and physical objects are used in our sample. By doing so, this review provides designers with several alternatives of light sources and physical objects for the implementation of SLS.

\section{Light sources}

Light sources refer to the body where visible light comes from, including both natural and artificial sources. For natural light, quite a few examples in our sample apply sunlight and ambient natural light. For examples, Digital Sundial (Appendix I, No.28) (see Table 3.1 (first from left)) can produce the shadows of digital colour by blocking the sunlight; Shade (Appendix I, No.24) (see Table 3.1 (second-left)) blocks the ambient natural light from outside to create ever-changing patterns on a wall of smart glasses.

Artificial light sources include traditional light bulbs and intelligent light sources. The traditional bulbs provide constant light properties until people switch them off. For example, Soft Light (Appendix I, No.27) (see Table 3.1 (third from left)) has a flexible and touchable cover to create a warm and soft experience of light. The intelligent light sources basically include LEDs, OLEDs, and smart light bulbs. They are well represented in our sample. These light sources offer rich means to manipulate light properties, such as on/off, intensity, and 


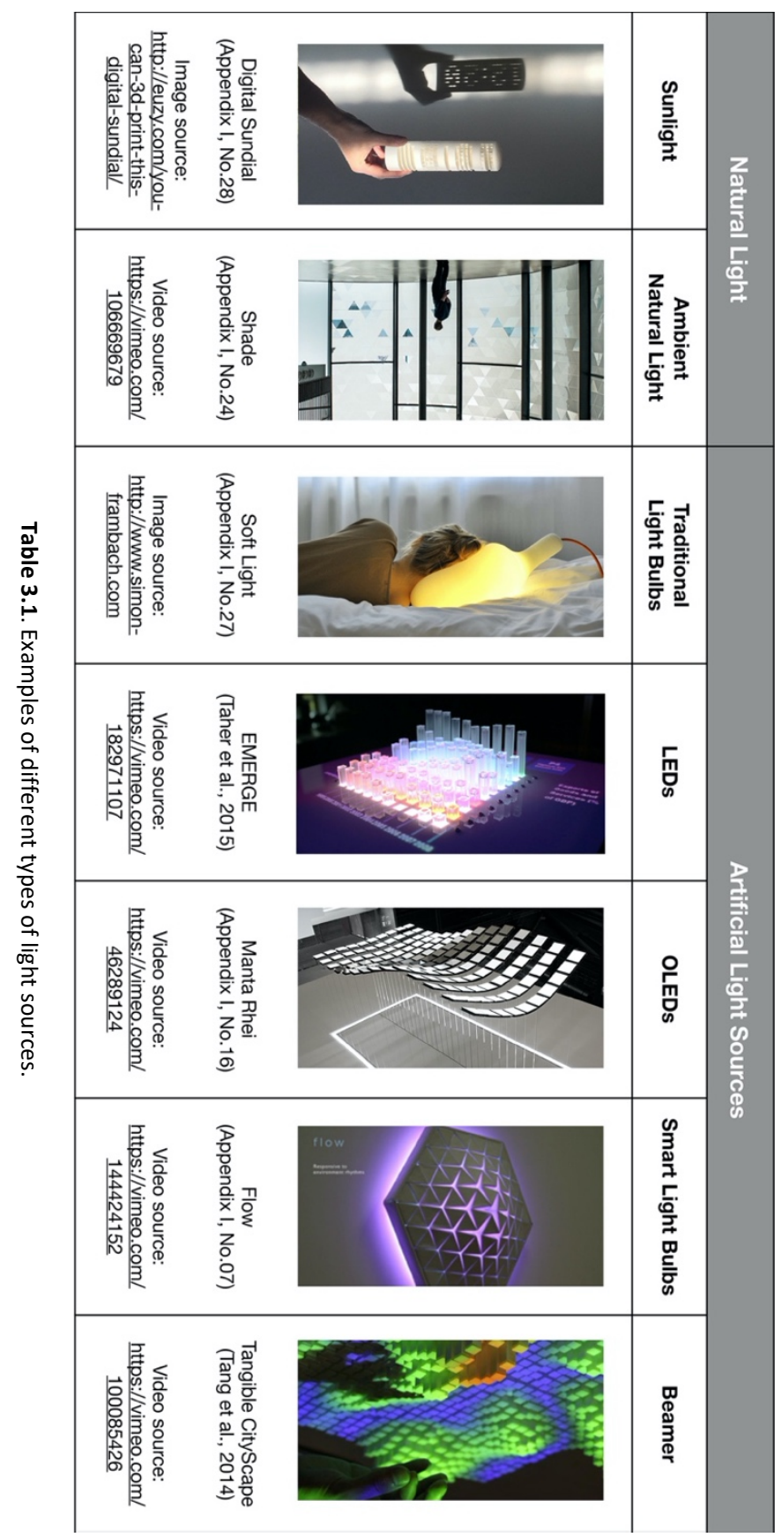


colour (temperature). For example, EMERGE (Taher et al., 2015) (see Table 3.1 (fourth from left)) uses actuated shape-changing physical rods and RGB LEDs to display international export data; Manta Rhei (Appendix I, No.16) (see Table 3.1 (fourth from right)) changes the intensity of its OLED slices with the movement of its light fixtures; Flow (Appendix I, No.07) (see Table 3.1 (third from right)) presents different colours by a Phillips Hue lamp ${ }^{10}$ inside its cover to show the environmental conditions. Additionally, we also notice that there are a few examples in our sample that utilise beamers as intelligent light sources to produce more complex light patterns. In these examples, the physical shape-changing encoding complements with the light encoding through projection mapping. For examples, Tangible CityScape (Tang et al., 2014) (see Table 3.1 (second from right)) augments the shape change of its interactive tangible interfaces with projected images.

\section{Physical objects}

In SLS, the physical objects, in particular, refer to objects that are implemented to produce light patterns by physically changing the light path. According to the stages in which the change of light path occurs, we identified three types of physical objects in our sample: the physical objects for holding light sources, the physical objects for creating light patterns, and the physical objects for presenting light patterns.

First, we distinguished physical objects that change the light path at its starting point. They are light fixtures which hold the light sources. In our sample, the shape change of this type of physical objects produces three-dimensional luminous light sculptures either by altering the initial light direction or by changing the spatial position of light sources. For example, Kinetic Wall of Lights (Appendix I, No.11) (see Table 3.2 (first from left)) creates the illusion of shape change by directly changing the orientations of its LED panels.

Second, we identified physical objects that change the light path halfway. They are used for creating light patterns by means of diffusing, blocking, reflecting or refracting the light from the light sources. In our sample, these kinds of physical objects include shades, diffusers, reflectors, and refractors. For examples, Babele (Appendix I, No.02) (see Table 3.2 (second from left)) creates a light gap by disassembling its lamp shade; KANGAROO LIGHT (Appendix I, No.09) (see Table 3.2 (third from left)) has a flexible cover, which is used to diffuse the light; Resonance (Appendix I, No.23) (see Table 3.2 (fourth from left) uses a kinetic body of mirrors to produce ever-changing light projections that cast on the wall; Water Lamp

\footnotetext{
${ }^{10}$ Koninklijke N.V (2013). Philips hue. Retrieved on 29.06.2019 from http://www.meethue.com/
} 


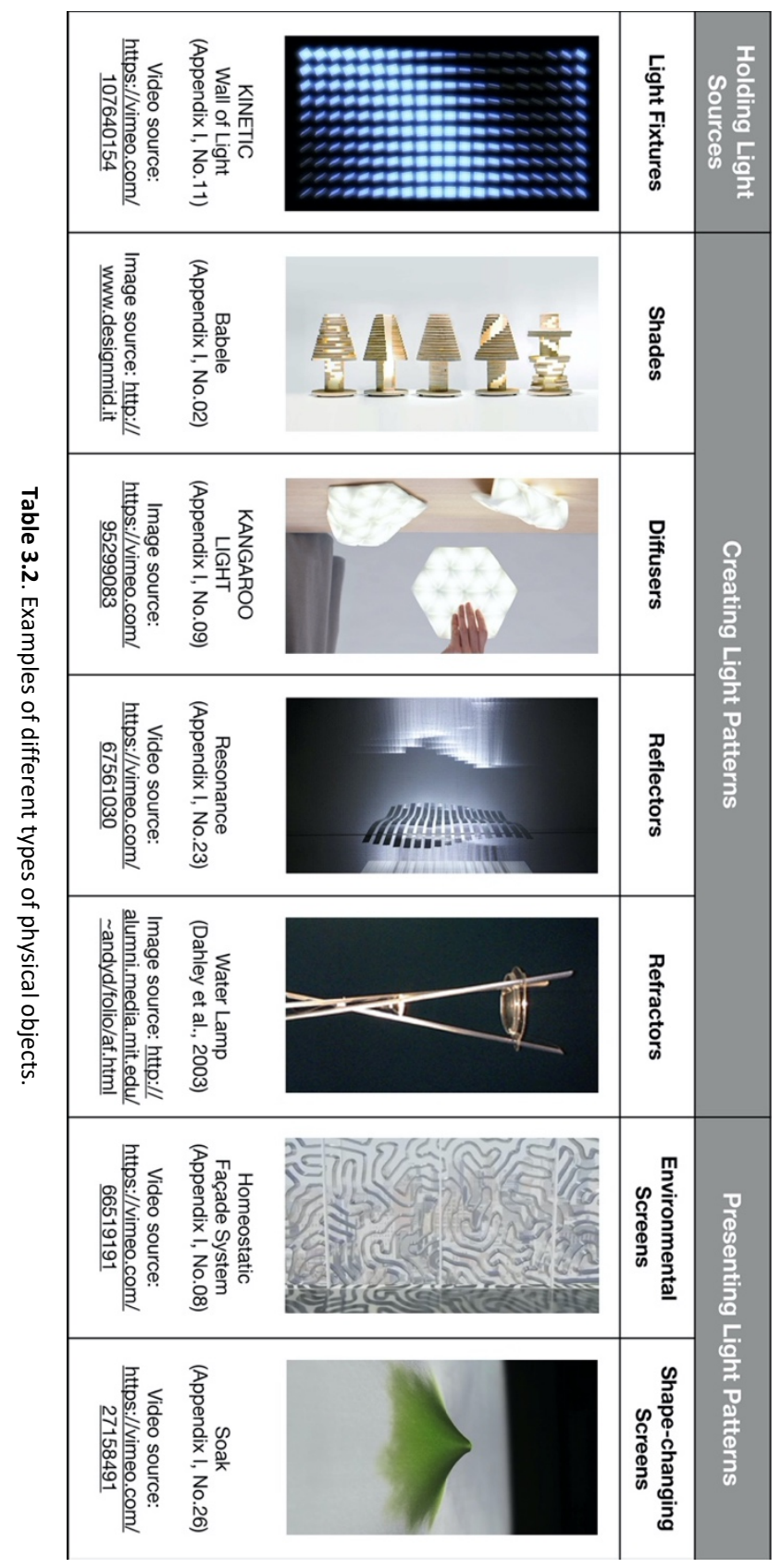


(Dahley et al., 2003) (see Table 3.2 (third from left)) uses a basin of water to produce refracted light projections that cast on the ceiling to display digital data in the user's background.

The last type of physical objects also changes the light path at the halfway but is only used to show the projected light patterns. This kind of physical objects could be the environmental screens such as: wall, floor, and ceiling. For example, the floor implemented in the case of Homeostatic Façade System (Appendix I, No.08) (see Table 3.2 (second from right)). Also, such kind of physical objects could be a shape-changing screens (interfaces). For example, Soak (Appendix I, No.26) (see Table 3.2 (first from right)) reacts the user that is touching on a fabric with animated projections.

\subsubsection{Manipulations}

In the previous section, we described different light sources and physical objects that are utilised in our sample; in this section, we will investigate how these elements are manipulated. Depending on whether the manipulation is triggered with or without a user's intentional intervention, we can characterise two means of manipulations (see Table 3.3): active manipulations and passive manipulations.

\section{Active manipulations}

Active manipulations enable the light sources or physical objects to actively change their state without the intentional intervention of a user. Such kind of manipulations might be controlled by computers or follow a certain inherent natural quality of the light sources or physical objects. For example, the flexible fixtures of Manta Rhei (Appendix I, No.16) (see Table 3.3 (bottom-middle)) automatically change their shapes to form an undulating wave; the lampshade of Responsive Lamp (Yao et al., 2015) (see Table 3.3 (bottom-left)) opens up to leak light when it is turned on caused by its material quality of thermal expansion; Shade (Appendix I, No.24) (see Table 3.3 (top-left)) applies ever-changing natural sunlight as its light source, which, of course, cannot be manipulated by user; and Flow (Appendix I, No.07) (see Table 3.3 (top-left)) employs a Phillips Hue lamp as its light sources, which respond to environmental rhythms by changing its colour and intensity.

\section{Passive manipulations}

Passive manipulations mean to change the state of light sources and physical objects under the user's intentional control. The passive manipulations of light sources can be realised using an extra independent lighting interface. For example, to turn on or off a lamp with a switch or an App on a smartphone. Also, the light effect can be the result of a tangible manipulation of 


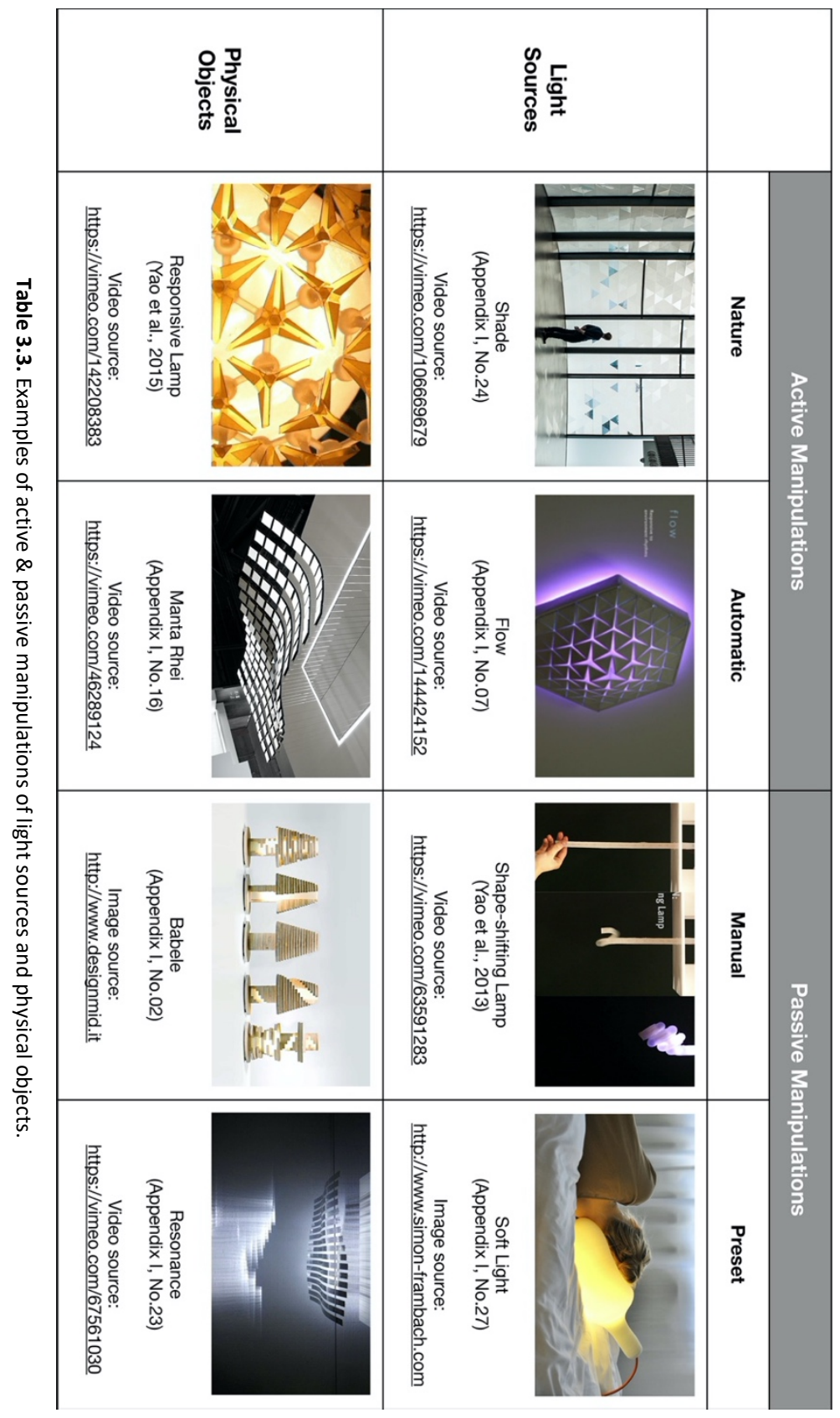


relevant physical objects. For example, turning on the light of the Shape-shifting lamp (Yao et al., 2013) (see Table 3.3 (top-middle)) by pulling the pneumatically actuated material where the light sources are embedded in. For physical objects, passive manipulations refer to purely physical control. For example, Babele (Appendix I, No.02) (see Table 3.3 (bottom-middle)) enables users to disassemble its lampshade directly by hand.

In addition, pre-setting the light sources or physical objects with a constant state is another way of passive manipulations. For the light sources, such kind of preset controls is analogical with the manual manipulation because that pre-setting the light sources requires an extra independent lighting interface anyway. For example, Soft Light (Appendix I, No.27) (see Table 3.3 (top-right)) has a constant warm light, which must be realised by firstly switching on the light manually. For the physical objects, pre-setting means to permanently keep the nonshape-changing objects with a constant state. For example, Resonance (Appendix I, No.23) (see Table 3.3 (bottom-right)) shows light projections on a fixed and flat wall whose shape is not tended to be changed.

\subsubsection{Attributes}

In this section, we investigate what kinds of attributes are used to trigger the light change and shape change. We classify four types of attributes: light, shape, spatiality, and material. Light attributes only apply to light sources, whereas shape and material attributes only apply to physical objects. Attributes in spatiality is utilised for deploying the relevant positions between light sources and physical objects.

\section{Light attributes}

Light attributes (see Table 3.4) are used to manipulate the light sources. In our sample, we distinguished two types of light attributes: one concerns the basic light properties for single light source, the other involves the fused light properties for composite light sources. For the basic light properties, the properties on/off, intensity, and colour are mostly used in our sample. For the other type of light attributes, some examples apply composite light sources to create dynamic luminous patterns that not only change multiple basic light properties but also change the distribution of the light sources in spatial dimension or the rhythm of the light change in time dimension.

The change of on/off is a basic way to switch light between two states: on or off. For example, in the case of Shape-shifting Lamp (see Table 3.4 (first-left)), when a user pulls the pneumatically actuated material, the light is turned on in response. 


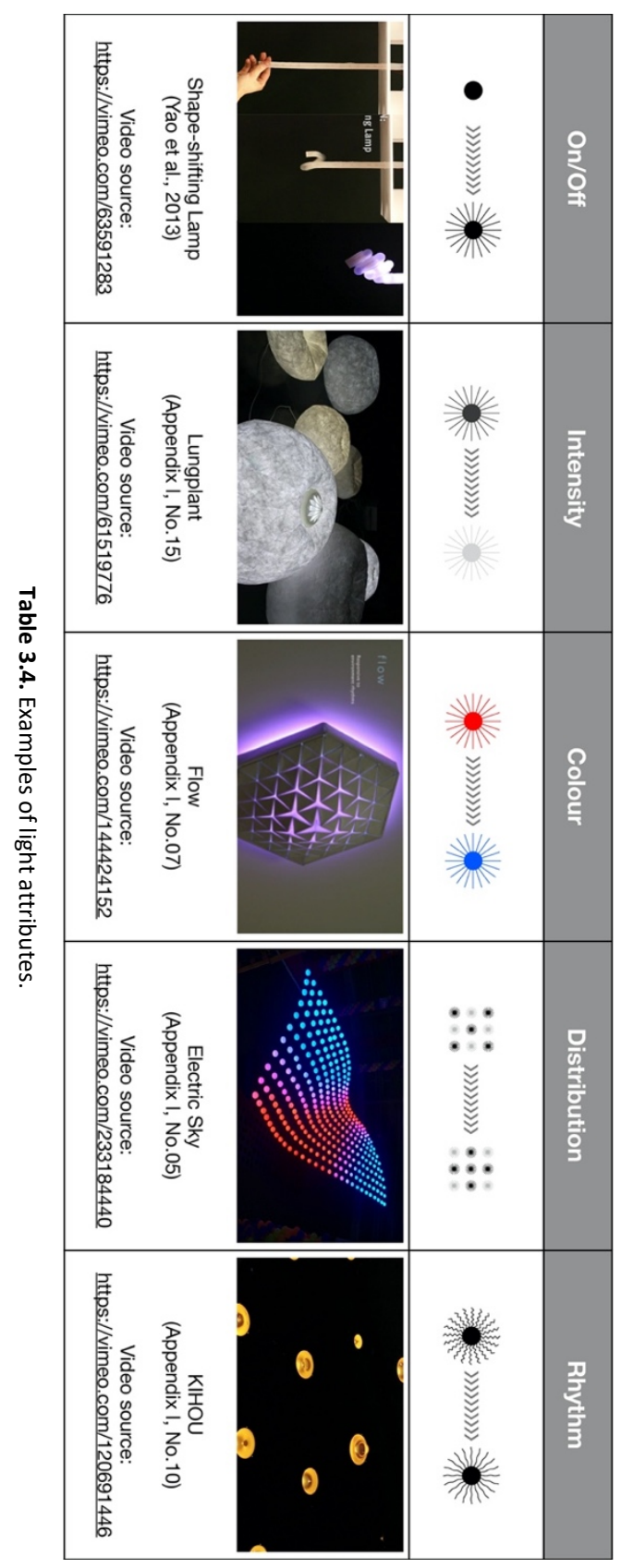


The change of intensity refers to the manipulation of the amount of brightness of the light. It provides only one dimension to manipulate the light along. For example, in the case of Lungplant (Appendix I, No.15) (see Table 3.4 (second from left)), every lamp can change the intensity of its light source with an inflating and deflating movement of its lampshade.

The change of colour includes the temperature change of light. It provides multiple dimensions of manipulating light. Colour change is widely used in our sample. For example, in the case of Flow (Appendix I, No.07) (see Table 3.4 (middle)), the colour change of its light source is caused by the response to different environmental conditions.

The distribution change is realised by changing the positions of the light sources' individual element with kinetic light fixtures. For example, Electric Sky (Appendix I, No.05) (see Table 3.4 (second from right)) changes the relevant positions of its LED elements to produce a dynamic light sculpture in three dimensions.

The change of rhythm refers to the change in on/off, intensity, colour, or distribution over time. For example, the intensities of the light sources of KIHOU (Appendix I, No.10) (see Table 3.4 (first from right)) repeat from bright to dim every 5 seconds.

\section{Shape attributes}

In SLS, we explicitly defined the shape attributes that refer to the properties of physical shape change. According to the framework of Rasmussen (Rasmussen et al., 2012), there are eight types of shape change in terms of: orientation, form, volume, texture, viscosity, spatiality, adding/subtracting, and permeability. Most of these types of shape change can be found in our sample, and listed in Table 3.5.

Changes in orientation distort the original shape through rotations or changes in direction, while preserving the recognizability of the original form. For example, Resonance (Appendix I, No.23) (see Table 3.5 (first from left)) distorts each of its light fixtures to create an overall wave-like shape change.

Form changes are defined by transformations that preserve the approximate volume of the shape while changing its overall form. For example, Lumio (Appendix I, No.14) (see Table 3.5 (second from left)) enables user to change the form of the lamp by folding and unfolding the lamp cover.

Volume changes the size of a physical object. For example, Lungplant (Appendix I, No.15) (see Table 3.5 (third from left)) deploys a fan on the top of its lampshade to produce an inflating and deflating movement. 


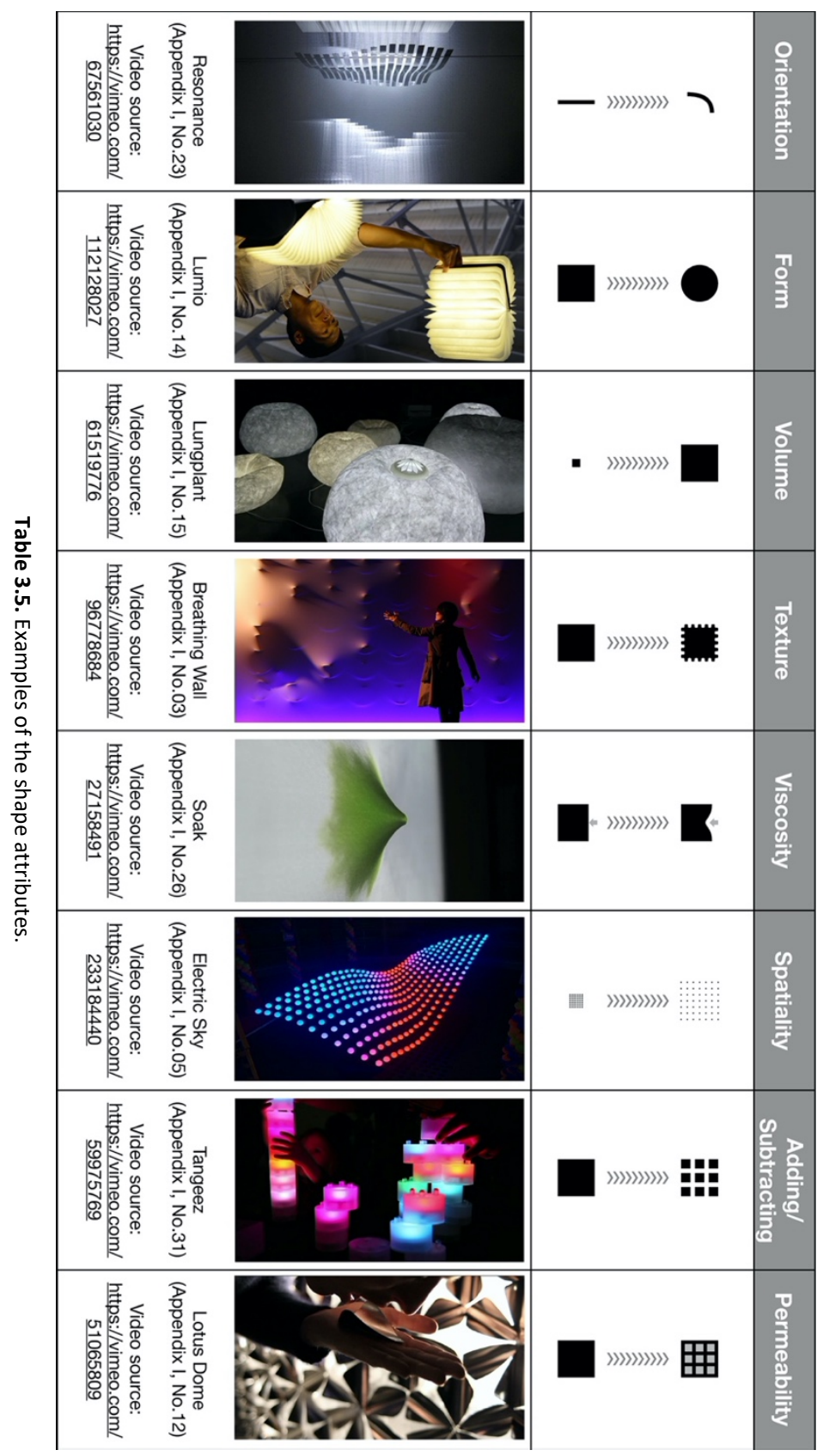


Textural changes are small changes on the surface of the shape that add visual and tactile properties without affecting the overall form. For example, Breathing Wall (Appendix I, No.03) (see Table 3.5 (forth from left)) shows a shape-changing "skin" with multiple raised patterns on a Spandex.

Changes in viscosity occurs when a physical surface is pressed but the original form is maintained. For example, when the user is poking the elastic fabric screen of Soak (Appendix I, No.26) (see Table 3.5 (fourth from right)), he/she can perceive the surface as shifting between loose and tight.

Changes in spatiality mean the illusion of shape change, which are caused by manipulating the position and/or shape of multiple elements in space. For example, the Electric Sky (Appendix I, No.05) (see Table 3.5 (third from left)) manipulates a matrix of LED elements to produce the kinetic sculpture of light.

Changes in adding or subtracting are achieved by uniting or dividing the elements with different manners, while being able to return to the original shape or shapes. For example, Tangeez (Appendix I, No.31) (see Table 3.5 (second from right)) provides a set of modular illuminated blocks for kids to build light into different shapes by hands.

Changes in permeability are defined by transformations, where the shape is perforated but able to return to its initial state. For example, Lotus (Appendix I, No.12) (see Table 3.5 (first from left)) shows different light gaps by opening and closing its aluminium flowers.
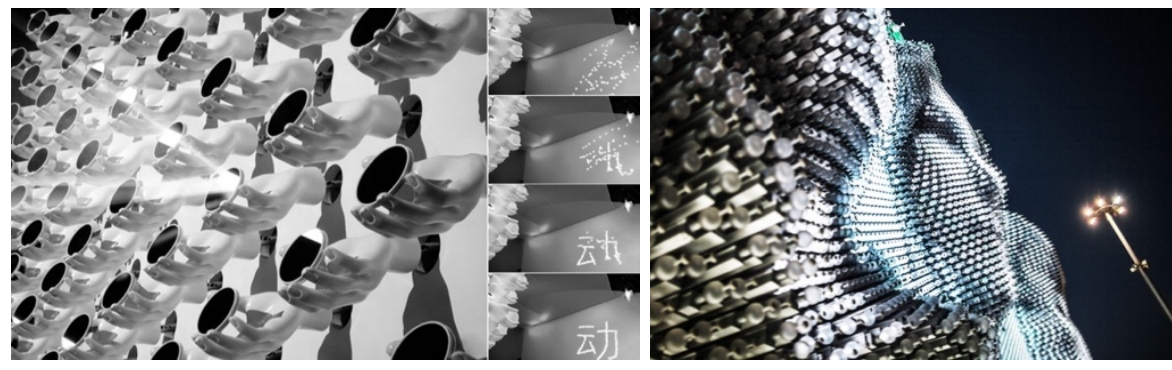

Figure 3.1. From left to right: MOBILITY (video source: https://vimeo.com/67559505); MegaFaces (video source: https://vimeo.com/99030884)

\section{Spatiality attributes}

In our sample, we also see some examples that manipulate the spatiality attributes by means of pre-setting the relevant positions between light sources and physical objects. The goal of manipulating spatiality attributes is to present the projections on a passive surface appropriately. For example, the spatial relations between light sources, mirrors, and wall in 
Mobility (Appendix I, No.18) (see Figure 3.1 (left)) are computationally designed to produce a projection of a Chinese character. That is different from the distribution changes in light attributes or the spatiality changes in shape attributes that is used to create the illusion of shape-changing luminous body. For example, MegaFaces (Appendix I, No.17) (see Figure 3.1 (right)) shows the illuminated human faces in three dimensions by changing the distributions of its light sources along with the spatiality of its light fixtures in shape.
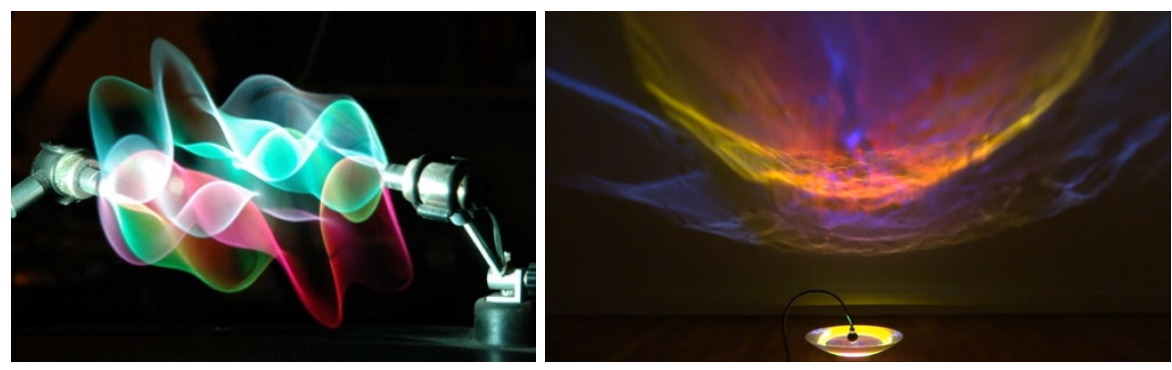

Figure 3.2. From left to right: String Waves (video source: https://vimeo.com/100402097); Eternal Sunset (video source: https://vimeo.com/191648808)

\section{Material attributes}

Material attributes are used for pre-setting the optical properties and appearance of the physical objects. These optical properties refer to the ability to change the propagation of light, such as reflectivity and transparency. For examples, String Waves (Appendix I, No.30) (see Figure 3.2 (left)) applies optical fibres to form the light in different shapes. The appearance in material attributes involve the properties (e.g., form, colour, pattern, texture, scale, etc.) that might influence the perception of the light projections. For example, Eternal Sunset (Appendix I, No.06) (see Figure 3.2 (right)) applies a dichroic filter floating and turning in the water to create sunset-like projections that cast on the wall. In this case, the colour of the dichroic filter, the form of the basin, the optical properties of the water, and even the appearance (flat and white) of the wall, together create the perception of sunset.

\subsubsection{Creating light patterns}

SLS combine shape change with light to create light patterns. According to the principle of light-object interactions, different deployments of light sources and physical objects produce different light patterns. In our sample, we saw six ways to deploy light sources and physical objects: emitting, diffusing, blocking, reflecting, refracting, and projecting.

Emitting creates initial light patterns, which are perceived directly from the light sources. This type of light pattern is produced by changing the spatiality attributes of multiple light sources. 


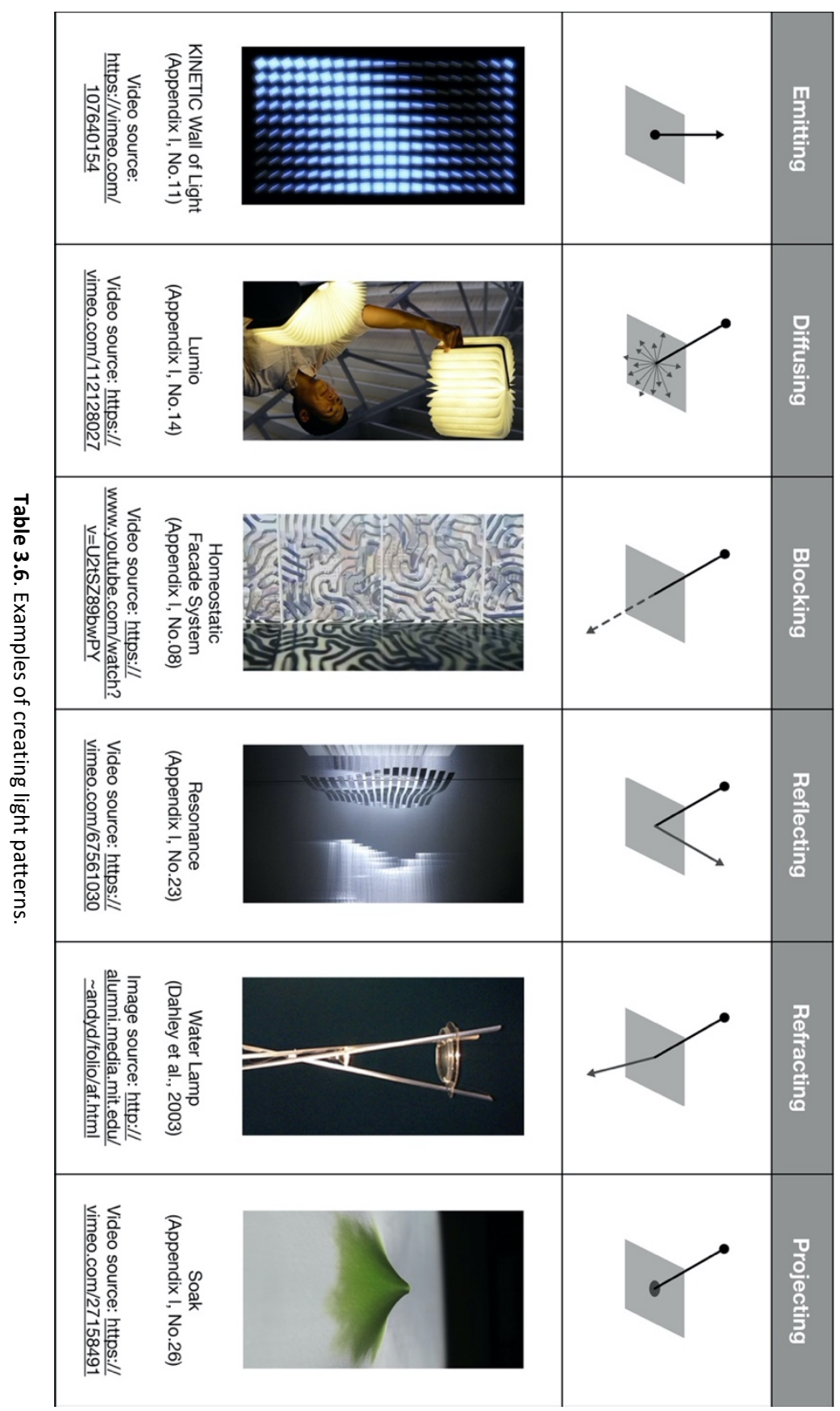


For example, KINETIC Wall of Light (Appendix I, No.11) (see Table 3.6 (first from left)) manipulates a matrix of LED panels to produce the kinetic sculpture of light in three dimensions.

Diffusing creates a luminous body by using a diffuser. For example, Lumio (Appendix I, No.14) (see Table 3.6 (second from left)) uses a foldable cover to produce a book-like lamp.

Blocking creates two types of light patterns, which are produced by using physical objects to block parts of the light from light sources. One type refers to light gaps, which are perceived on the shade, and the other type includes shadows, which are perceived on another surface. For example, Homeostatic Façade System (Appendix I, No.08) (see Table 3.6 (third from left)) provides a deformable façade that can automatically regulate the sunlight inside the building. The light gaps can be seen on its façade, while the shadows can be seen on the floor.

Reflecting also creates two types of light patterns, which are produced by using reflective physical objects that reflect part of the light emanating from the light sources used. One effect refers to reflected light patterns which are perceived on the reflector, and the other refers to reflected projections which are perceived on another surface. For example, Resonance (Appendix I, No.23) (see Table 3.6 (third from right)) uses kinetic body of mirrors to produce ever-changing light projections that cast on the wall.

Refracting creates two types of light patterns as well by using transparent physical objects to refract the light emanating from the light sources used. One type refers to refracted light patterns which are perceived on the refractor, and the other type refers to refracted projections which are perceived on another surface. For example, Water Lamp (see Table 3.6 (second from right)) deploys a small basin of water to create refracted light projections that cast on the ceiling.

Projecting creates luminous images, which are produced by utilizing a beamer. For example, Soak (Appendix I, No.26) (see Table 3.6 (first from right)) reacts the user that is touching on its elastic fabric with animated projections.

\subsection{Discussion of the Design Space for SLS}

This review analysed a sample of related examples and identified four categories of configurable design elements of SLS: light sources (sunlight, ambient natural light, traditional light bulbs, LEDs, OLEDs, smart light bulbs, and beamer), and physical objects (light fixtures, shades, diffusers, reflectors, refractors, environmental screens, and shape-changing screens); two ways of manipulating (active manipulations and passive manipulations) light sources and physical objects; the attributes (light, shape, material, and spatiality) for manipulating light 
sources and physical objects; the light patterns (initial light, luminous body, light gaps, shadows, diffused light, reflected light, reflected projections, refracted light, refracted projections, and luminous images) that are created by six ways (emitting, diffusing, reflecting, blocking, refracting, and projecting) of deploying light sources and physical objects. Together, these options indicate numerous possible alternatives for the implementation of specific SLS.

In this section, we will discuss the potential of alternative combinations as mentioned above in the context of Information Decoration. Also, we will demonstrate these opportunities by imagining alternative implementations of collected examples in the Information Decoration systems. Based on this discussion, we seek to outline the concrete features of SLS and update give a new, and more specific definition of SLS.

\subsubsection{Information Decoration}

Information Decoration is a special form of information displays, as its primary goal is not information but aesthetics. It stays in our background as a piece of art without obtrusiveness, providing information that we wish to be available at all times but in a way which can be ignored easily. By this analysis, we saw a big potential of applying SLS for Information Decoration.

In our sample, we saw some examples that are developed for representing information in an abstract and aesthetic way. For examples, in the case of Water Lamp (see Figure 3.3 (left)), the designers imagined that the refracted projections can be used to display the heartbeat of a loved one; Flow (Appendix I, No.07) (see Figure 3.3 (middle)) changes the colour of its light and the form of its origami lampshade to represent the environmental conditions; Gleamy (see Figure 3.3 (left)) portrays the data in an abstract and artistic way with different light patterns by manipulating the permeability (transparency) of its lampshade.
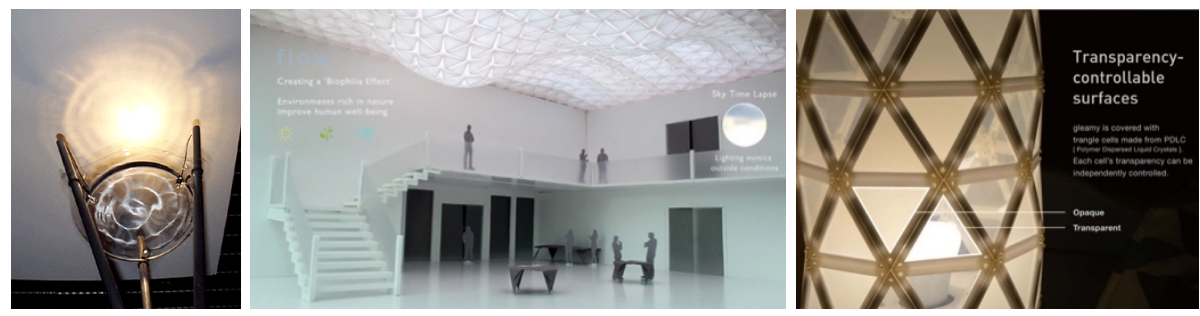

Figure 3.3. From left to right: Water Lamp (image source:

http://alumni.media.mit.edu/ andyd/folio/af.html); Flow (video source: https://vimeo.com/144424152); Gleamy (image source: http://seijincha.com/filter/Prototyping/Gleamy) 

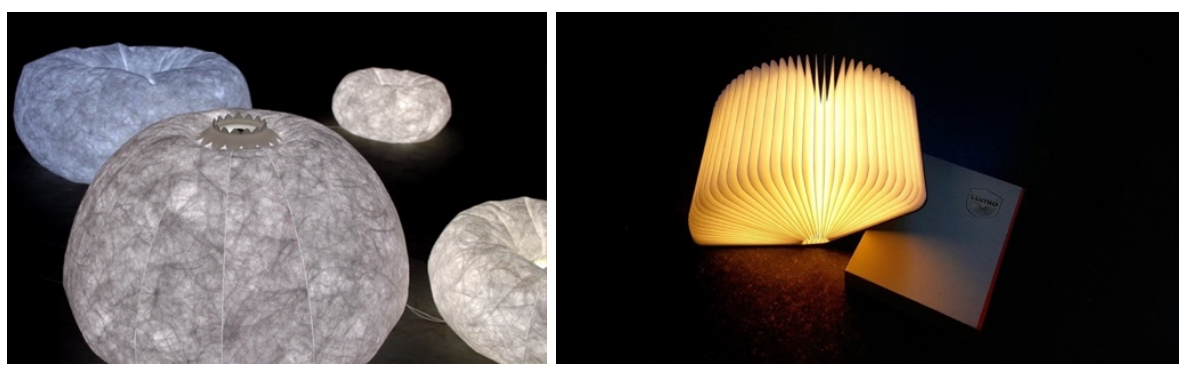

Figure 3.4. From left to right: Lungplant (video source: https://vimeo.com/61519776); Lumio (image source: https://vimeo.com/112128027)

In addition, we foresaw more possibilities of designing SLS for Information Decoration by imagining how to develop the examples in our sample into information displays. For example, imagine that the Lungplant (Appendix I, No.15) (see Figure 3.4 (right)) could simulate the breathing patterns of the patients, it might be much easier for nurses to be aware of the changes of the patients' situation rather than checking the data displayed on the equipment occasionally. In the rest parts of the section, we will introduce more examples like that to show the potentials of designing SLS for information.

\subsubsection{Manipulations}

In this review, we identified two types of manipulations (active manipulations and passive manipulations) on light sources and physical objects in SLS. These two types of manipulations can bring two benefits to the Information Decoration systems: one is providing multimodality displays of light and shape, the other is supporting user's tangible controls of light.

\section{Active manipulations provide multi-modality displays}

Active manipulations include both natural and automatic manipulations on light sources and shape-changing objects. They can provide multimodality displays of light representation and shape representation. This kind of multimodality displays can represent the same information in a rich way. For example, imagine that Lumio (Appendix I, No.14) could represent the indoor temperature information by both its form changes in shape and its colour changes in light. Moreover, the multimodality displays can represent rich information at the same time. For example, imagine that the Lumio could represent not only the indoor humidity information by its form change in shape but also the indoor temperature information by its colour change of light.

\section{Passive manipulations support tangible controls of light}


In SLS, passive manipulations on the shape-changing objects enable the user to play with light and create ever-changing light patterns. From this point of view, we believe that SLS can support users to alter the information representation of light by manipulating the relevant shape-changing objects in a tangible way. For example, All Lamp (Appendix I, No.01) (see Figure 3.5 (right)) allows the user to form it freely into different shapes. What if the different shapes referred to different types of information representation of light? When the lamp is formed as a circle, it represents the information of indoor humidity by changing its intensity in blue colour. Also, when the lamp is formed as a square, it represents the information of indoor temperature by changing its intensity in red colour.
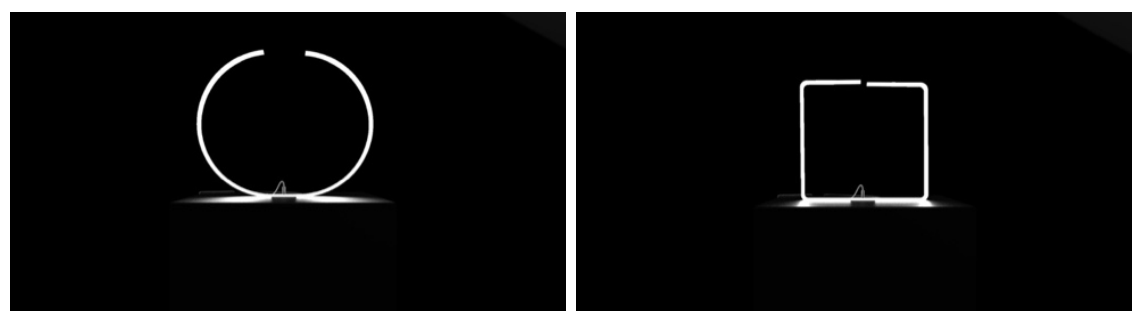

Figure 3.5. Two shapes of All Lamp (image source: http://tobiaslugmeier.com/work/allamp/)

\subsubsection{Attributes}

In this review, we identified four types of attributes (light attributes, shape attributes, spatiality attributes, and material attributes) for the manipulations on light sources and physical objects in SLS. In fact, all these attributes can be manipulated for information representation. However, it will be too complicated to do so. Considering that the focus of SLS is on applying shape-changing techniques to the lighting systems, we mainly concerned the uses of shape attributes and light attributes for encoding the information representations. Whereas, the spatiality attributes and material attributes are used for decorating the information representations.

\section{Attributes for representation}

The manipulations on light attributes and shape attributes are used for encoding the information representations. They can be active to provide multi-modality displays of light and shape. For example, what if the Lungplant (Appendix I, No.15) (see Figure 3.4 (right)) could simulate the breathing pattern of the patient by changing the volume of its shape as well as changing the intensity of its light. Also, the passive manipulation on shape attributes can be encoded with information to support tangible controls of light. For example, what if the user could check different types of information by tangibly forming the All Lamp (Appendix I, 
No.01) (see Figure 3.5) in different shapes. Moreover, it is also possible to encode information to the light attributes in a passive way. In this case, the information representation by means of shape change alters according to different manipulations of light. For example, imagine that KIHOU (Appendix I, No.10) (see Figure 3.6) could simulate the rain condition by its illuminated bubbling effect. What if it also allowed the user to select which city's rain condition he/she wants to check by changing the colour of its light. Red light means the rain condition local, or yellow light refers to the rain condition in another city.
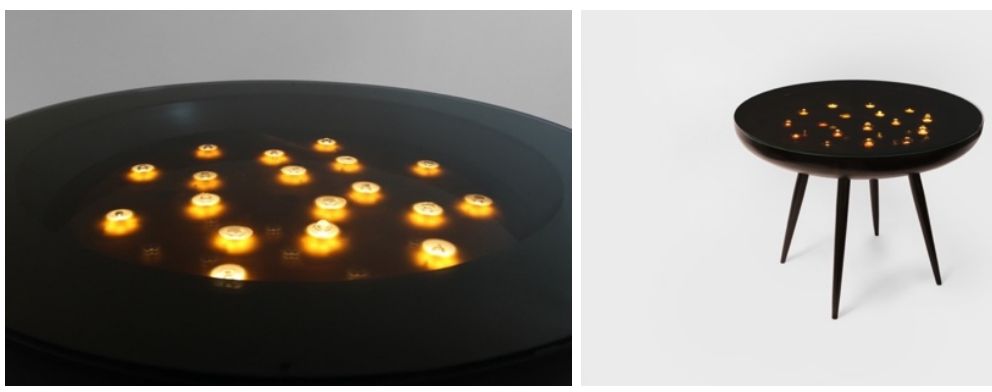

Figure 3.6. KIHOU (video source: https://vimeo.com/120691446)

\section{Attributes for decoration}

To make the meaningful light patterns be perceived aesthetically pleasing and environmentally appropriated, it is essential to consider the attributes that might influence on presenting the information representations of SLS. These attributes include the spatiality attributes and material attributes. For example, as we mentioned above, what if KIHOU (see Figure 3.6) could simulate the rain condition by its illuminated bubbling effect. Its round basin, randomly distributed bubbles, and translucent silicone together could provide the user with an aesthetical and subtle awareness of the raining information. Also, its table form can help such an Information Decoration be seamlessly integrated into the indoor environment.

\subsubsection{Light patterns}

SLS create meaningful light patterns which are the main carrier of information. According to where the user can perceive the light patterns, we identified three kinds of Information Decoration by means of SLS: initial light displays, sculpted light displays, and projected light displays. In this section, we will discuss about the potentials of using these three kinds of Information Decoration.

\section{Initial light displays}


The initial light displays are perceived directly from the light sources. Such kind of information displays is composed with multiple light sources and shape-changing light fixtures. These light sources and light fixtures can be easily manipulated and freely combined to create diverse incident light patterns. However, perceiving initial light displays directly on the light sources in close distance can be obtrusive. Therefore, we think that the Information Decoration by means of initial light displays might be more fitted in the public space, where is broaden enough for the user to ignore the information displays easily. For example, the Drone Light Shows (Appendix I, No.04) (see Figure 3.7 (left)) are potentially able to portray the information either in detailed (e.g., words) or in general (e.g., abstract patterns). Otherwise, it's better to employ subdued light sources (e.g., OLEDs) when implementing the initial light displays for Information Decoration. For example, imagine that Manta Rhei (Appendix I, No.16) (see Figure3. 7 (right)) could sync its dynamic effect with the liveness of a social occasion by sensing the noisy level surrounded it.
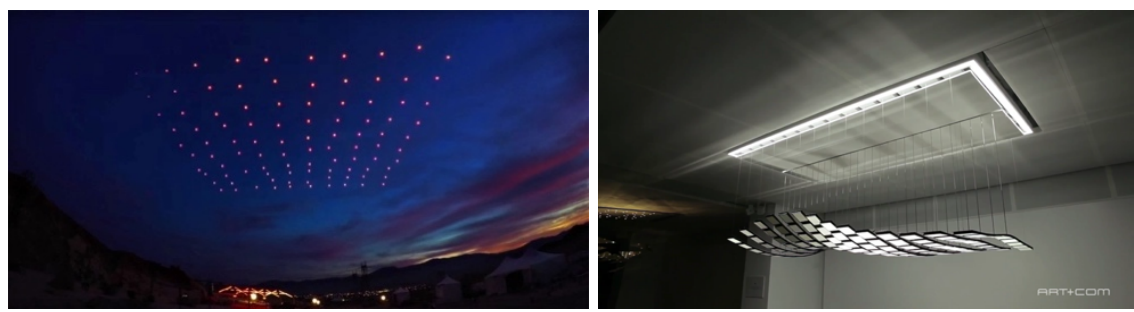

Figure 3.7. From left to right: Drone Light Shows (video source: https://www.youtube.com/watch?v=aOd4-T_p5fA); Manta Rhei (video source: https://vimeo.com/46289124)
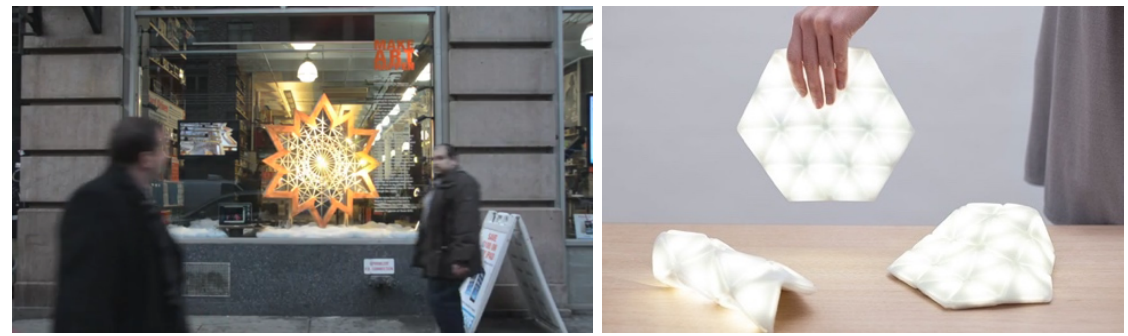

Figure 3.8. From left to right: Light Waves (video source: https://vimeo.com/82379175); Kangaroo Light (video source: https://vimeo.com/95299083)

\section{Sculpted light displays}

The sculpted light displays are only perceived on the shape-changing objects. They could be diffused light, light gaps, reflected light, or refracted light. Comparing with the initial light displays, the Information Decoration by means of sculpted light can be subtler. Therefore, the sculpted light displays can be either applied in the public space to convey information gently 
or positioned closer to the user in the personal context without obtrusiveness. For example, Light Waves (Appendix I, No.20) (see Figure 3.8 (left)) attracts the passer-by with the dynamic reflected light. What if it could show what kinds of products are on sale by different colours of the light?

Also, the sculpted light displays can support tangible interactions when the user wants to alter the information displays by hand. For example, Kangaroo Light (Appendix I, No.09) (see Figure 3.8 (right)) is a deformable lamp. What if it could represent different types of information depending on different forms the user shapes it into?

\section{Projected light displays}

The projected light displays can be perceived on the non-shape-changing objects where the sculpted light shines on. They could be presented as shadow, reflected light projection, or refracted light projection. These projected light displays are potentially able to represent abstract information and to be calmly integrated into the environment as decorations. Also, they can expand the perceivable range of SLS, in which the user not only can perceive the projected light displays on non-shape-changing objects but also can perceive the sculpted light displays on shape-changing objects. For example, imagine that the Resonance (Appendix I, No.23) (see Figure 3.9 (left)) could represent the outdoor temperature by the colour change of its light sources and the outdoor wind speed by the shape change of its kinetic mirrors. In this case, the user can check the whole outdoor climate by looking at either the light projection on the wall or the reflected light on the shape-changing mirrors.
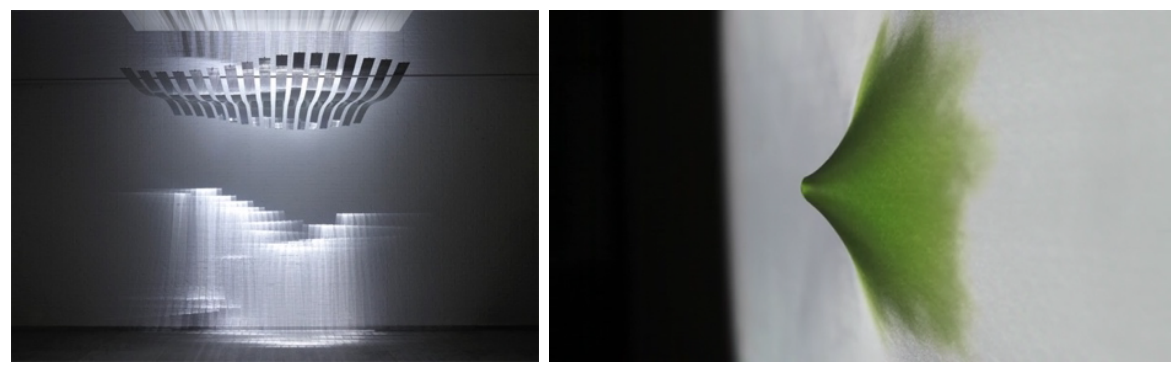

Figure 3.9. From left to right: Resonance (video source: https://vimeo.com/67561030); Soak (video source: https://vimeo.com/27158491)

Also, the projected light displays can be perceived on a shape-changing screen, where a beamer projects the luminous images on. This kind of projected light displays can provide rich information representation and support the user in altering the projected light displays in a tangible way. For example, what if the Soak (Appendix I, No.26) (see Figure 3.9 (right)) only presented the detailed information when the user touches the flexible screen and showed decorative patterns in an abstract way for the rest of time. 
However, positioning the strong initial light from beamer outside the user's view can be a challenge with the risk of obtrusiveness caused by the exposing beamer.

\subsubsection{Definition of SLS}

In this discussion, we explored the potentials of applying SLS for Information Decoration. Also, we specified the use of different manipulations, attributes, and light patterns in the context of Information Decoration. Based on that, we give a new and more specific definition of SLS, especially the (tangible) interaction opportunities are added to the definition:

SLS are lighting systems (installations) that combine shape change with light. They can provide multi-modality displays of light and shape by actively manipulating the light sources and shapechanging objects. Also, they can support tangible controls of light for the user to alter the information displays by directly manipulating the shape-changing objects. SLS are different with the other informative or artistic lighting systems by the features of aesthetically pleasing, subtly meaningful, tangibly interactive, and environmentally appropriated.

\subsection{An Overview of SLS Building Blocks}

To provide a systematic overview of the alternatives in designing SLS for Information Decoration, we summarised SLS building blocks by three clusters of design elements: four basic components for construction, three types of attributes for manipulation, and three kinds of light patterns for information mapping.

In this section, we will introduce these design elements in detail. Also, we will use these design elements to describe the design cases (Timer Lamp and Timer Shade) we have introduced in the previous chapter. Timer Lamp (section 2.3) represents the meeting progress by automatically changing the distribution of its light sources and the form of its lampshade (see Figure 3.10 (left)). Timer Shade (section 2.4) portrays the meeting progress by the shadows cast on the table. These shadows are produced by deploying a shape-changing coiled cap to partly block the light from a constant light source (see Figure 3.10 (right)).

\subsubsection{Four basic components for construction}

In the earlier review of SLS (see section 3.2), we identified two different ways to manipulate light sources and physical objects: active manipulations and passive manipulations. Based on that, we can distinguish light sources and physical objects into four basic component 
categories: Active Lights, Passive Lights, Active Objects, and Passive Objects (Wu, Van Essen, \& Eggen, 2016).
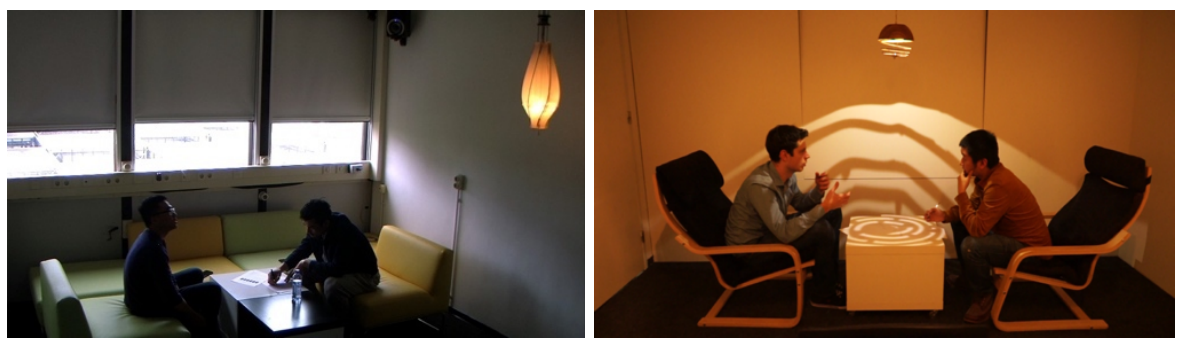

Figure 3.10. From left to right: Timer Lamp (video source: https://vimeo.com/274437412); Timer Shade (video source: https://vimeo.com/274438399)

Active Lights refer to light sources that can automatically change their light properties. For natural lights, the changes of their appearances follow the laws of nature, which cannot be changed by human beings. For artificial light sources, the changes of Active Lights can be manipulated directly by computer systems. For example, Timer Lamp (section 2.3) implements a moving LED-equipped pad (Active Lights), which represents the time information by automatically changing its position inside the lamp body.

Passive Lights indicate the light sources that have constant light properties until people change them via an extra independent switch or smart device. For example, to turn on/off a lamp with a switch, or to change the colour of Phillips Hue via an app on a smartphone. Also, in the case of Timer Shade (section 2.4), we implemented a Passive Light source (a power LED) to provide constant brightness during the meeting.

Active Objects are shape-changing objects that can automatically change their physical shapes. Such an automatic shape-changing can be actuated by a computer program. For example, the lampshade of Timer Lamp (section 2.3) can represent the time information by its automatic form changes. Also, the shape change of Active Objects could be animated by specific properties of the object's material. For example, which kind of material (e.g., shape memory alloys) could be available for the coiled cap of Timer Shade (section 2.4) to return its initial shape by itself?

Passive Objects refer to physical objects that have constant physical properties until people change them intentionally by touch. They can be used to just show the projected light patterns. For example, Timer Shade (section 2.4) deploys a table to show shadows. Also, Passive Objects could be implemented as a tangible interface for manipulating light. For example, what if we 


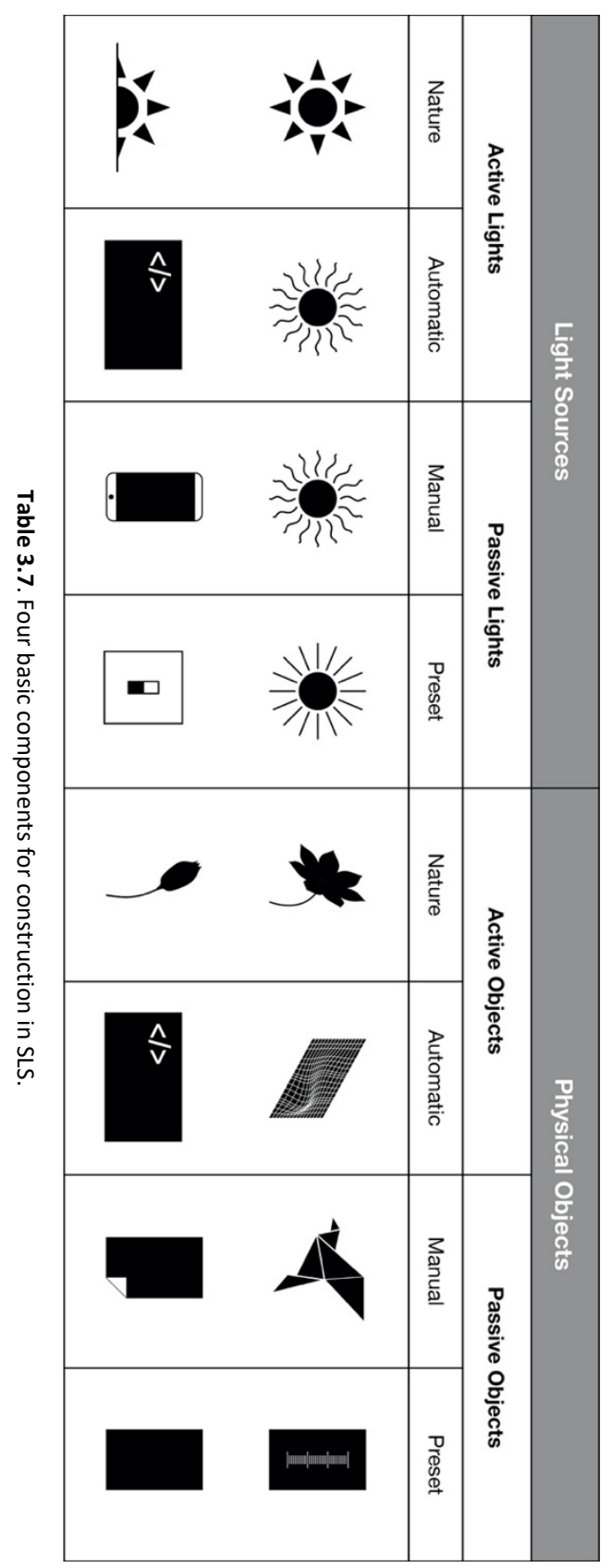


designed Timer Lamp (section 2.3) as a portable lamp that positioned on the table so that the user could set the time by changing the form of the lamp tangibly without using an extra tablet.

\subsubsection{Three types of attributes for manipulation}

As we have discussed in the last section, there are two ways of manipulating the attributes of light sources and physical objects. The light attributes and shape attributes are essential in SLS for information encoding. While the spatiality attributes and material attributes are extra and are only needed for presenting the information displays appropriately. Based on that, we specified what attributes can be used for the four components mentioned above.

Light Attributes are used to encode the manipulations on Active Lights or Passive Lights with information. According to the previous analysis, the Light Attributes include: on/off, intensity, colour, rhythm, and distribution. These properties can be utilised individually. For example, the light sources of Timer Lamp (section 2.3) are only manipulated in distribution. Also, the properties in Light Attributes can be combined to produce rich light representation. For example, in the case of Timer Lamp (section 2.3), what if its light sources could reduce their intensities along with the bottom-up movement?

Shape Attributes are manipulated to provide information representations by means of shape change. According to the framework of Rasmussen, there are eight types of shape change: orientation, form, volume, texture, viscosity, spatiality, adding/subtracting, and permeability. These attributes are usually integrated in the shape change. For example, in the case of Timer Shade (section 2.4), the shape change of the coiled cap is a combination of form and permeability.

Extra Attributes define the non-encoding properties that are manipulated for presenting the light patterns in an appropriate and decorative way. The most important extra attribute concerns the relevant positions between the light sources and the physical objects that are implemented in SLS. For instance, Timer Shade (section 2.4) is fixed above the table with a certain distance to make sure of that the shadows can be perceived clearly. The other optional extra attributes relate to the properties of the physical objects that are used to decorate the information representation. There are many kinds of these attributes such as: pattern, colour, texture, transparency, reflectivity, etc. For example, in the case of Timer Shade (section 2.4), what if we added some decorative pattern on the white table to help user to interpret the shadow displays better? 


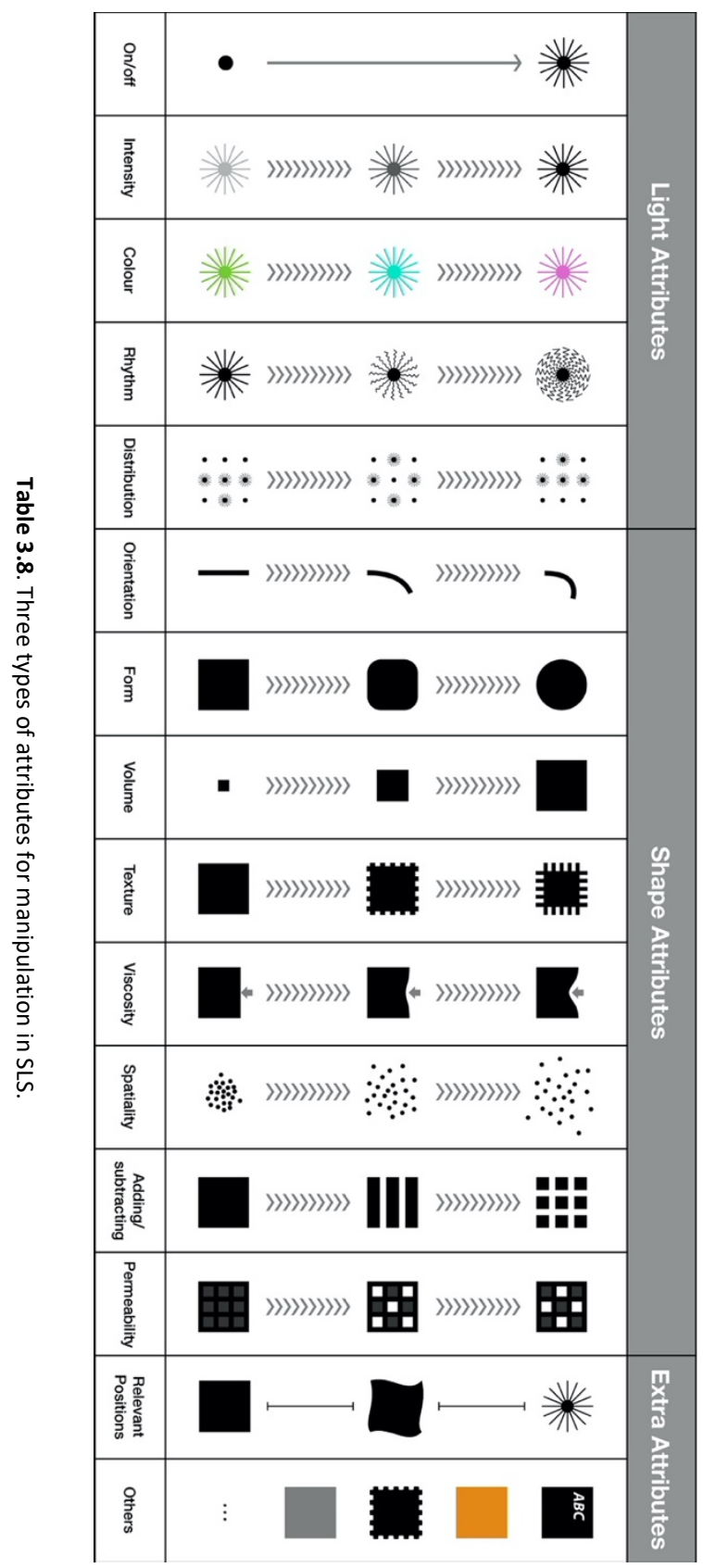




\subsubsection{Three kinds of light patterns for information mapping}

In our previous discussion, we identified three kinds of Information Decoration by means of SLS. They are initial light displays, sculpted light displays, and projected light displays. Also, we have indicated different applications in different context. Based on that, we define three kinds of light patterns in SLS for Information Decoration: initial light patterns, sculpted light patterns, and projected light patterns.

Initial Light Patterns can be only perceived on the light sources. They are created by deploying multiple light sources and light fixtures. Initial Light Patterns are capable to display information either in detailed level or abstract level. However, this kind of light patterns is only meant to be perceived in distance. Otherwise, they might cause obtrusiveness which is not the intention of designing SLS for Information Decoration.

Sculpted Light Patterns are only meant to be perceived on the shape-changing objects. They could be luminous body, light gaps, diffused light, reflected light, and refracted light. Depending on whether the shape-changing objects are active or passive, the sculpted light can be deployed in different scenarios. The sculpted light perceived on the active shape-changing objects are deployed as multi-modality displays of light and shape in the user's peripheral attention. For example, Timer Lamp (section 2.3) presents a shape-changing luminous body which combines the form change in shape and the distribution change in light. Also, the Sculpted Light Patterns can be perceived on the passive shape-changing objects that are deployed to support user's tangible manipulation of light representation. For example, what if the Timer Lamp was started by moving the LED-equipped pad down to the bottom manually?

Projected Light Patterns can be perceived on Passive Objects which are intentionally fixed. It could be shadows, reflected projections, or refracted projections which are produced by using Active Objects to sculpt the initial light. For example, in the case of Timer Shade (section 2.4), the shadows on the table are Projected Light Patterns. Also, Projected Light Patterns can be some luminous images that are produced by a beamer. In this case, Projected Light Patterns will be perceived on a shape-changing object, which is implemented as a tangible interface for the user to manipulate the projected light patterns. For example, Tangible CityScape (see Figure 3.11 (left)) augments the shape change of its interactive tangible interfaces with projected image. 


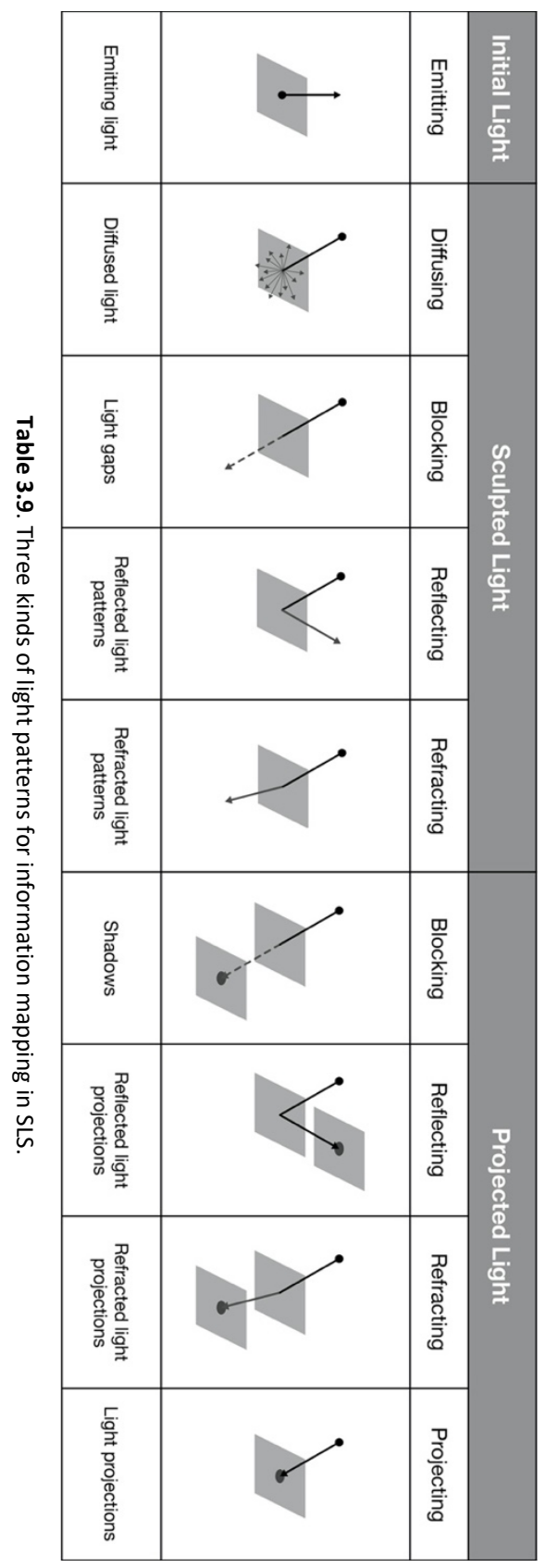




\subsection{Four Styles of SLS}

The SLS building blocks present a systematic way to implement SLS with three categories of design elements, which are components for construction, attributes for manipulation, and light patterns for information mapping. These design elements provide diverse opportunities to design SLS for Information Decoration. In this section, we will discuss four typical styles of SLS, which are linked to the four basic combinations of light sources and physical objects. These four styles of SLS can potentially fit in different scenarios.
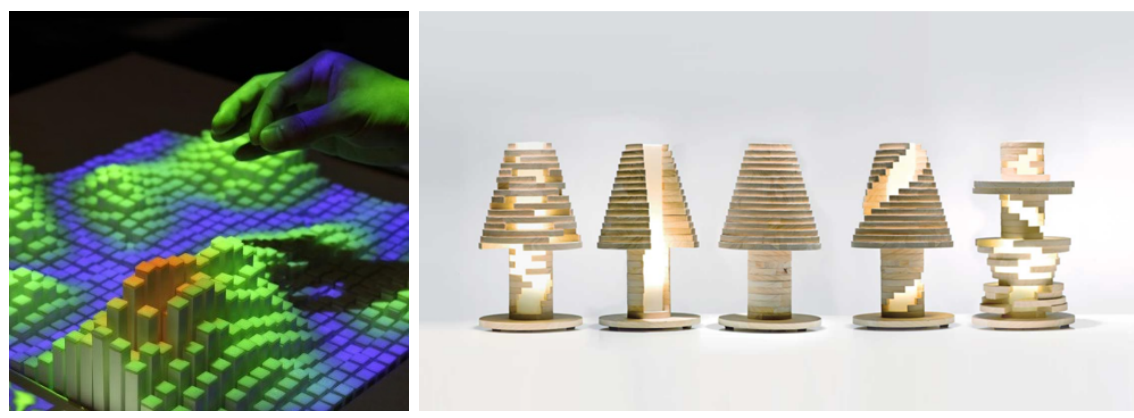

Figure 3.11. From left to right: Tangible CityScape (image source: http://tangible.media.mit.edu/project/tangible-cityscape); Babele (image source: http://www.designmid.it/eng/babele.php)

Active SLS are implemented by combining Active Lights with Active Objects to present lively expression of light and shape as whole. They can be used to represent one piece of information in a rich (redundant) way. For example, in the case of Timer Lamp (section 2.3), the time information is represented by both the form change in shape and the light change in position. Also, Active SLS can potentially represent two types of information individually. For example, imagine that the Timer Shade (section 2.4) could automatically change the colour of its light to red once someone else is making appointment to use the meeting room soon.

Objects-driven SLS are implemented by combining Passive Lights with Active Objects. This type of SLS could be applied to a context where the initial light is not suggested to change automatically or frequently, for example, the functional lighting in a working context. In the case of Timer Shade (section 2.4), we implemented a Passive Light source which has a constant intensity while automatically changing the form of the coiled cap (Active Object) to produce shadows that cast on a white table (Passive Object).

Light-driven SLS are implemented by combining Active Lights with Passive Object. In this case, light sources change their properties automatically to react to users that tangibly manipulate Passive Objects. The changes of light with Active Lights and the change of shape with Passive 
Objects together produce the Sculpted Light Patterns or the projected light patterns. For example, a deformable lamp originally shows the current temperature outdoors by the colour of its light. When people change the shape of its deformable cover, the colour of its light changes accordingly and shows the temperature information indoors.

Passive SLS are implemented by combining Passive Lights and Passive Objects. This style of SLS enables users to create information displays by themselves through manually manipulating the light sources and physical objects. For example, Babele (Appendix I, No.02) (see Figure 3.11 (right)) has a constant light source, and it enables the user to disassemble the form of its lampshade (Passive Objects) manually to create different patterns of light gaps. What if the light gaps could mean something in private?

\subsection{Discussion and Conclusion}

In this chapter, we first presented an analysis of the state-of-the-art examples of SLS that combine light with shape change. As a result, we identified diverse alternatives to implement SLS in terms of light sources, physical objects, manipulations, attributes, and creating light patterns. Then, we discussed these alternatives in the context of Information Decoration. Based on that, we presented a systematic overview of SLS building blocks by three clusters of design elements: four basic components, three types of attributes, and three kinds of light patterns. Furthermore, we discussed the combinations of these elements and proposed four styles of SLS which fit in five different scenarios. The SLS building blocks helped us in reflecting on our design explorations. We believe that an understanding of SLS in the form of these building blocks can provide practitioners with a systematic overview to describe, compare, and critique SLS concepts. However, the set of building blocks might be incomplete since they were generated from a limited number of relevant examples. Also, the use of these building blocks in design practice is still unknown. To address these two issues, at the end of this chapter, we will discuss the limitation of the SLS building blocks, and we will formulate conclusions on the potential of using the SLS building blocks.

\subsubsection{Limitation of the SLS building blocks}

The SLS building blocks are developed from an analysis of the existing examples that combine light with shape change. Most of these examples are design projects that are collected online by searching relevant keywords. However, we expect other examples beyond our collection. The main reason is that the description of the design projects can be very subjective and not include the keywords we used. In this sense, we saw limitation that our sample might be 
incomplete, which might restrict the inclusivity of the SLS building blocks. In other words, there might be other design elements that are essential to be considered in the implementation of SLS. As a matter of fact, we found out another essential component (section 4.4.1) of SLS afterwards by reflecting the use of these building blocks in practice. Furthermore, we integrated all the founded design elements into a SLS Framework, which will be introduced in the next chapter.

\subsubsection{Potential of using the SLS building blocks}

In section 3.4, we described the design cases of Timer Lamp (section 2.3) and Timer Shade (section 2.4) with different design elements introduced in the SLS building blocks. The Timer Lamp is an Active SLS, while the Timer Shade is an Objects-driven SLS. From here, it is natural to reflect on these two concepts by thinking about the possibilities of using other design alternatives provided in the SLS building blocks. For example, is it possible to design another style of SLS (e.g., Light-driven SLS) that can represent the time information? Also, is it possible to apply other types of shape attributes or light attributes for the information representation of time?

We can foresee that SLS building blocks can support designers in diverging concepts with rich possibilities, while a framework for them to organise/converge their concepts with a holistic point of view is still missing. This framework should help designers in describing, comparing, and critiquing SLS concepts intuitively at a higher level. In this way, designers can not only recognise which design alternatives are used/unused but also which design elements are used/unused. In the next chapter, we will introduce this framework and its visual representation.

To conclude, in this chapter we proposed SLS building blocks, which are developed from an analysis of a sample of existing examples that combine light with shape change. These building blocks can help in reflecting on design concepts, however they might be still incomplete and too abstract to support designers in developing SLS in the entire design process. To fill this gap, we carried out a second iteration of design explorations, from which we proposed a framework of SLS. For designers, the framework outlines SLS with a complete set of design elements, which can help them to organise diverse possible SLS concepts. For researchers, the framework provides a basis for discussing open research questions of SLS in future studies. 
CHAPTER 4.

SLS Framework 
In the previous chapter, we presented an overview of SLS building blocks based on an analysis of relevant existing SLS examples. These building blocks helped us to systematically describe two early design cases (Timer Lamp and Timer Shade), and enabled us to identify different styles of SLS. In this chapter, we carry out a second iteration of design explorations in order to delve into yet unexplored SLS styles. We developed two new SLS prototypes (Reflective Clock and Time Glow). By reflecting on the explorative design process, we identified a new SLS component and uncovered new interaction possibilities for SLS.

Based on our understanding of the components of SLS and reflections on our design explorations, we propose an SLS Framework in section 4.4. Based on this framework and the interaction opportunities we identified, we propose a potential design space that applies SLS for an adaptive and interactive rather than a perceive-only Information Decoration system.

\subsection{Intentions}

The primary goal of the design explorations is to demonstrate the styles of SLS that we had not explored yet. In the previous design explorations (see chapter 2), Timer Lamp represents a style of Active SLS, combining Active Lights and Active Objects without involving Passive Objects; while Timer Shade shows a style of Objects-driven SLS, which combines Passive Lights, Active Objects, and Passive Objects. In this second round of design explorations, we demonstrate two other styles of SLS by means of the Reflective Clock and Timer Glow design explorations, respectively. Reflective Clock presents a variant of Active SLS, which combines Active Lights, Active Objects, and includes Passive Objects; Time Glow shows a style of Lightsdriven SLS, which combines Active Lights and Passive Objects.

The secondary goal of these design explorations is to reflect on the completeness of the SLS building blocks. The SLS building blocks were formulated through analysing a number of existing SLS examples. Although they provide vocabularies which allow to describe Timer Lamp and Timer Shade, these two design cases only represent two possible styles of SLS. In the new design explorations, we explore two other styles of SLS. By doing so, we mean to find out the elements of SLS that are ignored in the previous review but worth to be highlighted to facilitate the design of SLS in practice.

The last goal of the design explorations is to discover new possible interactions with SLS. In our previous design explorations, we have shown one type of interaction, which enables the user to alter the information displays on the data processing level. In the second round of design explorations, we continued to discover new possible interactions in SLS. In particular, 
we aim to explore the alternative ways to integrate the SLS into the user's context in an interactive way.

\subsection{Reflective Clock}
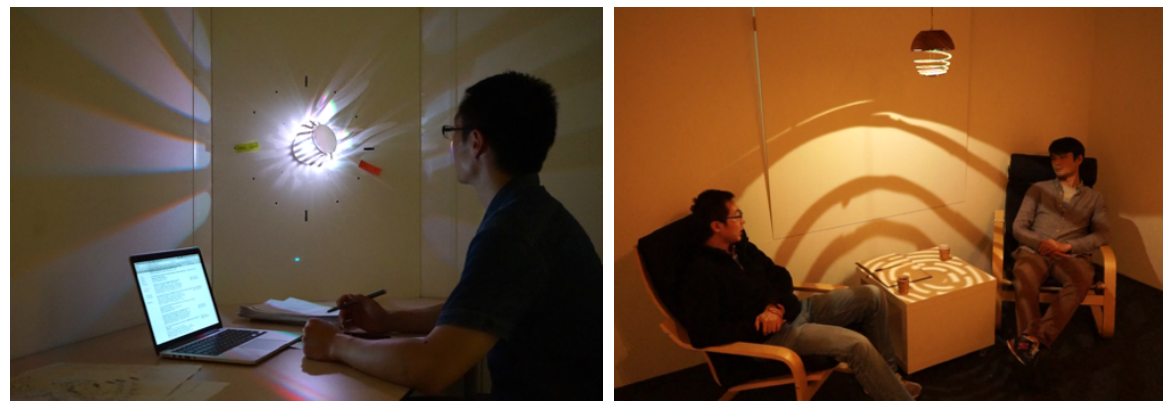

Figure 4.1. (Left) Scenario of Reflective Clock; (Right) The concept of Timer Shade.
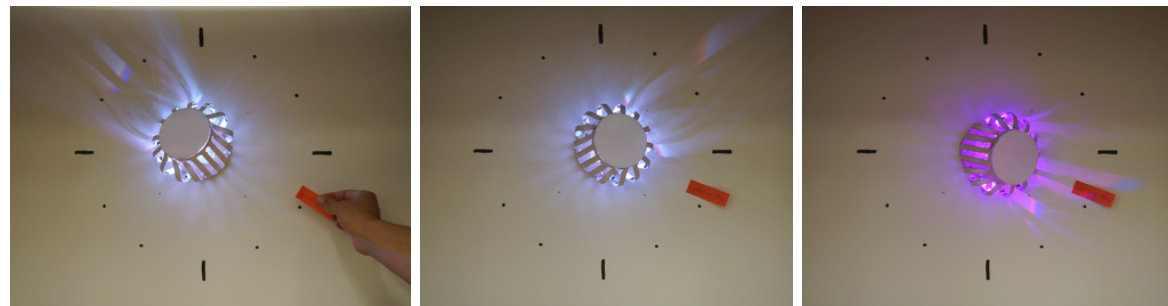

Figure 4.2. The interaction in Reflective Clock: (Left) making schedule by placing a sticky-note on the corresponding scales on the whiteboard; (Middle) normal state of Reflective Clock; (Right) changing colour while the reflected light projections are approaching the sticky-note.

Reflective Clock $^{11}$ (see Figure 4.1 (left)) is a decorative wall lamp in a personal office. It is designed to support people being aware of the time information in a subtle way. Reflective Clock consists of a flexible cage, which is made by mirror sheet, and an RGB LED as the only light source for this lamp, which is fixed on the centre of a scaled whiteboard. Normally, Reflective Clock produces subtle reflected light projections that cast on the whiteboard to show general time information (see Figure 4.2 (middle)). The user can place his/her stickynotes to the corresponding scales on the board as physical reminders (see Figure 4.2 (left)). When the reflected light projections are approaching the sticky-note, the colour of them will

\footnotetext{
${ }^{11}$ Jiang Wu (2015). Reflective Clock. Retrieved on 29.06.2019 from https://vimeo.com/274517223
} 
gradually change to the colour of the note (see Figure 4.2 (right)) to notify the user that it is time for a scheduled activity.

Comparing with Timer Shade ${ }^{12}$ (see Figure 4.1 (right)), Reflective Clock presents a better way of integrating the SLS in its surroundings. On the one hand, the scales can help people to interpret the meaningful reflected light projections on the whiteboard. However, in the case of Timer Shade, we only utilised an ordinary table to show the shadows which are considered as too subtle to be understood easily. On the other hand, placing the sticky-notes on the board merges a daily performance into the input for the user to alter the information displays intuitively. It can be more immersive than deploying the meaningful light patterns on the user's surroundings only (e.g., the shadows on the table in the case of Timer Shade).

\subsubsection{Highlighting Preset Objects}

Reflective Clock presents a type of Active SLS. More specifically, it combines three types of SLS components, which are Active Objects, Active Lights, and Passive Objects. The Active Object refers to its flexible cage, which automatically changes the orientation of its shape according to the change of time. The Active Lights refer to the RGB LED, which automatically changes its colour at some moments that are set by the user. The Passive Objects involve the scaled whiteboard, which is pre-set by us to present the reflected light projections, and the coloured sticky-notes, which are pre-set by the user to indicate the moments he/she need notifications by changing the colour of the reflected light projections.

In the case of Reflective Clock, we see that pre-setting the physical objects in SLS can potentially provide the user with a better experience of the Information Decoration. The designers can implement such pre-setting. For example, we utilised the whiteboard to show clear light projections, and the scales on the whiteboard to help the user interpret and give meaning to the light patterns. Also, the pre-setting of SLS can be implemented by the user. For example, the user placed the sticky-notes to decide when a notification is needed (e.g., placing the sticky-note in the scales on the whiteboard) and how intrusive the notification can be (e.g., choosing a red sticky-note to mark an important event).

To inform these new design possibilities mentioned above to design practice, we considered highlighting Preset Objects as a new component of SLS. We initially defined Preset Objects as the physical objects that are pre-set to support the user better perceiving and interpreting the

\footnotetext{
${ }^{12}$ Jiang Wu (2014). Timer Shade. Retrieved on 29.06.2019 from https://vimeo.com/274438399
} 
information displays of SLS. The implementation of Preset Objects can be realised either by designers or by users.

\subsubsection{The interaction alters the information representation}

Reflective Clock demonstrates a new type of interaction which enables the user to alter the information representation of SLS. It is realised by changing the mapping between the data and the light attributes of the Active Lights. This interaction is different with the ones in Timer Lamp ${ }^{13}$ and Timer Shade. The interactions in Timer Lamp and Timer Shade enable the user to input data, whereas neither the light attributes nor the shape attributes for data mappings are affected. For example, no matter the user sets the duration of a meeting to 30 minutes or to one hour, Timer Lamp always presents a luminous body that moves its light sources bottom-up inside a deformable lampshade with a constant property of light. If Timer Lamp could also change its colour of light according to the user's preference, then we consider this interaction to alter the information representation.

The interaction that alters the information representation can also change the mapping between the data and the shape attributes of Active Objects. When we were experimenting with the light effect of Reflective Clock, we found that the reflected light projections can be shorter or longer depends on the deformation of the mirrored flexible cage. From this, we thought: what if the length of the reflected light projections can be altered according to the position of the sticky-note on the whiteboard? For example, when the user places a sticky-note outside the scales on the whiteboard, it means the event wrote on the note is very important. In this situation, the reflected light projections will be lengthened to be more noticeable when they are approaching the sticky-note.

\subsection{Time Glow}

Time Glow (see Figure 4.3) is also designed to support people being aware of the time information however in a domestic context. It possesses a fabric cover (see Figure 4.3 (middle)) and 60 RGB LEDs (see Figure 4.3 (right)), which are fixed along a circled frame behind the fabric cover. These 60 LEDs are distributed like the scales of the second in a clock. There is a steel ball at the centre of the fabric cover for the user to touch. With a super magnet, we fix this steel ball with a rod, which is connected with a DC motor used to detect the deformation

\footnotetext{
${ }^{13}$ Jiang Wu (2014). Timer Lamp. Retrieved on 29.06.2019 from https://vimeo.com/274437412
} 
of the fabric cover. According to the deformation of the fabric cover, Time Glow activates different numbers of LEDs. For example, poking in the fabric cover activates 15 LEDs, keeping the fabric cover activates 9 LEDs, and pulling out the fabric cover activates 3 LEDs. These activated RGB LEDs move like real hands on a clock. The LEDs that create the red coloured light represent the information of the hour, The LEDs that create the blue coloured light represent information of the minutes, and the LEDs that create the green coloured light represents information of the seconds.
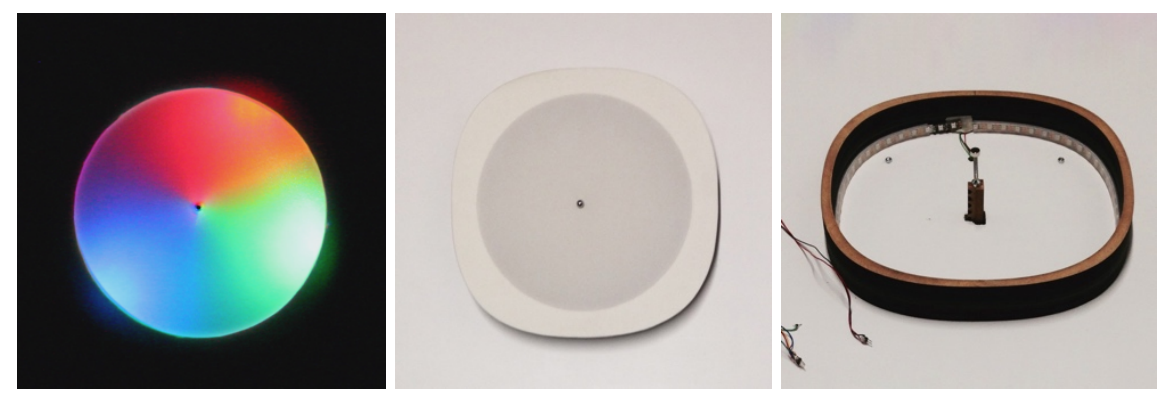

Figure 4.3. (Left) The Information Decoration of Time Glow when the fabric cover is poked in; (Middle) The fabric cover and the steel ball at its centre; (Right) The 60 RGB LEDs distributed along the circled frame behind the fabric cover.
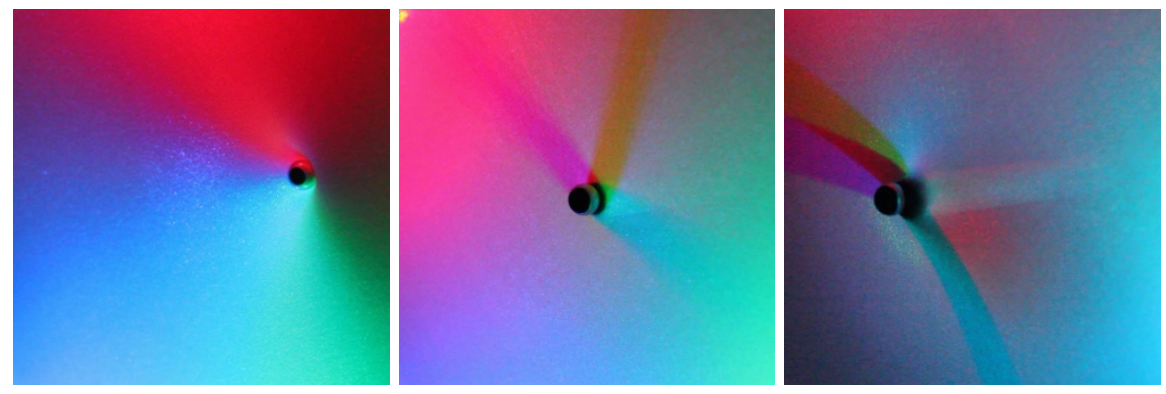

Figure 4.4. The three different modes of Information Decoration of Time Glow. (Left) The colour wash light flows that shows 16:51:42 on a sunk fabric cover; (Middle) The subtle coloured shadows flow that shows 16:52:03 on a flat fabric cover; (Right) The clear coloured shadows tick that shows 16:52:54 on a raised fabric cover.

By manipulating the fabric cover of Time Glow, the user can perceive three kinds of light pattern which present real-time information in three different modes. The first mode portrays the subtle flow of time by colour wash light (see Figure 4.4 (left)). It more functions as a decorative lamp, in which the fabric is sunk and rendered by 15 LEDs (five LEDs for each colour of Red, Green, and Blue). The second mode presents the time information with subtle coloured shadows (see Figure 4.4 (middle)). In this mode, the fabric is flat, and 9 LEDs (three LEDs for each colour of Red, Green, and Blue) are activated. The third mode presents clear 
coloured shadows that tick like a clock (see Figure 4.4 (right)). In this mode, the fabric is raised and rendered by only three LEDs (one LEDs for each colour of Red, Green, and Blue).

\subsubsection{Distinguishing Passive Objects and Preset Objects}

Time Glow presents an example of Lights-driven SLS, which combines Active Lights and Passive Objects. The Active Lights refer to the 60 RGB LEDs, which automatically change their distributions and colours. Passive Objects refer to the fabric cover, whose form can be tangibly manipulated to switch different modes of information displays according to the user's different needs of being aware of the time information.

The fabric cover of Time Glow demonstrates a typical Passive Objects in SLS. It is very different from Preset Objects that are implemented in Reflective Clock. On the one hand, manipulating the fabric cover alters the information displays of SLS in real time. For example, the user can pull out or poke in the fabric cover to change the mode of information portrays based on his/her preference. While pre-setting the sticky-notes in the case of Reflective Clock is not intended to change the information displays immediately. On the other hand, the manipulation of the fabric cover is realised through shape-changing, whereas pre-setting the sticky-notes follows the user's daily activity which is not imitated by shape-changing.

Therefore, we redefine Passive Objects as the shape-changing objects that are implemented for the user to alter the information (re)presentation of the SLS in real time. Passive Objects are mainly implemented to support tangible interactions which enable the user to alter the information (re)presentations of SLS via shape-changing interfaces. Such shape-changing interfaces merge the input and the output of the information presentation. For example, the fabric in the case of Time Glow is implemented to enable the user to manipulate the meaningful light patterns shown on the fabric itself.

Also, we specify Preset Objects as the non-shape-changing objects that are pre-set by either the designers or the users to support a better perception or interpretation of the information displays in SLS. Preset Objects are mainly implemented to optimise the perception of the information displays in SLS. For example, the scales on the whiteboard in the case of Reflective Clock was intended to be a reference for the user to get the time information from perceiving the reflected light projections. Also, Preset Objects can be implemented to enable the user to modify the information (re)presentation of SLS through the tangible interactions that are wellintegrated with the context of SLS. For example, using the sticky-notes to set a physical reminder is an inherent behaviour in the office domain. 
By these two new definitions, we distinguish Passive Objects and Preset Objects from two perspectives. On the one hand, manipulating Passive Objects only concerns the shape attributes, while pre-setting Preset Objects can involve multiple extra attributes (e.g., spatiality, patterns, materials, colours, forms, etc.). On the other hand, although both Passive Objects and Preset Objects are manipulable by the users, the resulted feedback is perceived in different ways. For manipulating Passive Objects, the user perceives the resulting feedback in real time. However, the feedback generated by manipulating Preset Objects is not intended to be perceived immediately. For example, in the case of Reflective Clock, placing a sticky-note on the whiteboard happens much earlier than the moment when the user perceives the colour changes of the reflected light projections.

\subsubsection{The interaction alters the information presentation}

Time Glow presents a new interaction (see Figure 4.5) which enables the user to alter the information presentation of SLS. Realising such type of interaction requires SLS to provide more than two types of light patterns for the user to select based on his/her preferences. For example, Time Glow can present the time information by either coloured shadows or diffused colour wash light. This interaction is different from the one in Reflective Clock which only changes the colour mappings but always presents the time information by one type of light pattern (reflected light projections).
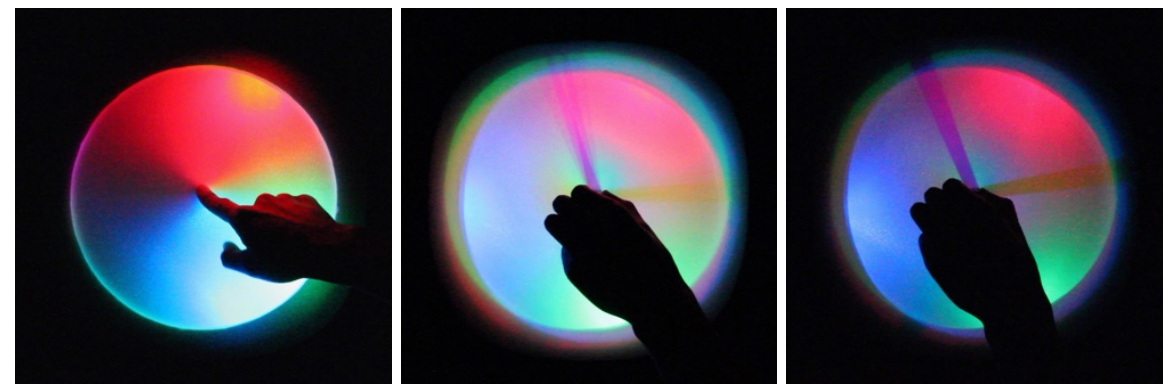

Figure 4.5. The interaction in Time Glow. (Left) poking in the centre of the fabric cover to perceive colour wash light; (Middle) keeping the fabric cover flat to perceive subtle coloured shadows; (Right) pulling out the fabric cover to perceive clear coloured shadows.

The interaction that alters the information presentation also can occur in Active SLS. In this case, Active Lights and Active Objects automatically change their attributes to produce different types of light patterns to present the information in different manners. Imagine what happens if Time Glow can actively switch its different types of information presentation by sensing the openness of the user. For example, when the user is focusing on his/her task, colour wash light will be presented to decorate the atmosphere in the background; when the 
user is approaching Time Glow, coloured shadows will smoothly emerge to show the clear time information.

Additionally, the interaction altering the information presentation can happen in Objectsdriven SLS as well. In the case of Timer Shade, we implemented a shape-changing coiled cap to produce meaningful shadows that cast on a white table. During the user test, some participants indicated that the contrast of the shadows on the table is too sharp, especially while they were reading or writing. So, what if the transparency of the coiled cap is manipulable? For example, when the user is reading or writing, the shadows will fade out through increasing the transparency of the coiled cap.

\subsection{A Framework for SLS}

In the previous sections, we have introduced two new design cases of SLS, which are Reflective Clock and Time Glow. These two design cases helped us to reflect on the completeness of the SLS building blocks. As results, we redefined the component of Passive Objects and highlighted a new SLS component called Preset Objects. In this section, we present a framework for SLS that integrates all the design elements of SLS. Also, we present a visualization of this framework to support designers in developing SLS with a holistic point of view.

The SLS Framework (see Figure 4.6) provides a systematic overview that presents and relates all the design elements of SLS. These elements include five basic components, three types of attributes, three kinds of light patterns, and four design styles. In Figure 4.6, all the design elements of SLS are represented abstractly. On the one hand, it emphasizes the interconnections rather than the alternatives of the SLS components, attributes and light patterns. By this representation, we aim to stimulate designers to think about SLS concepts at a higher level than just trying out different design alternatives, which are presented in the tables of the SLS building blocks (section 3.3). On the other hand, this representation of the SLS Framework can provide practitioners with a basic template to visualize SLS. For instance, Figure 4.7 shows four different styles of SLS based on the visual representation of the SLS Framework.

Firstly, the five basic components are Active Lights (the black diamonds in Figure 4.6), Passive Lights (the grey diamonds in Figure 4.6), Active Objects (the black wavy line in Figure 4.6), Passive Objects (the grey wavy line in Figure 4.6), and Preset Objects (the black dash line in Figure 4.6). The definitions of Active Lights, Passive Lights and Active Lights are the same as the ones we have introduced in section 3.3.1. While, the definitions of Passive Objects and Preset Objects can be found in section 4.3.1. 


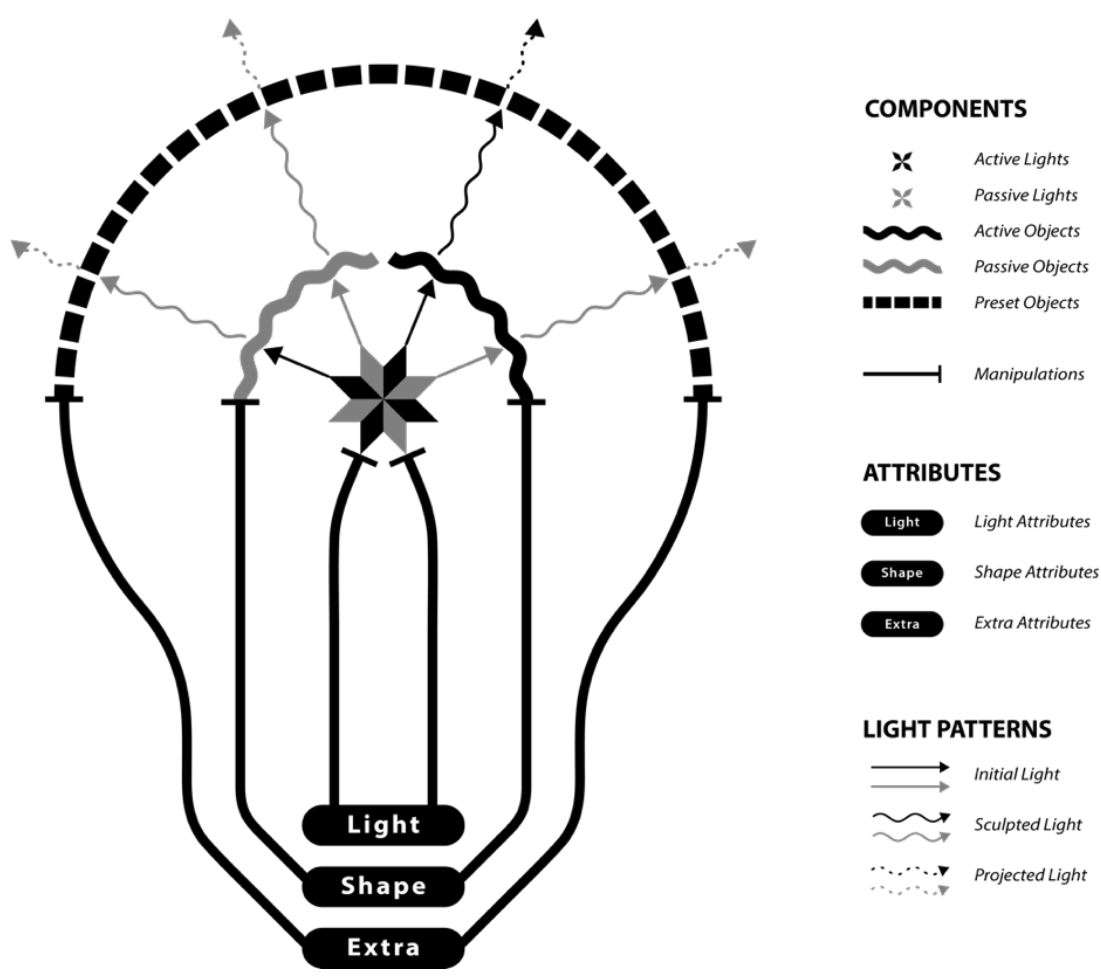

Figure 4.6. The visual representation of the SLS Framework. In this image, the black/grey diamonds refer to Active/Passive Lights; the black wavy lines refer to Active Objects; the grey wavy lines refer to Passive Objects; the black dash line refer to Preset Objects; the T-shape lines refer to manipulations; the three capsules refer to Light Attributes, Shape Attributes, or Extra Attributes, respectively; the black/grey straight arrows refer to Initial Light from Active/Passive Lights; the black/grey wavy arrows refer to Sculpted Light produced by combining Active/Passive Lights with Active/Passive objects; the black/grey dash arrows refer to Projected Light showed on Preset Objects.

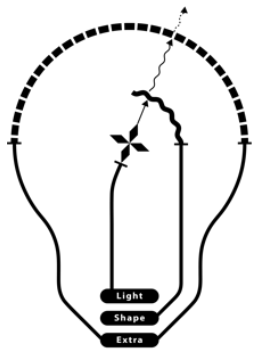

Active SLS

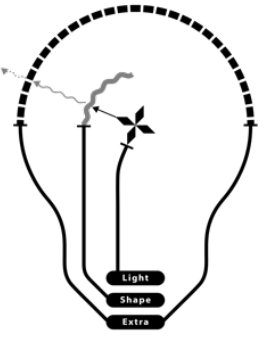

Lights-driven SLS

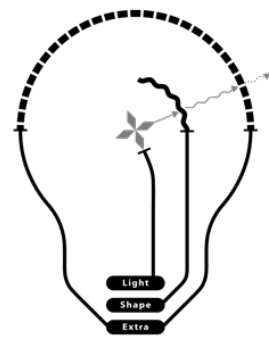

Objects-driven SLS

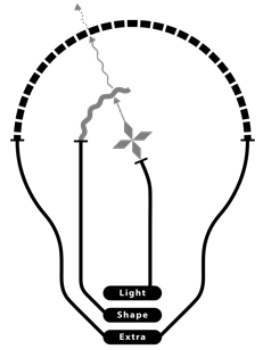

Passive SLS

Figure 4.7. The visual representation of the four styles of SLS (Sculpting Light Systems). From left to right: Active SLS combine Active Lights with Active Objects; Lights-driven SLS combine Active Lights with Passive Objects; Objects-driven SLS combine Passive Lights with Active Objects; and Passive SLS combine Passive Objects with Passive Objects. 
Secondly, the three types of attributes (the three capsules in Figure 4.6) are Light Attributes, Shape Attributes, and Extra Attributes. For Light Attributes and Shape Attributes, we have introduced their definitions and alternatives in section 3.3.2. While, for Extra Attributes, we highlight the potentials of manipulating Preset Objects to support the user not only better perceiving but also better interpreting the information displays of SLS.

Thirdly, the three kinds of light patterns are Initial Light (the black/grey straight arrows in Figure 4.6), Sculpted Light (the black/grey wavy arrows in Figure 4.6), and Projected Light (the black/grey dash arrows in Figure 4.6). The definitions of these light patterns have been introduced in section 3.3.3. Also, we visually distinguish the light patterns that are perceived from active components and from passive components. By this visualisation, we mean to stimulate designers for recognising more possibilities in creating a same kind of light patterns.

Lastly, the four design styles (see Figure 4.7) include Active SLS, Lights-driven SLS, Objectsdriven SLS, and Passive SLS. The definitions of these four styles of SLS are the same with the ones we have introduced in section 3.4. It is noticeable, however, that Preset Objects are not essential for any style of SLS. They are involved only when the Projected Lights are applied for information representation.

\subsection{Designing Adaptive SLS for Information Decoration}

In the first and the second rounds of design explorations, we have presented three kinds of interaction in SLS. These interactions respectively intervene with the information displays of SLS on the level of data processing, information representation, and information presentation. From this, we see many possibilities of applying SLS for an adaptive and interactive rather than a perceive-only Information Decoration system.

Firstly, the interaction in Time Lamp or Timer Shade is meant to define the data processing of the information displays. By this kind of interaction, the user can define the displayed information according to his/her needs. For example, if we enabled the user to define the meaning of the scales (e.g., 30 minutes per circle) on the whiteboard, the moving reflected light projections of Reflective Clock would also be able to support time management in the informal meeting scenario.

Secondly, Reflective Clock presents an interaction that modifies the representation level of the information displays in SLS. This kind of interaction can support the user encoding his/her preferred Information Decoration by modifying the mapping between the data and the representation of light or shape. For example, some participants indicated that they do not like the bottom-up movement of Timer Lamp to represent the time passing. Instead, they 
suggested the top-down movement using the metaphor of gravity. There's no need to argue which mapping is more appropriate for representing the time passing. However, it would be better to enable the user to define his/her preferred mapping beforehand.

Lastly, the interaction in Time Glow alters the information displays at the presentation level. In this kind of interaction, SLS can present the same information by different alternative meaningful light patterns. Thanks to this, it becomes possible to adapt SLS to the user's changing needs for perceiving the information displays. For example, the user can select the mode of coloured shadows to perceive clear time information. Also, the user can select the mode of colour wash light to fade out the information displays of time in the background.

\subsection{Conclusion}

In this chapter, we have presented two new explorations of designing SLS for Information Decoration. Together with our earlier explorations (see chapter 2), we have demonstrated three basic styles of SLS which are Active SLS by Timer Lamp and Reflective Clock, Objectsdriven SLS by Timer Shade, and Lights-driven SLS by Timer Shade. By reflecting on these two design explorations, we highlighted a new component in SLS which is Preset Objects and redefined the component of Passive Objects. Based on that, we propose a complete SLS Framework, and present a visualisation of the framework.

In this chapter, we also discovered two new interaction possibilities for designing SLS for Information Decoration. One intervenes the information representation of SLS, which concerns the mapping between data and the light attributes or shape attributes. The other alters the information presentation of SLS, which involves the mapping between data and the light patterns. These two types of interaction are different from the one presented in Timer Lamp and Timer Shade, which only manipulates the data processing of SLS. Through these three kinds of interaction, we uncovered a potential design space of applying SLS for an adaptive and interactive rather than a perceive-only Information Decoration system. To provide a systematic understanding of this new design space, in the next chapter we will propose an SLS Interaction Model. 
SLS Interaction Model 
In the previous chapters, we formulated a SLS Framework which presents a systematic way to construct lighting systems that combine light with shape-changing. The Framework identified five basic components, three types of attributes, three kinds of light patterns, and four styles of SLS. Based on the framework and our design explorations, we recognize opportunities to systematically identify different levels of interaction with SLS.

To understand this new design space, in this chapter we propose a SLS Interaction Model, which provides designers with a coherent picture of what is possible when developing an adaptive SLS for Information Decoration. More specifically, designers can use our Interaction Model as a mechanism to describe, discover and compare their design concepts and to come to well-founded design decisions.

\subsection{Introduction}

In this chapter, we first introduce the information flow of SLS, which illustrates how raw data is transformed into the Physical View through four steps. Then we add four interaction loops to this information flow to complete the SLS Interaction Model. These interaction loops indicate four kinds of interaction between SLS and the user and/or the environment. After that, we show the alternative techniques to realize the mentioned information flow and interaction loops. At last, we validate the use of our Interaction Model by applying it to the development of the design of Timer Lamp, which is the first hands-on design case through which we acquired new knowledge about designing SLS.

\subsection{Information Flow}

In this section, we propose the information flow in SLS. We describe the process of how to transform raw data into an informative SLS. This information flow is developed based on a "visualisation reference model" (see Figure 5.1). By adapting our concept of SLS based on this existing mechanism, we mainly redefined the transformation steps by using the new concepts introduced in our SLS Framework.

\subsubsection{The existing information visualisation pipeline}

A "visualisation reference model" (see Figure 5.1) or an "information visualisation pipeline" was firstly proposed by Card et al. (Card, Mackinlay, \& Shneiderman, 1999), and then refined by Chi and Riedle (Ed Huai-Hsin Chi \& Riedl, 2002) and Carpendale (Carpendale, 1999). Most of these conceptual models are primarily focusing on conventional setups for displaying 
information with intangible components on a computer screen. However, as we have seen, SLS provide multi-modal information displays with tangible components of light sources and physical objects.

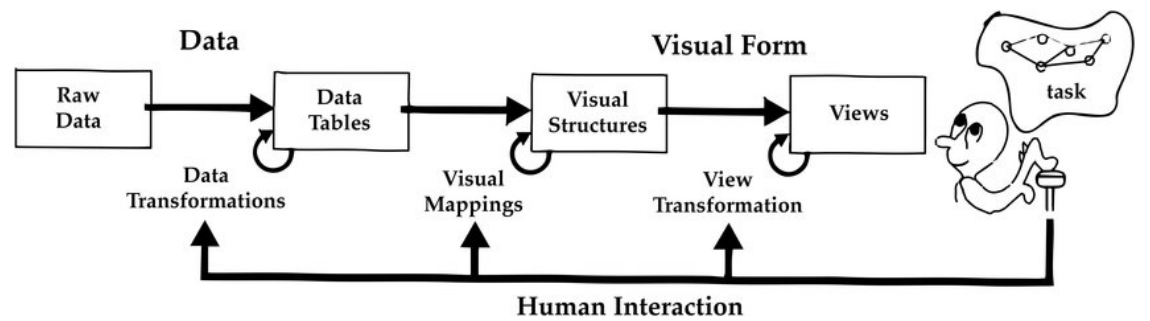

Figure 5.1: Visualisation reference model (Card et al., 1999).

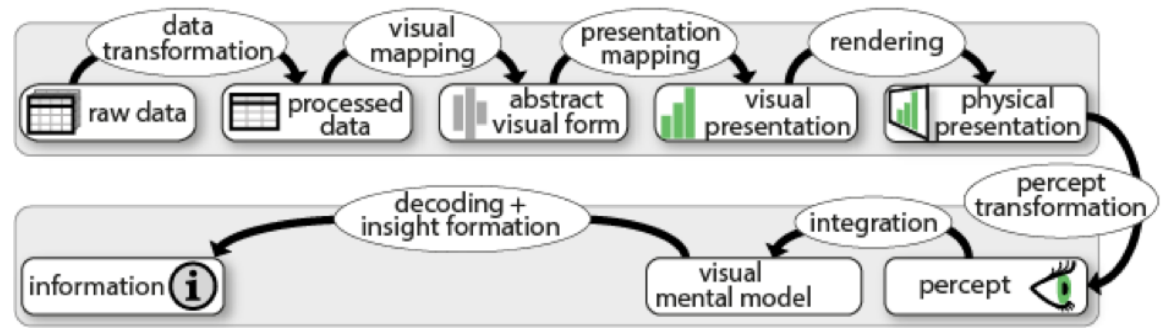

Figure 5.2. The adapted information pipeline of Jansen and Dragicevic.

Recently, Jansen and Dragicevic (Jansen \& Dragicevic, 2013) developed an adapted information pipeline (see Figure 5.2), attempting to also capture more non-conventional setups. This adapted information pipeline simply describes the presentation mapping as overlaying the abstract visual forms into a visual presentation. However, there are other ways to do so in the real world. Particularly, in our case of SLS, combining the abstract visual forms of light and physical shape always produces light patterns which are more than painting a physical form with a particular colour of light. For this reason, we propose to model the information flow by using the adapted information pipeline of Jansen and Dragicevic in the context of SLS. Also, we redefine the four transformation steps by using terminology in line with our SLS Framework.

\subsubsection{Information flow within SLS}

Inspired by the concepts of the information visualisation pipeline mentioned above, we modelled the information flow particularly for SLS (see Figure 5.3). Next, we will introduce this information flow by explaining its four transformation steps with a hypothetical example. In this example, we present an imaginary SLS system that can portray two types of information: 
the temperature difference between indoor and outdoor; the relative humidity indoor compared to the 'healthy' state. This imaginary SLS will be demonstrated by a set of figures, which are generated by using three different computer-aided design tools: Rhinoceros ${ }^{14}$, Grasshopper $^{15}$, and Keyshot ${ }^{16}$.

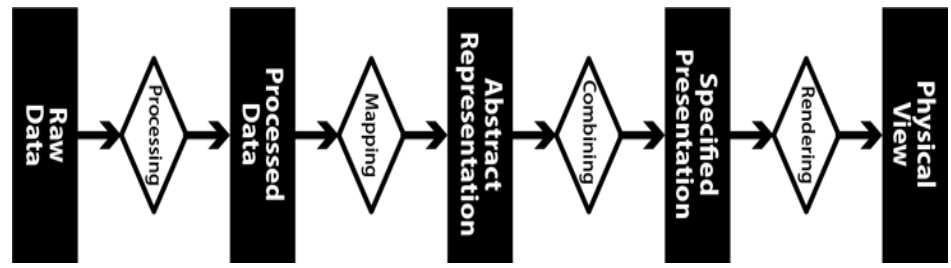

Figure 5.3. The information flow of SLS (inspired by Jansen and Dragicevic).

\section{Processing}

Firstly, the Raw Data is processed into a data format that is suitable for information representation. This can include compiling data from several sources, filtering and aggregating the data to suit the designer's questions, and making the data compatible with the representation technique used in the next stage. The Processed Data are the outcome of this step.

In our hypothetical example, the raw data are provided by two different data sources. The outdoor temperature $\left(t_{1}\right)$ is acquired from the Yahoo Weather API by filtering the items that relate to the local temperature. While the indoor temperature $\left(t_{0}\right)$ and indoor humidity $\left(h_{1}\right)$ are respectively captured by a temperature sensor and a moisture sensor placed on a wall of the user's drawing room. Moreover, we define the healthy humidity indoor $\left(h_{0}\right)$ as $55 \%$. In the processing step, the mentioned raw data will be transformed by a specific rule and normalized to Processed Data of temperature ( $t^{\prime}$ ) and humidity ( $h$ '). The processed temperature data of 1,0 and 1 respectively means that the temperature outdoor is more that $15 \mathrm{Clower}$ than indoor, the temperature outdoor is the same as indoor, and the temperature outdoor is more that $15 \mathrm{C}$ higher than indoor. The processed humidity data of 0 and 1 respectively means that the indoor

\footnotetext{
${ }^{14}$ Rhinoceros. Retrieved on 29.06.2019 from https://www.rhino3d.com

${ }^{15}$ Grasshopper. Retrieved on 29.06.2019 from https://www.grasshopper3d.com

${ }^{16}$ Keyshot. Retrieved on 29.06.2019 from https://www.keyshot.com
} 
humidity is in the normal level (higher than 55\%), and the indoor humidity is too low (lower than $20 \%$ ) to be healthy.

\section{Mapping}

Next, the Processed Data is mapped to an initial Abstract Representation through a Mapping step. In this transformation, the dimensions of the Processed Data will be assigned to the scales of the variables used for the initial representations. Typically, those scales correspond to graphical primitives and graphical attributes. For SLS, the graphical primitives and the graphical attributes specifically refer to the light properties from light sources and the shape properties of physical objects.

The outcome of the data Mapping step make up the Abstract Representations which will be used to compose the presentation of SLS in the next step. In line with our SLS Framework, these Abstract Representations are described by embodying Active Lights and Active Objects with some specific attributes. However, these attributes are still abstract at this point as they are not yet fully defined.
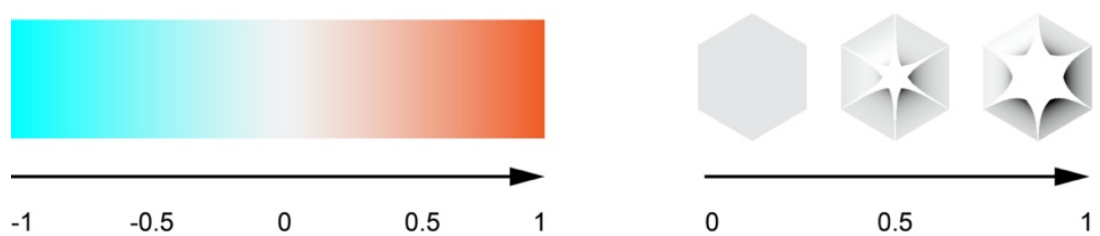

Figure 5.4. Mapping the Processed Data to the Abstract Representation of changes in light attributes and shape attributes: (Left) The colour mapping between the Processed Data of $\mathrm{t}^{\prime}$ (temperature difference between outdoor and indoor) and the changes of light attribute. (Right) The permeability mapping between the Processed Data of $h^{\prime}$ (relevant humidity indoor) and the changes of shape attribute.

In our example, we map the data of temperature change to the colour change of an Active Light source (see Figure 5.4 (left)). Meanwhile, the data of humidity change is mapped to the permeability change of an active surface (see Figure 5.4 (right)). The attributes of these representations are just partly defined. We only use a colour palette to the temperature data instead of an actual colour. Also, the permeability change is just a conception but without detailed descriptions, such as the technique of permeability change, the form of the surface, the density of the vent, etc.

\section{Combining}

This transformation step combines the Abstract Representations and turns them into a fully Specified Presentation that can be implemented. Considering the specificity of SLS, this 
transformation step is entirely dependent on the actual physical light-object interactions which will produce the specific light patterns. In this step, Preset Objects could be involved depending on the types of light patterns to be applied. For example, a preset surface is necessary to present projected light. So, the presentation of SLS is clearly different from conventional information visualisation systems that are displayed on a screen or presented using physical objects. The Combining step involves the following four operations:

Deployment. Firstly, the light sources and physical objects embodying the information will be deployed by following some specific types of light-object-interaction. Consequently, various light patterns will emerge simultaneously.

Materialization. Then, according to the chosen light patterns, the materials of the light sources and physical objects will be determined. For the physical objects, it means to determine a certain optical property, such as reflection, translucency, opacity, etc. For the light sources, it refers to selecting a particular type of light source, such as an individual point light source like a bulb, a composed light source like a matrix of LEDs, etc.

Specialization. At last, the variables of every Abstract Representation will be specified in a detailed definition. For example, applying a range of RGB values to the abstract palette colours for an Active Light source or modelling a specific shape-changing of an Active Object.

Decoration. This operation means to optimize the non-coding properties of the presentation to facilitate an aesthetically pleasing experience of reading the information presentation.

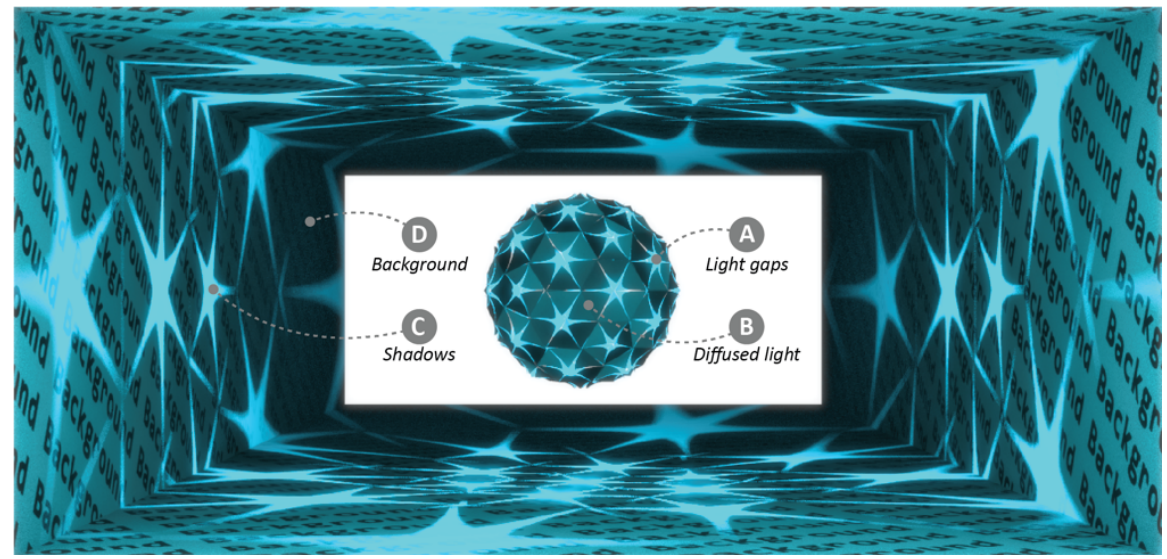

Figure 5.5. A simulation of the Specified Presentation in a computer implementation in our example. In this presentation, there are three kinds of light patterns: light gaps (point A), diffused light (point B), and shadows (point $\mathrm{C}$ ) that cast on the backgrounds (point $\mathrm{D}$ ). This presentation is realized by positioning the light source inside a deformable cover, which can change its permeability in shape. 
The outcome of this step is a Specified Presentation, which is an implementable scheme of combining the Abstract Representations. The Specified Presentation can be demonstrated in two ways: one is a simulation in a computer implementation, the other is a small-scale prototype in an experimental implementation.

In our example, the light representation (see the colour changes in Figure 5.4 (left)) and the physical representation (see the permeability changes in Figure 5.4 (right)) are combined as a shape-changing lamp (see Figure 5.5). It produces three kinds of perceivable light patterns, which are light gaps (see point A in Figure 5.5), diffused light (see point B in Figure 5.5), and shadows (see point $C$ in Figure 5.5) which will cast on another passive surface (see point $D$ in Figure 5.5). To make the shadow presentation as perceivable as possible, we propose to use a single RGB light bulb as the Active Light source which is covered by 240 pieces of star-shaped sheet. This sheet can change its permeability (see Figure 5.4 (right)) by opening/closing its translucent leaves.

\section{Rendering}

At last, the Rendering step makes the Specified Presentation perceivable by bringing it into existence in a real scene. Moreover, the outcome of this Rendering step is a Physical View which could be a lamp or a light installation. Such a Physical View portrays information in a decorative way, which is supposed to seamlessly merge into the user's context. For example, the shape-changing lamp (see Figure 5.5) could be either a ceiling lamp or a floor lamp. Several operations are involved in this transformation step:

Setting-up. This operation means to give the final Physical View a proper position to ensure that the user can observe it conveniently. In this operation, the pre-set SLS objects will be defined in a systematic way. For example, if the Physical View is a small-sized desk lamp, then the desk will be the pre-set object used to present the shadows.

Technical Realization. This operation concerns the technical parts of realizing the final Physical View. It includes the selecting of real light sources, actuators and sensors (if needed), and the programming. For example, the individual point light source could be an RGB LED or a Phillips Hue lamp, and the permeability change of this lamp could be realized by using a mechanical actuator or a pneumatized actuator.

Fabrication. This operation considers how to fabricate the components needed to implement the final Physical View. For example, the permeability change sheet in our example could be $3 \mathrm{D}$-printed or realised by laser cutting, depending on which shape-changing actuation technique is applied. 
In our example, the shape-changing lamp is finally transformed into a ceiling lamp (see Figure 5.6) placed in a living room, where it casts subtle shadows on the surroundings (ceiling, walls, paintings, etc.). The permeability change of the lampshade is realized by controlling the current that runs through different memory metal strips embedded in the translucent starshaped sheets. The light source is a Phillips Hue lamp which shines from bottom to top.

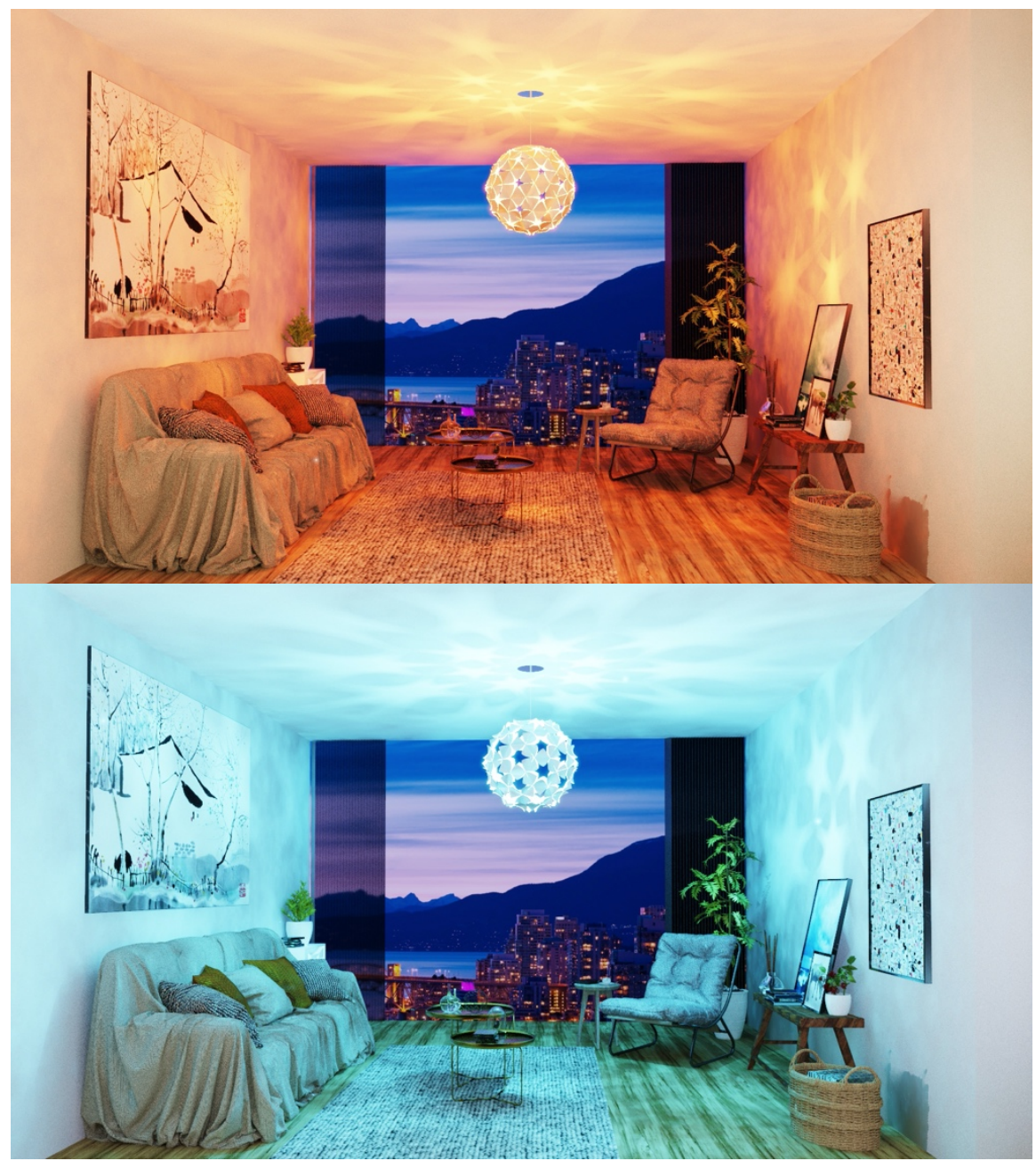

Figure 5.6. Two possible Physical Views in our hypothetical example: the top one indicates that the outdoor is warmer than indoor and the indoor humidity is a bit lower than normal state; the bottom one shows that the indoor is warmer than outdoor but too dry to be healthy. 


\subsubsection{Design alternatives for the information flow in SLS}

By our hypothetical example, we have demonstrated how to apply the information flow to describe a concept of SLS by explaining what options have been utilized for embodying the data transformation steps. In fact, there are many other available alternatives for modelling the information flow as well. For instance, it is possible to combine the Abstract Representations (see Figure 5.6) into an emitting luminous body (see Figure 5.7) by applying OLED technology as light source.

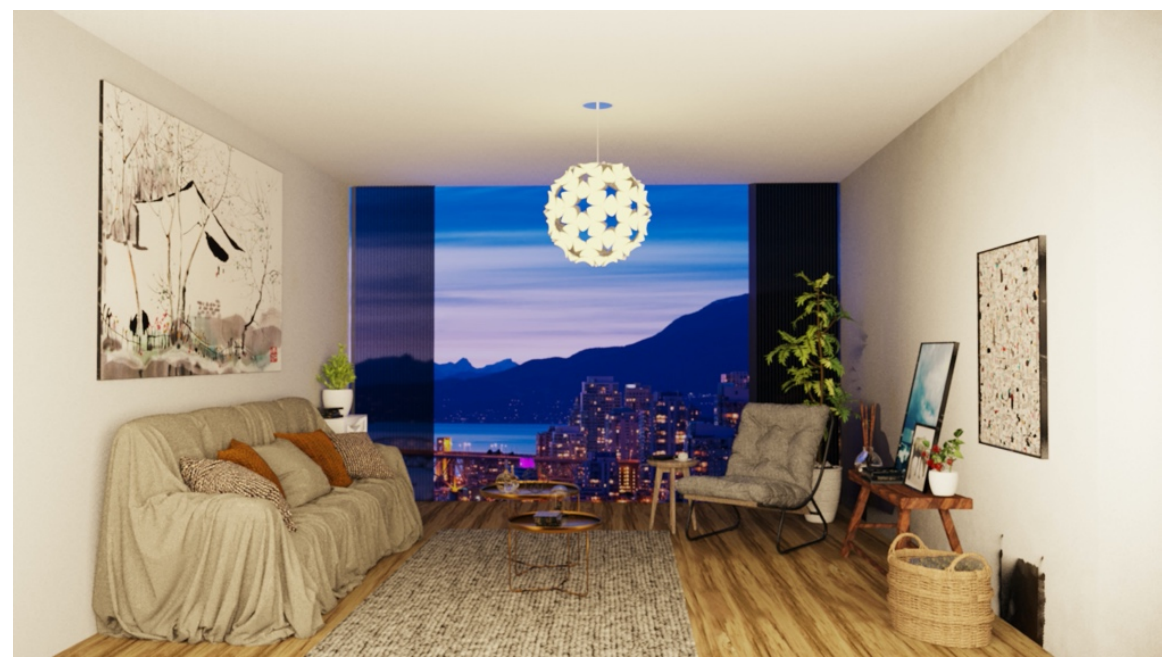

Figure 5.7. An alternative way of designing the shape-changing lamp in our hypothetical example. In this design, the Abstract Representations (see Figure 5.4) are combined into an emitting luminous body, in which the OLED panels are integrated with the star-shaped sheets.

Based on our SLS Framework, we present different design alternatives in the transformation steps of Mapping, Combining, and Rendering. These design alternatives provide designers with an overview of possible options that can be used for data transformation.

\section{Design alternatives for Mapping}

The main issue when designing SLS in the Mapping step is the linking of the specific parameters of data to selected attributes of SLS components. According to the SLS Framework, there are various parameters (section 3.3.2) that can be used in the Mapping of the Processed Data. The parameters of light attributes include on/off, intensity, colour, rhythm, and distribution. While, the parameters of shape-changing attributes include orientation, form, volume, texture, viscosity, spatiality, adding/subtracting, and permeability. 
The design alternatives mentioned above can be applied solely. For instance, in our hypothetical example, we only apply the light attribute of colour to represent the temperature information. Also, it is possible to integrate multiple alternative attributes into one particular representation. For instance, in our hypothetical example, the star-shaped sheet performs like the permeability changes in shape. This shape-changing is realized by manipulating the orientations of the leaves that composed the star-shaped sheet.

\section{Design alternatives for Combining}

The Combining step aims to draw a scheme of how the Abstract Representations will be combined into a Specified Presentation. The core of this scheme is applying the light-object interaction to produce light patterns. Based on the SLS Framework, there are six types of such interactions, which are: emitting, diffusing, blocking, reflecting, refracting, and projecting. They produce three different kinds of light pattern, which are initial light, sculpted light, and projected light. These light patterns have different characteristics for information presentation (see section 3.3.4).

In SLS, the light patterns can be simple if applying only one of the light-object interactions. For instance, Figure 5.7 shows a luminous body which using OLEDs to create emitting light patterns. Moreover, SLS can present rich light patterns by merging multiple types of lightobject interactions. For instance, in our hypothetical example, the shape-changing lamp can present three kinds of light patterns (see Figure 5.5), which are light gaps, diffused light, and shadows that cast on the surroundings.

\section{Design alternatives for Rendering}

Rendering, the final step in modelling the information flow, aims to put the Specified Presentation into the real world. The design alternatives for this transformation step are more about the technology realization parts. For the light sources, there are seven alternatives (see section 3.1.2), which are sunlight, ambient natural light, traditional light bulbs, LEDs, OLEDs, smart light bulbs, and beamer. For the shape-changing objects, the alternatives refer to the available techniques for shape-changing actuation. By now, there exist many compelling methods for shape-changing actuation (see Table 5.1), including electromagnetic, electromechanical, hydraulic, piezoelectric, pneumatic, smart materials.

In addition, the available materials and digital fabrication techniques are important for implementing SLS. The available materials refer to the optical materials (e.g., optical fibre, smart glass, etc.) to transform the light, the flexible materials (e.g., elastic fabric, silica gel, foam, etc.) to realise the shape-changing, and the conductive materials (e.g., conductive textile, conductive ink, etc.) to transmit signals and control light expressions or shape-changing. The 


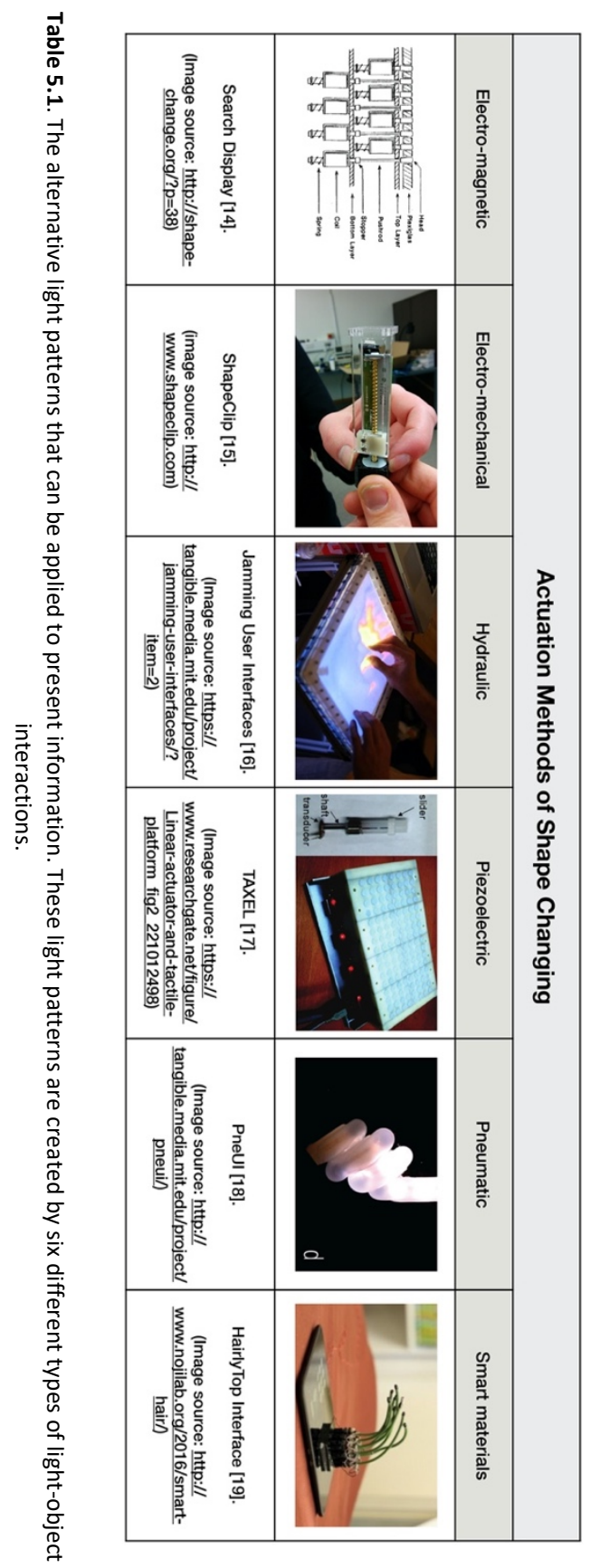


digital fabrication techniques are used to produce the mentioned materials into specific forms in a programmable way. In general, there are four alternative kinds of digital fabrication techniques, which are laser cutting, 3D printing, computer-numeric controlled (CNC) milling, printed circuit board (PCB) fabrication.

\subsection{Interaction Model}

The primary function of interactivity in visualisation systems is to allow users to dynamically alter the pipeline (also defined as information flow in SLS) to reveal different aspects of the data. Users can then integrate the various percepts and pieces of information over time to build a richer picture of the data and accumulate insights. As we have introduced in the previous section, there are four data transformation steps in the information flow of SLS. This naturally means that there are four different types of interactions, because the user could control the parameters in each step of the information flow regarding Processing, Mapping, Combining, and Rendering.

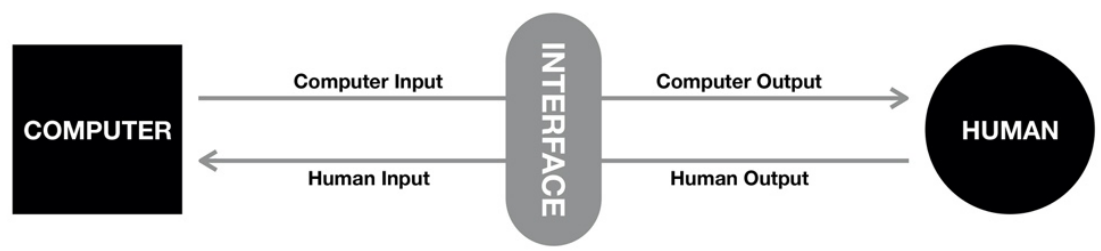

Figure 5.8. The basic model for human-computer interaction. The grey arrows are interactions between human and computer.

This Interaction Model (see Figure 5.9) is developed based on the basic model for humancomputer interaction (see Figure 5.8). In our case, the "computer" is the information transformation process, described in the previous section and represented by the black arrows in Figure 5.9. The Human Input is represented by the grey arrows in Figure 5.9, identifying four possible interaction loops that respectively effect the information flow through altering the four transformation steps (diamonds in Figure 5.9). In addition, our Interaction Model involves environmental factors as a trigger for interactions in SLS. By doing so, we expected to integrate SLS more seamlessly into the user's context. Therefore, the four means of interaction loop could be triggered either by the user directly or by the environmental or external aspects. 


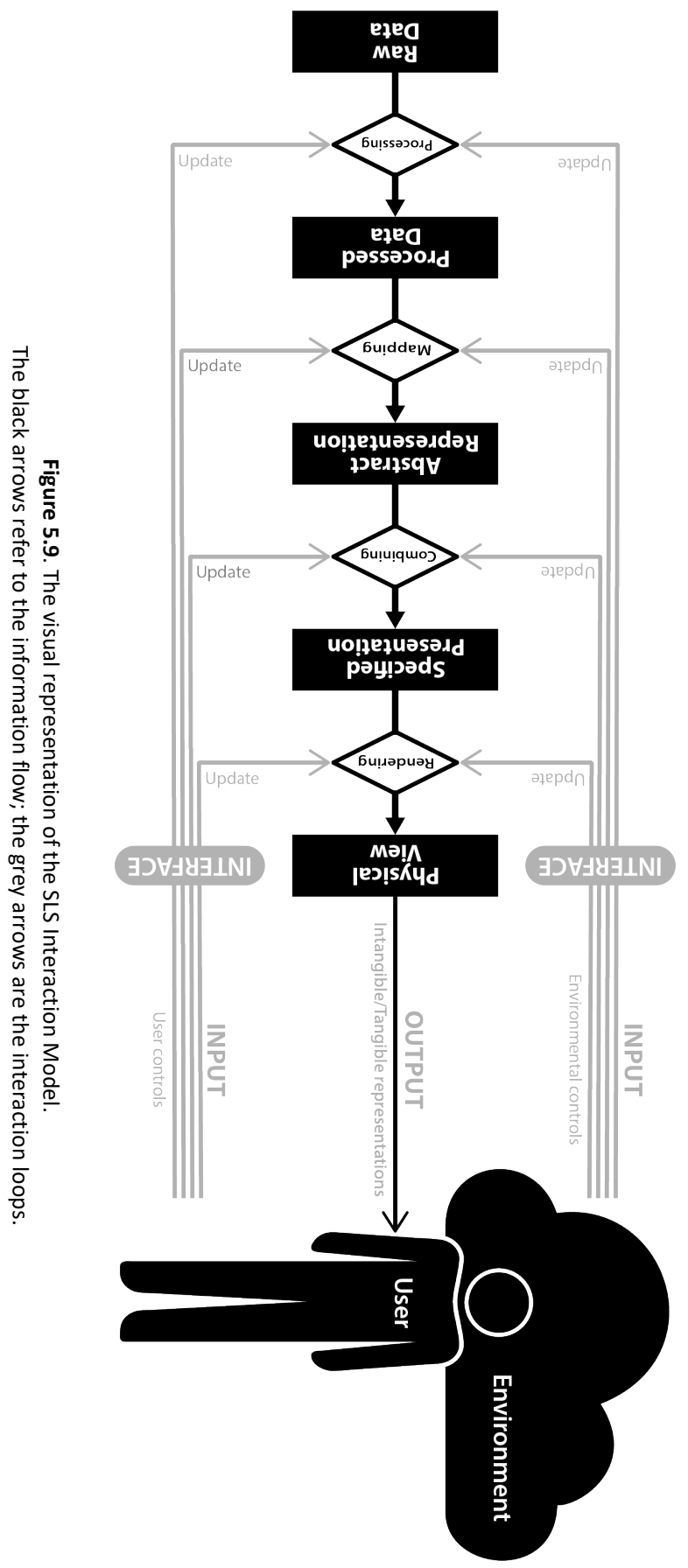




\subsubsection{Interaction loops}

In this section, we introduce the interaction loop, which directly alters the data transformation step of Processing, Mapping, Combining, or Rendering respectively without affecting other steps in the information flow. For example, if the user may want to know the temperature information in another city, then he/she can change the data Processing step by filtering the raw data related to that city. This implies that the user will perceive an updated Physical View as a consequence of the updated Processed Data, although the other transformation steps are unaltered.

\section{Interaction for Processing}

This interaction enables users to change the data processing to meet their demands of what information is needed. For instance, to increase the sample size of Processed Data for more detailed information, or to pick up an interested data category for information display. Also, the interaction for processing can enable the SLS to automatically alter the data processing according to the environment changes. For example, to adjust the data filtering based on the current location of the SLS, or to decrease the sample size of Processed Data when only a few audiences are appeared in the environment.

\section{Interaction for Mapping}

The purpose of this interaction is to meet the needs of modification towards the representation level, for example, to switch the style of information presentation. In our hypothetical example, the user can change the orange-to-blue light representation of the high-to-low temperature to yellow-to-green, according to his/her personal preferences (see Figure 5.10 (top)). The interaction for Mapping could be triggered by the environment impact as well. For example, a SLS system that can automatically increase its brightness when the environment light is too bright for users to recognize the light representation.

\section{Interaction for Combining}

The user interaction loop that changes the Combining step of the information flow aims to modify the style of information display on the presentation level. In this case, the recombination of Abstract Representations could be realized by applying a new way of deployment, materialization, specification, or decoration. As a result, a new form of Specified Presentation will be produced to portray information in another style. In our hypothetical example, the user can deploy the light patterns by reassembling the star-shaped sheets based on his/her need. For example, the bottom of Figure 5.10 presents a possible reassembled 
shape-changing lamp, which only keeps the meaningful shadows on the ceiling and parts of walls. While the area surrounding tea-tables becomes brighter for functional needs.

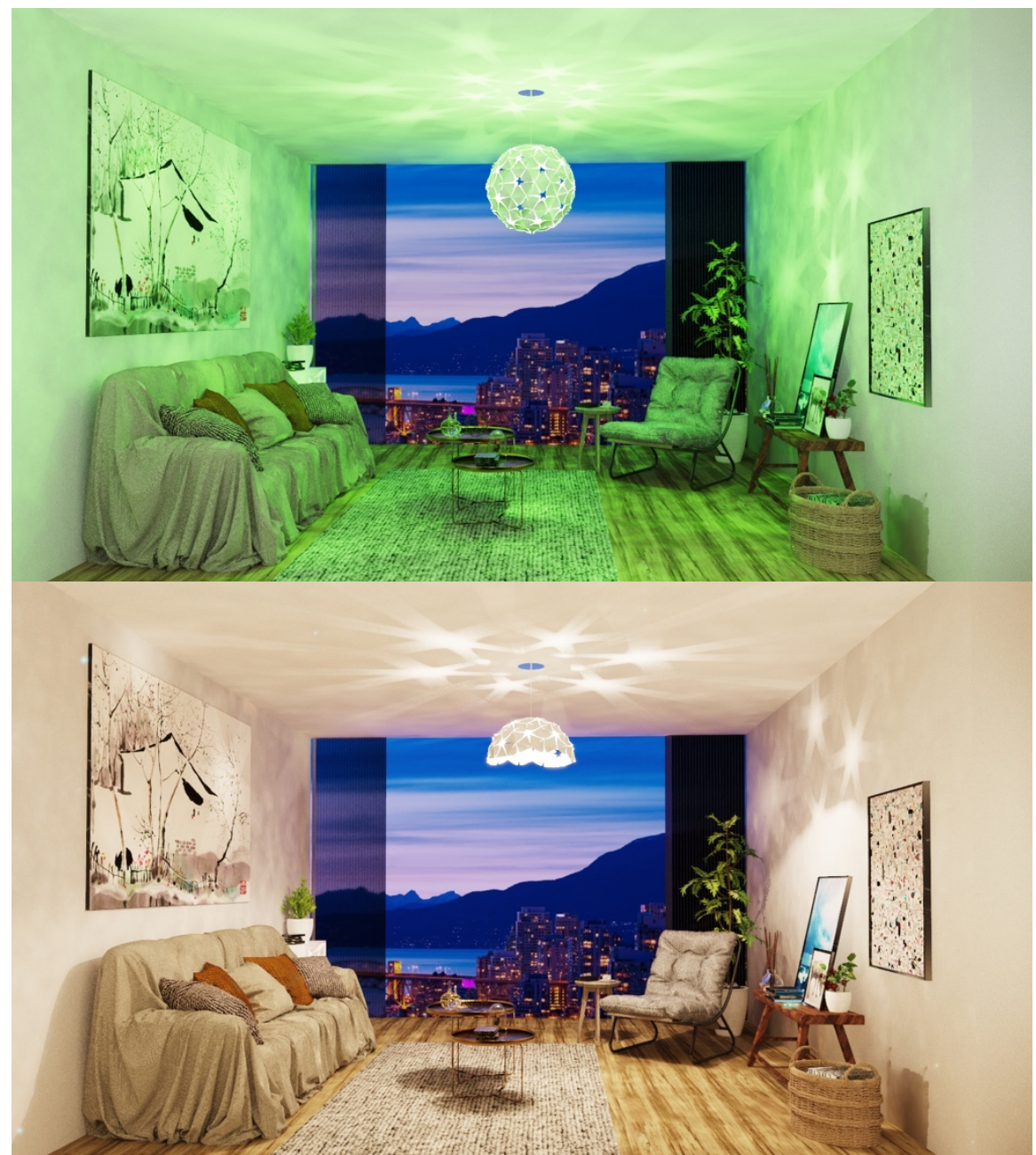

Figure 5.10. (Top) A possible interaction for Mapping in our hypothetical example. In this interaction, the user set the colour mapping for temperature as yellow-to-green instead of the default one (orange-toblue). (Bottom) A possible interaction for Combining in our hypothetical example. In this interaction, the user reassembles the shape-changing lamp to lighten the functional areas.

\section{Interaction for Rendering}

This interaction changes the final Physical View of SLS by altering the set-up of the Specified Presentation. However, the other steps of information flow do not change. In our hypothetical 
example, the user can reposition the shape-changing lamp in his/her drawing room according to his/her needs (see Figure 5.11). For example, moving the lamp from the ceiling to the floor to help the user perceiving the information more conveniently.

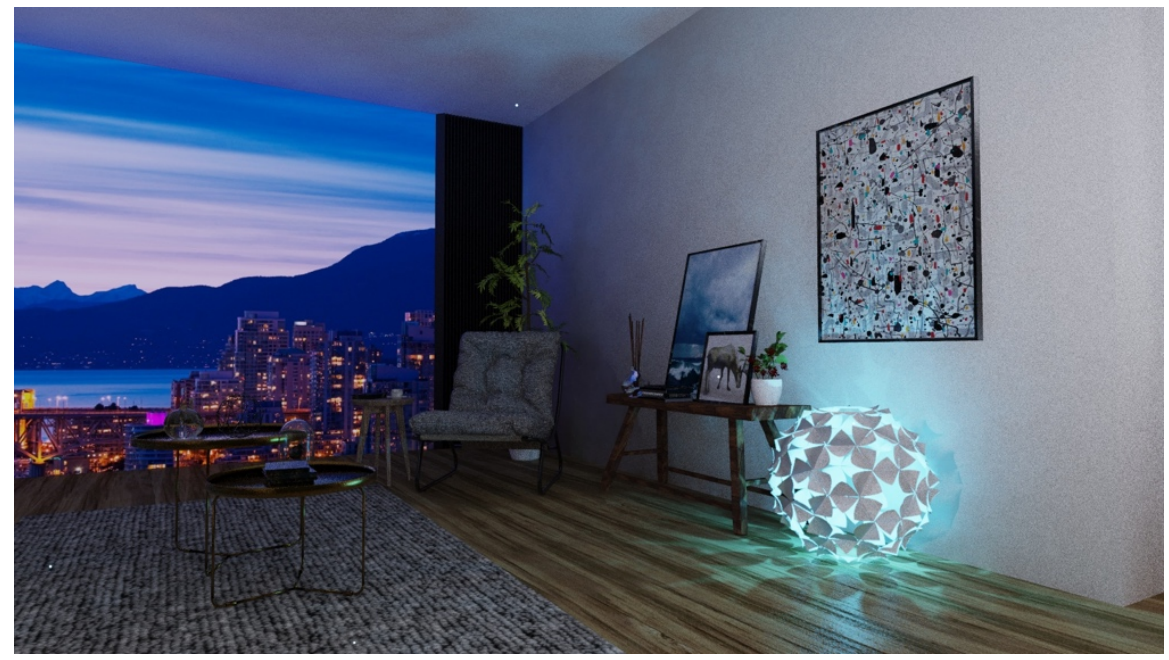

Figure 5.11. A demonstration of the interaction loop for altering Rendering in our hypothetical example. In this interaction, the user changes the form of the lamp based on his/her preference.

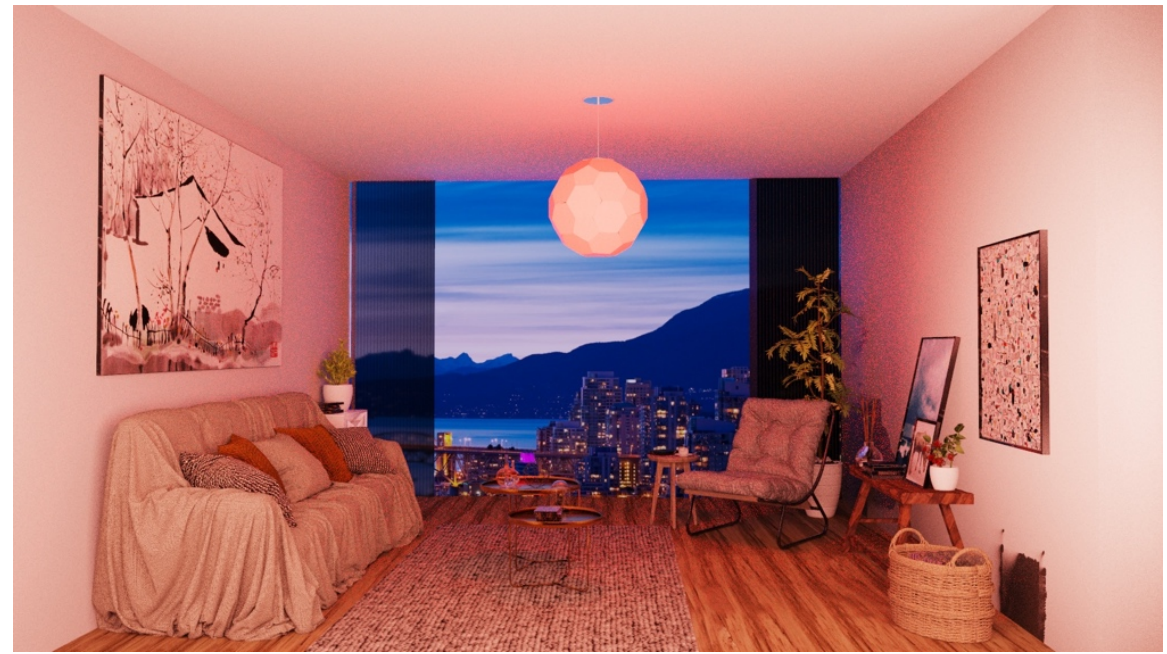

Figure 5.12. The interactions that alters both Mapping and Combining in our hypothetical example. In this interaction, the user shut down the displays of relevant indoor humidity.

In above, we have introduced the interaction loop that alters single data transformation step of Processing, Mapping, Combining, or Rendering. While these interactions could occur in a 
multiple way. In our hypothetical example, the user can turn off the representation of indoor humidity or outdoor temperature based on his/her needs. For example, when the user is absorbed into some primary task and does not want to perceive the shadows on his/her surroundings, he/she can shut down the representation of indoor humidity (see Figure 5.12). In this case, the lamp will only display the temperature information by means of light change. This interaction modifies both Mapping and Combining. For the modification of Mapping, the Processed Data related humidity will map to a constant permeability of the star-shaped sheets. For the modification of Combining, the lamp only produces diffused light without light gaps and shadows that cast on its surroundings.

\subsubsection{The alternative interaction techniques}

In our Interaction Model, we have presented four types of interaction loops within SLS. Basically, we have discussed the goals and effects of these interactions. Now we will discuss the alternative techniques to support these interactions. These techniques include two aspects, which are the possible controls that can trigger the interaction loops and the potential interfaces that can be applied to realize the interaction loops.

\section{The alternative controls}

The interaction loops can be triggered by either the user's explicit controls or implicit controls. The explicit controls are caused by the user who intentionally alters the information displays. For instance, the bottom of Figure 5.10 shows an interaction, in which the user explicitly turns off the representation of indoor humidity. The implicit controls are triggered by the user that unintentionally changes the information displays. For instance, the interaction shown in the bottom of Figure 5.10 could happen proactively if the system can sense that the user is absorbed into some primary task.

Also, the user's controls to trigger the interaction loops can be either on a local level or on a remote level. So far, information display systems almost exclusively focused on the local level. In this case, several taxonomies have been proposed, for example, Norbert's three interaction zones (Prante et al., 2003), Daniel's four interaction phases (Vogel \& Balakrishnan, 2005), and Greenberg's proxemic interaction (Diaz-Marino, Marquardt, Greenberg, Ballendat, \& Wang, 2011). These taxonomies provide alternative interaction techniques to support smooth transition from explicit interactions to implicit interactions. For example, the shape-changing lamp in our hypothetical example can adjust the eye-catching degree of information transfer by increasing the brightness when it detects users are approaching. For the interaction in the case of the remote level, we think that it is also an alternative interaction technique for the 
user's controls. For instance, in our hypothetical example, what if the user's relatives, who live in another city, can also access to the system online and turn on the representation of the indoor humidity when the user forgot to do that by himself/herself.

In addition, the interaction loops could be triggered by the environment impact. In this case, the system is enabled to automatically adapt the information displays to the dynamically changing environment. The environmental changes might be from the perspectives of climate or context. The climate refers to the light, sound, energy consumption, and even private security in the environment. For instance, in our hypothetical example, the shape-changing lamp can automatically increase its brightness when the environment light is too bright for users to recognize the light representation. The context changes refer to the activity, event, atmosphere, and even schedule of the user/users in the environment. For instance, in our hypothetical example, the shape-changing lamp can automatically increase the saturation of the light when there's a party in the drawing room.

\section{The alternative interfaces}

To transform the controls (either from the user side or from the environment side) mentioned above to the inputs for altering the information displays of SLS, the interfaces are essential to be considered when realizing the interaction loops. In general, the available alternatives include GUI (graphic user interfaces), TUI (tangible user interfaces) (Ishii \& Ullmer, 2012), and NUI (natural user interfaces) (Norman, 2010).

By means of a GUI, the user control can be input with an extra digital device like smartphone or tablet. For instance, in our hypothetical example, the interaction presented in the top of Figure 5.10 can be realized by a user controlling his/her smartphone with an App. In this case, the user can edit his/her preferred colours which represent the temperature information by means of light.

TUI merge the user control and Physical View of SLS into the same interaction space and in doing so provides additional haptic feedback when necessary. For instance, in our hypothetical example, the interaction presented in Figure 5.12 is carried out in a physical way by allowing the user to manually move the lamp from the ceiling to the floor where he/she preferred to perceive the information displays. Moreover, this interaction can be improved by involving a tangible interaction: when the user places the lamp far away from his/her position, the lamp automatically increases its brightness to make sure the information that is displayed is always clearly visible; when the user places the lamp close to his/her position, the lamp automatically decreases its brightness to avoid obtrusiveness. 
The example mentioned above shows a tangible interaction that integrates TUI into the SLS components. Besides, it's also possible to employ the environmental objects as the extra individual tangible interfaces. For instance, in our hypothetical example, what if the representation of the outdoor temperature can automatically be turned off when the user picks up a particular book and starts reading. In this case, the tangible interface could be a smart bookmark in this particular book, which needs concentration to read without distraction from the light changing in colour.

NUI enable the user to control SLS in a natural way without using artificial control devices but by sensing the user's activities (e.g., Proxemic Interaction (Diaz-Marino et al., 2011)), movements (Gesture Interaction (Wagner, Malisz, \& Kopp, 2014)), and voice (Speech Interaction (Wagner et al., 2014)). For instance, in our hypothetical example, the interaction presented in Figure 5.12 enables the user to turn on/off the representation of either the indoor humidity or the outdoor temperature based on his/her needs. This interaction could be triggered by detecting the user's voice commands such as, "stop displaying the humidity information", "what does the outdoor temperature feels like?", and "do I need to turn on the humidifier?"

\subsection{Using the Interaction Model}

In the previous sections, we introduced our Interaction Model and discussed design alternatives. To validate our Interaction Model, we analyse its usefulness by envisioning an alternative design of Timer Lamp (see Figure 5.13). Timer Lamp is our first design case of SLS developed at the start of the project (see section 2.2). We now present how we can use the Interaction Model to support a systematic approach to design a concept like Timer Lamp.
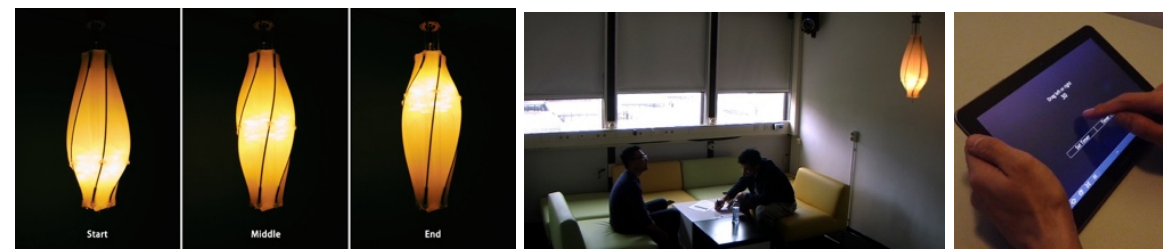

Figure 5.13. (Left) The information displays of Timer Lamp during a meeting; (Middle) Scenario of Timer Lamp; (Right) Interaction of setting time by using an App on a tablet.

In this section, we first briefly describe the previous concept idea of Timer Lamp by illustrating its information flow and interaction loop. Based on that, we propose new design possibilities for Timer Lamp by uncovering the ignored design alternatives and interaction loops. Then, we combine these design possibilities into three new design concepts of Timer Lamp. By 
comparing these new design concepts according to four initial criterions, we finally generate a preferred design concept for Timer Lamp which is arguably better. Based on this analysis, we also discuss the limitations of our Interaction Model during the design concept generation.

\subsubsection{Describing the existing design concept}

In the previous study, we described the concepts of our design cases in a general way. For example, in the case of Timer Lamp, we described it like: Timer Lamp is a ceiling lamp that changes the vertical position of its interior light sources and the form of its exterior lamp body to portray the progress of an informal meeting. It also enables user to set the duration of the meeting on a tablet.

The Interaction Model can help designers to envision their design concepts in a more systematic way. Taking the example of Timer Lamp, we first describe its design concept with an illustration in Figure 5.14. Afterwards, we specify the information flow and interaction loop in the concept of Timer Lamp.

The Raw Data of Timer Lamp is real time clock data (for example: 14:15 pm). It might be filtered for a certain period (for example from 14:00 pm to 15:00 pm) depending on the user's input, which is controlled with a tablet interface, and compiled into Processed Data, in this case real numbers ranged from 0 to 1 . Each Processed Data entry is mapped to a corresponding Abstract Representation of either the vertical position of the LEDs or the form of the lamp body. These two types of Abstract Representation are combined into a luminous body (Specified Presentation), in which the initial light is diffused by the shape-changing lamp shade. Finally, this luminous body is implemented as a ceiling lamp (Physical View) with a fixed position in the meeting room.

In this illustration, we identified what the Raw Data, Processed Data, Abstract Representations, Specified Presentation and Physical View exactly are. We also indicated in which step the interactivity is engaged by specifying the type of interaction technique. These descriptions definitely help us to understand our design concept in a more systematic way. Further, we expect these systematic descriptions as a starting point for the further design of Timer Lamp in terms of discovering the unexplored possible concepts. 


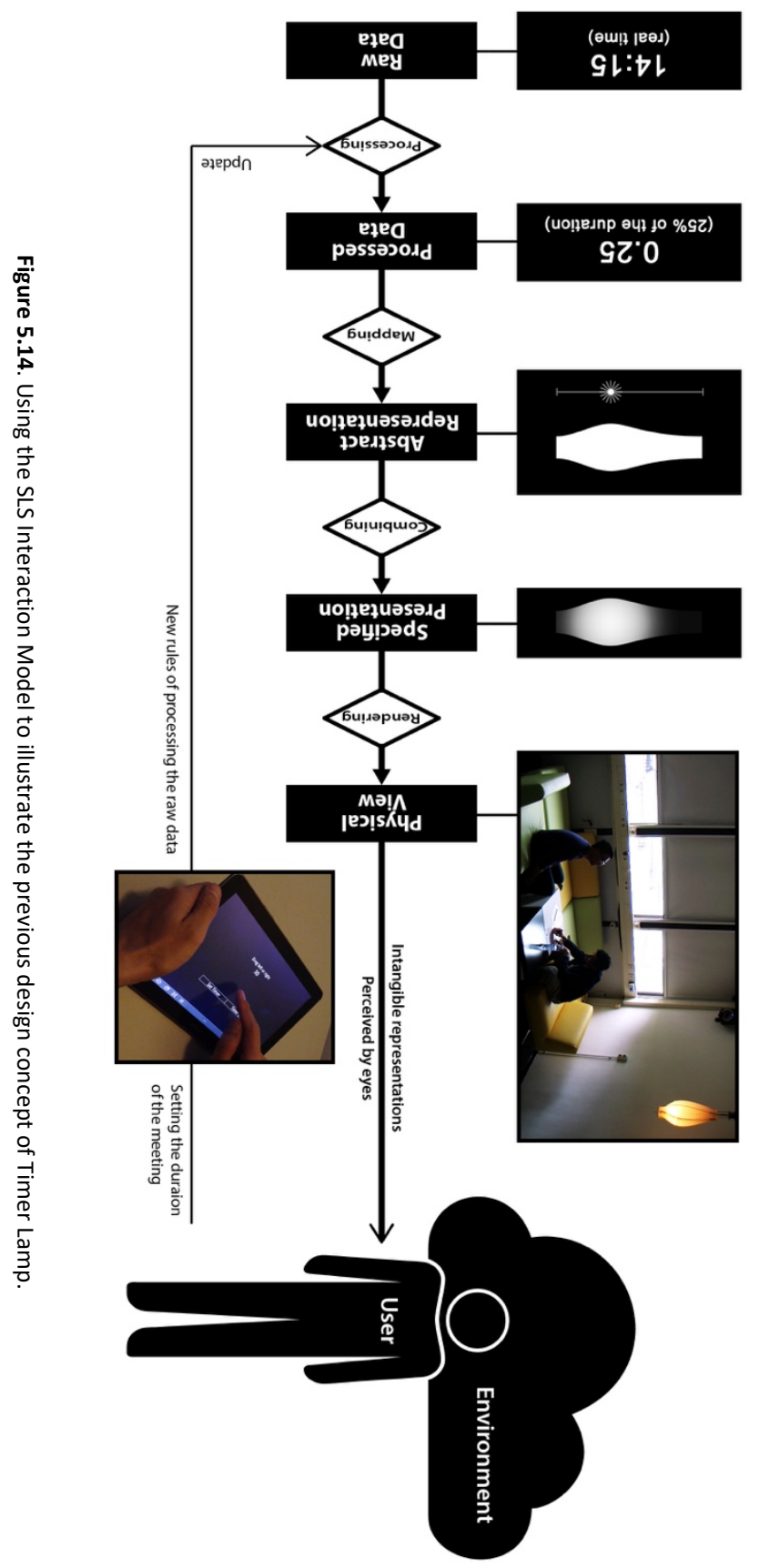




\subsubsection{Discovering new design possibilities}

By using our Interaction Model to illustrate the concept of Timer Lamp, we easily get an overview of which design options are used and which are missed. This overview naturally stimulates us to discover new possibilities by considering the unexplored design options. For example, what if we would use an alternative information flow, and what about applying new interaction loops with new interaction techniques? In this way, we can come up with some new ideas in the following two steps: First from the perspective of data transformation and then from the perspective of interactivity.

The first step follows the information flow of our Interaction Model. We review other alternatives in terms of Processed Data, Abstract Representation, Specified Presentation, and Physical View. Soon, we found out some potential alternatives which also might be applied to design Timer Lamp. All of these potential alternatives are selected with reasons.

\section{Alternative ideas for processing}

For the initial design concept of Timer Lamp, the Raw Data are processed into a linear sequence $t$ (see Figure 5.15 (left)). But what about a non-linear mapping? For example, the processed sequence could grow faster than the real time (see Figure 5.15(right)). This type of Processed Data could cause the information presentation to become more and more conspicuous when getting closer to the end state of the meeting. In this way, we think that the presentation might enhance the functionality of Timer Lamp at the right moment when the user is really willing to gain information.
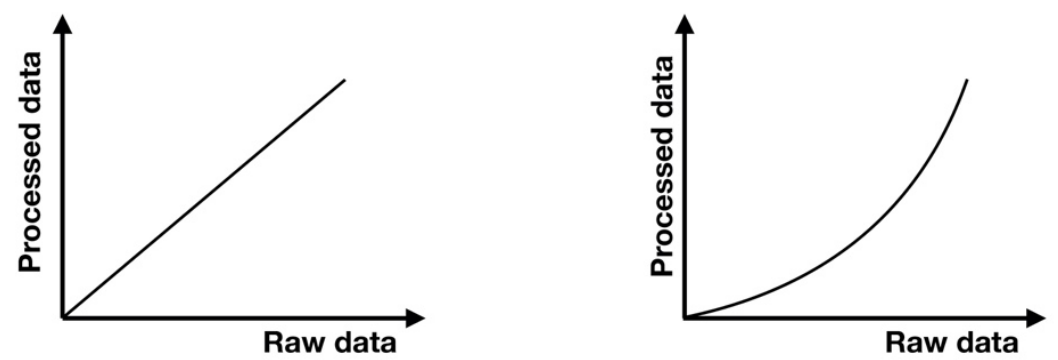

Figure 5.15. (Left) The original way of data processing for Timer Lamp. (Right) A new concept of data processing for Timer Lamp.

\section{Alternative ideas for Mapping}

There are other Abstract Representations that can inspire us with new meaningful representations of time. For example, changing the light intensity in a certain rhythm can 
represent some important moment during the meeting, the light colour changes from blue to red can represent the metaphor of the meeting's atmosphere from a relaxed mood at the beginning to a more strained atmosphere towards the end of the meeting. The shape change of volume can indicate that the remaining time of the meeting is decreased, and the permeability change of physical shape can represent the conversational state in the meeting from open to more directive.

\section{Alternative ideas for Combining}

By combining these potential Abstract Representations, we see different types of Specified Presentations of time information. These Specified Presentations are presented with different light patterns. For example, the combination of the permeability changes of shape and the colour change of light could produce either colour changing light gaps or shadows on an additive Preset Object. Alternatively, we can also combine the volume change of shape and the colour change of light to produce a volume changing luminous body. In addition, both of these two types of Specified Presentations are able to notify some important moment during the meeting by changing the light intensity in a certain rhythm.

\section{Alternative ideas for Rendering}

Before bringing these Specified Presentations mentioned above into reality, we think about how these Specified Presentations are supposed to be presented in different forms of lighting. The colour-changing light gaps can be presented as a lamp that is either fixed on the wall or placed on a table. At the same time, either the wall or the table could be applied as Preset Object to present the colour changing shadows. Also, the volume changing luminous body could be either a drop-like lamp that hangs on the ceiling or a balloon-like lamp that floats in the air.

The second step follows the interaction loops to discover the possibilities of designing Timer Lamp concerning the interactivity aspect. In this step, we investigate which interaction loop could be possibly integrated into the information flow of the alternative design concepts we envisioned. Also, we can select proper interaction techniques for the possible interaction loops. Our initial design concept of Timer Lamp applies the interaction loop to enable users to alter the processing step of the information flow by means of a tablet interface (see Figure 5.13 (right)). Below, we uncover other possibilities of integrating interactivity in the four transformation steps of Timer Lamp.

\section{Alternative Interactions for processing}

Firstly, interaction in the processing step means to process real 'time' into a sequence of numbers, which represents the duration of the meeting for users. Besides using a tablet interface, we can also employ tangible interaction as an interaction means. For example, the 
user can spin the physical form of Timer Lamp to set the meeting time. But what if the processing step could also be modified by the environment? For example, the Timer Lamp can sense the user's emotion in the discussion and automatically shorten the meeting when the tension in the meeting is heightened.

\section{Alternative interactions for Mapping}

Secondly, thinking about the interactivity in the Mapping step, what if the user can customize which two colours that he or she prefers to represent the start and end point of the meeting? One reason to change this colour mapping is that the meaning of colour will be different according to different cultural backgrounds. In addition, by letting the users choose their own colour mapping can protect their privacy if the meeting room is transparent to the other people. Such interactions can be realized through either a conventional graphic interface (e.g., a tablet) or a tangible interface (e.g., a Rubik's cube). Also, Timer Lamp could measure the environmental light conditions and then automatically adjust its brightness to make sure the information that is displayed is always clearly visible.

\section{Alternative interactions for Combining}

Thirdly, for the interaction loop in the Combining step, the user can decide which light pattern he or she wants to see. For example, the user can move the light sources a little bit further from Preset Objects to blur the shadows if they consider the shadows being too distracting for them. There are several alternative interaction techniques that can be applied, such as manipulating the lamp by touch or gesture, and interacting with an additional tangible interface by hand. Also, Timer Lamp could automatically decide whether the shadows should be presented or not by sensing the user's implicit activities, which is an important environmental factor. If the shadows would be presented on the table, it would be smart for the Timer Lamp to fade these shadows when the user needs to write or read something at the table.

\section{Alternative interactions for Rendering}

Finally, we think that either the user or the environment can modify the Physical View of Timer Lamp to make it appropriately present its Specified Presentations. For example, Timer Lamp can be portable which enables users to place it to the desired position they want.

\subsubsection{Envisioning new design concepts}

Our Interaction Model helps to discover many alternative possibilities for designing Timer Lamp. But these design possibilities are only segments that are separated in different parts of our Interaction Model. It is still difficult to envision how a new Timer Lamp would look like. 
To come up with complete design concepts, we integrate the discovered design possibilities into three different scenarios, leading to three new design concepts for Timer Lamp.

\section{Concept 1: adapting to environmental light}

This design concept aims to provide an adaptive information representation according to the environmental light condition. This design concept is specified in Table 5.2. Also, we illustrate the interaction loops in this design concept in Figure 5.16.

\section{Processing.}

The Raw Data is still the real 'time' information, which is processed into an arithmetic sequence that corresponds to the duration of the meeting. This is manipulated by the user with an App on a tablet.

\section{Mapping.}

The sequence of numbers are the Processed Data, which will be mapped to two types of Abstract Representation. One is colour change of light from blue to red. The other is the volume change of shape from full to empty. The brightness of Timer Lamp is automatically adapted to the dynamic environmental light. For example, when the environment light is turning brighter, Timer Lamp will also subtly turn brighter to make sure the information representation can be perceived clearly.

\section{Combining.}

These two Abstract Representations are combined as a luminous body, where the light is diffused by a balloon-shape shade.

\section{Rendering.}

This luminous balloon is fixed with a string which is attached to a moveable base. In this way, the user can move Timer Lamp anywhere in the meeting room according to his or her demand.

Table 5.2. The details of each data transformation step in the Concept 1 of Timer Lamp. 


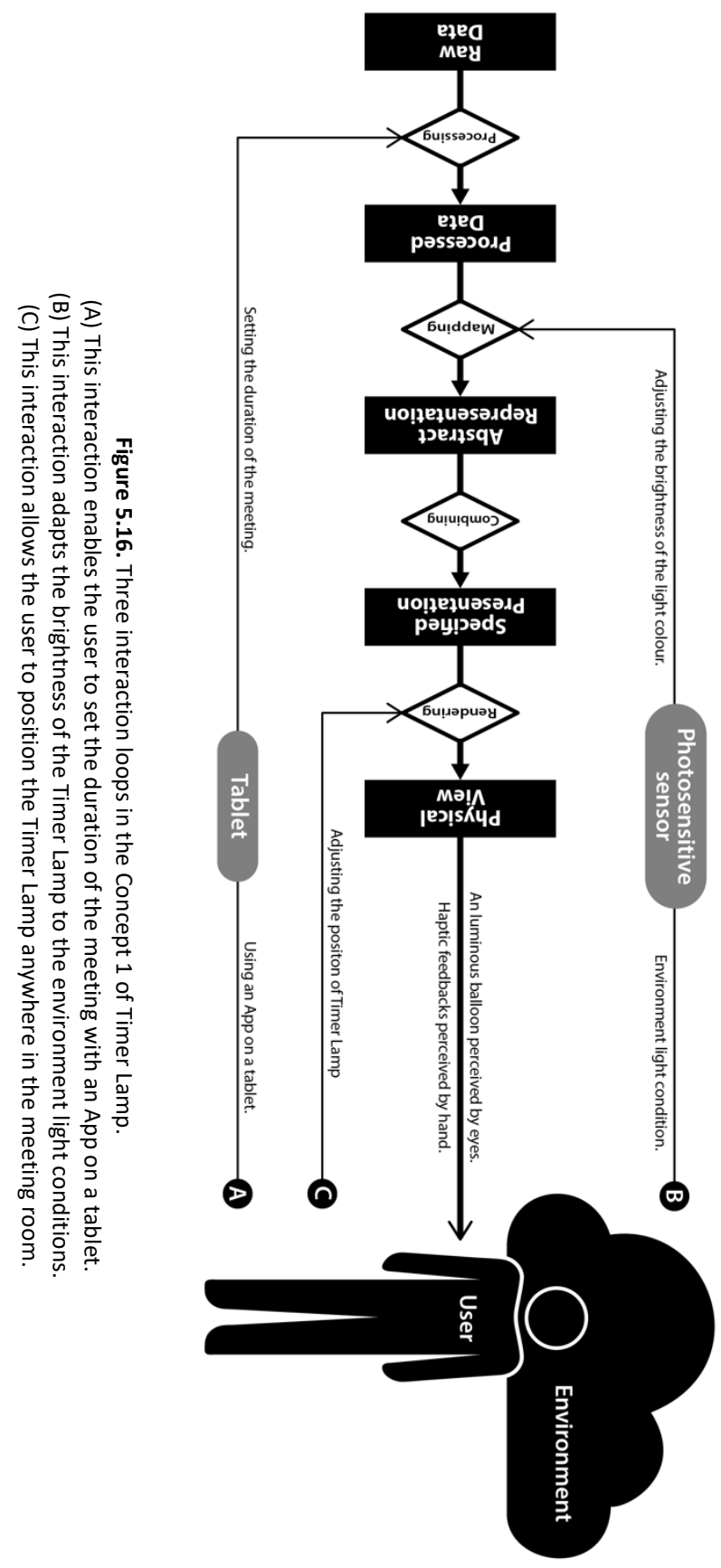




\section{Concept 2: modifying light patterns}

This design concept aims to remind the user to notice the progression of time at particular moments. These particular moments are set by the user and depend on his or her personal preference. This design concept is specified in Table 5.3. Also, we illustrate the interaction loops in this design concept in Figure 5.17.

\section{Processing.}

The Raw Data is still the real 'time' information, which is processed into an arithmetic sequence that corresponds to the duration of the meeting. The user can set the duration of the meeting by squashing the lamp body. This control provides users haptic feedback, i.e., they feel a "click," when they squash the lamp to forms corresponding to 20-minute, 30minute, 40-minute, 50-minute, or one hour, respectively.

\section{Mapping.}

The sequence of numbers are the Processed Data, which will be mapped to two types of Abstract Representation. One is intensity change of light from dark to bright. The other is the form change of shape from squashed state to initial state.

\section{Combining.}

These two Abstract Representations are combined as a luminous body, where the light is diffused by a fusiform shade. Also, this luminous body produces two more types of light pattern. One is light gaps, which are perceived on the lampshade. The other is shadows, which are cast on the table. The user can modify these two types of light pattern by spinning the two layers of the lampshade based on his/her interests on perceiving the light gaps and shadows.

\section{Rendering.}

This luminous fusiform is placed on the table in the meeting room.

Table 5.3. The details of each data transformation step in the Concept 2 of Timer Lamp. 


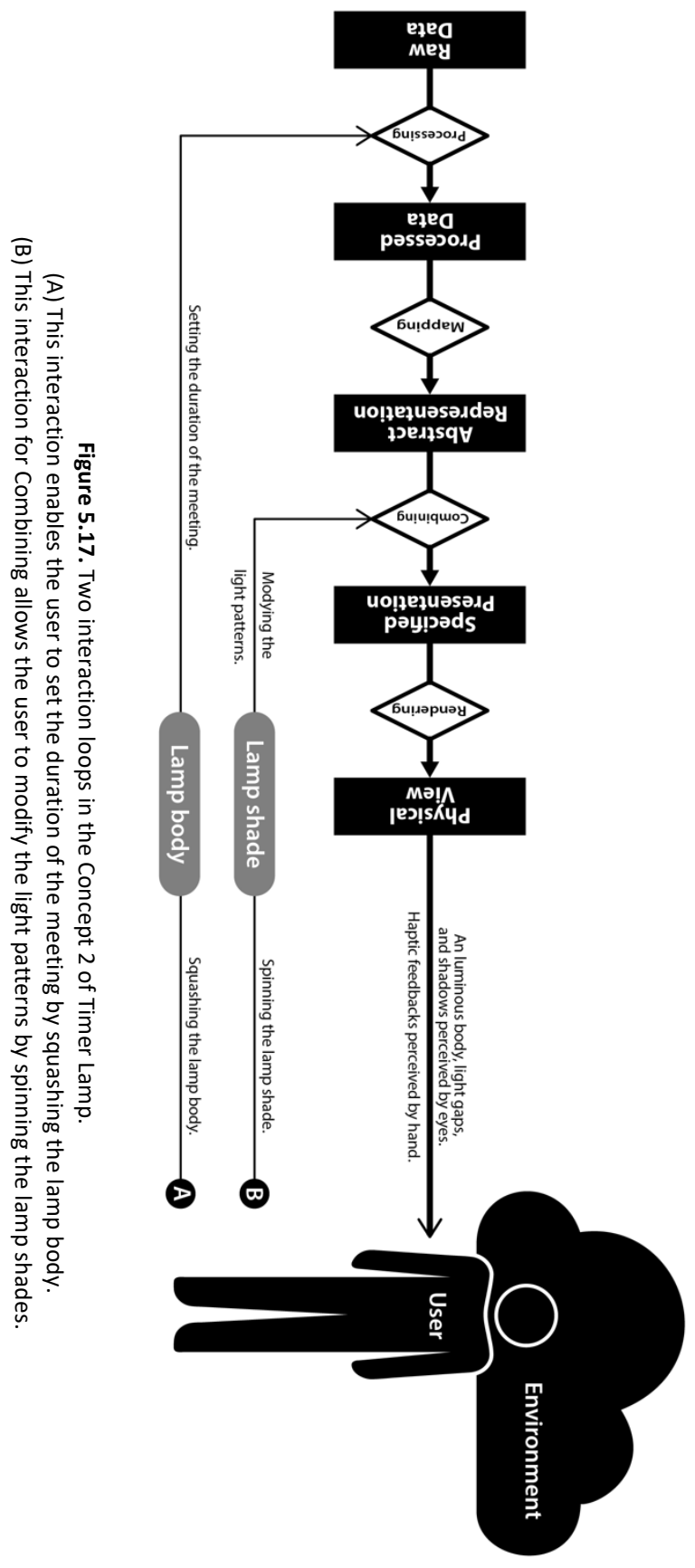




\section{Concept 3: notifying at particular moments}

This design concept aims to enable Timer Lamp to automatically manage the meeting progress by sensing its environmental atmosphere. This design concept is specified in Table 5.4. Also, we illustrate the interaction loops in this design concept in Figure 5.18.

\section{Processing.}

The Raw Data is still the real time information, which is processed into a sequence of increasing numbers but not in an arithmetic manner. This processing step depends on two variables: one is the length of the sequence that corresponds to the duration of the meeting, the other is the smoothness of the sequence that corresponds to the schedule of the meeting. Both of these variables are manipulated by the user with a dedicated App on his or her smartphone.

\section{Mapping.}

There are three types of Abstract Representations that need to be mapped to the Processed Data. The first one is the colour change of light from 'colour $\alpha$ ' to 'colour $\beta$ ', which maps to the regular Processed Data from the start to the end. The 'colour $\alpha$ ' and 'colour $\beta$ ' are defined by the user with the dedicated App on his or her smartphone. The second one is the volume change of physical shape from full to empty, which also maps to the regular Processed Data from the start to the end. The third one is the intensity change of light, which maps to some particular parts of the Processed Data. This means that Timer Lamp will subtly blink at particular moments in time. The particular moments can be defined when the user was setting schedule of the meeting.

\section{Combining.}

The Abstract Representations are combined into a luminous body, where the light is diffused by a fusiform shade.

\section{Rendering.}

This luminous fusiform is fixed on the ceiling of the meeting room.

Table 5.4. The details of each data transformation step in the Concept 3 of Timer Lamp. 

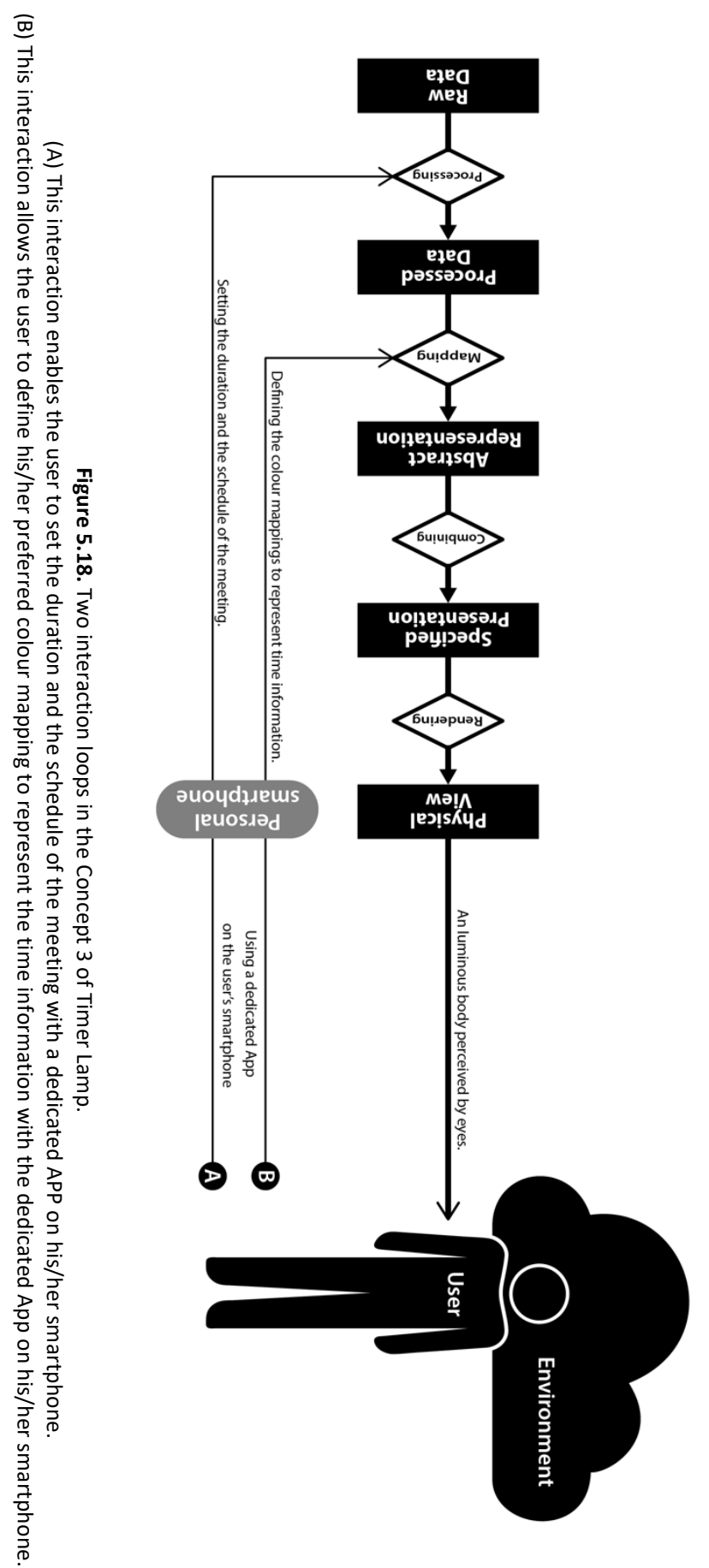


\subsubsection{Criteria for generating a design concept}

In the above section, we have introduced how our Interaction Model can help us to systematically describe and further discover new possibilities for designing Timer Lamp. In this way, we developed and proposed three new design concepts of Timer Lamp. However, it is impractical to directly implement all of these concept ideas without any reasoning. To generate the most promising design concept, a selection, combination or integration of these new design concepts is necessary. This integration can be carried out by first selecting the promising aspects of each design concept and then combining these promising aspects into one final concept.

To select the promising aspects in each concept idea, an evaluation process is needed to compare the relative strengths and weaknesses of these different design concepts. Many criteria could be involved in this evaluation process with respect to user, client, technology, material, time, budget, and even designer's motivation. Also, there are many different methods for evaluating the concept ideas based on these criteria, such as learning from the existing experience, voting in a team of designers and clients, testing a low-fidelity prototype with end-users, and so on.

But whatever evaluation methods and criteria the designer use, we believe our Interaction Model can always contribute a systematic structure for the evaluation process and its outcomes. According to the definition of SLS (see section 3.2.5), there are four general criteria that are particularly important for a good SLS design concept. They include subtleness, unobtrusiveness, aesthetics, and environmental appropriateness. In the context of Timer Lamp, we specify these four criteria as below:

- Subtleness. The information (re)presentation and interactions of Timer Lamp should be subtle without demanding focused attention from the user when he/she is in a meeting.

- Unobtrusiveness. The information (re)presentation and interactions of Timer Lamp should be non-intrusive but provides an appropriate level of notification according to the different importance of the time information.

- Aesthetics. The information (re)presentation of Timer Lamp should be aesthetically pleasing rather than being useful only.

- Environmental appropriateness. The information (re)presentation of Timer Lamp should seamlessly merge with the environment. Also, the interactions of Timer Lamp should be natural, which can be appropriately integrated into the context. 
Next, we will show how we integrate the three new design concepts of Timer Lamp into a final design concept. We firstly select the promising ideas by evaluating the capacity of the three new design concepts in each of the four transformation steps. Every design option is compared in terms of our four criteria. After this evaluation, we combine the advantages of each design concept to arrive at the most promising concept to be developed further in the future. The most promising design concept is illustrated in Figure 5.18 and Table 5.5.

\section{Comparing the design concepts to select the promising ideas}

In the processing step, Raw Data is transformed in two different ways. One is only setting the duration of the meeting by the tangible manipulation of spinning the lamp body (see the concept 2 in section 5.4.3). The other is setting both the duration and the schedule of the meeting (see the concept 1 in section 5.4.3 and the concept 3 in section 5.4.3). We choose the tangible manipulation because it is more intuitive compared to using an additional digital device. For example, the user can naturally touch the pre-set marks on the lampshade, which may correspond to different types of schedule making. Moreover, these marks could be some additional aesthetics elements of Timer Lamp.

In the Mapping step, we select four types of Abstract Representation. On the one hand, the intensity-change of the light (see concept 2 in section 5.4.3) represents regular time information most often, while the colour-change of the light (see concept 3 in section 5.4.3) represents the particular moments during the meeting. That is because we were somehow concerned that the colour change of light might interrupt the user's main task. However, using colour change of light to represent a particular moment is a subtle but efficient information notification. On the other hand, the form-change of the shape (see concept 2 in section 5.4.3) will be used because it naturally merges the user input with the system output, which can make it easier for the user to learn, understand, and remember. Moreover, it produces rich light patterns which can be more aesthetically pleasing than using the Abstract Representation of volume-change in shape (see concept 1 in section 5.4.3).

We select all the three mentioned light patterns, which are the diffused light, light gaps, and shadows. However, we decided to apply them in different scenarios. For the diffused light, it is used when the user needs a calm light atmosphere on the area of Preset Objects (e.g., the table used for either showing the shadows). For example, when the user is going to write something on the table, the light gaps will be sealed and only diffused light will be used for information presentation, which is less intrusive than light gaps and shadows. For the light gaps and shadows, they are employed when the user is open to have a florid light atmosphere around. For example, when the users are talking to each other, the light gaps and shadows can be aesthetically located in the periphery of the user's attention without interruption. The 
interaction that is necessary to modify the Combining step will be realized implicitly by sensing the user's activity during the meeting. This interaction can integrate Timer Lamp in the user's context more seamlessly than just allowing the user to explicitly modify the light patterns (see concept 2 in section 5.4.3).

Finally, we enable the user to move Timer Lamp on a table as he or she desires to do so (see concept 1 in section 5.4.3). While, considering the instability of the environment light condition, we also enable the Timer Lamp to adjust its brightness according to the brightness of the environmental light (see concept 1 in section 5.4.3). This interaction actually alters the Mapping step of Timer Lamp. These two choices aim to make Timer Lamp better integrate in the user's context.

\section{Combining the selected ideas to proposing the final concept}

Finally, we propose the final concept of Timer Lamp by combining the selected ideas mentioned above. This final design concept is specified in Table 5.5. The active interaction loops in this design concept are illustrated in Figure 5.18.

\section{Processing.}

The Raw Data is the real time information, which is processed into a sequence of increasing numbers but not in an arithmetic manner. This processing step depends on two variables. One is the length of the sequence which corresponds to the duration of the meeting. The duration of the meeting is set by the user by squashing the lamp body. This control provides users haptic feedback, i.e., they feel a "click," when they squash the lamp to forms corresponding to 20-minute, 30-minute, 40-minute, 50-minute, or one hour, respectively. The other variable of the processing step is the smoothness of the sequence which corresponds to the schedule of the meeting. This is set by the user by touching the marks on the lamp shade. This interaction can trigger different types of notification in different timings during the meeting such as: the middle of the meeting, quarter points of the meeting, or end of the meeting in 5 minutes.

\section{Mapping.}

There are three types of Abstract Representations that need to be mapped to the Processed Data. The intensity-change of the light and the form-change of the shape represent regular time information all the time. The brightness of light maps to some particular parts of the Processed Data. This means that Timer Lamp will subtly blink at particular moments in time. The particular moments can be defined when the user was setting schedule of the 
meeting. Moreover, the brightness of Timer Lamp is automatically adapted to the dynamic environmental light. For example, when the environment light is turning brighter, Timer Lamp will also subtly turn brighter to make sure the information representation can be perceived clearly.

\section{Combining.}

These Abstract Representations mentioned above are combined as a luminous body, where the light is diffused by two fusiform shades. By spinning these two shades, this luminous body can also produce two more types of light pattern. One is light gaps, which are perceived on the lampshade. The other is shadows, which are cast on the table. This interaction is triggered by Timer Lamp itself by sensing the user's activity. For example, when the user is going to write something on the table, the light gaps will be sealed to erase the shadows on the table. While, when the users are talking to each other, the light gaps and shadows can subtly emerge themselves in the periphery of the user's attention to provide an aesthetic light atmosphere.

\section{Rendering.}

This luminous fusiform body is placed on the table in the meeting room. While, the user can move it to anywhere in anytime on the table according to his or her demand.

Table 5.5. The details of each data transformation step in the final concept of Timer Lamp.

\subsubsection{Discussion}

In this section, by using our Interaction Model, we described our first design concept of Timer Lamp and then subsequently discovered many design alternatives. These design possibilities could easily inspire three new design concepts of Timer Lamp, each of which can be integrated into the context better than the initial design concept. Further, we compared these new design concepts and generated a preferred design concept. This process proved the usefulness of our Interaction Model in three aspects, which are describing, discovering, and comparing SLS concepts. Also, we saw limitations that a general design guideline is still missing to instruct the use of our Interaction Model for designing SLS in a new design project through an entire design process. 


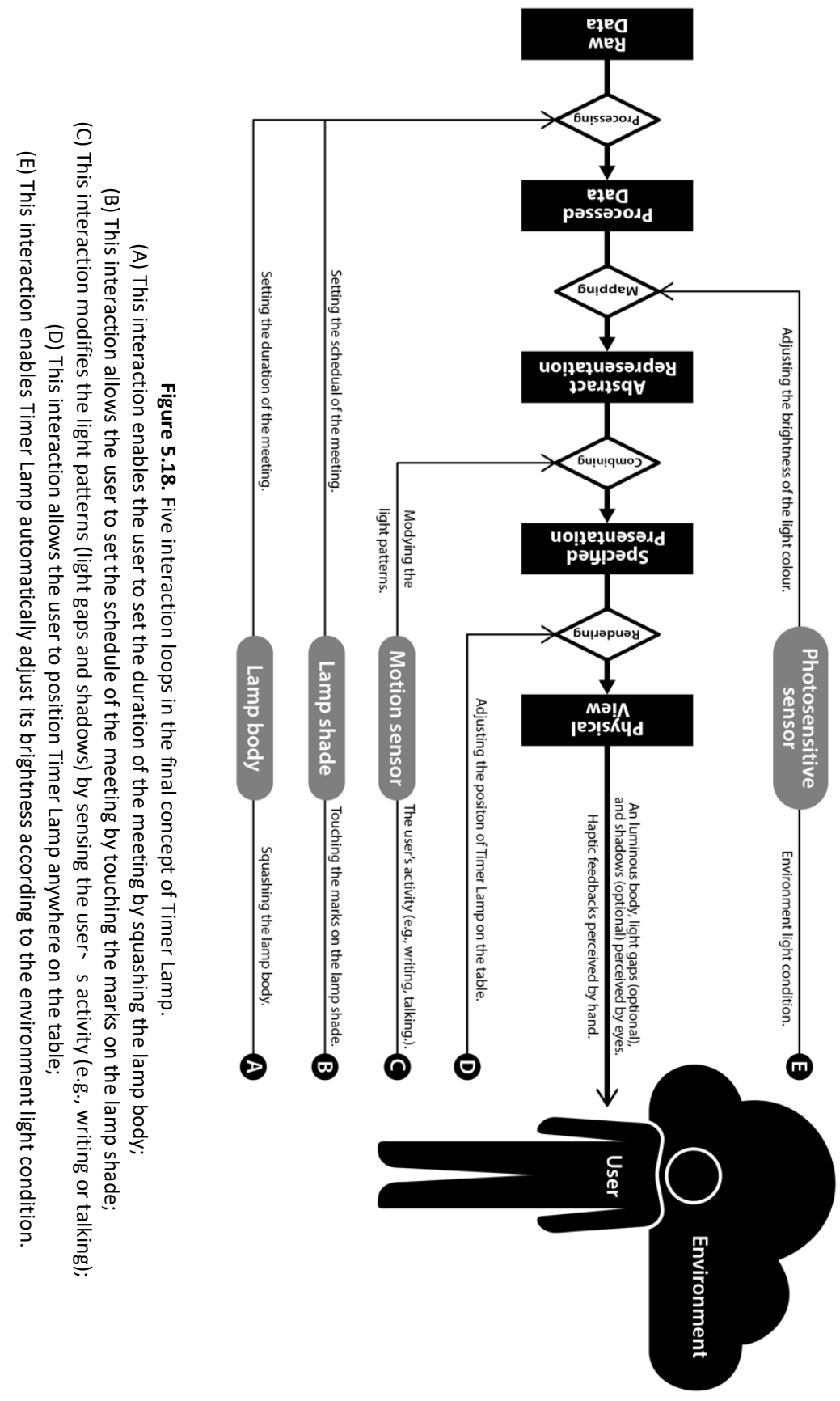




\section{Describing SLS concepts}

Our Interaction Model can help designers to describe their SLS concepts in a systematic way. Comparing with the general design descriptions, such systematic descriptions can provide designers a deeper understanding of their design concepts. Based on that, the other possible design concepts will emerge and probably be developed further.

\section{Discovering SLS concepts}

Our Interaction Model can challenge designers to explore new SLS concepts by questioning themselves: what are other design alternatives that were not used, why they were not used, and would they be better than the used ones? These questions will help designers to discover new design possibilities in a comprehensive way, especially in the conceptual phase of design, in which it does not make sense to just make decisions without considering other possibilities.

\section{Comparing SLS concepts}

Our Interaction Model can also help designers to compare their design concepts in a structural way. This is realized by comparing every design decision the designers have made in defining each of the data transformation steps or the interaction loops. It supports designers to gain sound arguments in either selecting better ideas or generating new ideas. Based on that, a reasonable better design concept can be identified to be further developed.

\section{Limitations of using the SLS Interaction Model}

In this validation process, we also noticed the limitation that we did not really give suggestions about how to use our Interaction Model to support designers in a new design project, i.e. how to integrate the use of the Interaction Model into a design process. Moreover, in this section, we only used our Interaction Model to generate concept ideas of SLS without further implementation and testing. Although the final concept seems reasonable better than the ones that we presented in section 5.4.3, it is not proved with user test in a real context. Therefore, we see a need to propose general design process/guidelines for designers to start a whole new design project of SLS.

\subsection{Conclusion}

In this chapter, we presented our SLS Interaction Model. This model aims to provide designers with a mechanism to argue about SLS design concepts. Also, we validated the usefulness of our Interaction Model by envisioning the further development of the initial design concepts of Timer Lamp (see section 2.2). The results proved that our Interaction Model is instrumental for designers to describe, discover and compare design concepts until a most promising design 
concept is selected. However, we also found out a missing part of using our Interaction Model for the design of SLS, which is general design process/guidelines. 



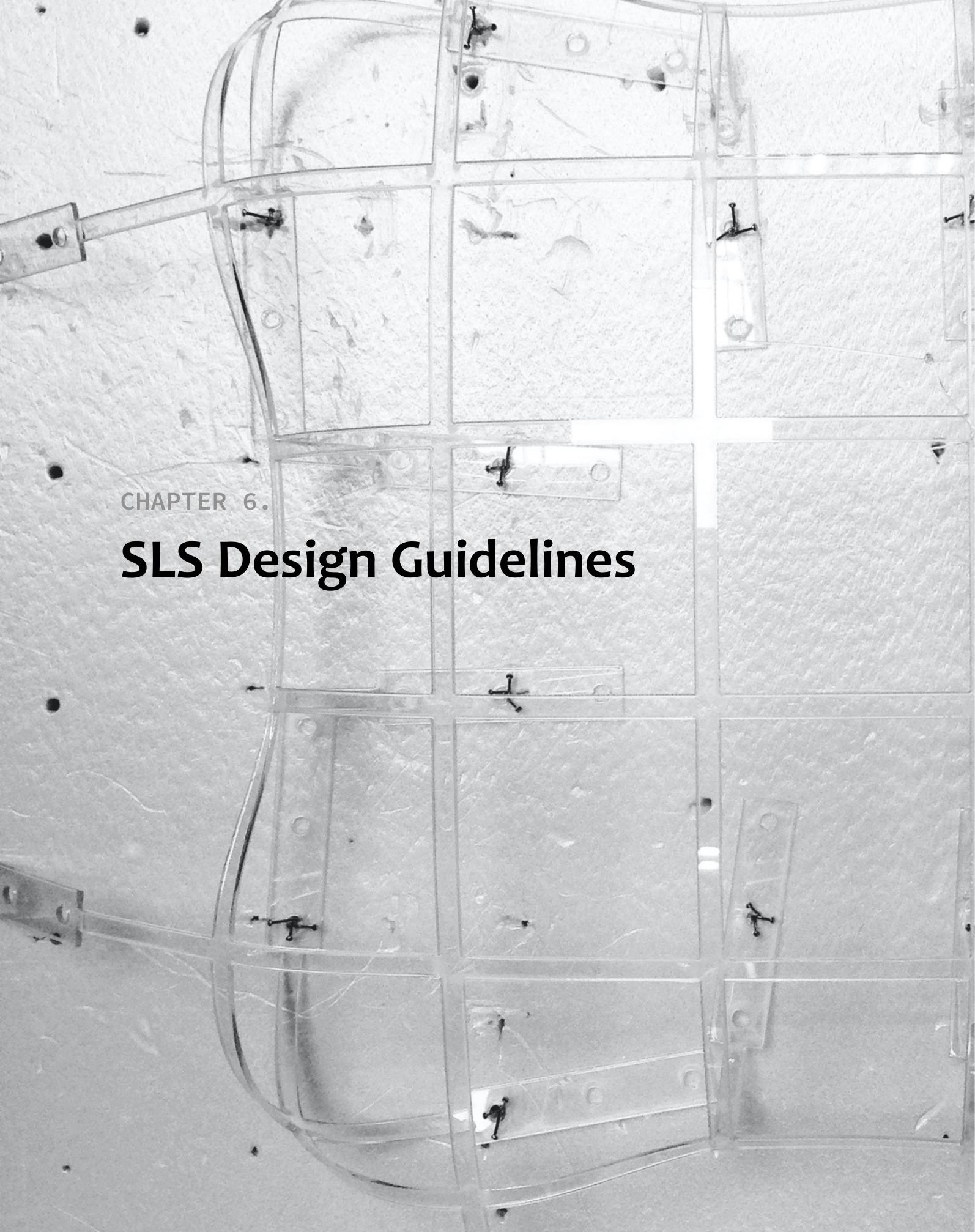

SLS Design Guidelines 
In the previous chapters, we have introduced the SLS Framework and Interaction Model. These promised to help designers to systematically develop SLS. The Framework provides an overview of design alternatives for SLS. The Interaction Model shows an overview of interaction alternatives when designing SLS for Information Decoration. By applying the SLS Framework and Interaction Model in our own design practice, we found them useful as ideation tools. However, a guidance of how to use these tools in the design process was still missing. Therefore, we propose the SLS Design Guidelines, which integrate the use of the SLS Framework and Interaction Model in a regular design process of ideation. This process is carried out through three ideation sessions: collecting clues, generating ideas, and completing concepts.

In this chapter, we will first introduce the goals of these three ideation sessions. Also, we will introduce the design brief for a hypothetical SLS that was used to demonstrate the use of our Design Guidelines. After that, we will elaborate on the Design Guidelines and how we have applied them in the three ideation sessions in the hypothetical example. Finally, we will discuss the possibilities and limitations of using our Design Guidelines by reflecting on the practical design exercise we did in the hypothetical SLS.

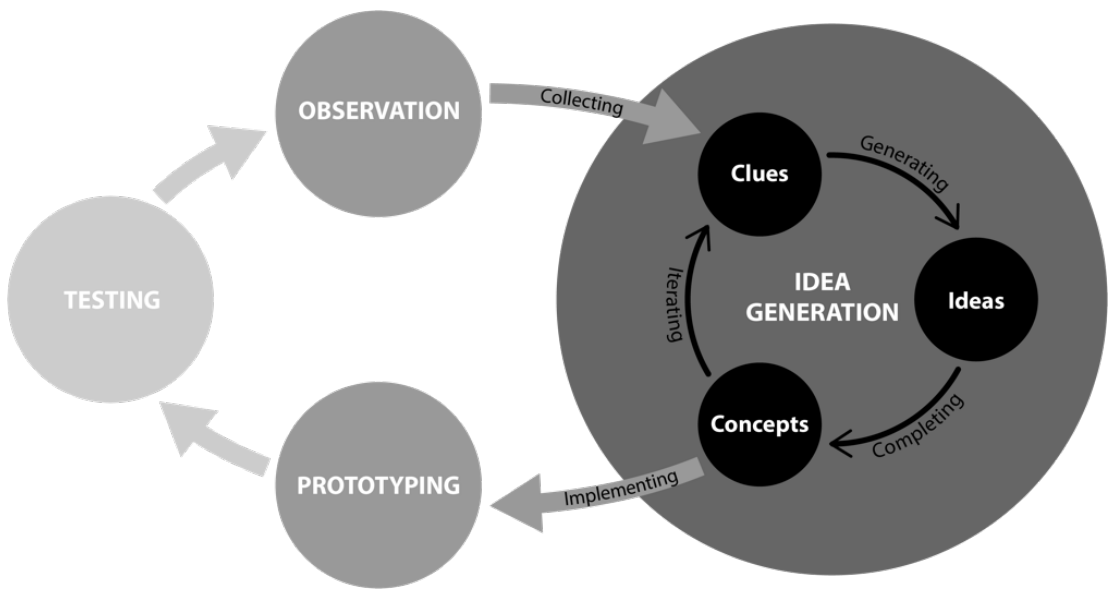

Figure 6.1. The grey parts: iterative cycle of human-centred design (Norman, 2002); the black parts: the three iterative sessions within the idea generation phase.

\subsection{Clues, Ideas, and Concepts}

The SLS Design Guidelines aim to support designers in the ideation phase of a humancentered design process. The ideation phase is positioned in between the design phases of understanding context (observation) and implementing concepts (prototyping), as 
represented in Figure 6.1. The ideation phase is split into three ideation sessions: collecting clues, generating ideas, and completing concepts. The human-centred design cycle has been proposed by Don Norman (Norman, 2002). The 'clues' session explores the opportunities for idea generation by analysing the context information collected from the observation phase; the 'ideas' session generates inspiring ideas that can possibly be further developed into concepts; the 'concepts' session completes the promising ideas into concrete SLS concepts. The three ideation sessions can be iterated multiple times. That means designers are free to repeat or re-evaluate previous sessions for collecting new clues or for generating more ideas. Designers will gain insights when they generate concepts, such insights support the next iteration.

\subsection{Designing a hypothetical SLS}

We will present and demonstrate the use of our SLS Design Guidelines with a hypothetical example. In this example, we develop SLS concepts to inform people working in a basement without windows about the rain forecast. The context information of this hypothetical project is described below.

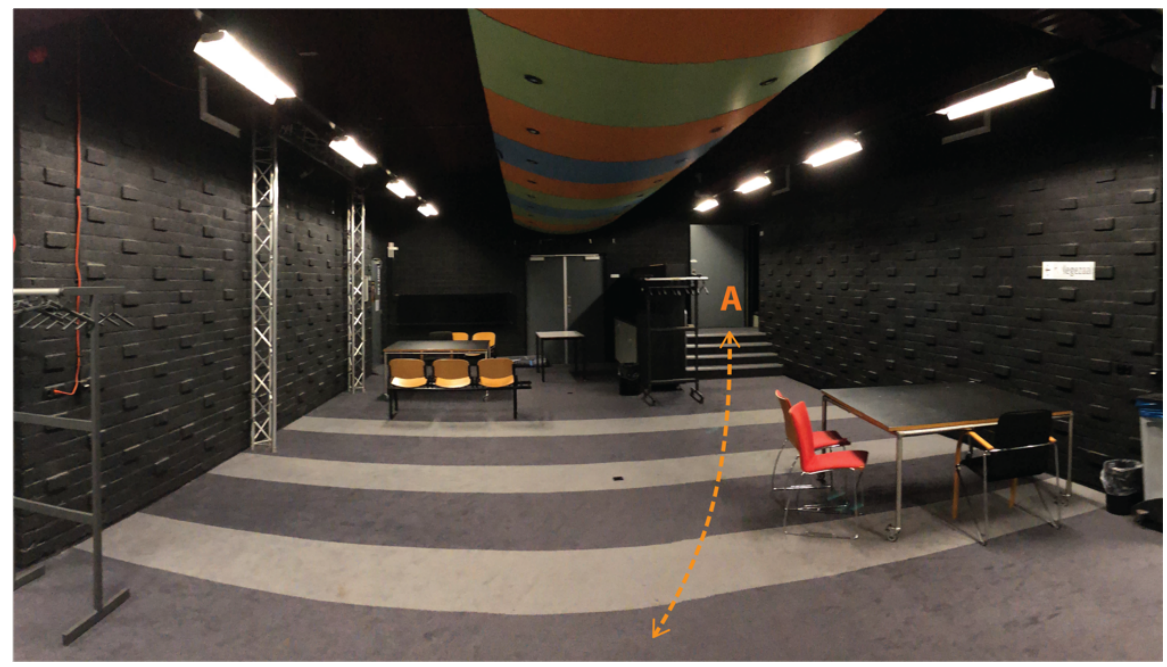

Figure 6.2. The open space in the basement. The orange arrow indicates the students' fixed route in the basement between the ground floor and the classroom.

The basement (see Figure 6.2) is an open space which is usually quiet unless there is a lecture in the classroom (Point A in Figure 6.2). Students might come from different departments of the university. Their activities in the basement can be individual or teamwork, prototyping, as 
well as entering or leaving the classroom (route, the orange curve in Figure 6.2). The students do care about the rain condition, especially when they are about to leave the building. However, it is impossible for them to check the rain condition by glancing through the outside window as usual. So, they can only check this online with an app, for instance Buienalarm ${ }^{17}$, which is very popular in the Netherlands.

\subsection{Design Guidelines}

In this section, we will introduce the SLS Design Guidelines for the three ideation sessions: collecting clues, generating ideas, and completing concepts. More specifically, we will elaborate on: what design activities are involved in the three sessions; how to apply the SLS Framework and Interaction Model in these design activities; how to reflect on the outcomes at each end of the three sessions. Also, we will demonstrate the use of the Design Guidelines by developing concepts in the hypothetical example mentioned in the previous section.

\subsubsection{Collecting clues}

In the first ideation session clues are collected that can provide designers with starting points in developing SLS concepts. These clues are collected from the results of the observation phase. Usually, the observed information could be ambiguous, which makes that this information cannot easy be used directly in the idea generation phase. By using our Interaction Model as a probe, the implicit observed information can be transformed into explicit design opportunities. For example, in our example, the information from "Buienalarm" indicates an alternative source for gathering Raw Data about rain forecast; the information about "an open space which is usually quiet" inspires us to add an Interaction Loop to turn off the information displays when nobody is using the basement; and the information about the "students' activities in the basement" implies that the Physical View of SLS should take the user's perspective along the fixed route (see the orange arrow in Figure 6.2) into consideration. We define all these types of opportunities as clues that can initiate and act as starting points for concept development.

\section{Raw Data}

Looking for possible information sources.

\footnotetext{
${ }^{17}$ Buienalarm. Retrieved on 29.06.2019 from https://www. buienalarm.nl
} 


\begin{tabular}{|l|}
\hline Processing and Processed Data \\
\hline Investigating the specific information that might interest the user most. \\
\hline Mapping and Abstract Representation \\
\hline $\begin{array}{l}\text { Looking for opportunities for representing the information by means of light change and } \\
\text { shape change. }\end{array}$ \\
\hline Combining and Specified Presentation \\
\hline $\begin{array}{l}\text { Looking for opportunities for presenting the information with light patterns in an aesthetic } \\
\text { and meaningful way. }\end{array}$ \\
\hline Rendering and Physical View \\
\hline $\begin{array}{l}\text { Looking for opportunities for integrating the Information Decoration into the physical } \\
\text { environment. }\end{array}$ \\
\hline The interaction loops from the user side \\
\hline Looking for opportunities for adapting the Information Decoration to the user's activities. \\
\hline The interaction loops from the environment side \\
\hline $\begin{array}{l}\text { Looking for opportunities for adapting the Information Decoration to changes in the } \\
\text { environment. }\end{array}$ \\
\hline
\end{tabular}

Table 6.1: Design Guidelines for collecting clues to develop SLS concepts.

To collect clues such as the ones mentioned above, we recommend designers to analyse the context information through seven design activities presented in Table 6.1. These design activities are mainly related to the elements of information flow (see section 5.2.2) in the SLS Interaction Model. Actually, the information flow exists in every SLS system only in different forms. The elements that are related to the interaction loops (see section 5.3.1) are optional, which depend on the requirements of adapting the SLS to the changes in the environment or the needs of the users. Although the design activities for collecting clues are presented in a specific order, according to the information flow in the model, they are not intended to be carried out in sequence. Designers are free to start from any design activity based on their own interests or expertise. Also, they can iterate these seven design activities to collect sufficient clues. 


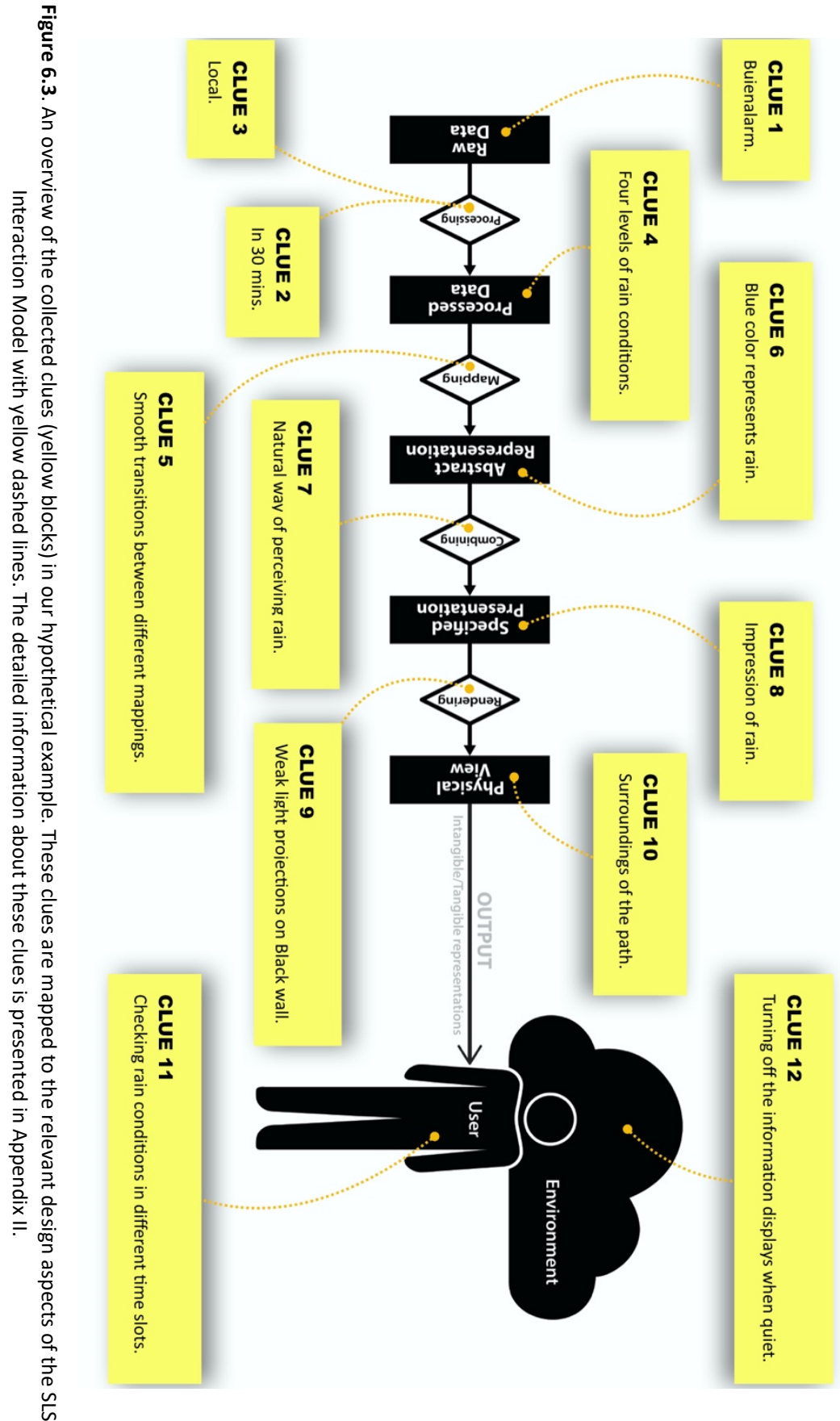


To have a better overview of the collected clues, designers can visually integrate the clues to the layout of our Interaction Model. For instance, Figure 6.3 presents the clues we collected in our hypothetical example by following the guidelines introduced in Table 6.1. The detailed explanation of these clues can be seen in Appendix II. By presenting this kind of overview, designers can intuitively reflect on both the quantity and quality of the collected clues. For the quantity part, designers can check whether the collected clues have involved all the essential design aspects of SLS. This can stimulate designers to collect more clues so that they can understand the context sufficiently good. For the quality part, designers are encouraged to reason using the collected clues so that they can formulate stronger argumentation when proposing their final concepts.

In our hypothetical example, we intentionally present clues roughly to leave more space when we refer to them in further ideation sessions. For instance, the initial version of CLUE 11 (see Appendix II) was enabling the user to check the rain forecast for different time slots, which was already concrete and might limit the idea generation later. Thus, we made it more abstract, presenting a potential interaction that enables the user to check the rain forecast based on his/her own needs. Eventually, we developed CLUE 11 into CONCEPT 1.10 (see Appendix II), which allows the user to check the rain forecast in three modes: the current rain condition for Eindhoven; the rain condition after 30 mins at 's Hertogenbosch; the rain condition after 90 mins at Utrecht. Obviously, the final concept not only includes the time slots in the initial version but also has extra information for location.

\subsubsection{Generating ideas}

The second ideation session is to generate ideas based on the collected clues. These ideas relate to the same element of the Interaction Model as the clues they originate from. The element could be Abstract Representation, Specified Presentation, or Physical View. For instance, in our hypothetical example, we came up with an idea of "producing rain-drop like reflected light patterns to display the rain condition", which relates to the Specified Presentation of SLS. Also, the ideas generated in this ideation session can involve potential Input and Update signals in some possible interaction loops. For instance, we thought of an interaction that automatically alters the representation fidelity of SLS by sensing the sound level in the basement. This idea specifies an interaction loop from the environment side towards Mapping. While, the other design aspects (Raw Data, Processing, Processed Data, Mapping, Combining, Rendering, and Interfaces) are more about detailing the ideas. We do not recommend designers to consider these detailed aspects too much, which might restrict creativity in this diverging stage. 
In Table 6.2, we present the Design Guidelines for generating ideas of SLS through four clusters of design activities. The four clusters are corresponding to the four (re)presentation aspects (Abstract Representation, Specified Presentation, Physical View) and an optional aspect (interaction loops) in SLS. The design activities indicate what alternative elements of SLS can be considered when generating ideas for each of the four mentioned aspect. These elements include four styles of SLS (section 3.4), five basic components of SLS (section 3.3.1 and section 4.1), three types of attributes of SLS (section 3.3.2), and three kinds of light patterns of SLS (section 3.3.3). Also, these elements involve the alternative techniques (section 5.3.2) that can support adding possible interaction loops (5.3.1). These alternative elements can support designers in both seeking inspirations and describing ideas in a systematic way.

\section{Abstract Representation}

- Selecting the possible Styles of SLS that can fit in the given context.

- Selecting the suitable types of Light Attribute or Shape Attribute for the SLS components for information representation.

- Explaining the meaning of the information representation.

\section{Specified Presentation}

- Selecting the possible Light Patterns that are suitable for information presentation in the given context.

- Depicting the expression and meaning of the specified light pattern.

- Drawing a scheme of what specific shape changes and light changes are needed to produce the light pattern.

- Considering the use of Extra Attributes to optimize the information presentation that involves Preset Objects.

\section{Physical View}

- Thinking about utilizing the existing light sources in the environment as Active Lights or Passive Lights.

- Thinking about utilizing the existing physical objects in the environment as Preset Objects.

- Trying out the light patterns with simple experiments in reality or by computer simulation.

Interaction loops

- Selecting the interaction loops that are worth to add. 
- Thinking about what possible Controls are needed to trigger the added interaction loops.

- Selecting the suitable interaction techniques to support the added interaction loops.

- Thinking about integrating Preset Objects with the Interfaces in the added interaction loops.

Table 6.2: Design Guidelines for generating ideas to compose SLS concepts.

In this session, designers can carry out these four clusters of design activities in any order based on their own interests or expertise. However, we recommend designers to review all these design activities thoroughly through multiple iterations. Meanwhile, designers can have an overview of their generated ideas by visually mapping their ideas to the relevant aspects in the layout of our Interaction Model. For example, in Figure 6.4, we present an overview of 17 ideas that we generated in this session. The specific information can be found in Appendix II. We believe that such kind of overview could stimulate designers to generate various ideas from a broader perspective rather than focus on detailing one or two ideas. Also, this overview can easily inform designers which aspects of the concepts need to be completed in the next ideation session.

In our hypothetical example, we gained inspirations by searching for the impression of rain on Google, reviewing the light effects in the relevant examples of SLS (see Appendix I), and reflecting on the use of our previous experimental prototypes. For instances, IDEA 8 was inspired by the images about umbrella we saw on Google, IDEA 11 referred to an existing example of SLS (see Appendix I, No. 20), and IDEA 17 learned from the interaction we explored before by Press Light ${ }^{18}$. Once we came up with an idea, we described it with the alternative elements defined in SLS Framework, which stimulated us to think about more alternative ideas by reflecting on the possible use of the other alternative elements. For example, IDEA 1 is about Objects-driven SLS. Based on that, we thought of the possibilities of applying Active SLS (see IDEA 2) and Lights-driven SLS (see IDEA 3). For another example, IDEA 12 is about utilizing the ceiling lamps as Passive Lights. Based on that, we were aware of the spotlights (see IDEA 13) on the ceiling as well, which could be modified as Active Lights (e.g., beamer) for representing information in a rich way.

\footnotetext{
${ }^{18}$ Jiang Wu (2017). Press Light. Retrieved on 29.06.2019 from https://vimeo.com/274491229
} 


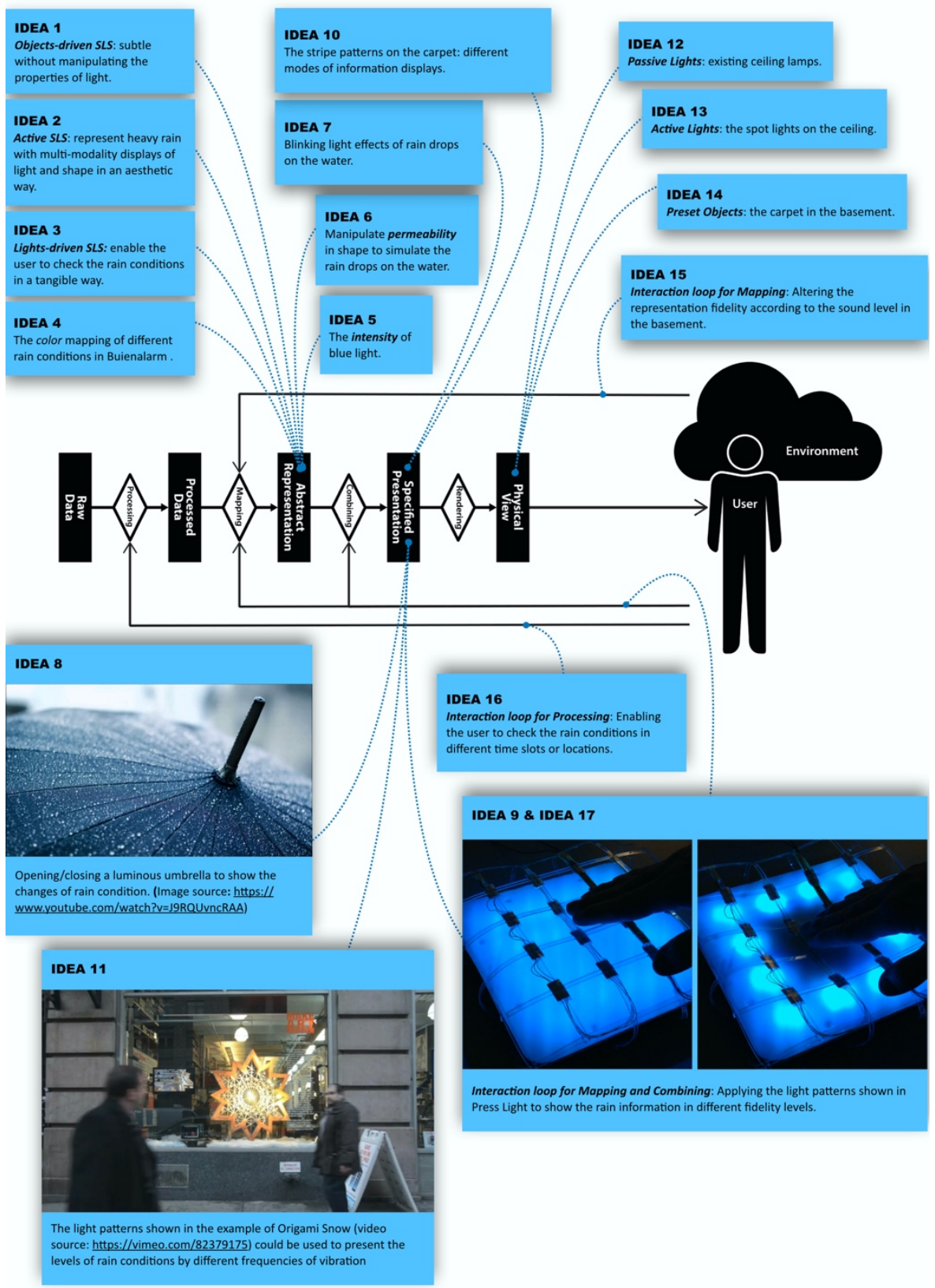

Figure 6.4. An overview of the selected ideas (blue blocks) in our hypothetical example. These ideas are mapped to the relevant aspects in the SLS Interaction Model with blue dash lines. The detailed information about these ideas is presented in Appendix II. 
At the end of this session, designers could generate a large number of ideas. It is not necessary to develop all of them further into concrete concepts. Instead, we recommend designers to select some promising ideas that better fit in the context or match the design requirements. In general, according to the definition of SLS (see section 3.2.5), we propose four basic criteria that can support this selection session, including subtleness, unobtrusiveness, aesthetics, and environmental appropriateness.

In fact, we got many ideas by following the guidelines for generating ideas in our hypothetical example. The ideas presented in Figure 6.3 were just 17 selected ones that we considered them to better fit the criteria mentioned above. For instance, we excluded an idea of interaction that allows the user to check the rain condition in different locations by speaking out the city's name in front of the information display. We figured this idea could be intrusive if there's a lecture next door (point A in Figure 6.2) in the basement.

\subsubsection{Completing concepts}

The last ideation session aims to detail the ideas generated in the previous session into concrete concepts. More specifically, designers need to define the complete information flow and the interaction loops of SLS. To define the information flow aspects, designers will need to figure out how to realize the generated ideas in their data transformation steps, including Processing, Mapping, Combining, and Rendering. For instance, in the second session, we proposed an idea for the Specific Presentation (see IDEA 8 in Appendix II) aspect. In this session, we defined its Combining aspect by CONCEPT 1.4 (see Appendix II). To define the interaction loops aspects, designers will specify the needed Input (user controls and environment controls) and Interface (or sensors). For instance, IDEA 16 (see Appendix II) was finally detailed as CONCEPT 1.10 (see Appendix II).

In this session, we recommend designers to carry out four clusters of design activities shown in Table 6.3. These design activities specify what decisions need to be made for detailing the generated ideas into concrete concepts. Designers can start from any of these design activities based on their own interests and expertise, however, they need to finish all of them until the concrete concepts are ready to be implemented. To do so, designers can combine the generated ideas into concepts. Also, they are free to go back to the previous two ideation sessions when they find out new ideas or clues in this session.

\section{Mapping}


Defining the specific Mappings between the information changes and the variable attributes of manipulating the Active/Passive SLS components.

Combining

- Defining the deployments of all the employed SLS components to realize the desired light patterns.

- Defining the material properties of all the employed SLS components to realize the desired light patterns.

- Defining the specific variables of every Abstract Representation.

- Defining the specific attributes of Preset Objects.

\section{Rendering}

- Defining the position where the Specified Presentation will be implemented in the environment.

- Defining the shape-changing actuations that can be used for Active Objects.

- Defining the light sources that can be applied to Active Lights.

- Defining the fabrication methods of the Specified Presentation.

Interaction loops

- Defining the specific Controls for the added interaction loops.

- Defining the specific Interfaces for the added interaction loops.

- Defining the specific sensors that are needed to realize the added interaction loops.

- Defining the specific Update of the altered data transformation steps when the added interaction loops happen.

Table 6.3: Design Guidelines for completing SLS concepts.

Completing concepts needs available and feasible techniques that can bring the ideas into reality. In the previous chapter, we have introduced multiple alternative techniques to implement the information flow (section 5.2.3) and the interaction loops (section 5.3.2) of SLS. Although these techniques were described in general terms, they could be a useful reference for designers to complete concepts. For implementing the information flow, the alternative techniques include six means of creating light patterns (emitting, blocking, diffusing, reflecting, refracting, and projecting) for Combining, and six available techniques for shape-changing actuation (electromagnetic, electromechanical, hydraulic, piezoelectric, pneumatic, smart materials) for Rendering. For implementing interaction loops, the alternative techniques include two means of user controls (explicit controls and implicit controls), two kinds of 
environment controls (climate changes and context changes), three types of interfaces (Graphic User Interfaces, Tangible User Interfaces, and Nature User Interfaces) and multiple feasible sensors (sound sensors, photosensitive sensors, force sensor, motion sensors, etc.).

To have a clear overview of the concept concretization process, designers can also use the structure of our Interaction Model to visually present every definition they made in this ideation session. This overview can help designers to intuitively check which aspects of their concepts are not detailed (enough) yet. Also, this overview could support designers easily integrating their generated ideas into concepts.

In our hypothetical example, we present one completed concept in Figure 6.5. The detailed information about this concept can be seen in Appendix II. This concept was developed based on the combination of IDEA $2,4,5,8,10,14,15$, and 16 . In this ideation session, we detailed these ingredient ideas with specific variables and parameters. For instance, CONCEPT 1.3 detailed the colour Mapping proposed in IDEA 4. Also, we figured out which techniques can be applied to realize these ingredient ideas in an appropriate way. For instance, CONCEPT $\mathbf{1 . 1 0}$ indicates the specific sensors and user controls in the interaction proposed in IDEA 16. Moreover, CONCEPT 1.4 and CONCEPT 1.8 specify a new way of implementing the luminous umbrella by weaving the fabric and optical fibres together. We considered it to produce richer and more aesthetic light patterns than just embedding the light sources inside the umbrella's frame to produce diffused light patterns.

By the end of this session, designers could have some concrete concepts. However, these concepts are still in fantasy, which could be failed in implementation by different realistic reasons. In this sense, before diving into the prototyping phase, we recommend designers to generally evaluate their concepts by some specific criteria, which are set by themselves according to the design context. By doing so, designers can systematically select the best concepts that can be further implemented. More importantly, designers can be aware of either the values or the shortcomings in their concepts. The values support sound arguments for designers to propose their concepts convincingly. The shortcomings might lead to another iteration in the ideation phase.

In our hypothetical example, the concept presented in Figure 6.5 was not accomplished at once by just following the guidelines one by one. In fact, it was polished in several iterations by reflecting on its functionality, feasibility, and extra values. For instance, we thought about two ideas for Combining: one is to utilize the spotlights (based on IDEA 13) on the ceiling to project coloured images on the umbrella fabric; the other is to integrate the RGB LED strips with the frame of umbrella. The first idea was not adopted because the projected light patterns could be unstable when the user moves the umbrella to different places. Also, changing the 


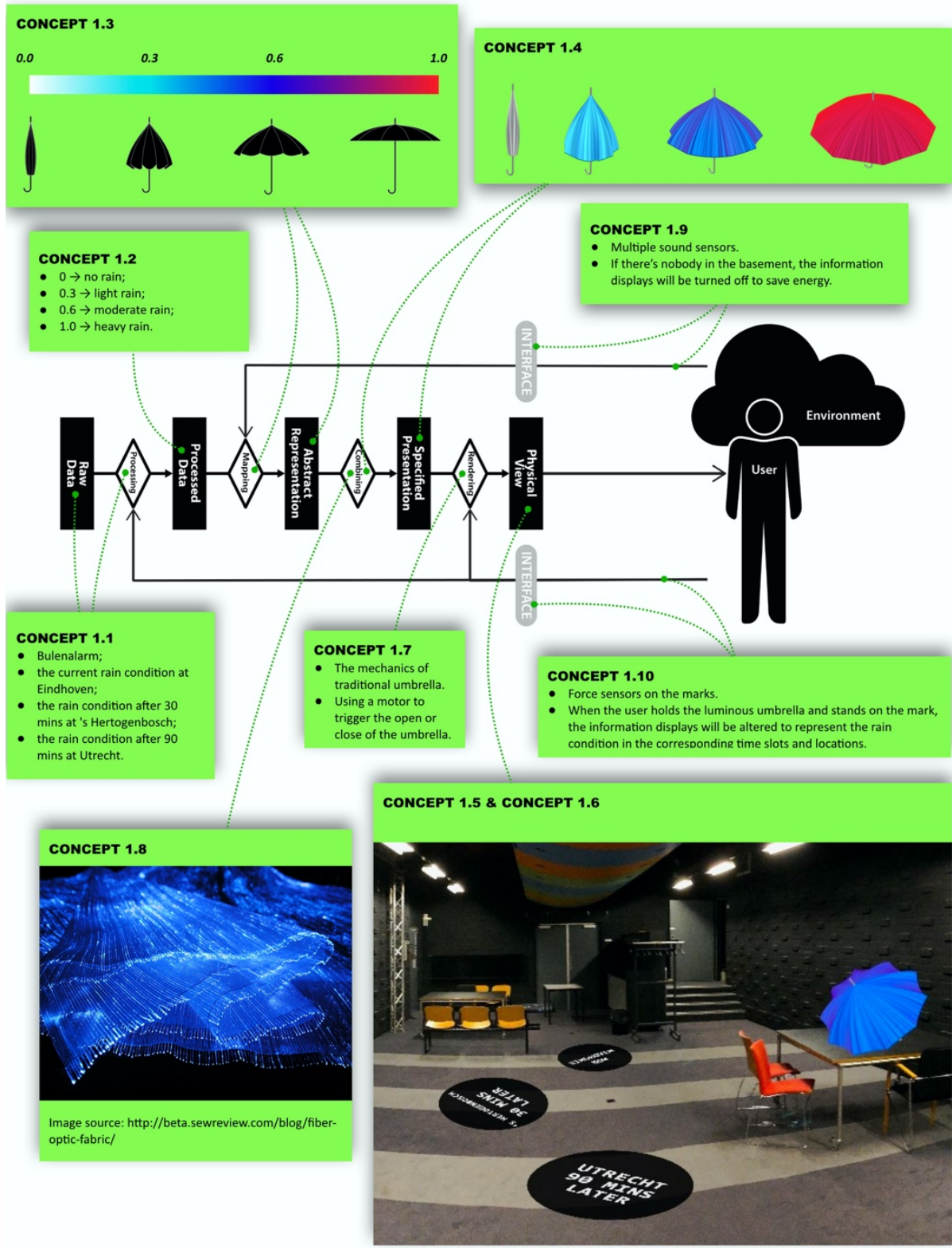

Figure 6.5. An overview of the details (green blocks) of a final concept in our hypothetical example. These details are mapped to the relevant aspects in the SLS Interaction Model with green dash lines. The detailed information about this concept is presented in Appendix II. 
spotlights with powerful RGB light sources may not be feasible. For the second idea, it is feasible indeed but lacks extra values. When we tried other available techniques for Combining, we found a better one (see CONCEPT 1.4 in Appendix II). The randomly distributed micro brighter points on the umbrella fabric could remind the users of the rain drops, which can be more elegant and appropriate to portray the rain information.

\subsection{Discussion}

In the previous sections, we have introduced three ideation sessions (collecting clues, generating ideas, and completing concepts) to develop SLS concepts. Moreover, we proposed Design Guidelines for designers to carry out these three ideation sessions. These guidelines integrate the design aspects and design alternatives, which are provided in the SLS Framework and the Interaction Model, into different design activities for concept development of SLS. Also, we have demonstrated the use of these guidelines by developing concepts in a hypothetical example. In this section, we will reflect on the use of our Design Guidelines in the hypothetical example. Based on that, we will discuss the potentials and limitations of using our Design Guidelines in design practice.

\subsubsection{Potentials of using the SLS Design Guidelines}

According to our practice in the hypothetical example, we found the largest benefit of the Design Guidelines was that they provide a systematic and informative overview to support concept development of SLS. This overview indeed guided us to think about what design alternatives (elements or techniques) could be considered in which aspects of SLS and for what design activities. Moreover, this overview supported us with a rational ideation process from three ways: analysing concepts, reflecting on concepts, and contextualizing concepts.

Firstly, analysing the concepts in a systematic way helped us to compare our concepts. From this, we can make decisions rationally rather than intuitively. For example, CONCEPT 1.3 (see Appendix II) was developed by first comparing IDEA 4 and IDEA 5 (see Appendix II), and then combining their merits into one. IDEA 4, representing rain condition with the intensity changes of blue light (generated from CLUE 6) can be subtle, although that might only fit when the rain is not heavy, and IDEA 5, representing the heavy rain information with red colour (inspired by CLUE 1) makes sense because the users may not want to miss that information.

Secondly, this analytical thinking can prompt us to reflect on the elements that our concepts consist of, thereby considering alternatives. For example, based on IDEA 1 (see Appendix II) 
which is about Objects-driven SLS, we thought of the possibilities of applying Active SLS (see IDEA 2) and Lights-driven SLS (see IDEA 3). For another example, when completing a concept for Combining, the first idea we got was using umbrella fabric to diffuse the light embedded on the umbrella frames. Although that idea is feasible, we tried to think about the other alternative ways of realizing the luminous umbrella effects. As result, we got CONCEPT 1.4 (see Appendix II) which creates a kind of mixed light patterns by applying both the diffusing and the emitting techniques (see Figure 6.5).

Thirdly, the systematic overview continuously reminded us to think of the context rather than to dive into a specific aspect. As a result, we came up with several ideas and concepts of SLS that are potential can be seamlessly integrated into the environment. For instance, the stripe patterns on the carpet inspired us to an idea related to Physical View (see IDEA 10). Further, this IDEA 10 became a key inspiration for CONCEPT 1.1, CONCEPT 1.5, and CONCEPT 1.10 (see Appendix II).

\subsubsection{Limitation of using the SLS Design Guidelines}

By performing the hypothetical design exercises in the example, we also noticed that our Design Guidelines are informative but still in an abstract sense. Although our Design Guidelines indicate diverse design activities (see Table 6.1, Table 6.3, and Table 6.5) in ideation processes, they are more about 'what to do' but less about 'how to do'. For instance, the guideline of "selecting the interaction loops that are worth to add" only reminded us to consider about what are the possible interactions in the context, but not about how to select the possible interactions loops under different circumstances. Thus, we just explored all the alternative interaction loops one by one in the hypothetical example. Of course for making decisions and developing interesting and useful designs for SLS requires practical experience. In the future, it might be possible to integrate such practical insights, which might lead to more specific guidelines.

\subsection{Conclusion}

In this chapter, we introduced a systematic approach to develop SLS concepts through three ideation sessions, which are called collecting clues, generating ideas, and completing concepts. For each of the three sessions, we proposed Design Guidelines for designers to carry out idea generation for different aspects of SLS using the design alternatives provided in the SLS Framework and the opportunities of the Interaction Model. By reflecting on the use of our Design Guidelines in a hypothetical example, we found that the Design Guidelines can indeed 
provide a systematic and informative overview to support the ideation process in an analytical, reflective, and contextualized way. However, these informative Design Guidelines are still abstract and might lack a certain level of practical guidance. This is caused by our limited experience of developing SLS in practice.

To validate the usefulness of the Design Guidelines and to gain more insights on the future development of the Design Guidelines, we have conducted an evaluation study, which will be introduced in the next chapter. 

CHAPTER 7 .

Evaluating the SLS Framework and the SLS Interaction Model 
In the previous chapters, we have introduced the SLS Framework and the SLS Interaction Model. These two formal descriptions of understanding SLS, aim to support designers in the systematic development of SLS concepts. Also, in chapter 6, we proposed Design Guidelines, providing an approach to use the Framework and the Interaction Model in the ideation process. In our design explorations, we found that the Design Guidelines, the Framework and the Interaction Model are useful in generating alternative ideas and in reflecting on existing and new design concepts. In this chapter we perform a user study to evaluate the SLS Framework and Interaction Model in a design setting. In this study, we invited ten experts to review and use the Framework and the Interaction Model by following the Design Guidelines in a hypothetical design project.

We first define the evaluation goals and how we want to achieve them. Then, we introduce the methodologies applied for evaluation and data analysis. Next, we show the results of the evaluation from different sources. After that, we present the findings of the evaluation by interpreting the results. Finally, we discuss the limitations of the study and use the findings to reflect on the goals of the study.

\subsection{Evaluation Goals and Approach}

Primarily, this study was intended to validate the usefulness of the SLS Framework and the Interaction Model. Because SLS and the tools to design them were new to the participants, it was necessary to ensure a proper understanding of the Framework and the Interaction Model first. Next to exploring the utility and the potential benefits, we also wanted to derive recommendations for future improvement of the Framework and the Interaction Model. To achieve these goals, we took the following evaluation approaches.

\section{Investigating the understandability of the SLS Framework and the Interaction Model}

Evaluating the usefulness of the Framework and the Interaction Model when these are understood insufficiently, does not make sense. Therefore, in this evaluation study, we first investigated whether participants actually do understand the Framework and the Interaction Model after providing instructions We discuss the results to correct participants' misunderstandings of the Framework and the Interaction Model.

\section{Validating the usefulness of the SLS Framework and the Interaction Model}

The primary goal of the study is to validate the usefulness of the Framework and the Interaction Model. More specifically, we assumed the usefulness is in generating alternative ideas and reflecting on SLS concepts in the ideation phase of designing SLS. Ideally, the best 
evaluation approach would be offering the Framework and the Interaction Model to multiple designers in a real SLS design project, but that was not feasible. Therefore, we invited experts to review and use the Framework and the Interaction Model by following the Design Guidelines in a hypothetical SLS project. By means of the Design Guidelines (see chapter 6), we provided an overview instruction of how to use the Framework and Interaction Model in different ideation sessions (collecting clues, generating ideas, and completing concepts). We assume that when experts consider following the Design Guidelines is helpful, the usefulness of the Framework and the Interaction Model is confirmed.

Looking for possibilities to improve the usefulness of the SLS Framework and the Interaction Model

We also aimed to propose recommendations for improving the usefulness of the Framework and the Interaction Model. On the one hand, we investigated the causes for which the Framework and the Interaction Model are considered less useful. On the other hand, we evaluated the use of Design Guidelines to find out the possibilities of supporting designers in better using the Framework and the Interaction Model in the future.

\section{Exploring the potential benefits of the SLS Framework and the Interaction Model}

The Framework and Interaction Model were proposed to support designers in systematically developing SLS concepts. Besides the usefulness of generating alternative ideas and reflecting on the concepts, we attempted to explore other applications of the Framework and the Interaction Model. To do so, we also discussed some open questions with the participants to reveal the potential benefits of the Framework and the Interaction Model.

\subsection{Evaluation Method}

To achieve the goals mentioned above, we conducted a formal user study in which we invited ten experts to review and use the Framework and the Interaction Model by following the Design Guidelines in a hypothetical design project. We collected data by four means, questionnaires, comments, practical exercises, and interviews. In this section, we introduce how we collected and analysed the data in this study.

\subsubsection{Participants}

Ten experts participated in our user study. They are part of an academic design environment and all have both design and research expertise in areas related to SLS, such as interactive lighting, shape-changing interfaces, and ambient displays. All participants have experiences 
in educating design students. By asking the participants to reflect on their educational experiences, we expected some feedback on how useful the Framework and the Interaction Model are if we offered them to the design students in the future. The participants were between 29 to 44 years of age. Half of them have eastern culture backgrounds, and the other half are western culture based.

\subsubsection{Procedure}

From the participant's point of view, there are two phases in the evaluation. The first phase (grey blocks in Figure 7.1) is about learning the Framework and Interaction Model, the second phase (black blocks in Figure 7.1) is about using the Framework and the Interaction Model by following the Design Guidelines in a hypothetical design project. In each of these two phases, participants were asked to individually finish three activities guided by an instruction booklet. These activities include reading the instructions, doing the practical exercises, filling in the questionnaire and leaving comments. At the end of each phase, we invited the participants to join a personal interview to explain their comments and to answer some extra questions.

\section{Instructions}

Participants were asked to read instructions before they went into the practice sessions. The first instruction explains the definition of SLS, the SLS Framework, and Exercise 1. The second instruction introduced four SLS design cases, the SLS Interaction Model, and Exercise 2. The last instruction introduced the design brief of a hypothetical project, the Design Guidelines, and Exercise 3. We presented these instructions in a 55-page instruction/work booklet. To avoid taking too many efforts from the participants, we tried to keep the explanations of the Framework and Interaction Model as short as possible. Also, we provided many examples and visual representations to help the participants to understand the contents of the Framework and the Interaction Model. The details of the booklet are presented in Appendix III.

\section{Exercises}

There are three exercise sessions in this study. By means of Exercise 1 and Exercise 2, we intended to check the understanding of the participants on the Framework and the Interaction Model. More specifically, in Exercise 1 (from page 16/55 to page 18/55 in Appendix III), participants were asked to describe an example (Lungplant (see Appendix I, No. 15)) of SLS by using the vocabulary offered by the Framework. In Exercise 2 (from page 33/55 to page 38/55 in Appendix III), participants were asked to firstly describe another actual example 


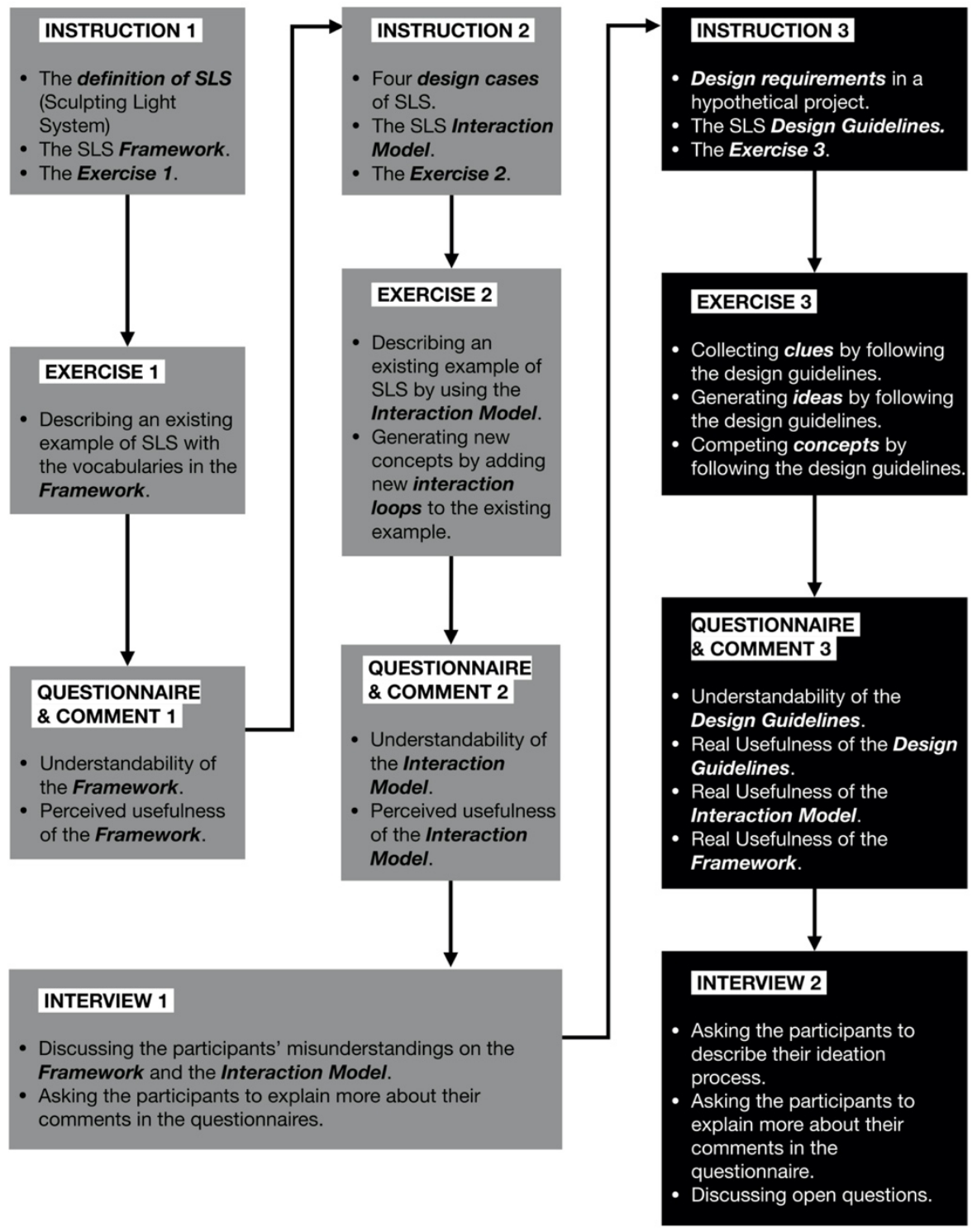

Figure 7.1. An overview of the evaluation procedure. The grey blocks are the activities carried out in the first phase. The black blocks are the activities carried out in the second phase. 
(Time Glow ${ }^{19}$ ) of SLS with the vocabulary used in the Interaction Model and then add new interaction loops to develop new concepts. In the interviews after these two practical exercises, we discussed misunderstandings by the participants if we saw they used the wrong vocabularies or added inaccurate interaction loops.

By means of Exercise 3 (page 44/55, page 47/55, and 50/55 in Appendix III), we evaluated the uses of the SLS Framework and the Interaction Model in an entire ideation process. In this practice session, participants were asked to develop SLS concepts in a hypothetical project (the same one as presented in section 6.2) by following the Design Guidelines in three ideation steps, which are collecting clues, generating ideas, and completing concepts. Meanwhile, we asked the participants to present their collected clues, generated ideas, and completed concepts on three different pages in the booklet.

\section{Questionnaires and Comments}

After each exercise the participants were asked to rate statements about the understandability and the usefulness of the Framework, the Interaction Model, or the Design Guidelines respectively on a 5-point scale of agreement questionnaire. Additionally, the participants were asked to leave comments for explaining their ratings of each statement.

By means of the questionnaires and comments of the first two sessions (see page 19/55, page 20/55, page 39/55, and page 40/55 in Appendix III), we mainly investigated the understandability and the perceived usefulness of the Framework and the Interaction Model. Firstly, we checked whether the participants understood our explanations of the Framework (Q1.1) and the Interaction Model (Q2.1). Then, we asked the participants, from an educator's point of view, to predict whether the Framework (Q1.2) and the Interaction Model (Q2.2) would be understandable for the other designers. After that, we asked the participants, from an expert's point of view, to review the assumed usefulness of the Framework and the Interaction Model with respects to systematically developing concepts (Q1.3 and Q2.3), generating many alternative ideas (Q1.4 and Q2.4), and reflecting on design concepts (Q1.5 and Q2.5). With these reviews, we were able to check the perceived usefulness of the Framework and the Interaction Model without addressing the Design Guidelines explicitly.

By means of the questionnaires and comments of the third session (from page 52/55 to page 55/55 in Appendix III), we first checked the understandability of the Design Guidelines (Q3.1 and Q3.2). Afterwards we focused on evaluating the real usefulness of the Framework and the

\footnotetext{
${ }^{19}$ Jiang Wu (2016). Time Glow. Retrieved on 29.06.2019 from https://vimeo.com/275524164
} 
Interaction Model. More specifically, we asked the participants to rate the statements (from Q3.3 to Q3.11) about the usefulness of the Framework or the Interaction Model in various ideation steps regarding collecting clues (see section 6.3.1), generating ideas (see section 6.3.2), and completing concepts (see section 6.3.3). Although some of the subjects in these statements are Design Guidelines, we intended to investigate the usefulness of the Framework and the Interaction Model. Design Guidelines were only meant to inform participants what design activities are involved when using the Framework and the Interaction Model to develop concepts in an ideation phase.

\section{Interviews}

At the end of each phase of the study, we invited participants to join an interview session individually. During Interview 1, we first discussed the participants' misunderstandings on the Framework and the Interaction Model according to their outputs of Exercises 1 and 2. By doing so, we tried to ensure that every participant had a reasonable knowledge of the Framework and the Interaction Model before he/she proceeded to the second phase of the evaluation. Then, we asked the participants to explain more about their comments on Questionnaire 1 and Questionnaire 2. In the second interview, we first asked the participants to describe how they developed concepts in the hypothetical design project by following the Design Guidelines. After that, we asked the participants to explain more about their comments on Questionnaires 3. Finally, we discussed some open questions (see Table 7. 1) with the participants about the potential benefits of applying the knowledge of SLS.

Who will benefit from the knowledge of SLS?

What kinds of projects will benefit from the knowledge of SLS?

Would you like to apply the knowledge of SLS to your work in the future?

Do you have any other comments on the knowledge of SLS?

Table 7.1. The open questions for Interview 3.

\subsubsection{Analysis}

In this study, we collected both quantitative and qualitative data. The quantitative data include the ratings from the three questionnaires and the outputs from the three exercises. The qualitative data refer to the remarks related to the Framework, the Interaction Model, and the Design Guidelines from the interviews and the comments.

\section{Quantitative data analysis}


The data from the questionnaires was analysed using boxplots to indicate the overall understandability and usefulness of the SLS Framework, Interaction Model, and Design Guidelines. For the data from the exercises, we counted the number of indicated occurrences (see Table 7.2) of aspects of the Framework, the Interaction Model, and the Design Guidelines.

\section{Exercise 1}

Recognising the SLS elements (components, attributes, and light patterns) that are implemented in the given example (Lungplant).

\section{Exercise 2}

- Recognising the data transformation steps (Processing, Mapping, Combining, and Rendering) and data transformation stages (Processed Data, Abstract Representation, Specified Presentation, and Physical View) in the given example (Time Glow).

- Recognising the interaction loops in the given example (Time Glow).

- Developing new concepts for the given example (Time Glow) by adding new interaction loops.

\section{Exercise 3}

- Describing ideas with the vocabularies (components, attributes, or light patterns) of the Framework.

- $\quad$ Presenting concepts with the layout of the Interaction Model.

- Generating interaction ideas by adding interaction loops.

- Following the Design Guidelines to collect clues, generate ideas, or complete concepts.

Table 7.2. The actual occurrences of the Framework or the Interaction Model in the three exercises.

\section{Qualitative data analysis}

From the data from the comments and interviews a set of 204 relevant quotes have been created. We divided these quotes into three sets which are related to (the use of) the Framework (50 quotes in total), the Interaction Model (81 quotes in total), and the Design Guidelines (73 quotes in total), respectively. Two researchers (including the author) analysed each of these data sets by means of an affinity-diagramming process (Scupin, 2015). In this process, we first sorted all the remarks into four main categories, which are understandability, usefulness, less-usefulness, and potential benefits. Then we defined thematic subcategories in each of the four main categories. These subcategories summarised the most mentioned or most relevant remarks related to the four main categories. Eventually, we developed three tree 
diagrams (see Figure 7.3, Figure 7.4, and Figure 7.5 in the next section) in Mind Node ${ }^{20}$ too visualise an overview of all the categories, subcategories, and quotes.

\subsection{Results}

In this section, we present the results of the evaluation. The results from the questionnaires are illustrated in Figure 7.2, while Table 7.3 lists the actual uses of the Framework and the Interaction Model in the Exercises. The results from the comments and interviews are visualised by three tree diagrams (see Figure 7.3, Figure 7.4, and Figure 7.5), which present all subcategories generated in the qualitative data analysis.

\subsubsection{Results from the Questionnaires}

Figure 7.2 shows the results from the three Questionnaires in three overviews. These three overviews present the average scores for the statements that related to the Framework, the Interaction Model, and the Design Guidelines, respectively.

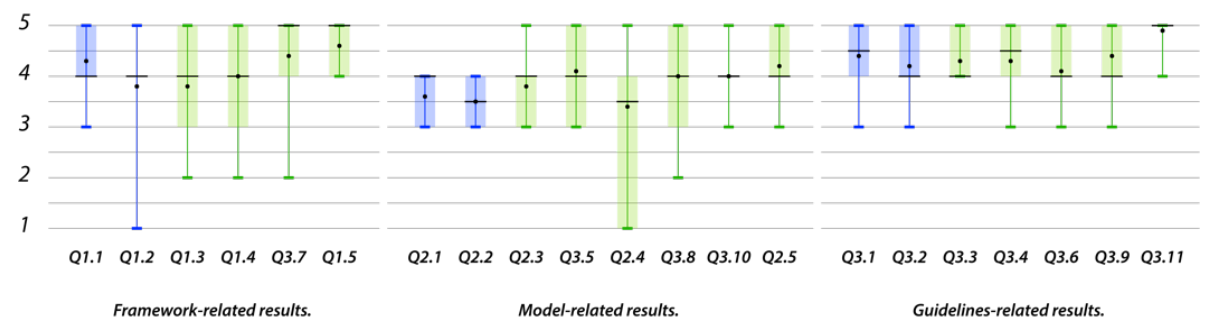

Figure 7.2. An overview of the results from the Questionnaires. The blue boxplots indicate the average rates of the statements about understandability; the green boxplots indicate the average rates of the statements about usefulness; the black lines are median values; the black dots are mean values

\subsubsection{Results from the Exercises}

The results from the exercises are listed in Table 7. 3. The results from Exercise 1 and Exercise 2 show how well the participants understand the Framework and the Interaction Model. Also, there are some results from Exercise 1 and Exercise 2, indicating which parts of the Framework and the Interaction Model are less understandable. The results from Exercise 3 show evidence of using the Framework and the Interaction Model in the hypothetical project.

\footnotetext{
${ }^{20}$ Mind Node. Retrieved on 29.06.2019 from https://mindnode.com/mindnode/mac
} 
Exercise 1.

- All the participants recognised the Active Lights and Active Objects that are implemented in the given example. Also, they correctly identified the Lungplant as Active SLS. However, $30 \%$ of the participants made mistakes in recognising the optional Preset Objects that are implemented in the given example. For instance, describing the textures of the Lungplant's cover as Preset Objects is wrong, because the Preset Objects should be the dark floor where Lungplant is positioned on.

- $30 \%$ of the participants recognised the complete attributes (intensity and rhythm changes in light, and volume change in shape) that are used in the given example. While, the remaining percentage of participants only recognised parts of the used attributes. For example, recognising the attribute of intensity but missing the attribute of rhythm when describing the light attributes.

- All participants were able to recognise the light patterns implemented in the given example. $70 \%$ of them described the light patterns by using the vocabularies from our Framework, such as Sculpted Light or diffused (light) pattern. While, the remaining participants explicitly described the light patterns in their own words. For example, elaborating the light patterns as "transmitting of the shade", which is more detailed than we expected.

Exercise 2.

- In the first exercise, $80 \%$ of the participants mixed up the Abstract Representation and Specified Presentation when describing the design concepts. For example, including the shadow changes into the Abstract Representation is not correct. According to our definition, the shadow changes are part of the Specified Presentation, for they are the outcome of combining light changes and shape changes.

- In the first exercise, only $30 \%$ of the participants were able to completely recognise the right interaction loops in the given example. For the remaining $70 \%$ of the participants, some of them (50\%) only recognised part of the right interaction loops that either alter the Combining step or the Mapping step. Others (40\%) indicated the wrong interaction loop, which is either the one altering the Rendering step or the one altering the Processing step.

- In the second test, every participant was able to recognise parts of the right interaction loops in their own concepts. For example, one proposed a new concept that adapts Time Glow to show the occurrence of bored people in a meeting by the smooth shifting between coloured wall-washed light and coloured shadows. Although she recognised that this interaction alters the Mapping step, she did not recognise that this interaction also alters the Combining step. 
- We collected 17 new concepts in the second exercise. For example, one of the participants developed four concepts by adding four different interaction loops.

\section{Exercise 3.}

- $\quad 90 \%$ of the participants correctly used the components of SLS to describe their ideas. Moreover, $40 \%$ of the participants already did that in the first ideation step (collecting clues) even without the instructions from the Design Guidelines.

- Every participant was able to present his/her clues, ideas, and concepts in the layout of the Interaction Model. However, still 20\% of the participants mixed up the Abstract Representation and the Specified Presentation.

- $70 \%$ of the participants generated interaction ideas by adding interaction loops in this exercise.

- $80 \%$ of the participants followed our Design Guidelines for collecting clues.

- $\quad$ Every participant followed our Design Guidelines for generating ideas.

- Only half of the participants followed our Design Guidelines for completing concepts. What they did concerns detailing the materials, the techniques, and the environment setups to concrete their concepts. The remaining half of the participants only provided little information in the session of completing concepts. However, we also found that they had already mentioned some detailed information that can support realizing their concepts in the session of generating ideas or even in the session of collecting clues.

Table 7.3. An overview of the results of analysing the data from the practices.

\subsubsection{Results from the Comments and Interviews}

By analysing the data from the comments and interviews, multiple subcategories emerged which provide rich specifications of the main categories of understandability, usefulness, less useful, and potential benefits.

In Figure 7.3 and Figure 7.4, we present the subcategories of 'understandability', 'usefulness', and 'less-useful' for the Framework and Interaction Model parts. Also, we present the occurrences of the quotes that are related to the subcategories. For instance, "Complexity $(12 / 7)$ " means that 12 quotes about complexity were found, and that these 12 quotes are provided by 7 participants. Moreover, we show one example quote for each of the subcategories. 

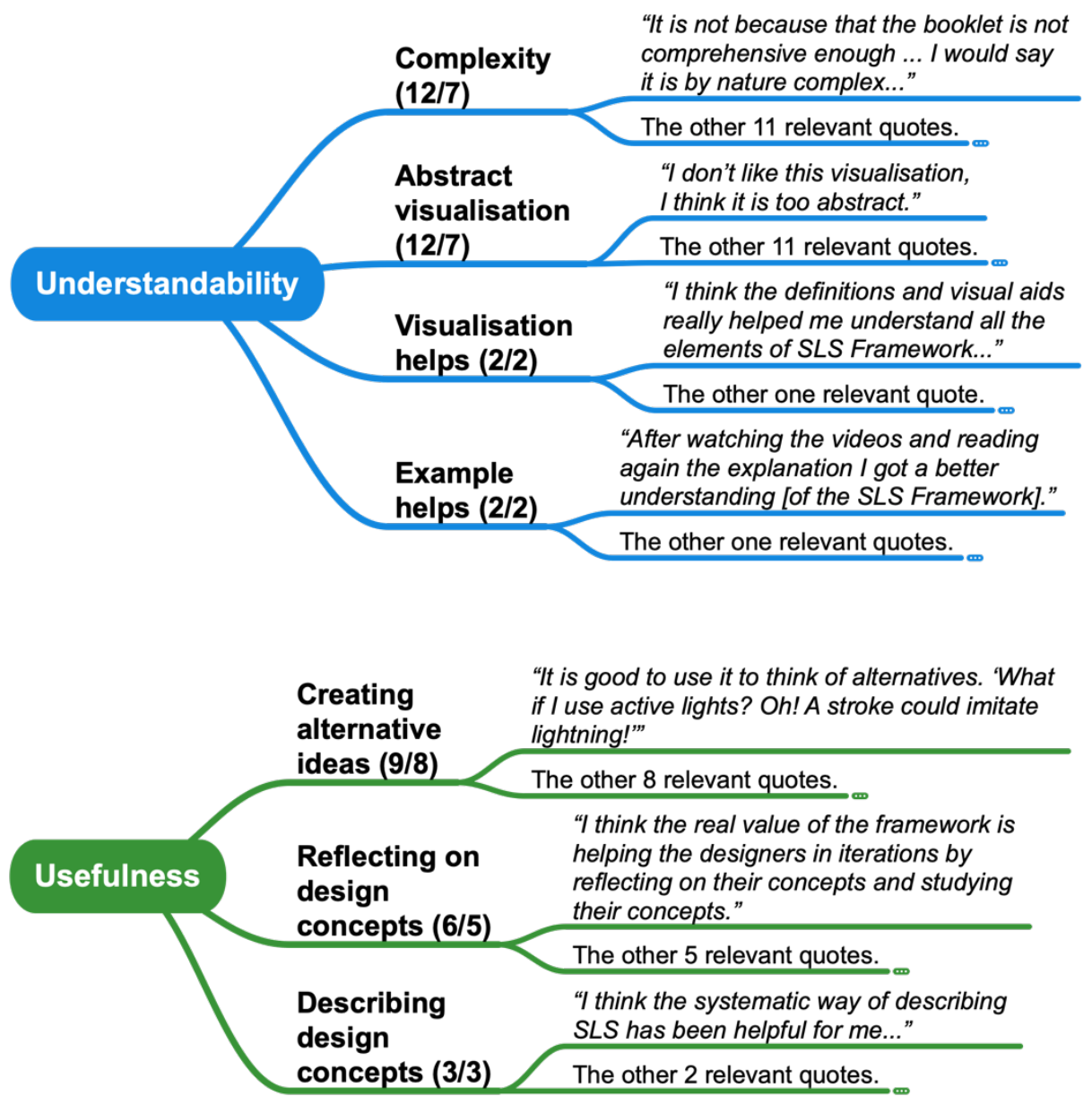

"Perhaps, digital media and tools, for example design card-set, will Less-useful of ready-at-
hand toolkits $(4 / 3)$ help designers easier access to the knowledge."

The other 3 relevant quotes.

Figure 7.3. A tree diagram which presents the results of analysing the qualitative data related to the Framework in the comments and the interviews. 

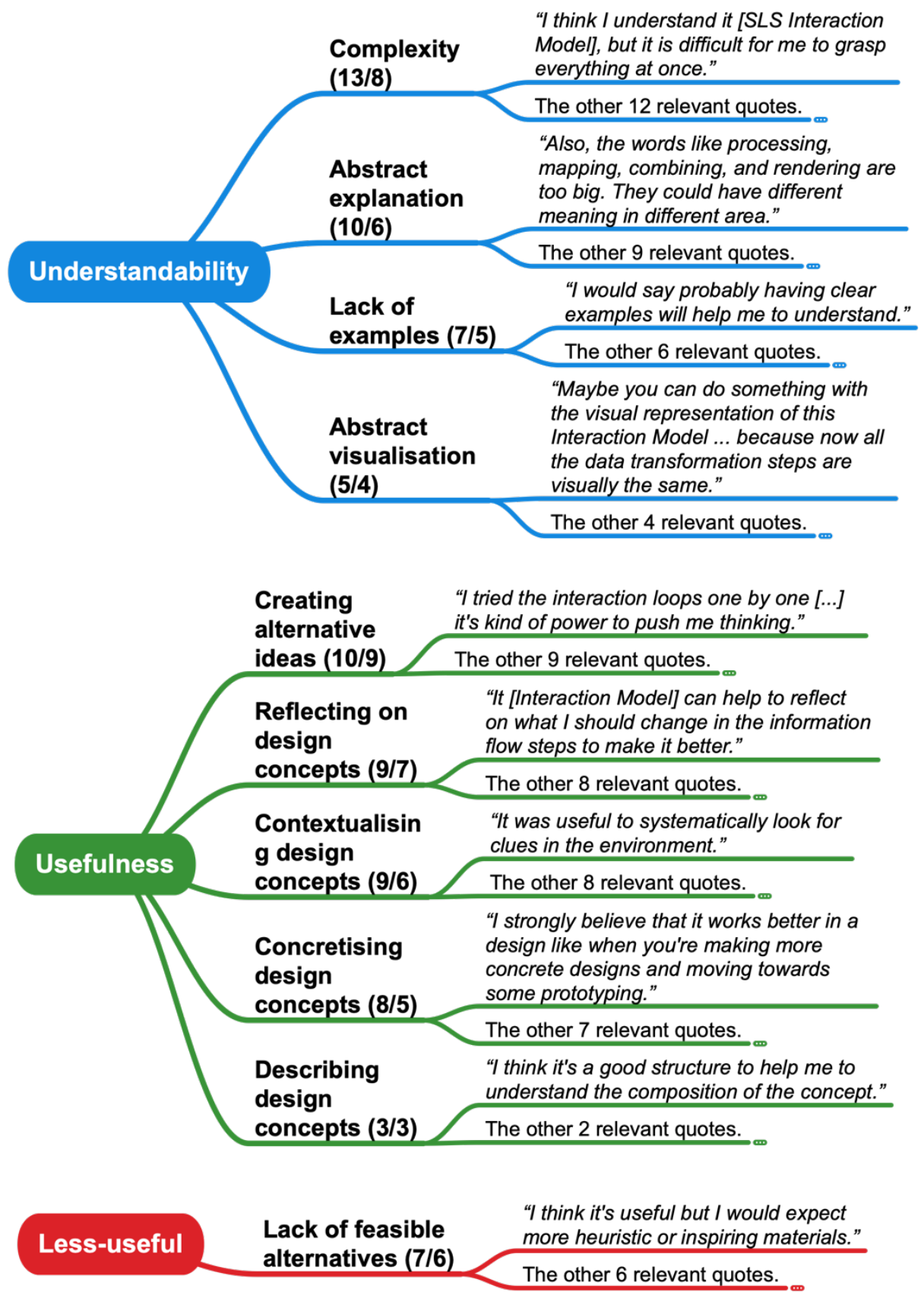

Figure 7.4. A tree diagram which presents the results of analysing the qualitative data related to the Interaction Model in the comments and the interviews. 

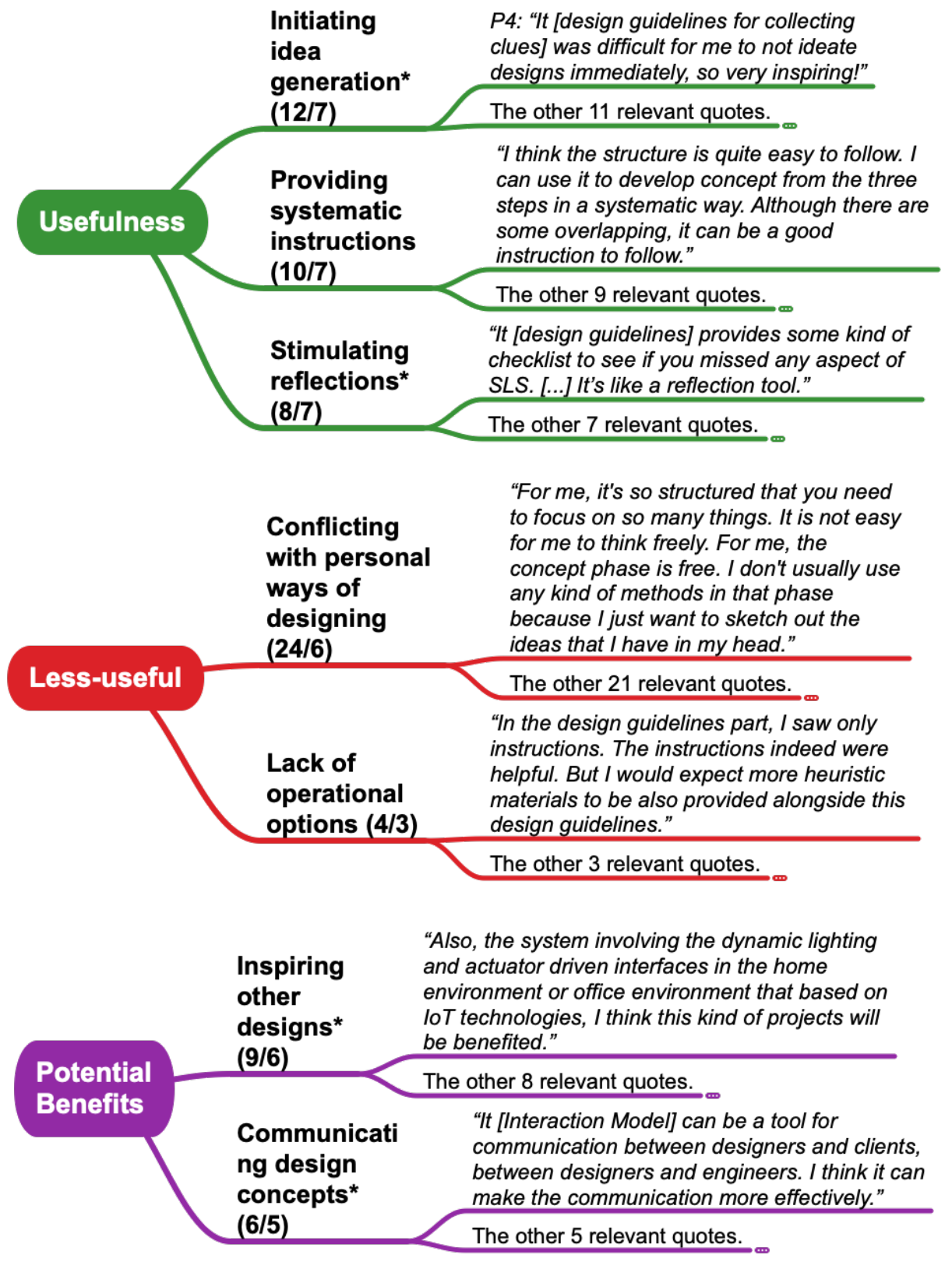

Figure 7.5. A tree diagram which presents the results of analysing the qualitative data related to the Design Guidelines in the comments and the interviews. The subcategories tagged with stars relate to the quality of the Design Guidelines, the Framework, and the Interaction Model as a whole. 
Figure 7.5 presents the subcategories for the Design Guidelines part. These subcategories specify the usefulness, less-useful factors, and the potential benefits of the Design Guidelines. Some of the subcategories relate to the Design Guidelines only, while the others (tagged with stars) also involve the Framework and the Interaction Model as a whole. Notably, the subcategories in the category of potential benefits listed multiple possible applications of the complete knowledge of SLS.

\subsection{Findings}

According to the four goals of the evaluation, we interpret the analysis results from all four sources (questionnaires, practical exercises, comments and interviews) into findings. We first report about the understandability of the Framework and the Interaction Model. Next, we specify the actual use of the Framework and the Interaction Model. Finally, we show the possible improvements and the potential benefits of the SLS knowledge for future situations.

\subsubsection{Understandability}

First, we explain how well the participants understood the Framework and the Interaction Model and what affected the understandability of the Framework and the Interaction Model.

\section{The Framework and the Interaction Model are understandable but complex by nature}

The results from the questionnaires (see blue boxplots in Figure 7.2) show that the Framework and the Interaction Model are understandable in general. The understandability of the Framework is high, while the understandability of the Interaction Model is a bit lower. By checking the results from Exercise 2, we found that many participants were not accustomed to distinguishing the levels of representation and presentation in SLS at the first time of use. This directly affected the ability of the participants to recognise the interaction loops accurately. However, we also found that this issue was alleviated later in Exercise 3 after we provided more explanations in the interviews.

From the interviews, it appears that the most difficult aspect for the participants to understand is the complexity of the knowledge. As one participant summarised: "It is not because that the booklet is not comprehensive enough [...] I would say it [the knowledge of SLS] is by nature complex". On one hand, there are many newly defined elements which took effort for the participants to memorise: "There are too many new words and definitions [in the Framework]", "I needed to reread parts of the text, but it [the Framework] is understandable", and "I think I understand it [the Interaction Model], but it is difficult for me to grasp everything at once". On the other hand, the explanations of the Interaction Model are abstract which caused 
confusions. In particular, there are quite a few participants that mistook the information flow as a sequential design process: "I'm not sure how the sequence of data transformation steps [in the Interaction Model] should be, seems as design steps, or as steps at moment of use". Also, a few confusions were caused by the difficult keywords in the Interaction Model: "The rendering name is confusing because of the analogy to 3D software", and "The process [interaction loops] is more like the intervention from the user or environment".

\section{Concrete examples and effective visual-representations are essential for better understanding the Framework and the Interaction Model}

In this evaluation, we explained the Framework with vivid examples and visual representations. Many participants appreciated that. One participant stated, "I understand it [the Framework], and the videos [examples] were a great help in understanding them [design elements] better", another participant mentioned that, "I think the definitions and visual aids really helped me to understand all the elements of SLS Framework". Still, a few participants expected more effective visual-representations to facilitate better understanding the Framework: "It [visualisation of the Framework] took a lot of back and forth to understand what they mean" and "Some of them [illustrations of the shape attributes] are intuitive, but some are not".

The Interaction Model was introduced mainly with text. In the interviews, many participants expected more examples to embody the data transformation steps (Processing, Mapping, Combining, and Rendering) and the data transformation stages (Abstract Representation, Specified Presentation, and Physical View). One participant stated that "I realised that processing step is about the algorithm. If you mention some relevant examples like mathematics, analysis, or even programming, I think it will be easier for designers to understand", another participant mentioned that "There are some things that are not completely clear to me, like what is specified presentation and what is physical view [...] I would say probably having more clear examples will help me to understand".

\subsubsection{Usefulness}

The Framework and the Interaction Model are intended to support designers in systematically developing SLS concepts. More specifically, we assumed the Framework and the Interaction Model are useful in generating alternative ideas and in reflecting on the concepts. From the results of the questionnaires (see green boxes in Figure 7.2), these assumptions have been validated to some extent. By clustering the usefulness-related subcategories from the qualitative analysis we formulate three findings. 
The Framework and the Interaction Model provide a systematic overview that helps the participants to understand their design concepts better

As one participant stated, "I think they [the Framework and the Interaction Model] helped me to design concepts with an overview by looking at my design at a higher level [...] It's like I was not focusing on one point of the concept but looked through it [...] in a more complete way and from a wider perspective". The overview of the Framework provides vocabularies for participants to recognise what elements were used in their SLS concepts. From the results of Exercise 3, nearly all the participants used the vocabularies in the Framework to describe their ideas. The overview of the Interaction Model provides a structure for participants to specify different data transformation steps and different possible interaction loops in their design concepts. One participant stated that "I think it [the Interaction Model] is a good structure to help me to understand the composition of the concept". Also, we saw that most participants were able to present his/her clues, ideas, and concepts in the layout of our Interaction Model in Exercise 3.

The Framework and the Interaction Model stimulate the participants to create better alternative ideas in an analytical way.

Based on systematic understanding, the Framework and the Interaction Model stimulated participants to create better alternative ideas by analysing the existing concepts and thinking of the other alternative elements in the Framework or the other alternative interactions in the Interaction Model.

In the interviews, many participants considered the Framework as an ideation tool as it provides rich design alternatives: "it [the Framework] gives you more opportunities than when you're thinking by your own". From this, the Framework stimulated the participants to create more alternative ideas in divergent thinking. One participant stated that "You can apply this Framework to help you to explore different alternatives [...] to do diverging [...] I remember that I tried two styles of SLS", another participant mentioned "Every time when I came here [generating ideas] to reflect back, I realised I should have done more ideas". Apart from that, the Framework also stimulated the participants to critique their existing ideas by considering other alternatives in the Framework: "It is good to use it [the Framework] to think of alternatives. What if I use Active Lights? Oh! A stroke could imitate lightning". By doing so, the participants could create reasonably better ideas by comparing the alternatives: "Sometimes three colours might be a bit too much, and it needs to be subtle. Then I started thinking about transitions and different light forms. Am I gonna make it Passive Lights?". 
The results from the exercises show that the Interaction Model is particularly useful in creating interaction alternatives by exploring different possible interaction loops. From the second Exercise 2, we collected 17 new concepts from the participants. In the interviews, one participant explained that "I tried the interaction loops one by one [...] it's kind of power to push me thinking", another mentioned "It [Interaction Model] is very helpful as you can foresee the possible interactions within the system".

Moreover, participants appreciated the Interaction Model as it can raise analytical thinking when generating ideas. From this, the participants believe the Interaction Model can help designers to generate better ideas analytically rather than intuitively. As one participant summarised that "It [Interaction Model] turns the way of intuitive thinking into analytical thinking. And it's really useful because more analytical thinking means the practitioner becomes more reflective in the design process [...] So, he has a higher level of the skills and could make more fundamental innovations in his practice".

\section{The Interaction Model can motivate the participants to develop realistic design concepts in a reflective way}

Apart from creating better ideas, the Interaction Model also motivated some participants to make realistic design concepts, which are more concrete and more contextualised. It is not easy for designers to reach this point in the ideation phase. As one participant stated, "It [Interaction Model] makes the designer step out of the comfort zone and think for himself or herself how I can realise this [concept]".

In the interviews, many participants appreciated the Interaction Model as a checklist which helped them to concretising their design concepts by explicitly adding more details. As one participant stated that "It [Interaction Model] provides some kind of checklist to see if you missed any aspect of SLS", another participant mentioned "It [Interaction Model] reminds you to think about some implicit or ignorable aspects of design [...] to think about the detailed level [...] with multidimensional perspectives. I appreciated that". Also, some participants appreciated the Interaction Model as it provides a structure which helps them to integrate their ideas into concrete concepts: "It [data transformation steps] helps you to consolidate your concept, to convert your different thoughts, to come up with something more concrete and more realistic".

Beyond that, some participants appreciated the Interaction Model as it helped them in contextualising their design concepts. The results from Exercise 3 show that many participants collected clues by following the Design Guidelines, which instruct to explore the design opportunities in the context by using the Interaction Model. In the interviews, one participant 
stated that "I was a bit sceptical, but it was useful to systematically look for clues in the environment [...] I saw there are lamps there and these spotlights are now off on the picture, but they can be on as well. Maybe I can do something with the lamps", and another participant mentioned "it [Physical View] helps you to really think about what the existing environmental factors or artefacts in the given context can be leveraged in your design. It gives an ecological perspective to help the designers to contextualise their preliminary ideas".

\subsubsection{Possible improvements}

By interpreting the subcategories that related to less-usefulness in the qualitative data analysis, we attempt to find possibilities for improving the usefulness of the Framework and the Interaction Model. We derived two findings.

\section{The representations of the Framework and the Interaction Model need to be more accessible}

As we stated before, the Framework and the Interaction Model are too complex to be easily understood at once. That did affect the usefulness of the Framework and the Interaction Model: "If you don't understand it, the Interaction Model will not be helpful". In the interviews, participants gave us multiple suggestions how to improve the accessibility of the Framework and the Interaction Model. Some participants suggested to use simpler visualisation to represent the Framework, "I find the abstract image [visualisation of SLS Framework] not clear. A table with an overview of all options would also help", and more specific illustrations to embody the elements of the Framework, "I recommend using the real effects of the light patterns [...] Maybe you can visualise the design elements with more examples or real $3 D$ sketches instead of $2 \mathrm{D}$ sketches". Also, a few participants advised us to explain the Interaction Model with more examples, "There are some things I was not completely clear about like what is specified presentation and what is physical view [...] I would say having clear examples will help me to understand".

Moreover, a few participants expected us to develop a ready-at-hand toolkit to boost the use of the Framework and the Interaction Model, "I'm thinking, how I can connect two of these things [Framework and Interaction Model]. Probably if I take cards [...] I'm just thinking if I would do it as a recipe cookbook. You have ingredients, and they have to go in there always in same order. First you got the vegetables, then you boil it, then you add sauces. When you serve it, that's a kind of the rendering. It goes in a white plate or black plate depending on the sauce. This is what I was thinking of".

The Design Guidelines provide a systematic overview of what-to-do when using the Framework and the Interaction Model but lack specific how-to-do instructions 
Participants appreciated the Design Guidelines as we provided informative what-to-do instructions using the Framework and the Interaction Model. It can either help beginners to initiate an idea generation process or stimulate experienced designers to reflect on their design concepts. One participant summarises, "I think the Design Guidelines are helpful for getting design [...] For novice designers, they could follow these clearly described steps to perform their practice. For the relatively more experienced designers, they may not have to follow each step of these Design Guidelines, but they can still go through the steps and find something interesting or relevant they want to dig deeper".

However, some participants were expecting specific Design Guidelines about how-to-do when using the Framework and the Interaction Model to develop SLS-concepts. One participant summarised "Basically, I see mostly what to do in the Design Guidelines, but the actual information about how to do it is not given in detail". These alternatives could be the concrete options to realise the data transformation steps: "for the guideline about information sources, you can have different icons to show the possible choices of information sources like, API, IFTTT, and international open source website, etc.". Also, these alternatives could be the suggestions that can help designers to make decisions when developing SLS-concepts: "You indicate the explicit step by step structure. It's good, but maybe in this structure, you can extract some guidelines which can help the designer to build a good design. It means that what if I follow the three steps, but in the end, the design is not good? Is there any golden rule that can make a good design?".

Additionally, we found that some participants complained that the Design Guidelines conflict with their own ways of designing: "Those sequential steps didn't really match with my way of thinking at that moment [...] Personally, I like Frameworks that give me a lot of information [...] I have this information in my head and then I use it in a way I want". We considered this an interesting misconception of the Design Guidelines, as we do not intend to force the participants to follow the Design Guidelines strictly step by step.

\subsubsection{Potential benefits}

In this study, we also collected feedback from the participants about the potential benefits of using the knowledge of SLS. Although these benefits are not validated in this study, they might indicate possible uses of the Framework and the Interaction Model that are worth to be considered in the future.

The Framework and the Interaction Model can support communicating design concepts 
In Exercise 3, the participants were only asked to ideate SLS concepts in a hypothetical project. However, some participants thought the next phases of developing their concepts. In particular, they considered that the Framework and the Interaction Model can help them to communicate design concepts with other stakeholders. For example, to explain design concepts to clients: "it [Interaction Model] can be a tool for communication between designers and clients [...] I think it can make communication more effectively"; to discuss the design concepts with engineers: "I think they are beneficial both for the designers but also the engineers that maybe need to help [...] because the Framework and Interaction Model give them structure to reflect on their ideas on how can they actually make it"; or to help users to specify their requirements to designers: "sometimes the users don't necessarily know what they want. But if they have read something like this [knowledge of SLS] before coming to the designer, they could have an idea as to what the designer could do. And then they can tell the designers what they want".

\section{The Framework and Interaction Model are inspiring for the designs in multiple other related fields}

When discussing the open questions (see Table 7.1) in Interview 3, some participants indicated additional applications of the Framework and the Interaction Model. The most mentioned application is designing interactive art installations: "It's not just only about the lights, it could be used in many other designs as well, but especially when you're doing some interactive and artistic thing in public places". Besides, a few participants considered the Framework and the Interaction Model can inspire the design of lighting interfaces and even smart environments through IoT (Internet of Things) technology. One participant stated that "It's not to build one SLS like we did in the exercise, but it's more to use the elements to think of ways of showing information within an interface. I think that's where I would use it", another participant mentioned that "a system involving dynamic lighting and actuator driven interfaces in the home environment or office environment that is based on IoT technologies, I think this kind of project would benefit".

As a matter of fact, in Interview 3, one participant was inspired by SLS and got an idea of her ongoing project about behaviour change. She stated that: "We're collecting information as well as we want to give information [...] I can easily see how a shape-changing light just using the movement information from the sensor can show whether or not they met their target for the day [...] Maybe we could have a lamp, they need change the shape of some lighting object, whenever they've not met their targets something that's tangible that they can feel that they can push, it's possible. You give me ideas!" 


\subsection{Discussion and Conclusion}

In this chapter, we have presented the evaluation of the SLS Framework and the Interaction Model. In this evaluation, ten experts reviewed and used the Framework and the Interaction Model to develop SLS concepts in a hypothetical project. We collected quantitative data from questionnaires and practical exercises, and qualitative data from comments and interviews. In the end, we interpreted the quantitative results and qualitative results into four clusters of findings in line with the four goals of the evaluation. Now we will discuss the limitations of the study. We will end this chapter with a conclusion of the evaluation and some recommendations for future studies.

\subsubsection{Limitations of the study}

In this study, we designed a 55-page booklet to present all SLS knowledge. We tried to clarify the content as compact as possible to reduce the efforts for the participants to learn the SLS knowledge. We also put effort in presenting it in an attractive manner, indicating the relevance for the design practitioners. We still received complaints that it took too much effort to grasp all the knowledge at once in a short period of time. This influenced the understandability and the usefulness of the Framework and the Interaction Model. However, we note that the results of this evaluation only reflect the usefulness of the Framework and the Interaction Model at the first time of use.

The usefulness of the Framework and the Interaction Model were only validated in the context of a hypothetical SLS project. Strictly speaking, this validation might not be convincing enough if compared to a situation where the Framework and the Interaction Model were offered to designers in a real SLS design projects. Although the experts that participated in the study have both backgrounds in design and in educating design students, it remains uncertain how useful the Framework and the Interaction Model would be for designers that have no research background.

\subsubsection{Conclusions from the study}

By reflecting on the first two goals of the evaluation, we conclude the understandability and the usefulness of the Framework, the Interaction Model, and the Design Guidelines as follows.

\section{Framework}


The Framework is considered to be complex by nature because it consists of many newly defined elements. However, it is understandable to a high level. This is accomplished by the rich examples and visual-representation alongside the text explanations in the booklet.

The Framework provides a systematic overview, which can stimulate designers to create better alternative ideas for SLS in a more analytical way. Firstly, the Framework provides vocabularies for designers to describe their ideas systematically. Additionally, such systematic descriptions can help designers to be aware of the other possible alternatives in the Framework. Finally, designers can reflect on their existing ideas and create better alternative ideas.

\section{Interaction Model}

The Interaction Model is understandable but requires more explanation than we expected. The elements of Abstract Representation and Specified Presentation are especially complicated and cannot be easily understood. It seems participants were not used to distinguish the levels of representation and presentation when designing SLS.

The Interaction Model also provides a systematic overview for designers to understand their SLS concepts better. More specifically, this overview can help designers to analyse their existing concepts by recognising different data transformation steps and different possible interaction loops. By reflecting in this way, designers can create reasonably better alternative ideas or concretise their existing ones. Also, the Interaction Model can support designers discovering the opportunities of contextualising their design concepts by using the environment to compose their concepts or adding appropriate possible interaction loops.

\section{Design Guidelines}

The Design Guidelines provide a systematic approach for designers to use the Framework and the Interaction Model in the ideation process. Firstly, the Design Guidelines for collecting clues can support designers contextualising their design concepts by using the Interaction Model to explore the design opportunities in the design context. Secondly, the Design Guidelines for generating ideas can stimulate designers to create alternative ideas by using both the Framework and the Interaction Model to analyse their existing ideas. Finally, the Design Guidelines for completing concepts can motivate designers to concretise their design concepts by using the Interaction Model to reflect on the missing aspects of their existing concepts.

\subsubsection{Recommendations for further development}


Firstly, the representations of the Framework and the Interaction Model need to be improved. More specifically, the visualisation of the Framework needs to be optimised by applying more specific illustrations (e.g., three-dimensional illustrations) and more effective visualisations (e.g., simple diagrams). Also, the data transformation steps and data transformation stages in the Interaction Model needs to be explained better with more concrete examples.

Secondly, developing ready-at-hand toolkits will enhance the usefulness of the Framework and the Interaction Model. In this study, we provided a booklet for the participants to read and learn knowledge about SLS. It turned out that participants did consult and recap the information, which can be made more efficient. Therefore, we expect toolkits (e.g., card-sets) that enable designers to access the Framework and the Interaction Model as a quick source of reference or guidance during design actions can be very effective. Moreover, we believe that such toolkits can also support designers to efficiently presenting their SLS concepts when communicating with co-designers or other stakeholders.

Finally, providing how-to-do suggestions in the Design Guidelines can improve the usefulness of the Framework and the Interaction Model. However, this requires more concrete examples of SLS to embody the abstract elements in the Framework and the Interaction Model. In the future, we would like to promote the knowledge of SLS in broader design spaces (e.g., interactive art installations, intelligent lighting interfaces, IoT-based smart environments, etc.) to gain more experience of designing SLS. By that time, we believe that a set of more operational SLS Design Guidelines will emerge. 


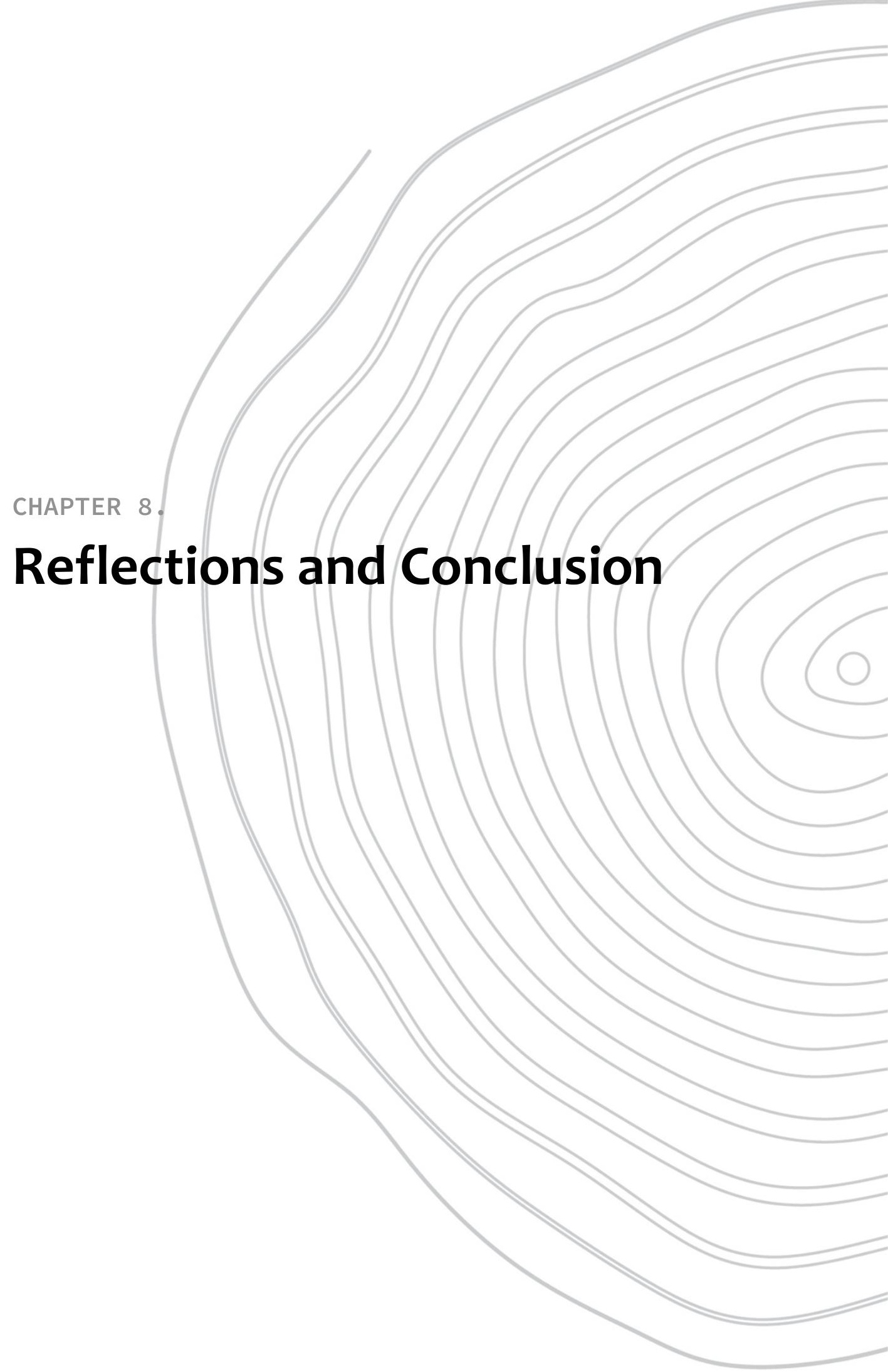


At the end of this dissertation, we summarise our contributions by reflecting on our design experience and the research questions that we posed in the introduction chapter. Also, we discuss the limitations of the work presented in this dissertation, as well as possible directions for future work.

\subsection{Contributions of This Dissertation}

The primary goal of the work presented in this dissertation was to provide design-relevant knowledge that can support the design of current and future ambient light systems, which combine light with shape-changing. We named such lighting systems Sculpting Light Systems (SLS). To achieve this goal, we carried out a hands-on design activity, from which we defined three specific research questions. In this section, we will reflect on our approach and each of the three research questions to summarise the contributions of this dissertation.

\subsubsection{The design space of SLS}

Our first research question addresses an understanding of SLS. How can we define and understand the design space of SLS? What are the design challenges for SLS? And if we are aware of these challenges and acquired an understanding, how can we present this knowledge in a systematic way? In this dissertation, we introduced the novel concept of Sculpting Light Systems (SLS), which combines light with shape-changing to produce meaningful light patterns for Information Decoration. This approach follows the principle of Calm Technology and provides a desirable alternative to the use of direct light in the design of future ambient light systems. In particular, we have motivated the idea of SLS by considering the increasing complaints of people about "information overload" with the development of Ubiquitous Computing. The concept of SLS indicates a new design space, in which light sources are integrated in their physical environment. To explore this new design space, we have developed four prototypes (chapter 2 and chapter 4) and reviewed a collection of state-of-art examples of SLS (chapter 3).

As a result, we acquired an initial understanding of SLS which led to the formulation of six design challenges for developing SLS and a SLS Framework. Now we reflect on this initial understanding by summarising the insights we have gained from dealing with the design challenges and using the SLS Framework in the studies presented in this dissertation.

\section{Design considerations for developing SLS}

SLS are more complex than conventional ambient light systems, which mainly focus on the direct manipulation of light while the features of the physical media are rarely considered. 
Developing SLS challenges designers to take full advantage of physicality by combining the natural qualities of light and materials to create aesthetic, meaningful, and environmentally appropriate ambient light systems. In chapter 2, we have introduced six design challenges for developing SLS based on our initial design explorations. In our later design explorations (in chapter 4) and concept generations (in chapter 5 and chapter 6), we have presented different approaches to tackling the design challenges. By reflecting on these experiences, we are able to express three design considerations for developing SLS.

\section{Creating aesthetic and meaningful light patterns}

SLS combine light with shape-changing to produce light patterns, which play a key role in information presentations. However, in design practice, the light patterns in SLS are not always predictable, or, at least not easy to be foreseen precisely compared to pixelated direct light in the case of conventional ambient light systems. Therefore, trying out combinations of light and different materials is essential when developing SLS. Based on our experiences, it was enjoyable to play with light but expensive to get materials that are appropriate for building kinetic structures and for producing light patterns. There is a trade-off between deformability (e.g., elasticity) and optical properties.

An alternative way of experimenting with light patterns is employing computer simulation. In chapter 5, we have presented multiple SLS concepts that were developed with the aid of Rhinoceros (for modelling the physical shape-changing of SLS) and Keyshot (for rendering the light patterns of SLS). In this way, we can easily combine different forms of shape-changing and light settings. Also, we can preview dynamic light patterns in real time.

\section{Actualising subtle shape-changing}

As we mentioned above, it is feasible to simulate a dynamic shape-changing performance in the computer. For instance, Grasshopper 3D (a parametric plug-in for Rhinoceros) supported us in designing the shape-changing of the lampshade shown in chapter 5. However, it remains a challenge to bring the conceptual shape-changing into reality. In particular, it is not easy to implement the shape-changing in SLS which is not only visually subtle but also quiet in terms of (mechanical) sound production. This is due to the fact that techniques of shape-changing actuation are still under-developed, and it is challenging for most types of actuators to perform shape-changing without noise. Although there are a few actuation methods that can perform shape-changing quietly, their restricted controllability limits the information they can represent. For example, there is always a delay for the shape-changing actuated by smart materials (e.g., shape-memory wires); also, it is hard to avoid the undesirably visible vibration in the dynamic shape-changing actuated by electromagnets. 
The compromise to create subtle shape-changing is building a complex and fine structure, which combines the light fixtures, shape-changing actuators (e.g., motors), connection mechanisms, and electronics (e.g., sensors) ingeniously. It is more difficult than designing a conventional ambient light system, which only concerns the light settings. More specifically, different kinds of expertise might be involved to achieve such a complex system, such as mechanical design, material design, lighting design, computer programming, electronic design, and so on. In this sense, the knowledge embedded in the SLS Framework and SLS Interaction Model provides an alternative basis for different expertise to communicate either design concepts or design requirements in the design process.

\section{Seamless Integration of SLS into the user's context}

SLS are meant to be seamlessly integrated into the user's environment so that he/she can perceive the information display subtly without being overburdened. According to our design experiences and user tests presented in chapter 2, we found three design challenges. The first one concerns the uncontrollable environmental light which might impact the visibility of the subtle light patterns. For example, it is not easy to implement SLS within an environment in which there is a continuous change of the daylight. The second challenge concerns the set-up of surroundings of SLS to facilitate well-integrated ambient displays. This design challenge requires designers to consider SLS as a part of the environment rather than an independent information display system. The third design challenge is about exploiting the tangibility of shape-changing to support natural interactions for the user to manipulate ambient displays. Although tangible interactions are not essential for developing SLS, according to the user feedback on Timer Shade (section 2.4.4), it possibly can help people to learn and better understand the ambient displays of SLS.

\section{A framework for SLS}

In this study, we proposed a SLS Framework (section 4.4), which outlines the design space of SLS with a set of design alternatives at two levels. Firstly, it presents a systematic overview of the alternative building blocks of SLS: five basic components for construction, three types of attributes for manipulation, and three kinds of light patterns for information mapping. Secondly, it contains diverse alternative design options for implementing these building blocks. These options include: different kinds of light sources and physical objects for the implementation of SLS components (section 3.2.2), various properties for defining the performances of light change and shape change (section 3.2.4), and six ways of deploying light sources and physical objects for creating light patterns (section 3.2.5). By reflecting on the use of the SLS Framework by ourselves (chapter 5 and chapter 6 ) and by our participants (chapter 
7) in the study, we conclude two potential ways of applying the SLS Framework to enrich the design space of SLS in the future.

\section{Stimulating creations in developing SLS-concepts}

In the design area, the Framework provides rich alternatives for designers to explore diverse possibilities of implementing SLS in the concept design stage. Besides, it provides vocabularies for designers to describe their ideas in a systematic way, by which they can be aware of the other possible alternatives in the Framework. Therefore, designers can be stimulated to create better alternative ideas from comparing and critiquing their existing ideas. Additionally, we are expecting designers to develop more creative SLS-concepts by exploring the design alternatives which are not elaborated or even involved in the current Framework yet. From this, the SLS Framework can be more useful with not only more specific but also more new design alternatives to support the design of SLS in the future.

\section{Supporting concrete studies on SLS}

In the research field, the Framework provides a fundamental basis, on which researchers can discuss open research questions of SLS in the future studies. One of the potential studies would be a literature review, in which researchers can use our Framework to analyse the existing work of SLS in order to understand the development status of SLS. For example, what kind of design alternatives of SLS are applied widely, for what reasons are certain design alternatives applied less, and what design alternatives are missed in the SLS Framework presented in this dissertation? By answering these questions, we are expecting deeper understandings of the design space of SLS. This understanding will support creative activities in developing SLS as well as the SLS Framework and the design space of SLS.

\subsubsection{Systematic approach to designing SLS}

In this study, although we have presented an initial understanding of the design space of SLS, the Framework is still too broad to support designing SLS in a specific context. That's why we formulated our second research question: can we propose an interaction model that integrates the design knowledge of understanding SLS in existing design processes? This question calls for means to apply insights in SLS to opportunities of designing SLS for Information Decoration. As a result, we formulated the SLS Interaction Model, based on existing interaction models from communication science and information visualization. The SLS Interaction Model provides designers with a coherent picture of what is possible when developing an adaptive SLS for Information Decoration. The Interaction Model consists of an information flow and four means of interaction loops. The information flow (section 5.2) 
elaborates four data transformations steps within SLS from raw data to physical view. While the interaction loops (section 5.3.1) open up the possibilities of adapting SLS to the user's context by altering the four data transformation steps. These interaction loops could be triggered either from the user side or from the environmental side.

The Interaction Model can be used as a reflection tool for designers to understand their design concepts in a detailed level. More specifically, the Interaction Model can help designers to analyse their existing concepts by recognising different data transformation steps and different possible interaction loops. By reflecting on these elements, designers can create better alternative ideas or enrich existing ideas with more details.

Both the Framework and the Interaction Model provide designers with so-called 'intermediate-level design knowledge' (Höök \& Löwgren, 2012). On the one hand, the knowledge supports an understanding of the design space of SLS which is more generic than the insights gained from the design explorations. On the other hand, we only validated the knowledge in the ideation phase of developing SLS. Therefore, neither the Framework nor the Interaction Model is ready to be applied for supporting the entire design process of SLS. However, we consider the Framework and the Interaction Model as important contributions in creating a better understanding of the design space of SLS and a systematic approach to the design of SLS. Extensive research on the use of the Framework and the Interaction Model in more design practices of SLS will further contribute to this understanding, and may also lead to a comprehensive approach to support designers in the entire process of developing SLS for Information Decoration in the future.

\subsubsection{Design guidelines for SLS}

Our last research question relates to how this new knowledge can be made operational in realistic design process. To answer this question, we introduced a set of design guidelines (see chapter 6), which propose an alternative approach to help designers to systematically generate plausible SLS concepts by applying the SLS Framework and Interaction Model. More specifically, they deploy the considerations of the different design elements presented by the Framework and the different design aspects presented by the Interaction Model into three ideation sessions, which are collecting clues (section 6.3.1), generating ideas (section 6.3.2), and completing concepts (section 6.3.3).

The design guidelines are not intended to be a part of the body of 'intermediate-level design knowledge' but can be seen as a research tool, which allowed us to evaluate the usefulness of the Framework and the Interaction Model. Apart from that, the design guidelines also helped 
us to explore what knowledge or tools are possibly still needed in supporting designers better using the Framework and the Interaction Model. For example, one of our participants was thinking that (see section 7.4.3) the Framework and the Interaction Model could be integrated as a "recipe cookbook", in which the design elements of SLS are presented by cards. By playing with these cards, the designers can develop SLS concepts with more fun.

\subsection{Limitations}

The main contributions of this dissertation are the design-relevant knowledge that aims to support the design of SLS in a systematic way. By reflecting on the generation, validation, and application of the knowledge, we discuss the limitations of the work presented in this dissertation.

The review of the state-of-the-art examples played a core role in generating the Framework. It comes with the limitations in the completeness of the sample for analysis. First of all, it is difficult to collect a complete set of examples in the art and design area by just searching relevant keywords, which is an effective way for literature review in research though. On the one hand, the description of the design projects or artistic work can be very subjective and not include the keywords we used. On the other hand, we could have missed some examples that are not described online in English. Second, there are many alternative platforms offline for the designers or artists to present their work. For instance, we did see several relevant examples of SLS in art exhibitions. For this type of relevant work, it is hard to collect and compile them in the review from which we generated the Framework.

In our user study, we only validated the usefulness of the Framework and the Interaction Model in a hypothetical project. There's no doubt that the results will be more convincing if we offered the knowledge to the designers in a real project. However, developing SLS in practice is time-consuming and costly, which is one of the design challenges we have presented in section 8.1.1. Therefore, as a first step, we considered that it would be costeffective to evaluate their usefulness in ideating SLS first. While a hypothetical project is enough to do so. According to the results from our evaluations, it turned out that there indeed will be more work (section 8.3.1) to be done before the Framework and the Interaction Model can support the design of SLS in a real project.

The knowledge we presented in this dissertation aims to support designing SLS for Information Decoration. Both the SLS and the Information Decoration are very new concepts that are lacking specific application examples. Although we have presented several design cases in this dissertation (see chapter 2 , chapter 4 , and the design concepts shown in chapter 
5 and chapter 6), they are mostly conceptual and not formally validated in practice. Therefore, we propose that more applications of designing SLS for Information Decoration are needed to contribute to further consolidate the usefulness of the knowledge we presented in this dissertation.

\subsection{Future Work}

In this dissertation, we have explored the design space of SLS. The resulting knowledge proved to be useful as it provides a systematic overview that helps designers to understand and ideate SLS concepts better. However, how to apply the knowledge in real projects and what knowledge is still needed for specific applications are questions that need further explorations. To answer these questions, we propose three possible directions for future work.

\subsubsection{Developing an accessible toolset}

The Framework and the Interaction Model can help designers to systematically develop SLS concepts. However, they both deploy rather complex knowledge which might affect their usability. Therefore, we consider that developing an accessible toolset based on the knowledge will be necessary to enhance the usability of the Framework and the Interaction Model in practice. Such an accessible toolset could be an interactive digital platform, which supports designers learning and checking the knowledge of SLS within a graphic interface. For example, representing the Framework and the Interaction Model with different layers of information (e.g., element, definition, and examples, etc.) by means of mind map. By this digital mind map, designers can always have an overview of the possibilities of designing SLS, also they can check or hide the detailed information by touching the screen easily. Moreover, the accessible toolset could be physical to support playful activities during brainstorming in a group setting. For example, representing the design elements of SLS with card sets, each of which contains the definition of one design element on the front side and some heuristic examples on the back side.

\subsubsection{Generating context-specific insights}

The knowledge we presented in this dissertation provides designers with a systematic overview of the design elements of SLS by the Framework and the Interaction Model. These design elements can support designers in ideating SLS-concepts with many possibilities. But we also notice that the operational insights to support designers in implementing SLS in a certain specific context are still missing. To fill in this gap, we are expecting the practitioners 
to generate context-specific insights to enrich the understanding of the design space of SLS in the future. For example, we can foresee that the implementations of the pre-set objects in the home context and public space can be very different. For the home context, the decorative wallpaper naturally provides a pre-set object, which is so familiar to the user that he/she is able to understand the meaningful light projections cast on it. While, for the public space, using a simple white wall as pre-set object to show the meaningful light projections can be necessary to guarantee that everyone can perceive and interpret the information displays everywhere in the space. For another example, sculpted light patterns could be suitable in the context (e.g., retail shop) where SLS are implemented to draw the user's attention gently. The projected light patterns could seamlessly fit in a quiet environment, such as museum, library, sanatorium, etc.

To generate such insights as mentioned above, practitioners need to evaluate and understand what the characteristics of the design elements of SLS would be in different contexts. Based on Pousman and Stasko's evaluation approach of ambient information systems (Pousman \& Stasko, 2006), we recommend the practitioners to systematically generate context-specific insights of how to deploy the design elements of SLS in the aspects of information capacity, notification level, representational fidelity, and aesthetic emphasis.

\subsubsection{Inspiring other related fields}

The research presented in this dissertation mainly focuses on exploring the design space of SLS that are particularly implemented for Information Decoration. As a matter of fact, there are many other applications for SLS than conveying information. One of the most typical applications is about (interactive) light installations, which are meant to create aesthetic (and interactive) experiences of light. Examples can be seen from the review of state-of-the-art examples (see Appendix I) we have presented in section 3.2. Hence, we believe that the knowledge of SLS can challenge designers and artists to explore more innovative work beyond the ones that can be described by the SLS Framework. For instance, what if we create a light installation by means of diffraction, which is not included in the SLS Framework yet. Also, besides considering the five basic components of SLS, it is possible to create fantastic light installations by taking the user's changing viewing angles into account. An example of that is Anamorphic Mirror ${ }^{21}$. In this case, the user can only perceive a reflected logo when he/she is standing at a specific position in front of a facetted mirror. Inspired by that, we think that there could be two new components that can complement the SLS Framework in the future. One is

\footnotetext{
${ }^{21}$ ART+COM Studios (2011). Anamorphic Mirror. Retrieved from https://artcom.de/en/project/anamorphic-mirror/
} 
an active user who perceives the SLS in motion, such as people who are walking, cycling, driving, and etc. The other is a passive user who perceives the SLS with a relevantly constant viewing angle. For example, people working in front of a desk, lecturing on a stage, standing guard beside an entrance, etc.

The concept of SLS blurs the boundary between the design space of lighting interfaces and shape-changing interfaces. Although currently, SLS is avant-garde, we believe that it can stimulate practitioners to explore more novel lighting or shape-changing interfaces in the near future.

Firstly, SLS can derive a new form of lighting interfaces, in which shape-changing is implemented as either output for producing light effects or input for lighting controls. This interaction can be more intuitive than touching a screen or manipulating an independent tangible controller in the cases of traditional lighting interfaces. For example, the Timer Shade we presented in section 2.4 could also be implemented in a home context as a decorative and interactive lighting, which enables the user to lighten or dim the light by pulling down/up its deformable coiled cap.

Secondly, SLS can broaden the design space of shape-changing interfaces by allowing the light representations to be positioned at the surroundings of the shape-changing objects. For conventional shape-changing interfaces, light is implemented to augment the shape change through emitting or projecting. Such interfaces are independent to the environment as their information displays are spatially restricted on the body of the shape-changing objects. To seamlessly integrate the shape-changing interfaces into their environment in the future, we encourage the shape-changing designers to take full advantage of the nature of light rather than deploying the light just as a shining "skin" of the shape-changing objects. For example, the reflected light projections of Reflective Clock (section 4.2) have showcased the potential of augmenting shape-changing displays with light patterns, which are environmentally appreciated for changing colour according to the colour of the sticky-note where light projections shine on.

In addition, I believe the insights presented in this thesis can inspire other researchers who are working on areas that combine art and technology just like the concept of Information Decoration does. Nowadays, more and more artists and designers are attempting to integrate art forms with new technologies, such as Artificial Intelligence, Augmented Reality, Big Data, Internet of Things, etc. However, the relevant knowledge in these fields is often too technical for designers to apply in practice. There is a need for knowledge that can help designers to systematically understand the domain where art and new technologies are integrated. It's like learning a new language which starts with vocabularies and then syntax. The vocabularies 
present basic design elements as our Framework does. These design elements can help designers to be aware of rich alternatives in creation. The syntax provides systematic approach as our Interaction Model does. This systematic approach can support designers in communicating ideas with engineers and even scientist more easily.

\subsection{Conclusions}

In this thesis, we have explored the design space of SLS, which combines shape-changing techniques with light to pursue lighting systems as ambient displays featured with Information Decoration. We have gained an understanding of such design space by two design explorations, as well as a review of state-of-art examples of SLS. This enabled us to formulate a Framework for SLS (section 4.4). We also proposed an Interaction Model of SLS to support designers in designing SLS for adaptive Information Decoration, based on an information flow (section 5.2.2) and four possible means of interaction loops (section 5.3.1). Finally, we integrated the use of the SLS Framework and the SLS Interaction Model into a set of design guidelines to support designers in developing SLS specifically in the ideation phases (section 6.3). This design-relevant knowledge is useful in ideating SLS-concepts. Moreover, it can inspire design in the fields of interactive light installations, lighting interfaces, and shapechanging interfaces.

This dissertation is just one small steppingstone on a long journey towards the vision of calm technology. We hope to see more applications of SLS that are seamlessly integrated in our environment in the future. At that moment, we believe everybody can benefit from SLS that are informative, aesthetic and subtle in the age of an information explosion. 



\section{References}

Alexander, J., Weichel, C., Hardy, J., Vidler, J., \& Taher, F. (2015). ShapeClip. Proceedings of the 33rd Annual ACM Conference on Human Factors in Computing Systems (CHI '15), 19-28. https://doi.org/10.1145/2702123.2702599

Card, S. K., Mackinlay, J., \& Shneiderman, B. (1999). Departments-Book Preview-Readings in Information Visualization: Using Vision to Think. In Interactions (Vol. 6, p. 51).

Carpendale, M. S. T. (1999). A Framework for Elastic Presentation Space. Dept. of Computer Science. Simon Fraser University. https://doi.org/http://doi.acm.org/10.1145/932254

Cha, S., Lee, M.-H., \& Nam, T.-J. (2016). Gleamy. Proceedings of the TEI '16: Tenth International Conference on Tangible, Embedded, and Embodied Interaction - TEI '16, 304-307. https://doi.org/10.1145/2839462.2839501

Coelho, M., \& Zigelbaum, J. (2011). Shape-changing interfaces. Personal and Ubiquitous Computing, 15(2), 161-173. https://doi.org/10.1007/s00779-010-0311-y

Dahley, A., Wisneski, C., \& Ishii, H. (2003). Water lamp and pinwheels. In CHI 98 Cconference Summary on Human Factors in Computing Systems (pp. 269-270). https://doi.org/10.1145/286498.286750

Dalton, N. (2013). TapTiles: LED-based floor interaction. Proceedings of the 2013 ACM International Conference on Interactive Tabletops and Surfaces, 165-174.

Diaz-Marino, R., Marquardt, N., Greenberg, S., Ballendat, T., \& Wang, M. (2011). Proxemic interactions. Interactions, 18(1), 42. https://doi.org/10.1145/1897239.1897250

Ed Huai-Hsin Chi, \& Riedl, J. T. (2002). An operator interaction framework for visualization systems. In Proceedings IEEE Symposium on Information Visualization (Cat. No. 98TB100258) (pp. 63-70). https://doi.org/10.1109/infvis.1998.729560

Eggen, B., \& Van Mensvoort, K. (2009). Making Sense of What Is Going on 'Around': Designing Environmental Awareness Information Displays. In Awareness Systems (pp. 99-124). https://doi.org/10.1007/978-1-84882-477-5_4

Franchin, G., Yang, M., Stern, M., Kayser, M., Oxman, N., Houk, P., ... Dave, S. (2016). Additive Manufacturing of Optically Transparent Glass. 3D Printing and Additive Manufacturing, 2(3), 92-105. https://doi.org/10.1089/3dp.2015.0021

Frisken-Gibson, S. F., Bach-Y-Rita, P., Tompkins, W. J., \& Webster, J. G. (1987). A 64Solenoid, Four-Level Fingertip Search Display for the Blind. IEEE Transactions on Biomedical

Engineering,

$B M E-34(12)$, 963-965. 
https://doi.org/10.1109/TBME.1987.325937

Gooch, J. W. (2011). Lumen. In Encyclopedic Dictionary of Polymers (pp. 435-435). https://doi.org/10.1007/978-1-4419-6247-8_7062

Goulthorpe, M., Burry, M., \& Dunlop, G. (2001). Aegis hyposurface: The bordering of university and practice. Proc. of ACADIA, (March 2001), 344-349. Retrieved from http://cumincad.architexturez.net/system/files/pdf/811d.content.pdf

Höök, K., \& Löwgren, J. (2012). Strong concepts. ACM Transactions on Computer-Human Interaction, 19(3), 1-18. https://doi.org/10.1145/2362364.2362371

Hornecker, E., Gielen, J., Hartmann, J., Dondera, F., Bornemann, M., Schmandt, M., ... Fischer, P. T. (2015). Exploring the Potential of Depictions with Sun Reflections. In Proceedings of the 4th International Symposium on Pervasive Displays (pp. 217-224). https://doi.org/10.1145/2757710.2757712

Ishii, H. (2004). Tangible bits. Proceedings of the ACM SIGCHI Conference on Human Factors in Computing Systems, 3. https://doi.org/10.1145/604045.604048

Ishii, H., \& Ullmer, B. (2012). Tangible User Interfaces (pp. 465-490). https://doi.org/10.1201/b11963-25

Iwata, H., Yano, H., Nakaizumi, F., \& Kawamura, R. (2005). Project FEELEX. Proceedings of SIGGRAPH 2001, 469-476. https://doi.org/10.1145/383259.383314

Jansen, Y., \& Dragicevic, P. (2013). An interaction model for visualizations beyond the desktop. IEEE Transactions on Visualization and Computer Graphics. https://doi.org/10.1109/TVCG.2013.134

Jansen, Y., Hornbæk, K., Karnik, A., Alexander, J., Subramanian, S., Isenberg, P., ... Dragicevic, P. (2015). Opportunities and Challenges for Data Physicalization. In Proceedings of the 33rd Annual ACM Conference on Human Factors in Computing Systems (pp. 3227-3236). https://doi.org/10.1145/2702123.2702180

Jung, H., Altieri, Y. L., \& Bardzell, J. (2010). SKIN: Designing Aesthetic Interactive Surfaces. In Proceedings of the fourth international conference on Tangible, embedded, and embodied interaction (pp. 85-92). https://doi.org/10.1145/1709886.1709903

Kaila, L., Raula, H., Valtonen, M., \& Palovuori, K. (2012). Living wood: a self-hiding calm user interface. In Proceeding of the 16th International Academic MindTrek Conference (pp. 267-274). Retrieved from https://dl.acm.org/citation.cfm?id=2393191

Kim, S., Paulos, E., \& Gross, M. D. (2010). WearAir. In Proc. TEI '10 (p. 295). https://doi.org/10.1145/1709886.1709949

Kyung, K. U., Lim, J. M., Lim, Y. A., Park, S., Park, S. K., Hwang, I., ... Kwon, D. S. (2011). TAXEL: Initial progress toward self-morphing visio-haptic interface. In 2011 IEEE World Haptics Conference, WHC 2011 (pp. 37-42). https://doi.org/10.1109/WHC.2011.5945458 
Leithinger, D. (2010). Relief: A Scalable Actuated Shape Display. In Proceedings of the fourth international conference on Tangible, embedded, and embodied interaction (pp. 221222). https://doi.org/10.1145/1709886.1709928

Longcore, T., \& Rich, C. (2004). Ecological light pollution. Frontiers in Ecology and the Environment. https://doi.org/10.1890/1540-9295(2004)002[0191:ELP]2.0.CO;2

Maes, P. (2002). Agents that reduce work and information overload. Communications of the ACM, 37(7), 30-40. https://doi.org/10.1145/176789.176792

Mankoff, J., \& Dey, A. (2003). From conception to design: a practical guide to designing ambient displays. In Public and Situated Displays. https://doi.org/10.1055/s-20081067188

Matviienko, A., Rauschenberger, M., Cobus, V., Timmermann, J., Fortmann, J., Löcken, A., ... Boll, S. (2015). Towards New Ambient Light Systems: a Close Look at Existing Encodings of Ambient Light Systems. Interaction Design and Architecture(s) Journal, 26(Special issue on: Designing for Peripheral Interaction: seamlessly integrating interactive technology in everyday life), 10-24. Retrieved from http://www.mifav.uniroma2.it/inevent/events/idea2010/doc/26_1.pdf

Mensvoort, K. (2012). Information decoration: Our environment as an information carrier. Next Nature (1st ed.). Actar, Editors: Koert van Mensvoort, Hendrik-Jan Grievink. Retrieved from https://www.researchgate.net/publication/259980044_Information_Decoration_our_ environment_as_information_carrier

Nakatani, M., Kajimoto, H., Sekiguchi, D., Kawakami, N., \& Tachi, S. (2003). 3D Form Display with Shape Memory Alloy. Virtual Reality, 8th(1342-4564), 179-184. Retrieved from http://citeseerx.ist.psu.edu/viewdoc/download?doi=10.1.1.109.883\&amp;rep=rep1\&a mp;type $=$ pdf

Nojima, T., Ooide, Y., \& Kawaguchi, H. (2013). Hairlytop interface: An interactive surface display comprised of hair-like soft actuators. In 2013 World Haptics Conference, WHC 2013 (pp. 431-435). https://doi.org/10.1109/WHC.2013.6548447

Norman, D. A. (2002). The Design of Everyday Things. The Electronic Journal of Communication. https://doi.org/10.1002/hfm.20127

Norman, D. A. (2010). The way I see it: Natural user interfaces are not natural. Interactions, 17(3), 6. https://doi.org/10.1145/1744161.1744163

Offermans, S. (2016). Interacting with Light. PhD Dissertation. Retrieved from https://www.narcis.nl/publication/RecordID/oai:pure.tue.nl:publications\%2Fbbbfcfcc -10bb-45a7-ab20-e520b3516c23

Offermans, S. A. M., van Essen, H. A., \& Eggen, J. H. (2014). User interaction with everyday lighting systems. Personal and Ubiquitous Computing, 18(8), 2035-2055. https://doi.org/10.1007/s00779-014-0759-2 
Olwal, A., Cheng, N., Follmer, S., Ishii, H., \& Leithinger, D. (2012). Jamming user interfaces. In Proceedings of the 25th annual ACM symposium on User interface software and technology - UIST '12 (p. 519). https://doi.org/10.1145/2380116.2380181

Poupyrev, I., Nashida, T., \& Okabe, M. (2007). Actuation and tangible user interfaces. In Proceedings of the 1st International Conference on Tangible and Embedded Interaction (p. 205). https://doi.org/10.1145/1226969.1227012

Pousman, Z., \& Stasko, J. (2006). A taxonomy of ambient information systems. Proceedings of the International Working Conference on Advanced Visual Interfaces (AVI '06), 67. https://doi.org/10.1145/1133265.1133277

Prante, T., Röcker, C., Streitz, N., Stenzel, R., Magerkurth, C., van Alphen, D., \& Plewe, D. (2003). Hello.Wall - Beyond Ambient Displays. In Adjunct Proceedings of the International Conference on Ubiquitous Computing (UbiComp'03) (pp. 277-278). https://doi.org/10.1.1.58.3459

Rasmussen, M. K. ., Pedersen, E. W. ., Petersen, M. G. ., \& Hornbæk, K. . (2012). Shapechanging interfaces: A review of the design space and open research questions. Conference on Human Factors in Computing Systems - Proceedings. https://doi.org/10.1145/2207676.2207781

Review, D. K.-H. S., \& 1988, undefined. (1988). Time and space in the work of László MoholyNagy. Epa.Niif.Hu, $\quad 15 . \quad$ Retrieved from http://epa.niif.hu/00000/00010/00018/pdf/HSR_1988_1_063-076.pdf

Scupin, R. (2015). The KJ Method: A Technique for Analyzing Data Derived from Japanese Ethnology. Human Organization, 56(2), 233-237. https://doi.org/10.17730/humo.56.2.x335923511444655

Taher, F., Hardy, J., Weichel, C., Jansen, Y., Hornbæk, K., Karnik, A., \& Alexander, J. (2015). Exploring Interactions with Physically Dynamic Bar Charts. In Proceedings of the 33rd Annual ACM Conference on Human Factors in Computing Systems (pp. 3237-3246). https://doi.org/10.1145/2702123.2702604

Taher, F., Vidler, J., \& Alexander, J. (2017). A Characterization of Actuation Techniques for Generating Movement in Shape-Changing Interfaces. International Journal of Human-

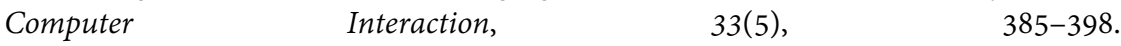
https://doi.org/10.1080/10447318.2016.1250372

Tang, S. K., Sekikawa, Y., Leithinger, D., Follmer, S., \& Ishii, H. (2014). Tangible CityScape. Retrieved March 9, 2019, from http://tangible.media.mit.edu/project/tangiblecityscape

Tomitsch, M., Grechenig, T., Vande Moere, A., \& Renan, S. (2008). Information sky: Exploring the visualization of information on architectural ceilings. In Proceedings of the International Conference on Information Visualisation (pp. 100-105). https://doi.org/10.1109/IV.2008.81

Vertegaal, R. (2007). Organic User Interfaces (Oui!): Designing Computers in Any Way Shape 
or Form. In Unconventional Computation (pp. 28-28). https://doi.org/10.1007/978-3540-73554-0_4

Vogel, D., \& Balakrishnan, R. (2005). Interactive public ambient displays. In Proceedings of the International Symposium on User Interface Software and Technology (UIST'04) (p. 137). https://doi.org/10.1145/1029632.1029656

Wagner, P., Malisz, Z., \& Kopp, S. (2014). Gesture and speech in interaction: An overview. Speech Communication, 57, 209-232. https://doi.org/10.1016/j.specom.2013.09.008

Weiser, M. (2007). The computer for the 21 st century . ACM SIGMOBILE Mobile Computing and Communications Review, 3(3), 3-11. https://doi.org/10.1145/329124.329126

Weiser, M., \& Brown, J. S. (1995). Designing Calm Technology. World Wide Web Internet And Web Information Systems. https://doi.org/10.1.1.135.9788

Wu, J., Van Essen, H., \& Eggen, B. (2016). Designing sculpting light systems for information decoration. In TEI 2016 - Proceedings of the 10th Anniversary Conference on Tangible Embedded and Embodied Interaction. https://doi.org/10.1145/2839462.2856547

Yao, L., Niiyama, R., Ou, J., Follmer, S., Della Silva, C., \& Ishii, H. (2013). PneUI: Pneumatically Actuated Soft Composite Material s for Shape Changing Interfaces. In Proceedings of the 26th annual ACM symposium on User interface software and Technology (pp. 13-22). https://doi.org/10.1145/2501988.2502037

Yao, L., Ou, J., Cheng, C., Steiner, H., Wang, W., Wang, G., \& Ishii, H. (2015). bioLogic : Natto Cells as Nano actuators for Shape Changing Interfaces. In In Proceedings of the 33rd Annual ACM Conference on Human Factors in Computing Systems (CHI '15). https://doi.org/10.1145/2702123.2702611

Yarin, P., Ishii, H., Gorbet, M., Dahley, A., Ullmer, B., Wisneski, C., \& Brave, S. (2007). Ambient Displays: Turning Architectural Space into an Interface between People and Digital Information. In International Workshop on Cooperative Buildings (pp. 22-32). https://doi.org/10.1007/3-540-69706-3_4

Zimmerman, J., Forlizzi, J., \& Evenson, S. (2007). Research through design as a method for interaction design research in HCI. In Proceedings of the SIGCHI conference on Human factors in computing systems (p. 493). https://doi.org/10.1145/1240624.1240704 

Appendices 


\section{Appendix I: The 33 state-of-the-art examples of SLS}

In this appendix, we present the sample of 33 related examples of SLS (published between 1998 and 2016) that are used in the analysis of generating the SLS building blocks. They meet both criteria: the system was designed by combining shape change with light; and the shape change of physical objects influences the light path and results in manipulable light patterns. For each example, the following information is presented: title, a characteristic picture, the authors or creators, the publication time, links to the original sources, a link to a video demonstrating the object if available, and a short description.

\section{No. 01. All Lamp}

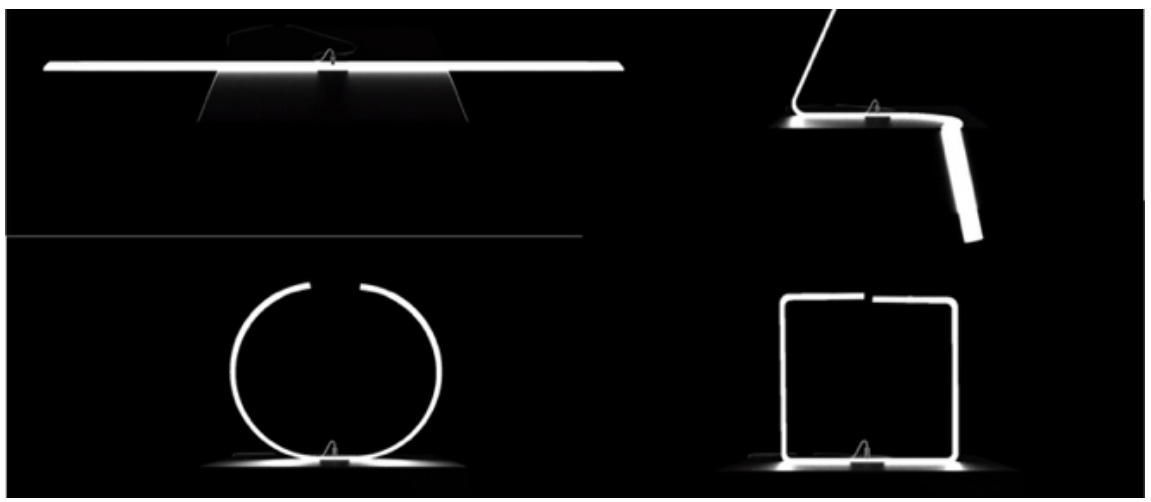

Tobias Lugmeier, 2014

Retrieved from: http://tobiaslugmeier.com/work/alllamp/

ALL LAMP is strip of deformable lamp. It is a decorative object during daytime, but a "unnatural object" when it is plugged in. By its flexibility, it can be put and even hung up at everywhere.

No. 02. Babele
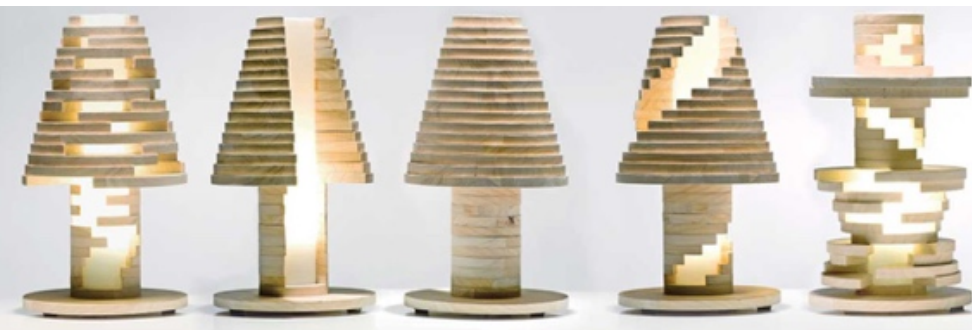
Manifattura Italiana Design M.I.D. Srl, 2013

Retrieved from: http://www.designmid.it/eng/babele.php

Babele enables people to create different forms of light gaps by assembling its modularized wood shades.

\section{No. 03. Breathing Wall}

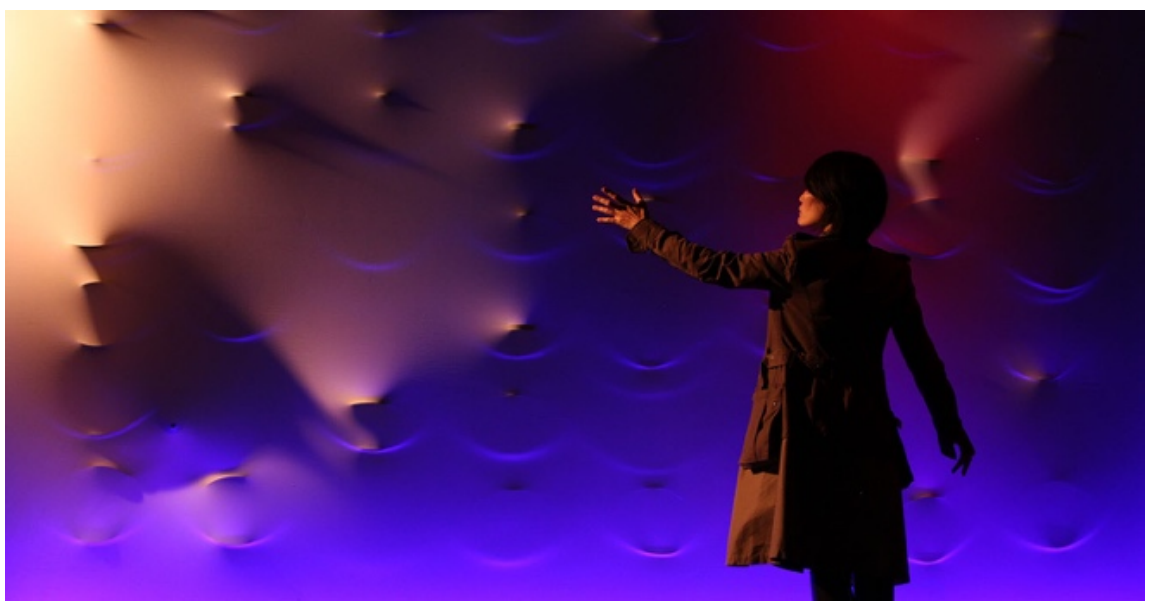

Behnaz Farahi, 2014

Retrieved from: http://www.behnazfarahi.com/the-living-breathing-wall/

Video source: https://vimeo.com/81572631

Breathing Wall is an interactive art installation, which responses people's gestures in front of it by changing its fabric's textures.

\section{No. 04. Drones Light Show}

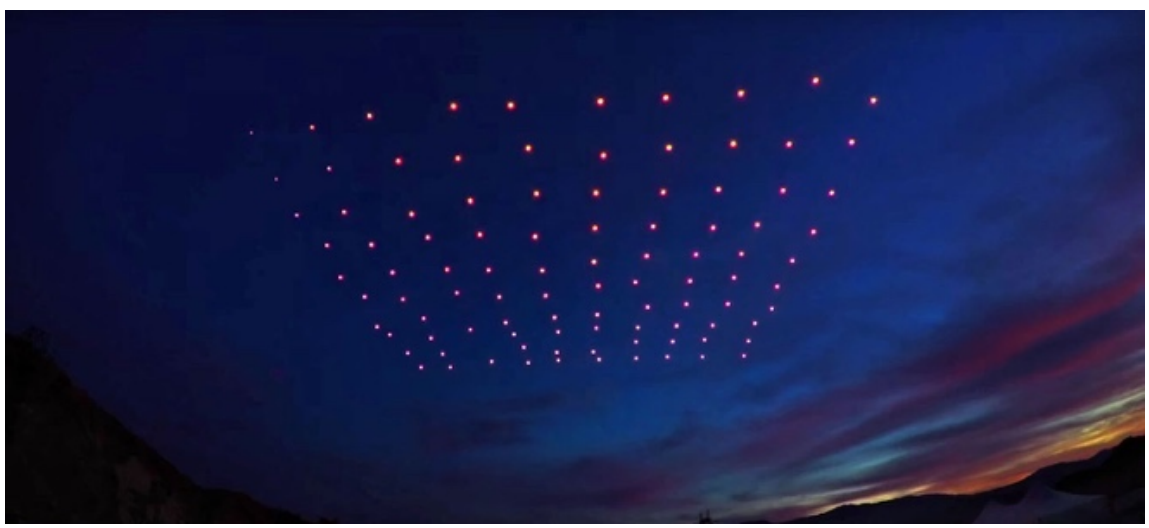


Intel, 2015

Retrieved from: https://www.youtube.com/watch?v=aOd4-T_p5fA

Drones Light Show employs hundreds of Intel drones to brighten up the night sky with a choreographed light performance in 3 dimensions.

No. 05. Electric Sky

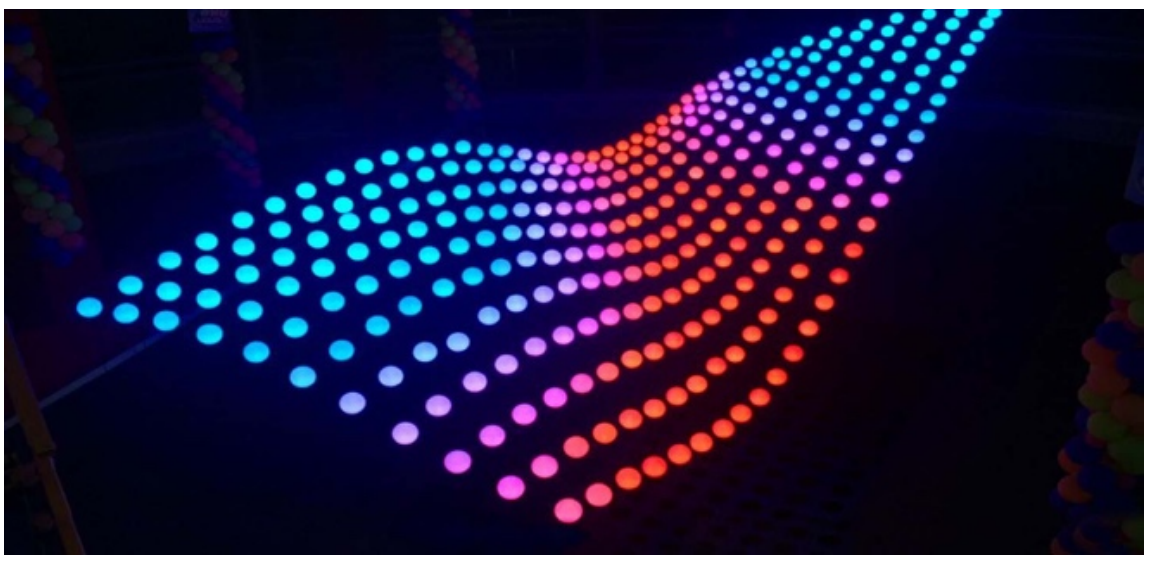

KINETIC LIGHTS, 2016

Retrieved from: https://www.kinetic-lights.com/portfolio/electric-sky-2

Video source: https://vimeo.com/233184440

KINETIC LIGHTS is a kinetic lighting installation, which is consist of 320 kinetic RGB light sources. It has been exhibited in many unique venues with restaurant, cocktail bar, lounge and private karaoke pods.

\section{No. 06. Eternal Sunset}

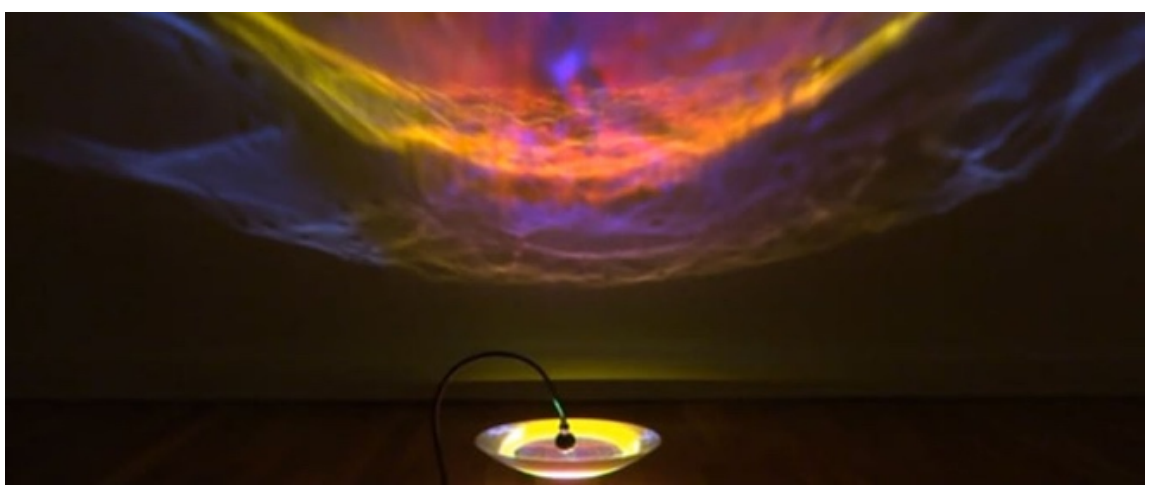


Laurent Fort, 2014

Retrieved from: https://www.customartlight.com/eternal-sunset

Video source: https://vimeo.com/191648808

Eternal Sunset creates fantastic and ever-changing projected light patterns, which are reflected from a Dichroic filter floating and turning in the water.

\section{No. 07. Flow}

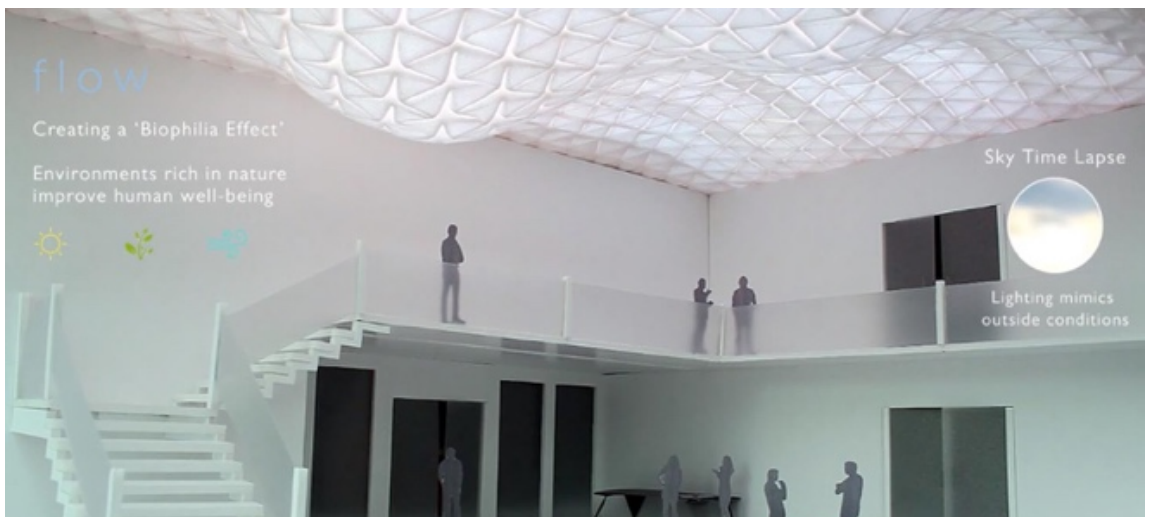

Chris Selway, 2015

Video source: $\underline{\text { https://vimeo.com/144424152 }}$

Flow is a conceptual lighting project which demonstrates the possibilities of designing kinetic lighting system to improve user well-being within interior environment.

\section{No. 08. Homeostatic Facade System}

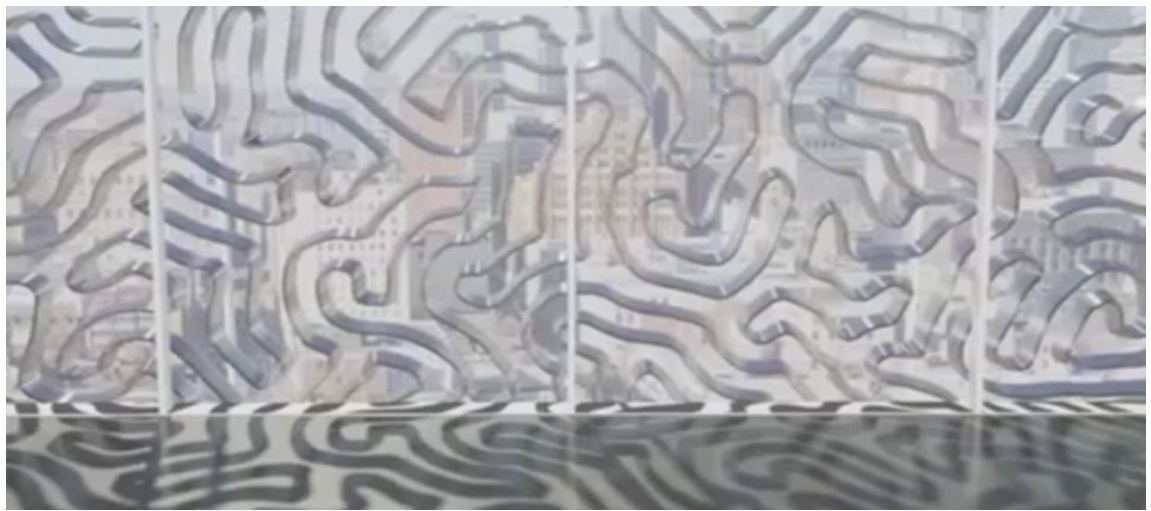


Decker Yeadon, 2011

Retrieved from: https://materialdistrict.com/article/homeostatic-facade-system/

Video source: https://www.youtube.com/watch?v=U2tSZ89bwPY

Homeostatic Façade System is a self-regulating façade system that automatically adjusts the amount of light that gets into the exterior environments.

\section{No. 09. Kangaroo Light}

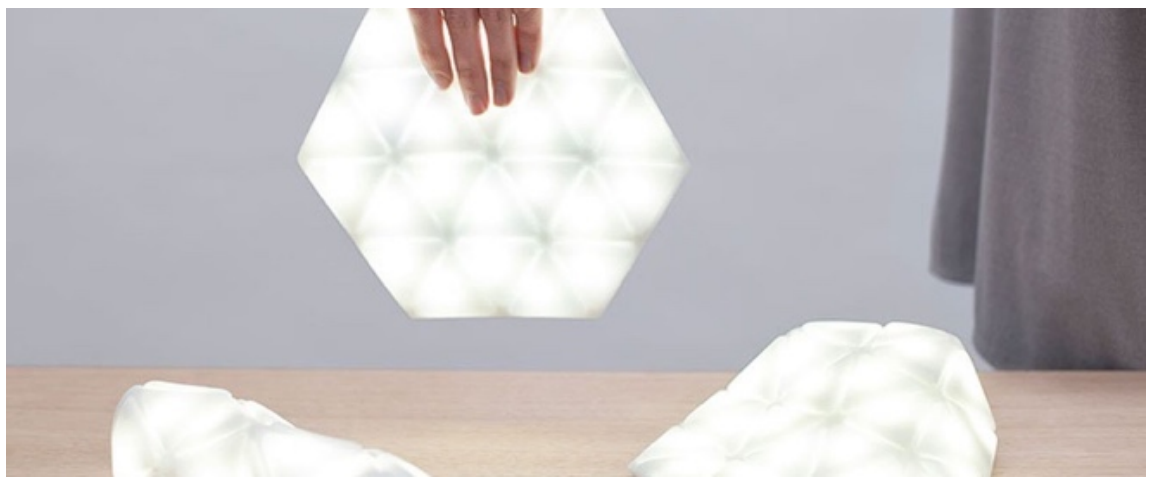

Studio Banana, 2014

Retrieved from: https://www.kickstarter.com/projects/studio-banana/kangaroo-light Video source: https://vimeo.com/95299083

KANGAROO LIGHT is a portable and flexible light. By putting it into the bags, it provides a soft glow when people are locating things without frustration.

\section{No. 10. KIHOU}

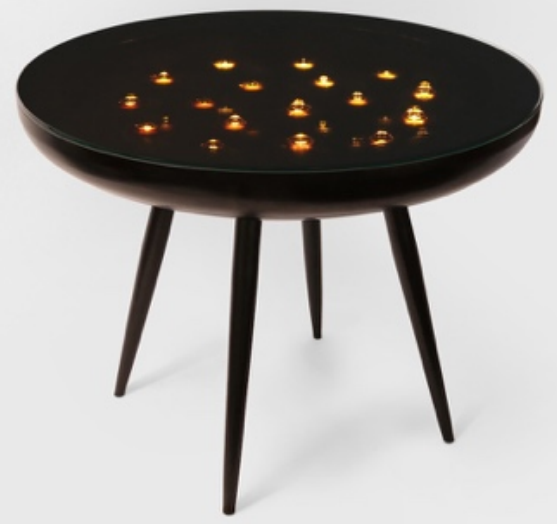


Tangent, 2015

Retrieved from: http://www.tangent.uk.com/collection/kihou-table/

Video source: $\underline{\text { https://vimeo.com/120691446 }}$

Kihou is a lighting product which presents breathing-like illuminated air bubbles rhythmically on the surface of a black table.

\section{No. 11. KINETIC Wall of Light}

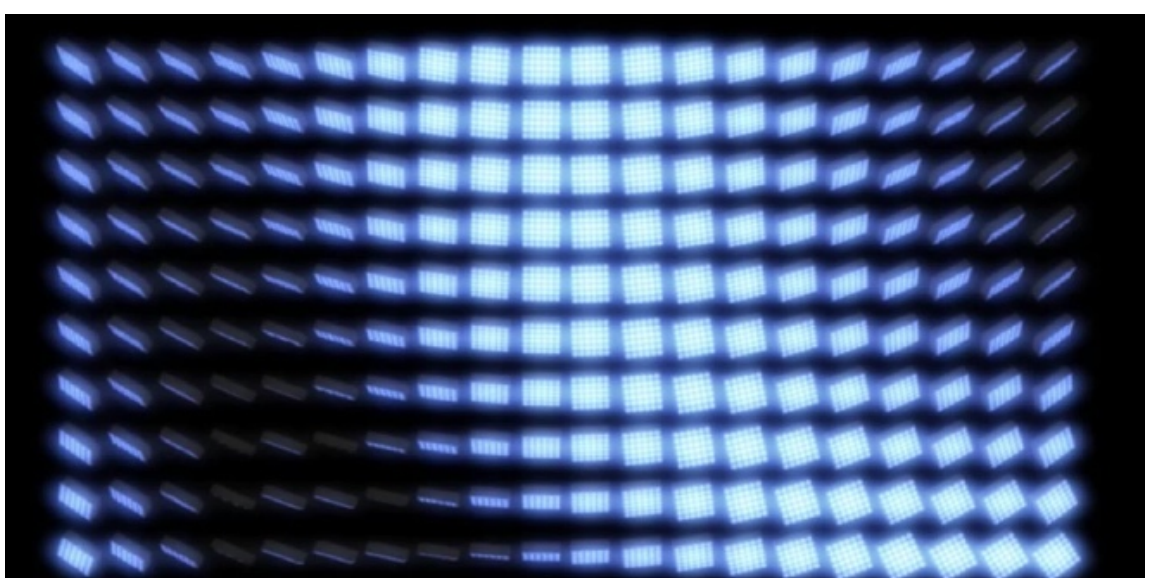

Pixelux Studio, 2014

Retrieved from: https://www.pixelux.tv/kinetic-wall-of-light

Video source: https://vimeo.com/107353028

KINETIC Wall of light is composed by a grid of $20 \times 10$ moving light panels. It reveals graphics that are generated by the motion of the fixtures to create visual experiences of optical illusion and light distortion.

\section{No. 12. Lotus}

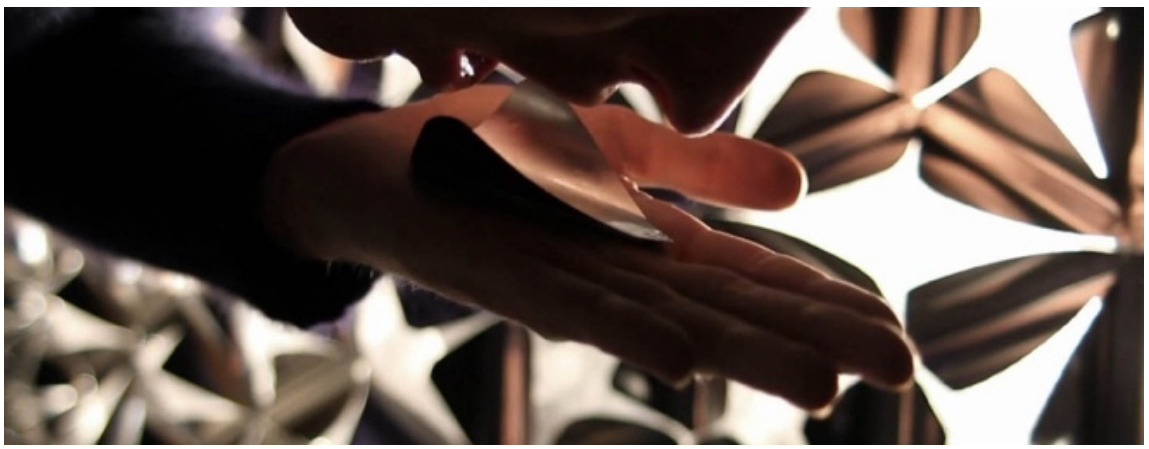


Studio Roosegaarde, 2010

Retrieved from: https://studioroosegaarde.net/project/lotus

Video source: https://vimeo.com/51065809

Lotus is an interactive light installation, which employs hundreds of ultra-light aluminium flowers to produce responsible light gaps. When people are approaching to it, the big silver dome lights up and opens its flowers.

\section{No. 13. Lucy Light Forest}

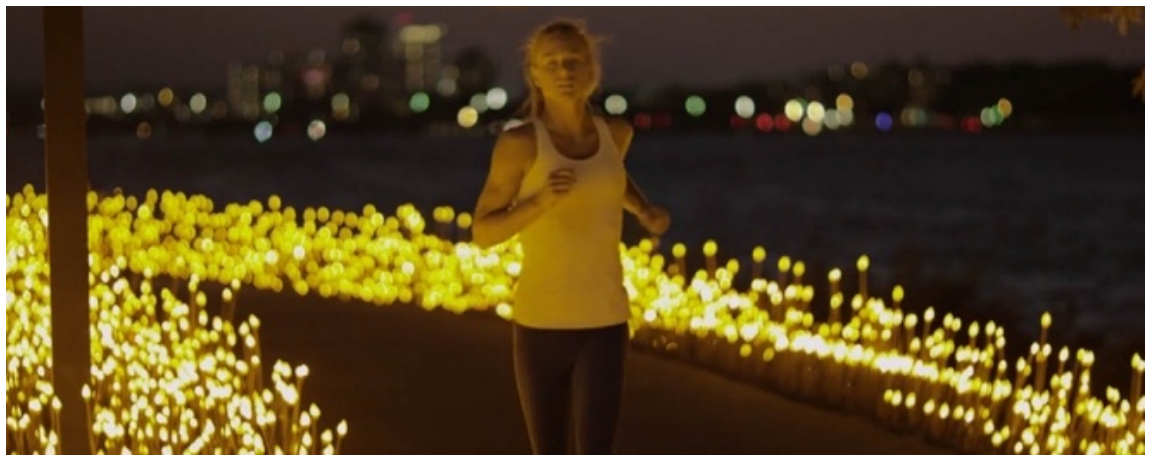

Dawn Dzedzy, 2013

Retrieved from: https://www.dawndzedzy.com/lucy-light-forest

Video source: https://vimeo.com/207381612

Lucy Light Forest is an interactive light installation, which consists of more than 10,000 solar-powered LED lights. It can respond to the people's movement by detecting the sounds surround it.

\section{No. 14. Lumio}

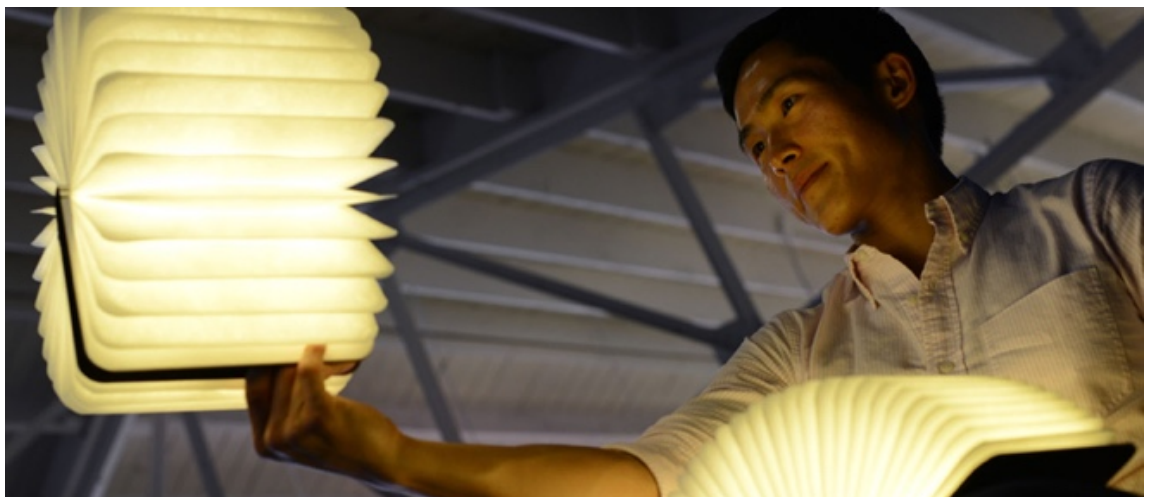


Max Gunwan, 2013

Retrieved from: https://www.hellolumio.com

Video source: https://vimeo.com/112128027

Lumio is an interactive lamp, which is formed as a hardcover book. When the "book" is opened, the light is turned on accordingly.

\section{No. 15. Lungplant}

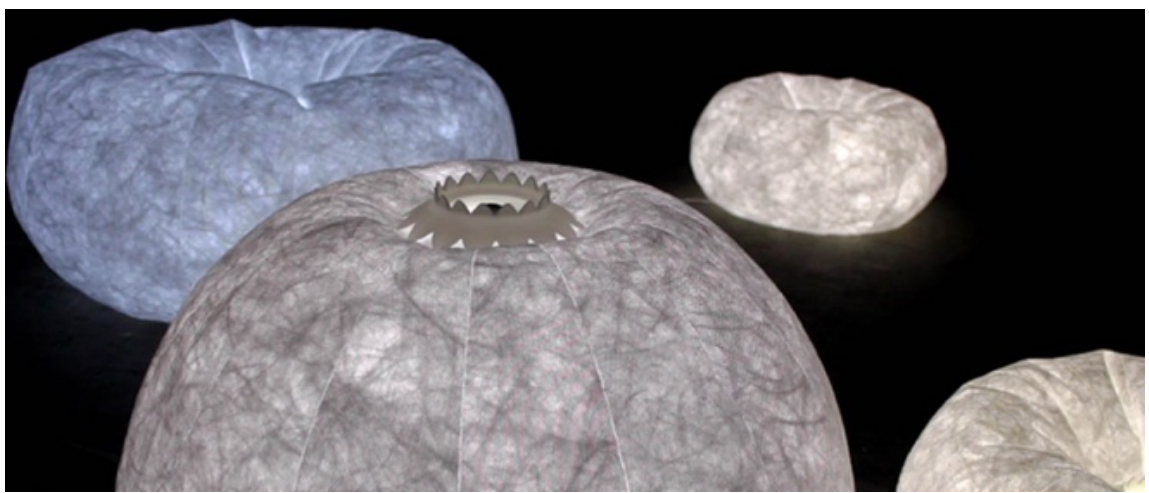

Tim van Cromvoirt, 2013

Retrieved from: http://timvancromvoirt.com/portfolio/lungplant

Video source: https://vimeo.com/61519776

Lungplant is a kinetic light installation, which simulates the human's breathing patterns by inflating/deflating its lamp shades and brightening/dimming its inner light sources.

No. 16. Manta Rhei

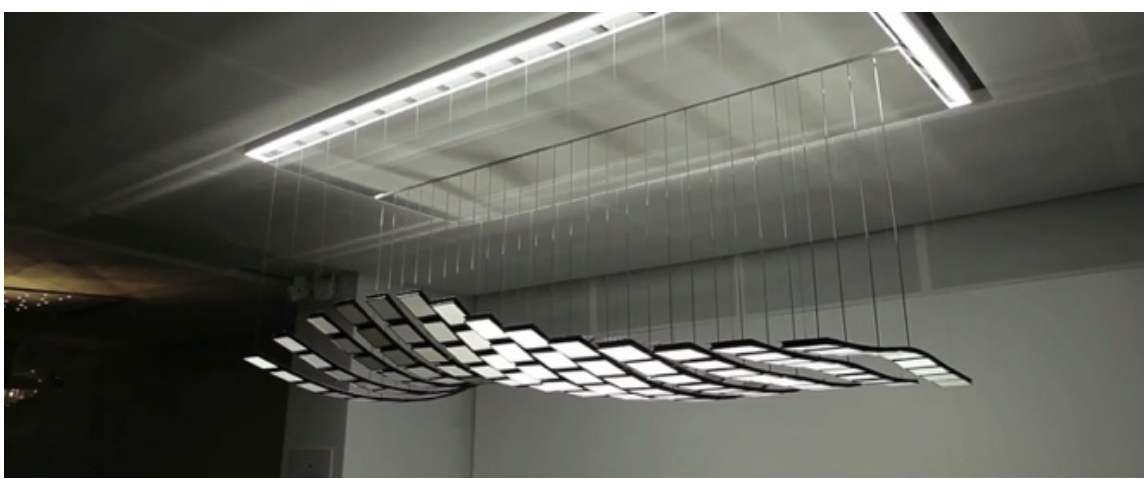

ART+COM Studios, 2012 
Retrieved from: https://artcom.de/en/project/manta-rhei/

Video source: https://vimeo.com/46289124

Manta Rhei is a luminous kinetic sculpture, which combines choreographed light with physical movement. It can perform a wide range of patterns to show the impression that is either harmonious or conflicting.

\section{No. 17. MegaFaces Pvilion}

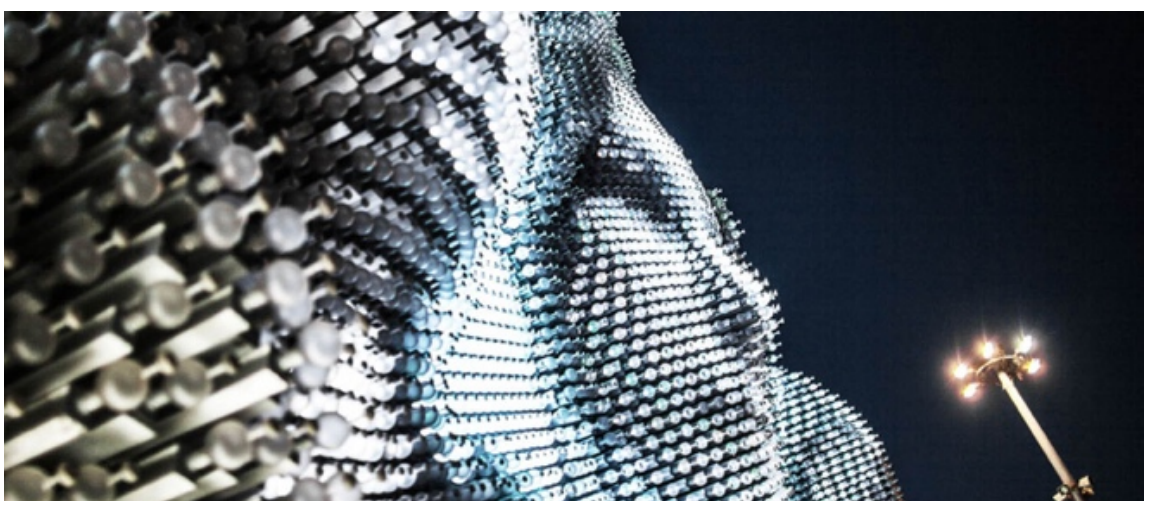

Asif Khan Ltd., 2014

Retrieved from: http://www.asif-khan.com/project/sochi-winter-olympics-2014/

Video source: https://vimeo.com/99030884

MegaFaces Pavilion is a kinetic light sculpture exhibited at the Sochi 2014 Winter Olympics. It presents a new form of sculpture which is glowing and dynamic rather than cold and static.

\section{No. 18. MOBILITY}

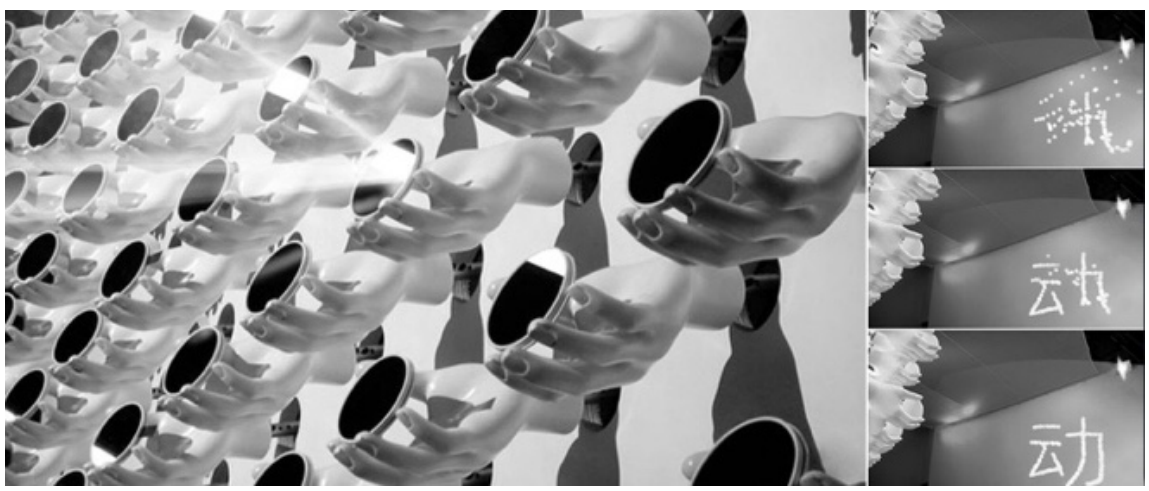

ART+COM Studios, 2010

Retrieved from: https://artcom.de/en/project/mobility/ 
Video source: https://vimeo.com/67559505

Mobility is a kinetic light installation, in which a bright light source shines on a matrix of turning mirrors to cast small moving light spots on a white wall. These light spots are used to form a Chinese character, which means motion.

No. 19. NEBULA

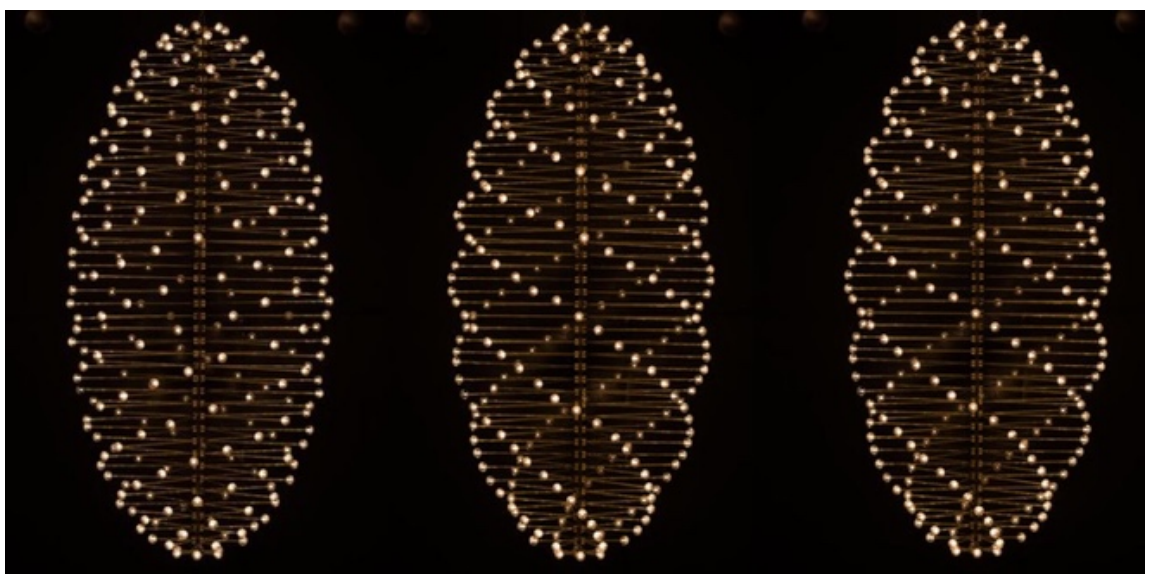

INK Design Studio, 2014

Retrieved from: http://www.inklighting.com/nebulachandelier Video source: https://vimeo.com/169616005

NEBULA is a kinetic light installation that enables people to create their own light sculptures by controlling the luminosity, spinning speed, and spinning direction of its light fixtures.

\section{No. 20. Origami Snow}

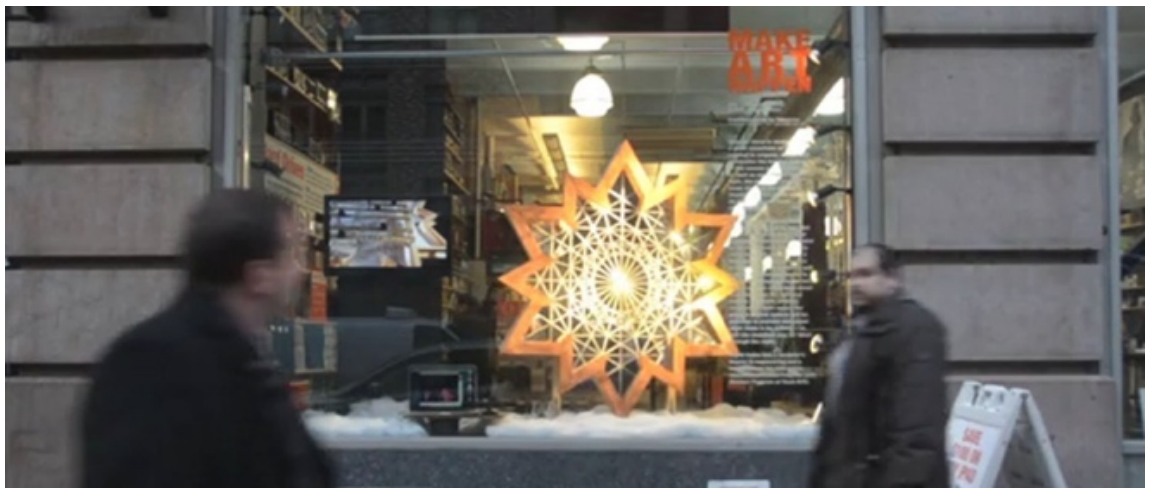


Ingrid Gabor, 2013

Retrieved from: http://ingridgabor.com/work/light-waves-interactive-sculpture/ Video source: https://vimeo.com/82379175

Origami Snow is an interactive sculpture that reacts to the presence of people passing by. It's composed of laser-cut mirrored pieces that are motorized from behind to add subtle movement to the whole piece, enough to scatter reflections of light that create a shimmery effect on the sidewalk.

\section{No. 21. Parametric Space}

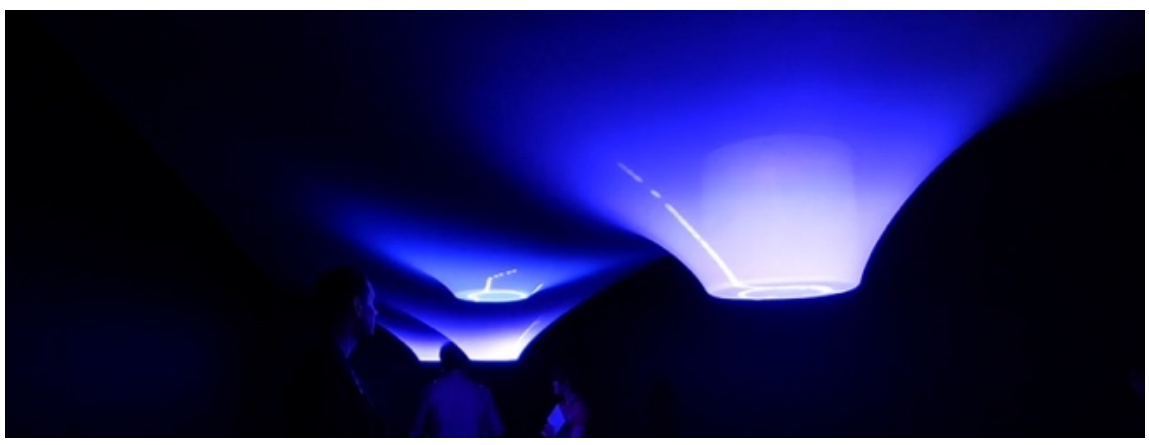

Zaha Hadid \& Kollision, 2013

Retrieved from: $\underline{\text { http://kollision.dk/en/parametric }}$

Video source: https://vimeo.com/69356542

Parametric Space presents an interactive installation, which reacts to the visitors' movements by changing shape and light projections.

\section{No. 22. KINETICS}

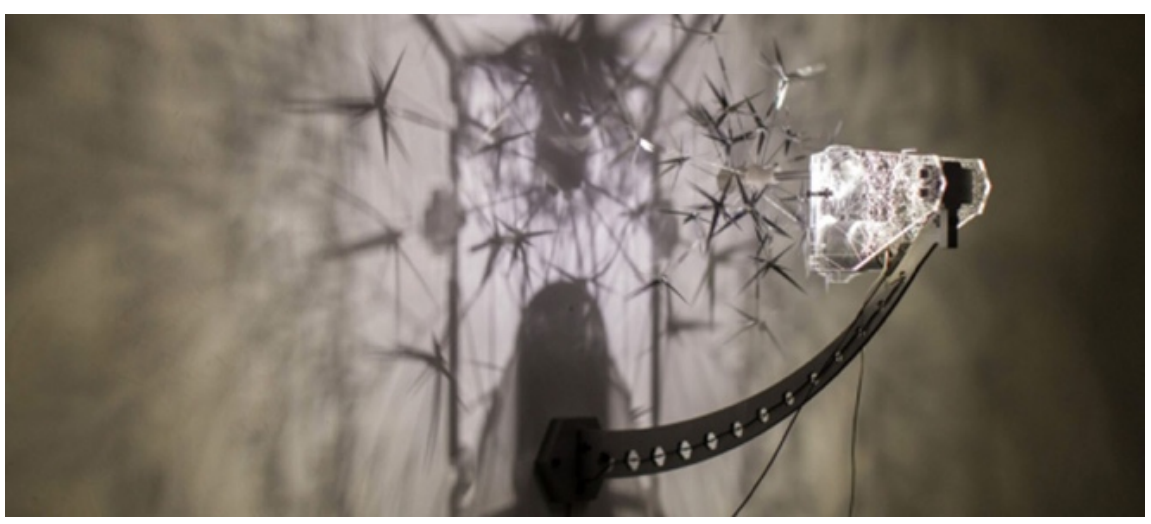


Paul Myoda, 2014.

Retrieved from: http://www.paulmyoda.com/index.php?/-kinetics/.

Video source: https://vimeo.com/82157700

KINETICS presents a dynamic light patterns and shadows on the walls by vibrating its mechanized sculpture.

No. 23. Resonance

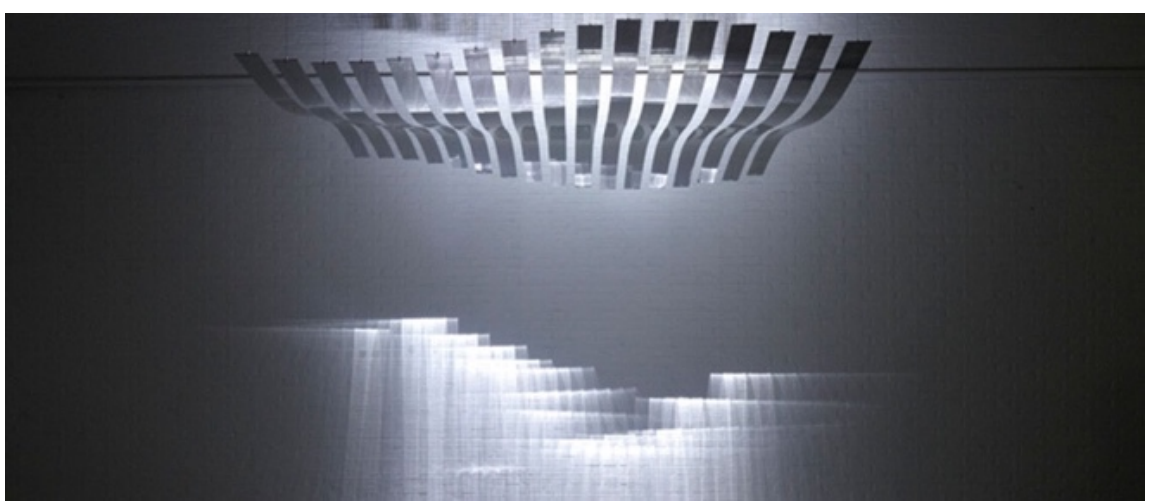

ART+COM Studios, 2010

Retrieved from: https://artcom.de/en/project/resonance/

Video source: https://vimeo.com/67561030

Resonance is a kinetic light installation, in which an array of metal blades changes their orientations to create dynamic wave-like light projections on the wall.

No. 24. Shade

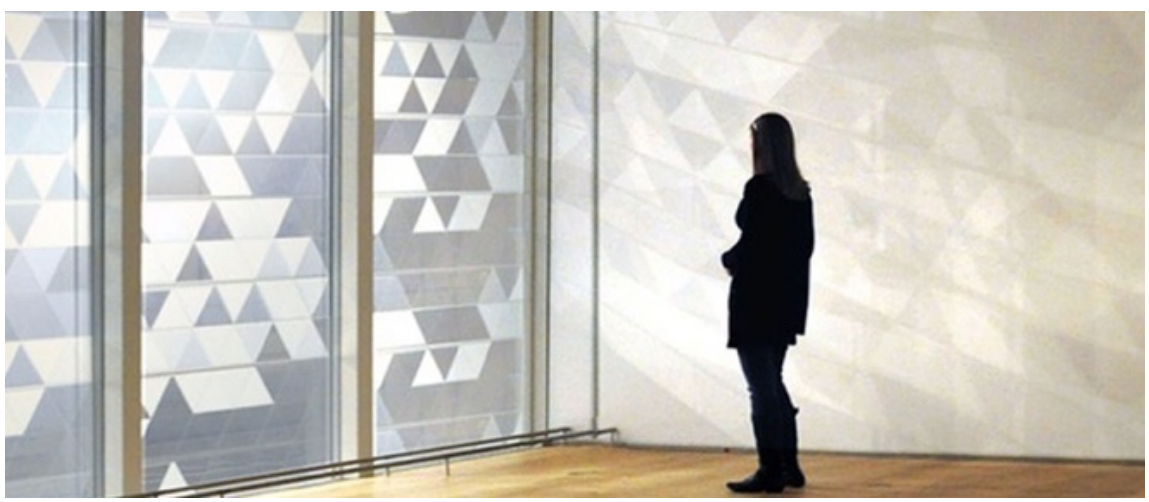


Simon Heijdens, 2010

Retrieved from: http://www.simonheijdens.com/indexbig.php?type=project\&name=Shade Video source: https://vimeo.com/106669679

Shade employs a unique type of glasses, which transparency can be changed, to augment the perception of sunlight indoor. When wind moves over the glasses, a living pattern of light appears dynamically on the windows and the wall.

\section{No. 25. Slash Lamp}

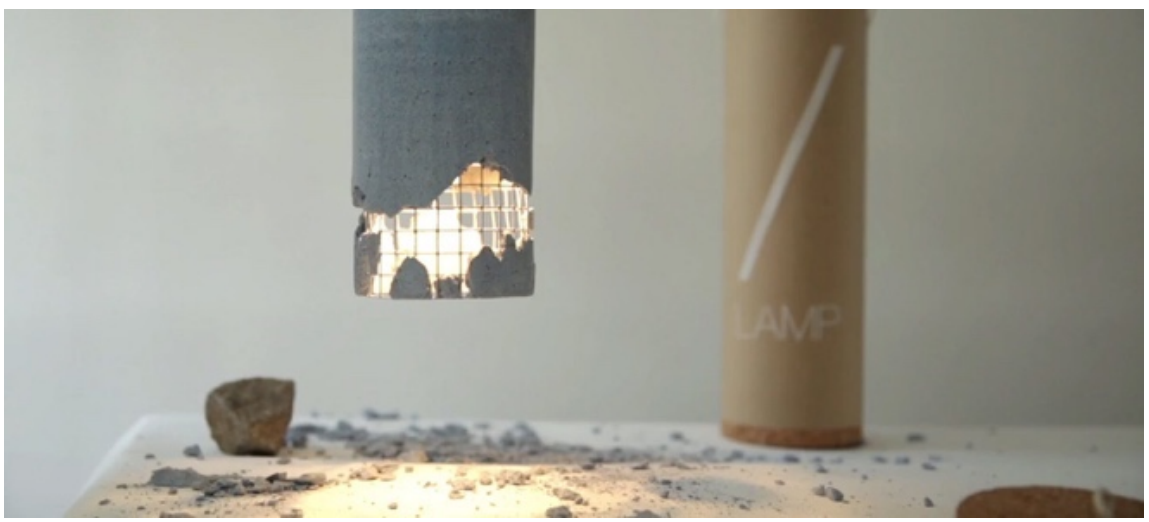

Dragos Motica, 2014

Retrieved from: http://www.dragosmotica.ro/portfolio/lamp/ Video source: https://vimeo.com/100428174

Slash Lamp enables the user to create his/her own lampshade by smashing its concrete cover.

No. 26. Soak

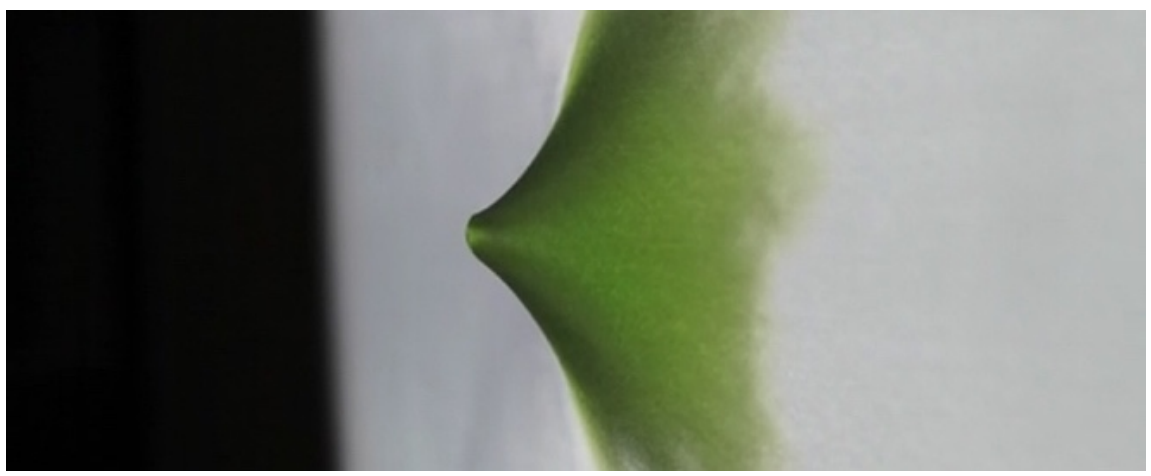

Everyware Group, 2011 
Retrieved from: http://everyware.kr/home/portfolio/soak/

Video source: https://vimeo.com/27158491

Soak is an interactive installation, which reacts the user that is touching on a fabric with animated projections.

No. 27. Soft Light

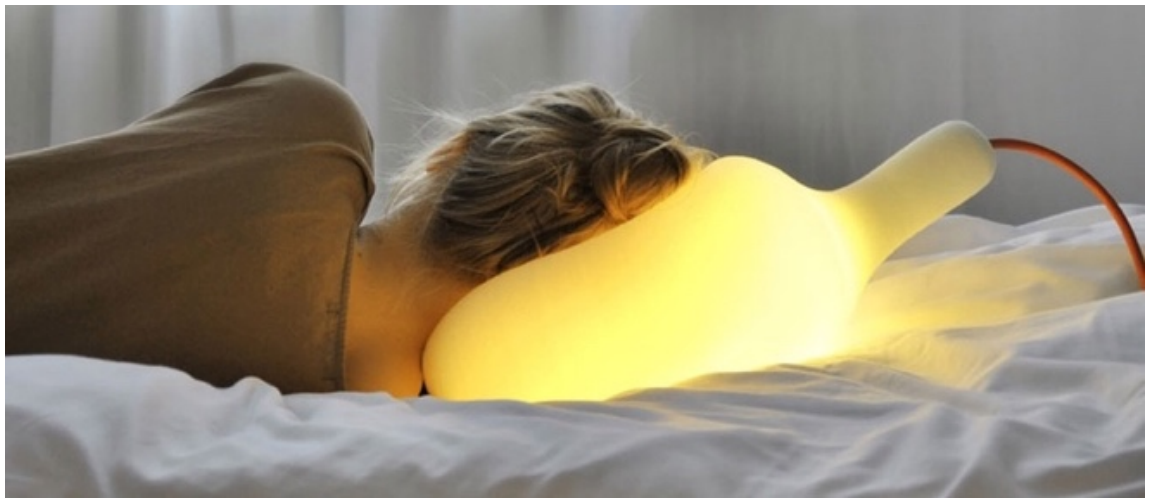

Simon Frambach, 2014

Retrieved from: http://www.simon-frambach.com/simon-frambach.html\#work

Soft Light is a flexible light shade made of foamed polyurethane.

\section{No. 28. Digital Sundial}

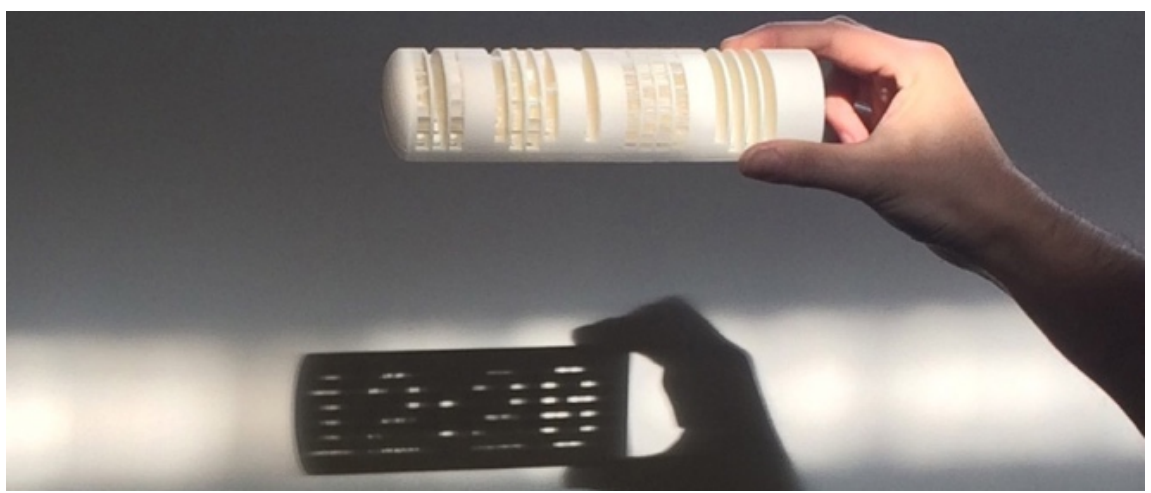

Julldozer, 2015

Retrieved from: http://www.mojoptix.com/2015/10/25/mojoptix-001-digital-sundial/

Video source: https://www.youtube.com/watch?v=wrsje5It UU 
Digital Sundial produces the shadows of digital colour by blocking the sunlight with a 3D-printed "magic tube."

\section{No. 29. Spine}

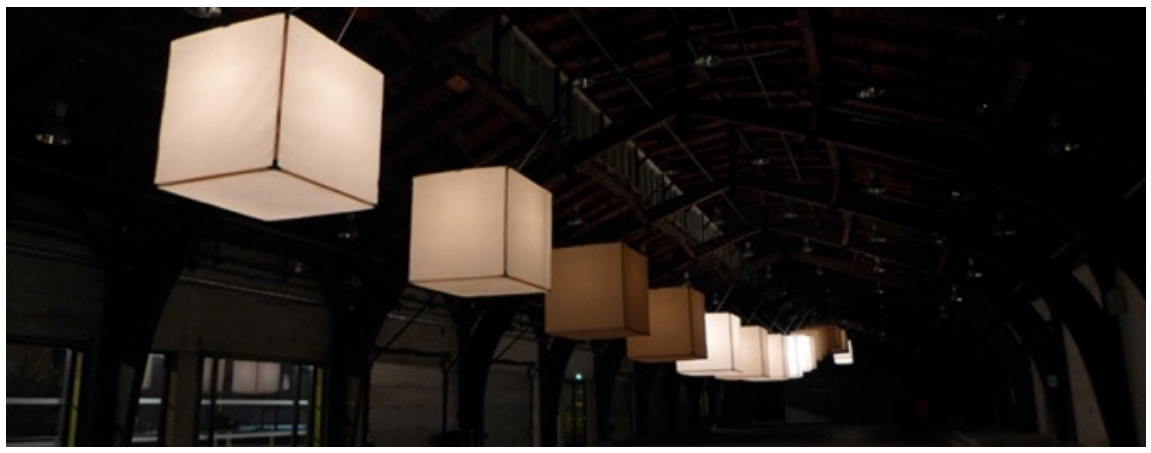

Kollision, 2012

Retrieved from: http://kollision.dk/en/spine

Video source: https://vimeo.com/55596743

Spine is a kinetic light installation, in which twenty glowing cubes move up and down to produce fluid motions and flowing light.

\section{No. 30. String Waves}

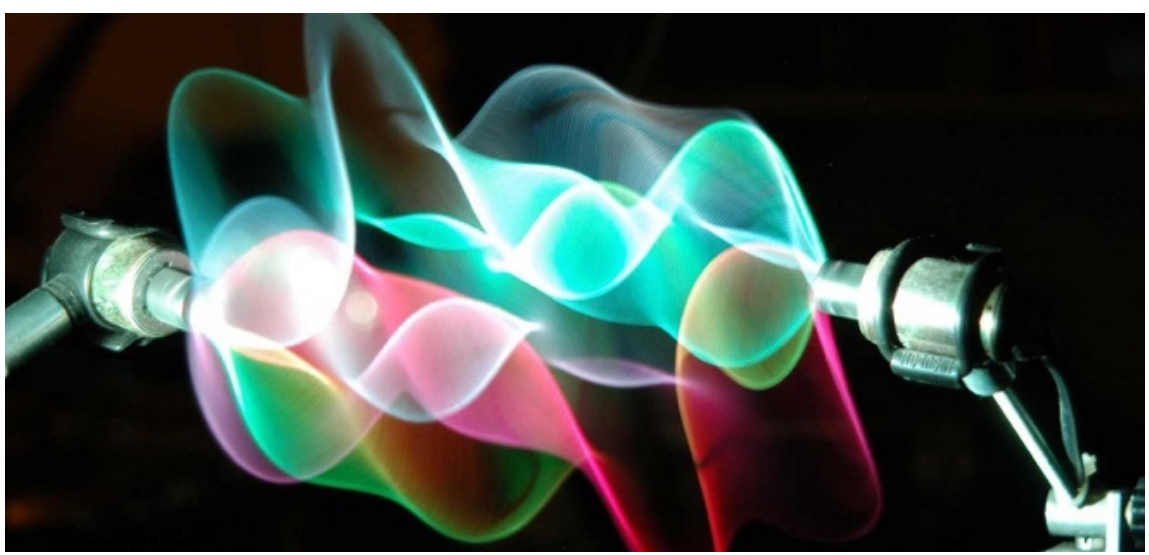

Paul Friedlander, 2015

Retrieved from: http://www.paulfriedlander.com/Light Sculpture.html

Video source: https://vimeo.com/100402097 
String Waves is a kinetic light sculpture, in which the rope is rotated quickly to present the reflected light by different dynamic forms.

No. 31. Tangeez

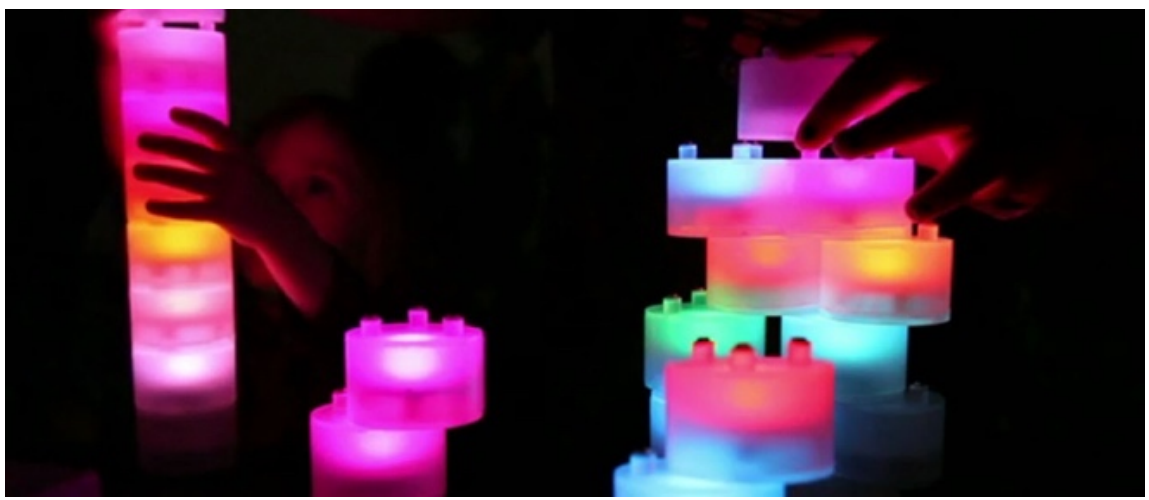

Tangible Lights, LLC., 2013

Retrieved from: http://www.tangeez.us

Video source: https://vimeo.com/59975769

Tangeez is a set of interactive toys, which provides kids with building modules that light-up and change colour when they play with them.

\section{No. 32. The Plus Pendant}

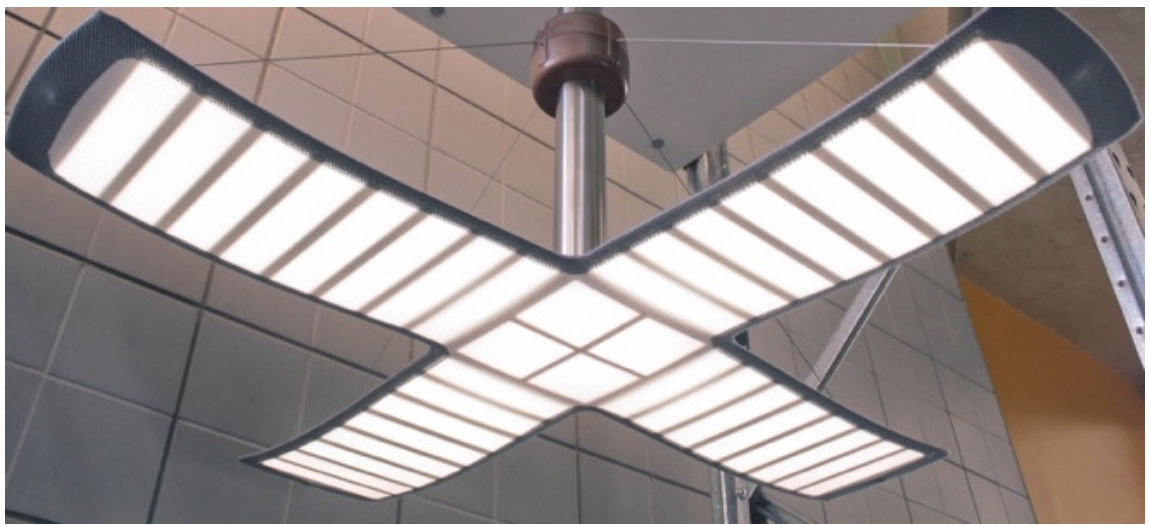

Andy Zhou, 2013

Retrieved from: https://www.youtube.com/watch?v=7RfKImXo200 
The Plus Pendant is a kinetic OLED luminaire designed to showcase the flexibility of the organic light emitting diode technology.

\section{No. 33. Tri}

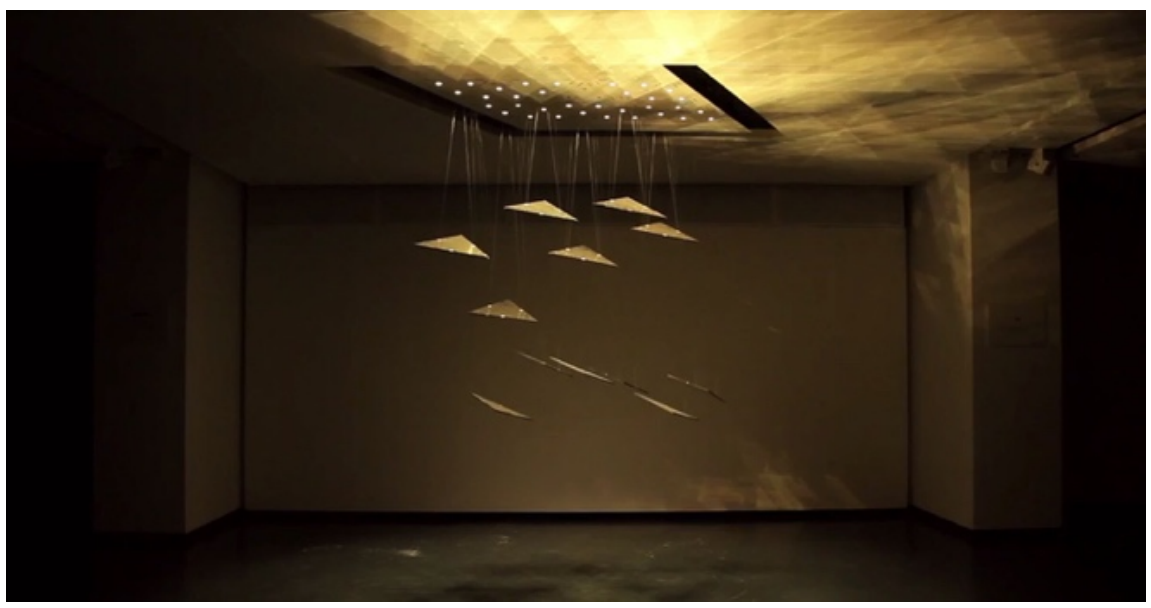

ART+COM Studios, 2013

Retrieved from: https://artcom.de/project/symphonie-cinetique/ Video source: $\underline{\text { https://vimeo.com/81100379 }}$

Tri is a kinetic installation, in which triangular mirrors move in a three-dimensional space to produce dynamic ever-changing reflected light projections on the ceiling. 


\section{Appendix II: The detailed clues, ideas, and concepts}

In Appendix II, we will explain the clues, ideas, and concepts those are mentioned in Chapter 6 by three tables. The yellow table presents the details of 12 clues that are collected in our hypothetical example. The blue table specifies 17 ideas that generated in our hypothetical example. The green table elaborates one of the complete concepts that are developed in our hypothetical. The other complete concepts are not presented in this decertation.

\section{Clues}

\section{Raw Datas}

CLUE 1: Buienalarm can be a reliable source of raw data. Because it is widely used in the target user group.

Processing and Processed Data

CLUE 2: Focusing on the rain condition in 30 mins. Because that is what users concern most, according to the description in the given design brief (see section 6.2).

CLUE 3: Focusing on the local rain condition. Because that is what users concern most, according to the description in the given design brief (see section 6.2).

CLUE 4: Sorting the raw data into four clusters which represent four levels of rain conditions. Because that is how the users understand the rain forecast (heavy, moderate, light, and no rain) by using Buienalarm.

Mapping and Abstract Representation

CLUE 5: The transitions between different mappings of raining conditions should be smooth. For example, transition from the mapping of light rain to the mapping of moderate rain should be smooth. This is considering the aesthetic quality and subtleness of the information displays.

CLUE 6: The blue colour can represent rain conditions. This is the natural association between rain and colour. Also, the blue colour is a key element in the data visualization of Buienalarm.

Combining and Specified Presentation 
CLUE 7: The way of producing light patterns can refer to how people perceive the rain in nature. For example, reflections on water, refractions on window, etc. By doing so, we intend to enable the users to understand the information displays in a more instinctive way. CLUE 8: The specified presentation should be easily associated with the impression of rain. Because the target users could come from different cultures and education backgrounds.

Rendering and Physical View

CLUE 9: The light projections on the walls could be weak. Because the walls in the basement are too dark and uneven.

CLUE 10: The surroundings of the fixed route (see orange arrow in Figure 6.2) are possibly parts of the physical view. For examples, ceiling lights, black wall, table, carpet, and stairs. Therefore, the users can easily perceive the information displays.

Interaction Loops from the user side

CLUE 11: Enabling the user to check the rain conditions based on his/her needs. This provide playful interaction for the user to explore the light effect. After all, the basement looks boring.

Interaction Loops from the environment side

CLUE 12: Turning off the information displays when the basement is quiet. This can save the energy consumption.

\section{Ideas}

Abstract Representation

IDEA 1: Applying the Objects-driven SLS to represent the rain information in a subtle way without manipulating the properties of light. In this case, the Passive Lights could be the existing ceiling lights in the basement.

IDEA 2: Applying the Active SLS to represent the rain information in a vivid way. Especially when there is a heavy rain, the multi-modality displays of light and shape can be aesthetically pleasing.

IDEA 3: Applying the Lights-driven SLS to enable the user to check the rain conditions in detail by manually changing the shape of Passive Objects in a tangible way.

IDEA 4: Referring to the colour mapping of different rain conditions in Buienalarm.

IDEA 5: Changing the intensity of blue light to represent different levels of rain conditions. 
IDEA 6: Manipulating the permeability in shape to represent different levels of rain conditions. This was inspired by how we perceive the rain conditions by observing the rain drops on the water in nature.

Specified Presentation

IDEA 7: Simulating the blinking light effects of rain drops on the water in nature.

IDEA 8: Opening/closing a luminous umbrella to show the changes of rain condition.

IDEA 9: Applying the light patterns shown in Press Light (see Figure 6.8) to show the rain information in different fidelity levels.

IDEA 10: The stripe patterns (see Figure 6.2) on the carpet can be used as scales that indicate different modes of information displays.

IDEA 11: The light patterns shown in the example of Light Waves (see Figure 6.5 (right)) could be used to present the levels of rain conditions by different frequencies of vibration.

Physical View

IDEA 12: The existing ceiling lamps could be used as Passive Lights.

IDEA 13: Utilizing the spot lights on the ceiling as Active Lights to project imaged light patterns. To do so, the original light sources of these spot lights will be changed with smart light sources that can be manipulated with computer.

IDEA 14: The carpet in the basement could be used as Preset Objects to show the projected light patterns. Especially, the stripe patterns on it can be used to support different modes of information representation.

Interaction loops

IDEA 15: Adding an interaction for Mapping from the environment side. In this interaction, the representation fidelity of SLS will be altered according to the sound level in the basement.

IDEA 16: Adding an interaction for Processing from the use side. In this interaction, we mean to enable the user to check the rain conditions in different time slots or locations.

IDEA 17: Adding an interaction for both Combining and Mapping from the user side. This idea was inspired by the interaction in Press Light, which is an experimental prototype we made before. In this interaction, the subtle diffused light patterns can turn to clear projected light patterns when the user is pushing the lampshade (see Figure 6.8).

\section{Concepts}




\section{Mapping}

CONCEPT 1.1: The raw data gathered from Buienalarm are filtered into three clusters that respectively indicate: the current rain condition at Eindhoven; the rain condition after 30 mins at 's Hertogenbosch; the rain condition after 90 mins at Utrecht.

CONCEPT 1.2: The data items are normalized to a range of $[0,1]$, in which the numbers of 0 and 1 , respectively, mean the heavy rain or no rain. For the light rain and moderate rain, the threshold numbers are 0.3 and 0.7 .

CONCEPT 1.3: The detailed colour mapping in light and form mapping in shape are presented in Figure 6.9.

Combining

CONCEPT 1.4: The representations of light and shape are combined by means of diffusing and emitting to create a luminous umbrella (see Figure 6.9). On the fabric of this luminous body, the user not only can perceive the diffused light but also can perceive multiple randomly distributed micro brighter points.

CONCEPT 1.5: There are three marks on the carpet to guide the user to stand on them for checking the rain conditions at different locations. These three marks respectively represent: current rain condition at Eindhoven; the rain condition after 30 mins at 's Hertogenbosch; the rain condition after 90 mins at Utrecht.

\section{Rendering}

CONCEPT 1.6: Usually, the luminous umbrella is positioned on the table which is close to the fixed route (see orange arrow in Figure 6.2).

CONCEPT 1.7: The mechanics of changing the luminous umbrella's form follow the traditional ones. However, a motor is connected to the mechanics for triggering the automatic open or close of the umbrella.

CONCEPT 1.8: The luminous umbrella fabric is weaved with the luminous fibres, which are connected to a powerful RGB light source hidden in the handle.

Interactions

CONCEPT 1.9: Positioning multiple sound sensors in the basement to detect how many people are there. If there's nobody in the basement, the information displays will be turned off directly to save energy. While, when someone is entering the basement, the information displays will be automatically turn on smoothly. 
CONCEPT 1.10: Embedding some force sensors on the marks added on the carpet. When the user holds the luminous umbrella and stands on the mark, the information displays will be altered to represent the rain condition in the corresponding time slots and locations. 


\section{Appendix III: The booklet used for evaluation}

This appendix presents the booklet that instructs participants in the evaluation. The first instruction (see the yellow pages) explains the definition of SLS, the SLS Framework, and Exercise 1. The second instruction (see the cyan and deep yellow pages) introduced four SLS design cases, the SLS Interaction Model, and Exercise 2. The last instruction (see the orange pages) introduced the design brief of a hypothetical project, the Design Guidelines, and Exercise 3.

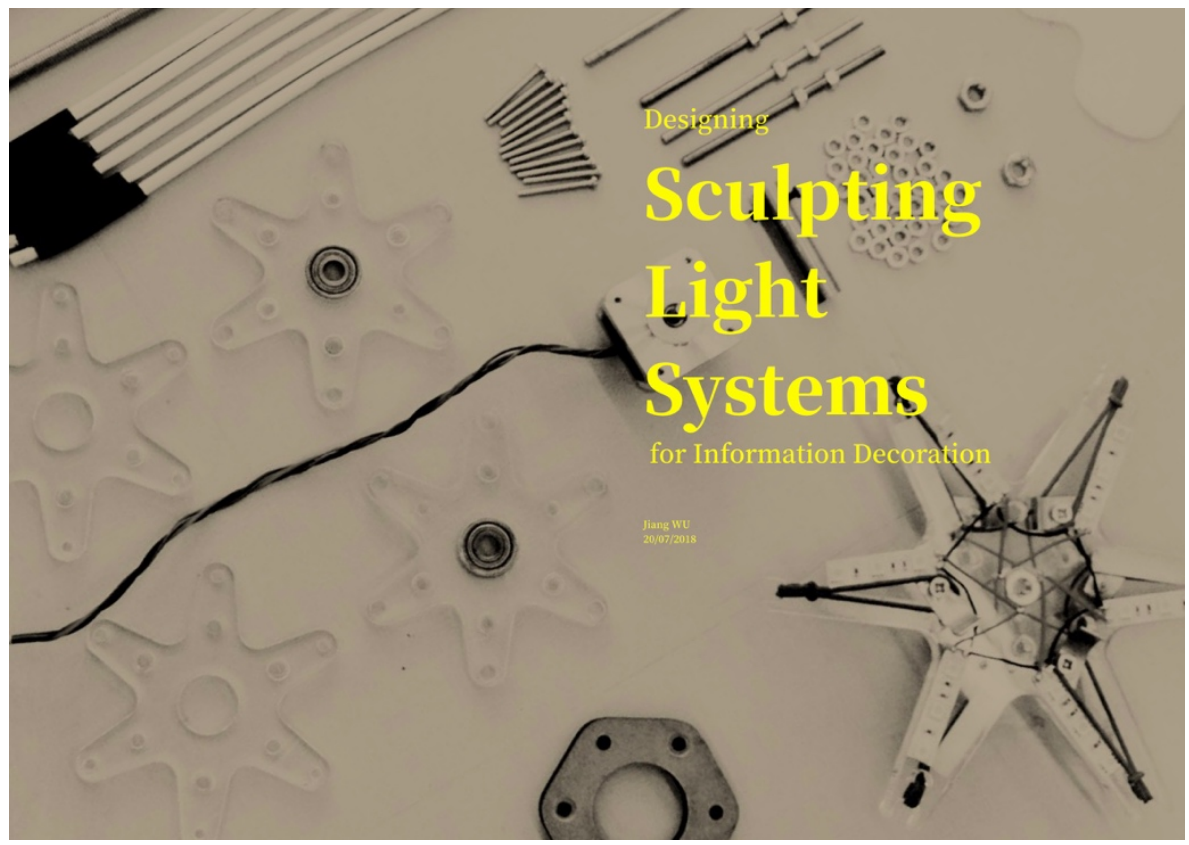




\section{Introduction}

This booklet presents the knowledge we developed for designing SLS (Sculpting Light Systems) for Information Decoration. It includes two main parts: the first 40 pages explain basic of knowledge by means of a Framework and Interaction Model of SLS; the rest 15 pages introduce the use of the basic knowledge by means of Design Guidelines of SLS.

\section{Contents}

Firstly, we introduce the definition of SLS (Sculpting Light Systems) with two existing examples. Then we present a Framework to abstract the essential design elements of SLS and the design alternatives for them. Next, we introduce a specific application of SLS (that is Information Decoration) by our four design explorations. After that, we present an Interaction Model of SLS to show a coherent picture of the possibilities of designing SLS for adaptive Information Decoration. Finally, we propose the Design Guidelines of using our Framework and Interaction Model to develop concepts of SLS for Information Decoration.

\section{Expectations}

Reading this booklet will take you approximately two hours. However, we promise you an innovative perspective to develop interactive lighting, shape-changing interfaces, and ambient displays. Also, we sincerely hope you can spend a little bit more time to give us some valuable feedback by using our knowledge in an evaluation study.

\section{$1 / 55$}

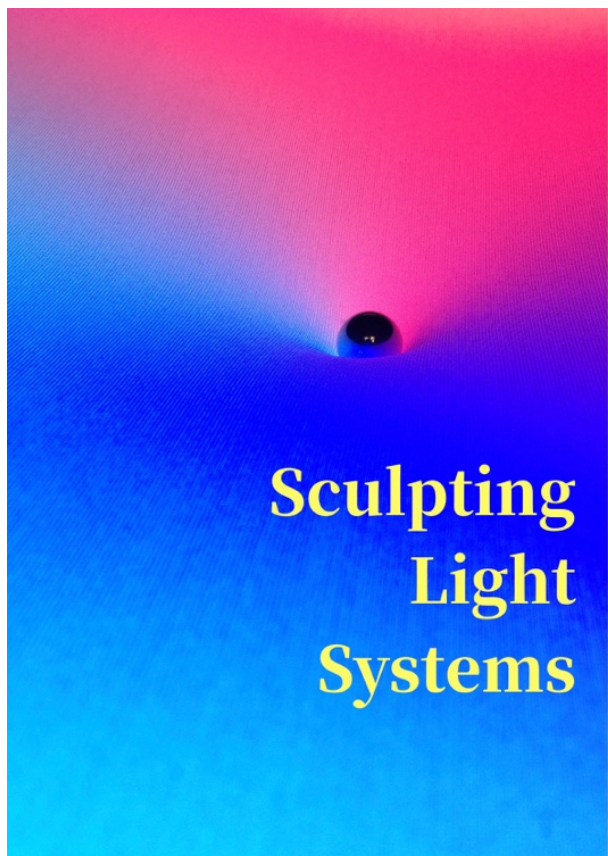

\section{Statement of Informed Consent}

$$
\text { Name: }
$$$$
\text { Age: }
$$$$
\text { Gender: }
$$

This study includes three practice sessions, in which we would like you to respectively use the knowledge of Framework, Interaction Model, and Design Guidelines of SLS. Also, we would like to collect some feedback from you on the usefulness of our knowledge by means of questionnaires, comments, and interviews. During the interview sessions, your speech will be recorded.

\section{Confidentiality}

We will use the data that you provide to us; along with the information we collect from other participants, to conduct research. To ensure confidentiality, we will protect all your privacy in our data.

\section{Freedom to withdraw}

You may withdraw from this study at any time without penalty.

\section{I hereby give consent:}

To use my feedbacks for educational and research purposes. $\square$ YES $\square$ NO

To publicize words from my recordings in written papers. $\square$ YES $\square$ NO

Signature: Date:

\section{Definition}

Sculpting Light Systems (SLS) are lighting systems that combine shape change with light to produce decorative meaningful light patterns. Moreover, SLS can provide multi-modal displays of light and shape, and support the tangible manipulation of light. By SLS, we encourage design practitioners to take full advantage of physicality by combining the natural qualities of light and materials to create an aesthetic, meaningful, and environmentally appropriate lighting systems.
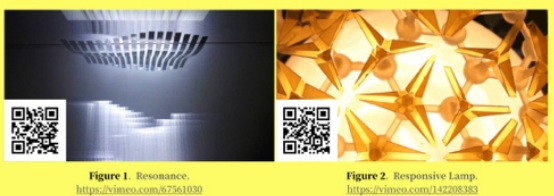

\section{Examples}

In recent years, combinations of shape change and light have emerged increasingly both in the art installation area and the HCI field. For examples, Resonance (see Figure 1) uses a kinetic body of mirrors to produce ever-changing light projections that are cast on the wall; Responsive Lamp (see Figure 2) [1] demonstrates a lamp that closes up its lampshade when it is off and opens up to leak light and create light patterns when it is on.

[1] Yao, L., Ou, J., Cheng, C. Y., Steiner, H., Wang, W., Wang, G., \& Istini, H. (2015, April). Biologic: Conference on Human Factors in Computings Systems $(\mathrm{PP}, 1-10)$, ACY. 


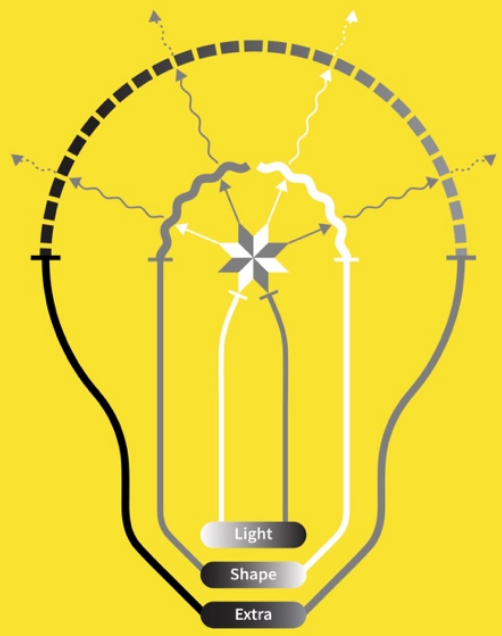

\section{Framework}

To get a basic understanding of the design space for SLS, especially on how to implement SLS in a configurable way, we propose the Framework of SLS. This Framework outlines three categories of design elements to implement SLS: components, attributes, and light patterns. The components concern what types of light sources and physical objects could be used to construct SLS; the attributes involve what kinds of properties can be used to manipulate SLS; the light patterns indicate what means of light expression can be produced by SLS. In the figure on the left page, we integrate these three categories of design element in an abstract illustration:

Components. The white /gray bold wavy lines indicate to the Active/ Passive Objects. Moreover, the gradient bold dash line refers to the Preset Objects.

Attributes. The white/gray/black T-shape lines refer to automatic manual/preset manipulations. The white-and-gray gradient capsules refer to the Light/Shape Attributes for the manipulations of Active/ Passive Lights and Active/Passive Objects. The gray-and-black capsules refer to the Extra Attributes for the manual/preset manipulations.

Light Patterns. The white/gray solid straight arrows refer to the pattern of Initial Light pattern that perceived from the Active/Passive Lights. The white/gray wavy arrows refer to the Sculpted Light patterns that perceived on the Active/Passive Objects. The white/gray dash arrows refer to the Projected Light pattern that perceived on the Preset Objects.

In the next pages, we will introduce these design elements in details. Moreover, we will present the design alternatives for them.

$4 / 5$

Now, we are going to explain the Framework of SLS by introducing its three categories of design elements. To gain better understandings of these design elements, please read the following contents along with watching the videos by clicking the attached websites or scanning the attached QR codes in the examples.

\section{Components}

Firstly, we introduce the components of SLS. Based on different ways of manipulating light sources and physical objects, we identify five alternative components of SLS:

Active Lights refer to light sources that automatically change their light properties. For natural lights (see the daylight in Figure 3), the change of their appearance follows the laws of nature, which cannot be changed by human beings. For artificial light sources, the changes of Active Lights can be manipulated directly by computer systems (see the light performance in Figure 4) or indirectly via tangible interfaces (see the flexible fabric in Figure 6) which are composed by Passive Objects (see the explanation of Passive Objects on the right page).

Passive Lights indicate the light sources that have constant light properties until people change them via an extra independent switch or smart device. For example, to turn on/off a lamp with a switch, or to change the color of Phillips Hue via a smartphone.

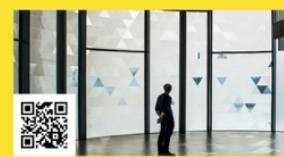

Figure 3 . Shade.

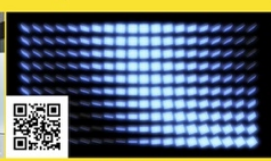

Figure 4 Kinetic Wall of thight

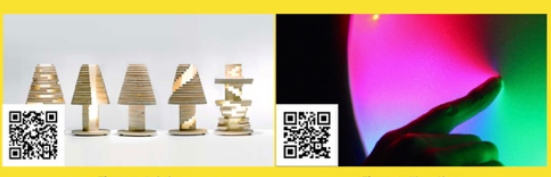

Figure 5 thabele.

Active Objects are shape-changing objects that can automatically change their physical shapes. Such an automatic shape-changing can be actuated by a computer program (see the kinetic mirrors in Figure 1 on page 3). Also, the shape change of active objects could be animated by specific properties of the object's material. For example, Responsive Lamp (see Figure 2 on page 3) [1] can close/open up its lampshade with the decrease/increase of its surrounding temperature.

Passive Objects are shape-changing objects which physical shapes are manually changed by people's continuous touch only. The manipulations of Passive Objects can naturally produce ever-changing light patterns (see the disassembling modules in Figure 5). Also, the Passive Objects can be implemented as a tangible interface (see the flexible fabric in Figure 6) for manipulating the light properties of the light sources to create unusual light patterns.

Preset Objects involve the non-shape-changing objects which are preset with some constant physical properties. They are implemented to show light patterns (see the shadows on the floor in Figure 3). Besides, it is also possible to implement the Preset Objects to trigger the automatic manipulations of Active Lights/Objects. For example, in the case of Reflective Clock (see page 24), the light pattern changes its color to red gradually when approaching the red sticky-notes. 


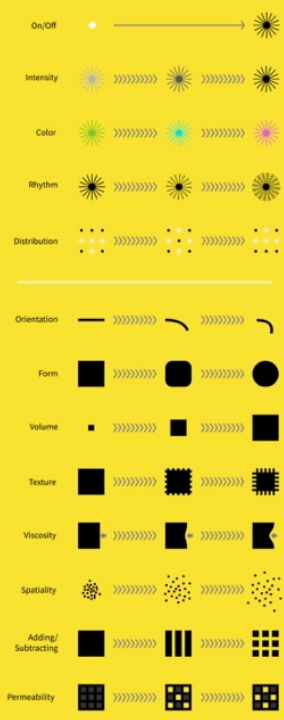

$7 / 5=50$

\section{Attributes}

Secondly, we introduce the alternative attributes that can be applied to manipulate the components of SLS.

Light Attributes are used to manipulate the light sources. There are multiple basic Light Attributes (see the top figure on the left page) which include: on/off, intensity, color, rhythm, and distribution. These attributes can be combined for the manipulation of the Active/Passive Lights. For example, in the case of Kinetic Wall of Light (see Figure 4 on page 5), you can see various light controls with different combinations of light attributes.

Shape Attributes refer to the properties of physical shape change. According to the framework of Rasmussen [2], there are eight types of shape change (see the bottom figure on the left page): orientation, form, volume, texture, viscosity, spatiality, adding/subtracting, and permeability. These Shape Attributes can be integrated into a specific shape change. For example, Responsive Lamp (see Figure 2 on page 3) presents a permeability changing lampshade. However, such a shape display is realized actually by changing the orientations of its shapechanging elements.

Extra Attributes indicate the attributes that are used to preset the Preset Objects to some constant state such as position, material, pattern, texture, scale, etc. For example, we added some scales on the white-board in the case of Reflective Clock (see page 24) to support clear information presentation. Also, the Extra Attributes can be involved for manually manipulating the Preset Objects. For example, in the case of Reflective Clock (see page 24), the user places the stickynote on the corresponding scales. [2] Rasmussen, Mtajken K, et al. "Shape.changing interfaces: a rewiew of the design space and open
research questions." Proceedings of the SIGCHI Conference on Human Factors in Computing Systems. ACM, 2012

\section{Light Patterns}

Thirdly, we introduce the light patterns that the user can perceive in SIS. According to the physical process taking place between light sources, physical objects, and users, we define three classes of light patterns based on the three phases of light paths, which are Initial Light, Sculpted Light, and Projected Light. Moreover, we specify these light patterns by six means of light-object interactions in below.

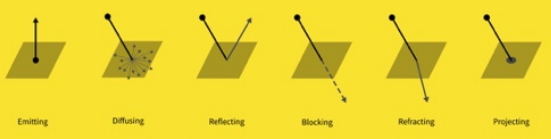

Initial Light is only perceived directly from the light sources as emitting patterns.

Sculpted Light can be perceived either from the light sources or the physical objects, depending on the point of the light paths at which the physical objects are deploved. If the physical objects are light fixtures, which are deployed at the start point of light paths, then the Sculpted Light will be perceived from the light sources as emitting patterns (see the kinetic light displays in Figure 4 on page 5). If the physical objects are deployed halfway of the light paths, then the Sculpted Light will be perceived from the physical objects. In this case, the Sculpted Light can be diffused patterns (see the lamp body in Figure 7), reflected patterns (see the light reflections on the kinetic mirrors in Figure 1 on page 3), blocked patterns (see the light gaps on the glass in Figure 5 on page 6), and refracted patterns (see the light refractions on the water in Figure 8).
Projected Light is only meant to be perceived on the Preset Objects which are intentionally fixed to show the Sculpted Light as projected patterns. They could be shadows or reflected/refracted projections. For examples, the shadows on the floor (see Figure 3 on page 5), the reflected projections on the wall (see Figure 1 on page 3 ), and the refracted projections (see Figure 8).

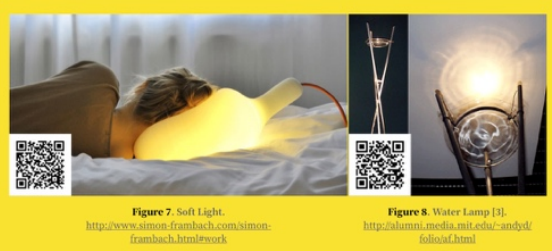

\section{Styles of SLS}

In addition to the three categories of design elements, there are four typical styles of SLS in the Framework of SLS. They are linked to the four basic combinations of Active/Passive Lights and Active/Passive Objects. If the Preset Objects are used depends on whether the Projected Light will be emphasized or not. These four styles of SLS can be applied to four basic scenarios.

[3] Dahley, Andrew, Craig Wisneski, and Hiroshi Ishii. "Water lamp and pinwheels ambient projection of digital information into archincer 


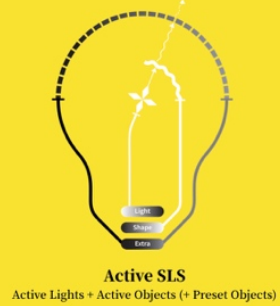

Active SLS are implemented by combining Active Lights with Active Objects to present lively expression of light and shape. For example, in physical movement of its light panels (Active Objects) and the light of the attached LEDs (Active Lights) are manipulated by a computer program synchronously. Also, Active SLS can involve Preset Objects to show or manipulate the Projected Light. For example, in the case of Shade (see Figure 3 on page 5), the designers mean to not only present the flowing light gaps on the glass facade but also the shadows on the floors (Preset Objects). For another example, in the case of Reflective Clock (see page 24), the color of its reflected projections changes according to the color and position of the added sticky-note (Preset Objects.

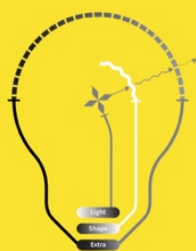

Objects-driven SLS

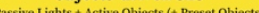

Objects-driven SLS are implemented by combining Passive Lights with Active Objects. This type of SIS could be applied to a context where the initial light is not suggested to change automatically, for example, the functional lighting in a working context. Usually, Objects-driven SLS functional lighting in a working context. Usually, Objects-driven SLS
involves Preset Objects to show Projected Light. For example, Water Lamp [3] (see Figure 8 in page 10) deploys a passive light source to project the active physical ripples, which are actuated by three small solenoids tapping on a small basin of water, on to the ceiling (Preset (he Preset Objects can also be involved to manipulate the Projected Light. For example, what if the Water Lamp can change the frequency of the solenoids tapping on the water when the user places it in a different room (Preset Objects).

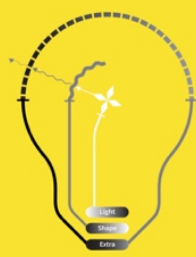

Lights-driven SLS Active Liths + Passive Objects (+ Preset Objects)
Light-driven SLS are implemented by combining Active Lights with Passive Objects. In this case, the Passive Objects are integrated with the lighting interfaces to support the user with tangible manipulation of light. For example, Time Glow (see page 25) enables the user to manipulate the light representation of time by poking in or pulling out its elastic lampshade (Passive Objects). Also, Lights-driven SLS can involve Preset Object to show the Projected Light. For example, the Press Lights (see Figure 10 on page 16) implement a whiteboard (Preset Objects) to show the light projections, which are produced by a combination of a flexible frame (Passive Objects) and 16 LEDs (Active Lights).

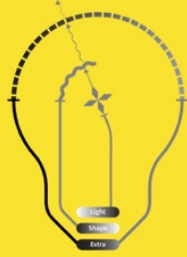

Passive SLS

Passive Lights + Passive Objects ( + Preset Objects)

Passive SLS are implemented by combining Passive Lights and Passive Objects. This style of SLS enables users to create or explore everchanging light expressions by manually manipulating the light sources and physical objects. For example, Babele (see Figure 5 on page 6 ) enables the user to disassemble the form of its lampshade (Passive Objects) manually to create different patterns of light gaps. While the light source (Passive Lights) of Babele is kept to a constant state. Also, Passive SLS can involve Preset Objects to show the Projected Light. A classic example of that is the Shadow Play [4]. [4] The definition of Shadow play from wilipedia: Shadow play also known as shadow puppetry, is (shadow puppets) which are held between a source of light and a translucent screen or scrim. The cut-out shapes of the puppets sometimes include translucent color or other types of detailing. Various effects can be achieved by moving both the puppets and the light source. A talented puppeter can make the figures appear to walk, dance, fight, nod and laugh. 
In the previous pages, we have introduced the Framework of SLS, which presents a systematic way to implement SLS with three categories of design elements: components for construction, attributes for manipulation, and light patterns for expression. Also, the Framework of SLS abstractly indicates four typical styles of SLS, which can fit into different scenarios.

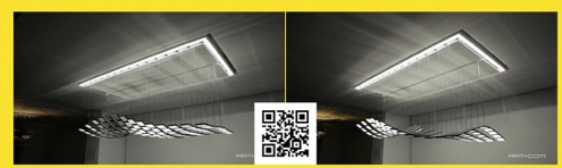

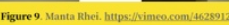

In the actual design of SLS, all the design elements mentioned above can co-occur. It means that it is possible to combine multiple styles of SLS in one design case. For example, Manta Rhei (see Figure 9) combines two styles of SLS with one kinetic sculpture (Active Objects). The one is Active SLS which changes the intensities of the attached OL.EDs (Active Lights) on the front side of the kinetic sculpture synchronously to produce emitting patterns. The other one is Objectsdriven SLS which uses the back side of kinetic sculpture to reflect the light from the ceiling lamps (Passive Lights) to produce projected patterns on the ceiling ( Preset Objects). These two styles of SIS together create a dramatic light sculpture.

\section{$15 / 5$}

\section{Press Light}

https://vimeo.com/274491229

Which styles of SLS are implemented in this example?

Lights driven SIS

Active Lights + Passive Objects + Preset Objects

What components and attributes are implemented in this example?

Active Lights and Light Attributes:

Press Light attaches 16 LEDs on a flexible fram

the colors and intensities of every individual LED via

a tangible interface which is composed of the flexible frame.

Passive Objects and Shape Attributes:

Press Light uses a flexible frame whose form

can be changed by users' continuous pressing.

Preset Objects and Extra Attributes:

Press Light uses a whiteboard on its bottom to show the light projection. What light patterns are implemented for expression in this example?

Projected Patterns:

Press Light uses a whiteboard to show

the emitting patterns of the 16 LEDs as soft projected patterns.

\section{Practice 1}

So far, we assume that you have understood the knowledge we have introduced in the previous pages. Now, we would like you to use this knowledge in a practice session to describe an example of SLS (see Figure 11). More specifically, you will specify the implemented design elements in this example through answering three questions on page 18.

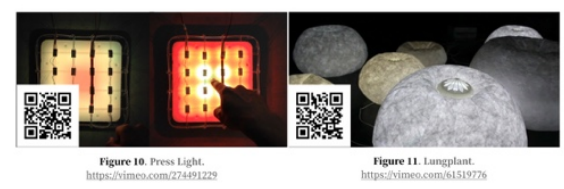

Before you start, please review how we describe another example (see Figure 10) of SLS on page 17. The point is to not only indicate what design elements are implemented but also explain how these design elements are implemented.

In this practice, we expect your description is based on a full understanding of the example in Figure 11. So, please make sure that you have watched the video demonstration of this example before you start with answering the questions on page 18 .

$16 / 5$

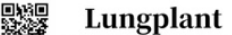

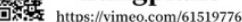

Which styles of SLS are implemented in this example

What components and attributes are implemented in this example? 


\section{Questionnaire \&Comment 1}

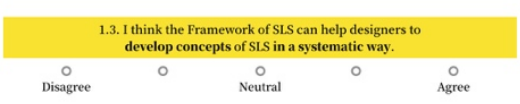

After the practice session, we would like to collect some feedback from you on the value of our Framework of SLS. Please rate in the below questionnaire. Also, we will appreciate it if you can leave a comment to explain each of your rates with a few words.

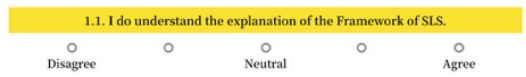

1.4. 1 think the Framework of SIS can help designers to generate many alternative ideas to compose the concepts of SLS.

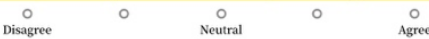

1.2. I think the Framework of SLS is understandable for other designers.

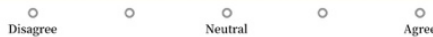

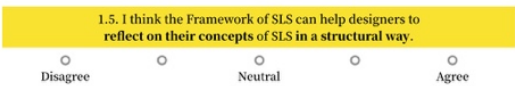

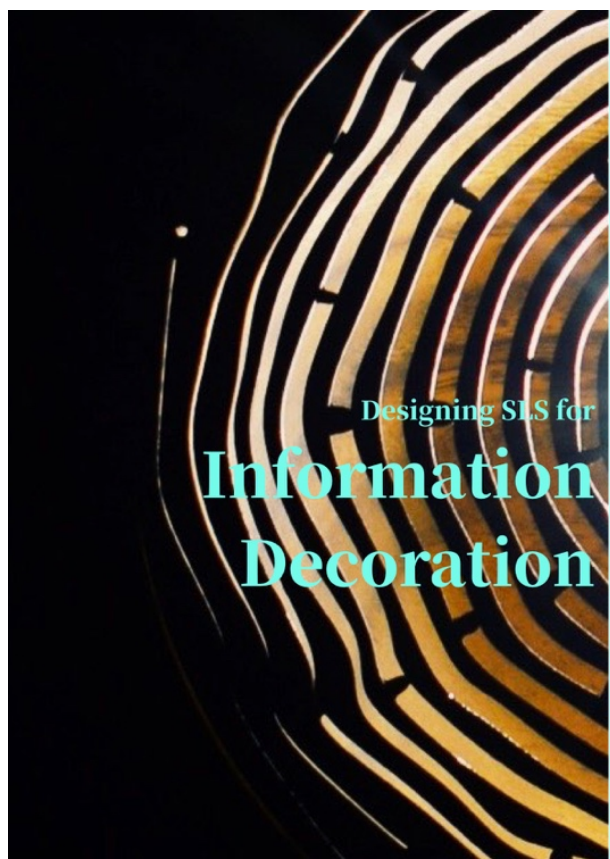

Motivation

SLS combine light and shape change to provide multi-modality displays of light and shape, and it supports tangible manipulation of light. On the one hand, the multi-modality of light and shape could be aesthetically coupled with digital information in a peripheral and subtle way, while, on the other hand, the tangible controls of light might evoke people to interact with light and information in a natural and intuitive way. Therefore, we focus our study of SLS on the specific field of Information Decoration [5]. More specifically, we explore the possibilities of designing SLS for Information Decoration which are not only aesthetically pleasing to the user but also interactively adaptive to the context.

\section{Design Explorations}

To explore this specific design space of designing SLS for Information Decoration in practice, we developed four design cases which will be presented in the next four pages. These design cases are implemented by three different styles of SLS, which are Active SLS, Objects-driven SLS, and Lights-driven SLS. We skip the Passive SLS because there are already many existing examples of that, for instance, shadow signage. Our design cases show different possibilities of integrating SLS into a context by enabling the user to alter the Information Decoration.

In the next four pages, you will read the descriptions of the four design cases. Also, please check the video demonstrations of the design cases for detailed information by clicking the attached websites or scanning the attached $\mathrm{QR}$ codes.

15) Ergen, Berns and Koert Van Nenswoort. "Making sense of what is going on 'around' Designing emvironmental awareness information displass." Awareness Systems. Springer, London, 2009.99-124.

21/5: 


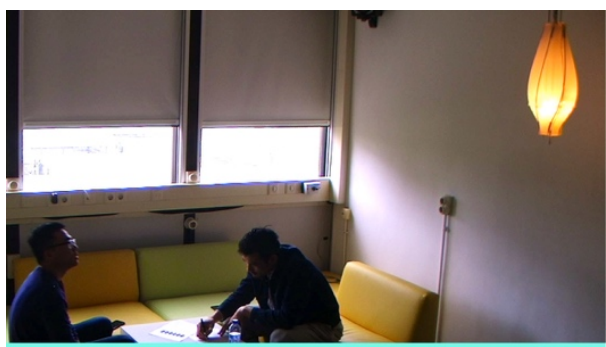

哭管回 Timer Lamp

https://vimeo.com/274437412

Timer Lamp is an example of Active SLS for Information Decoration. It is a fusiform ceiling lamp that consists of a deformable lampshade (Active Objects) and an up-and-down moveable light source (Active Lights). Timer Lamp produces beautiful and subtle diffused patterns (Sculpted Light) that fade out from the fullest part to both ends of the lamp body. With an Android App, users can set the duration of the meeting and start Timer Lamp.

By this example, we demonstrate a lively multi-modal display of light and shape, in which the light change and shape change complement each other to represent one piece of information in a rich way. Also, we show the possibility of enabling the user to preset the Information Decoration of SLS by using an additional independent interface.

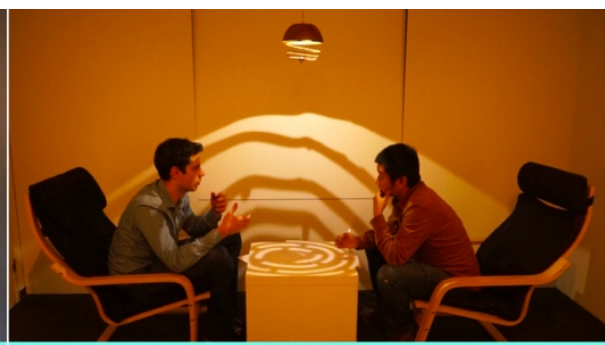

Timer Shade

https://vimeo.com/274438399

Timer Shade is an example of Objects-driven SIS for Information Decoration. It possesses a flexible, moveable coiled bottom cap, and a power LED (Passive Lights) with a constant brightness. With the uniform up and down movement of the coiled cap (Active Objects), not only the shape of the lamp body changes but also the shadows (Projected Light) cast on the table (Preset Objects) will change. Timer Shade enables the user to set the duration of the meeting by pulling down the coiled bottom cap to positions corresponding to a specific meeting time, respectively.

By this example, we demonstrate the possibility of employing the surroundings of the user as Preset Objects to show the decorative meaningful light patterns. Also, we show the potential of enabling the user to preset the Information Decoration by manually manipulating the Active Objects before it starts.
22/

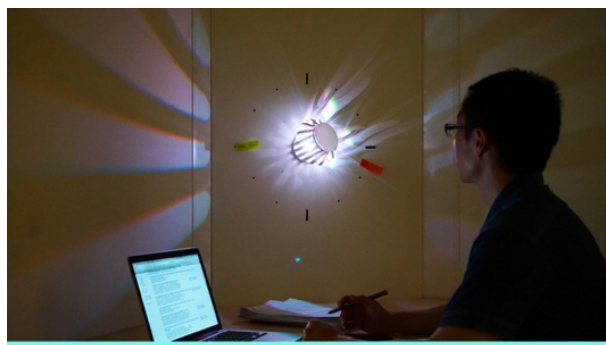

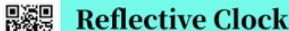

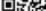

huttps://vimeo,com/274517223

Reflective Clock is another example of Active SLS for Information Decoration. It consists of a flexible cage (Active Object), which is made of mirror sheet, an RGB LED (Active Lights) as the only light source for this lamp, and a scaled whiteboard (Preset Objects). In general, Reflective Clock produces subtle reflected light projections (Projected Light) that cast on the board to show general time information. The user can place his/her sticky-notes (Preset Objects) to the corresponding scales on the board as physical reminders. When the light projections are approaching the sticky-note, the color of it will gradually change to the color of the note.

By this example, we demonstrate the possibility of presetting the Preset Objects with some Extra Attributes to better present the information representation. Also, we show the potential of enabling the user to manipulate the Preset Objects to alter the notification level of the Information Decoration.

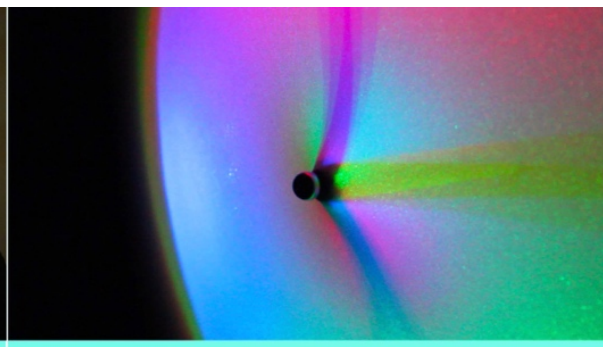

Time Glow

https://vimeo.com/275524164

Time Glow is an example of Lights-driven SIS for Information Decoration. It possesses a fabric cover (Passive Objects) and 60 RGB LEDs (Active Lights), which are distributed along the circle inside the cover. Time Glow can produce two different kinds of diffused pattern: one is colored shadows when the user pulls out the center of the cover; the other is the color wash light when the user pokes in the center of the cover. These two kinds of light pattern represent real-time information in two different means: the colored shadows (produces by three RGB LEDs) show clear ticks; the color wash light (produces by 15 RGB LEDs) present the subtle flow of time.

By this example, we demonstrate the possibility of enabling the user to alter the representation fidelity of Information Decoration by manipulating the Passive Objects. 


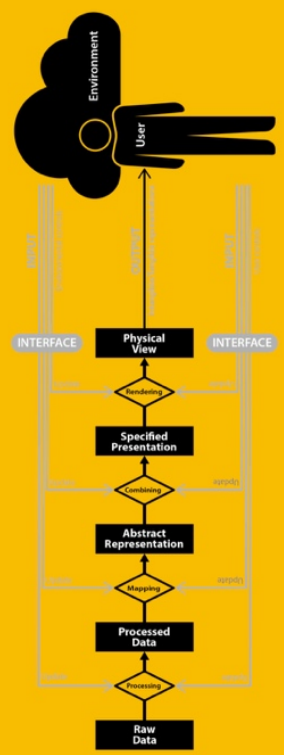

\section{Interaction} Model

In the previous pages, we have present the possibilities of applying SLS for adaptive rather than perceive-only Information Decoration systems. To understand this particular design space systematically, we propose an Interaction Model of SLS, which provides designers with a coherent picture of what is possible when developing adaptive SLS for Information Decoration.

This interaction model consists of two main parts which are Information Flow (black arrows in the figure on the left page) and Interaction Loops (gray arrows in the figure on the left page). In each of these two parts, we specify what design elements designers need to define when developing adaptive SIS for Information Decoration. The Information Flow describes the process of how to transform Raw Data into an informative SLS through five stages (black blocks in the figure on the left page) and four transformation steps (black diamonds in the figure on the left page). The interaction loops illustrate how the Information Flow can be altered by manipulating the four transformation steps in four types of interaction loop. These interactions could be triggered by either user controls or environmental controls.

In the next pages, we will explain our Interaction Model by introducing the design elements mentioned above. Moreover, we will indicate some design alternatives for these design elements.

26/

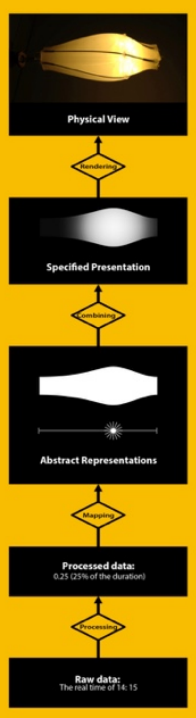

Rendering

Presentation of Timer Lamp is finally implemented as a ceiling lamp in a meeting room. It consists of a nexible framework, a fabric lampshade, and a LED-equipped pad. Also, we imploy a DC motor to actuate the according of the LFD.equipped framework and the fabric lampshade change their shapes.

Combining.

The abstract representations of Timer Lamp are combined into the diffused light patterns, which look like a floating up light bubble which symbolizes a paronomasia of "time is being up.

Mapping.

The Processed Data is mapped to two different abstract representations: one is the movement of the Active Lights; the Objects.

Processing.

The Raw Data of "14:15" (real time) is 25 ," which means 25 of the meeting time has passed.

\section{Information Flow}

Firstly, we introduce the Information Flow of SLS for Information Decoration. More specifically, we explain the four steps and the five stages in the process of data transformation. Also, we demonstrate an example of the Information Flow (on the left page) in the concept of Timer Lamp (see page 22).

Processing. At first, the Raw Data is transformed into a format of Processed Data that is suitable for information representation.

Mapping. Then, the Processed Data will be mapped to the Abstract Representation through a Mapping step. In this transformation step, the dimensions of the Processed Data will be assigned to the scales of the variable attributes used for the abstract representations. In SLS, the Abstract Representation refers to the light change of Active/Passive Lights and the shape change of Active/Passive Objects.

Combining. The Combining step aims to draw a scheme of how the Abstract Representations will be combined into a Specified Presentation. The core of this scheme is applying the light-object interaction to produce light patterns. Sometimes the Preset Objects might be involved. Moreover, the Combining step involves four operations to embody the light patterns in a symbolic and decorative way. These operations are deploying and materializing the SLS components, specifying the variable attributes of every Abstract Representation, and optimizing the non-coding properties of SLS to facilitate an aesthetically pleasing Specified Presentation.

Rendering. At last, the Rendering step brings the Specified Presentation into reality as Physical View that the user can perceive and interpret. This step is about realizing the Specified Presentation in the aspects of technology, materials, and fabrication. Also, this step concerns how to integrate the Specified Presentation into the given context. 


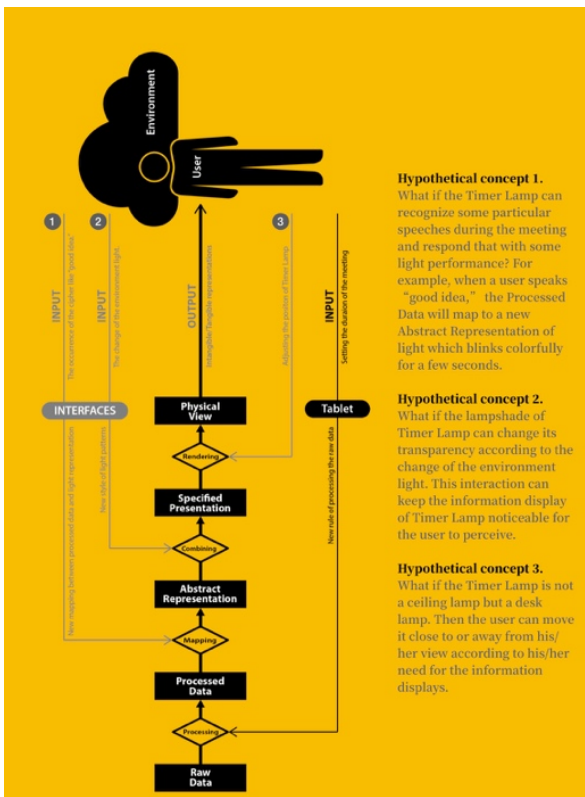

29/

\section{Interaction Loops}

Secondly, we introduce the Interaction Loops of SLS for Information Decoration. More specifically, we explain four types of interaction loop that respectively alter the four data transformation steps. Also, we demonstrate these interaction loops with either the existing concept or the hypothetical concepts we came up with in the context of Timer Lamp (see page 22)

Interaction Loops for Processing. This interaction alters the data Processing to meet different demands for information element or capacity without directly affect other data transformation steps. For instance, in the case of Timer Lamp, we enable the user to set the duration of the meeting with a tablet.

Interaction Loops for Mapping. The purpose of this interaction is to adapt the information decoration to the context according to the user' $s$ different needs for representation fidelity or notification level. It alters the Mapping step without directly affect other data transformation steps. The example can be seen in our hypothetical concept 1 on page 29.

Interaction Loops for Combining. This interaction aims to alter the presentation quality or notification level of the information decoration. It alters the Combining step without directly affect other data transformation steps. In this case, the abstract representations could be recombined by applying a new way of deployment, materialization, or specification. The example can be seen in our hypothetical concept 2 on page 29

Interaction Loops for Rendering. This interaction changes the final Physical View of SLS by altering the set-up of the Specified Presentation. It alters the Rendering step without directly affect other data transformation steps. The example can be seen in our hypothetical concept 3 on page 29 .

$30 /$

\section{Design Alternatives}

At last, we summarize the design alternatives of developing SLS for Information Decoration. The design alternatives for the Abstract Representation, Specified Presentation, and Physical View are from our Framework of SLS. While, the design alternatives for the Interaction Loops are some available interaction techniques for the user controls, environmental controls, and interfaces.

Abstract Representation. The main issue of designing Abstract Representation is selecting the components and the attributes of SLS. According to the Framework of SLS, there are four alternative components (see the Active/Passive Lights/Objects on page 5-6) and various alternative attributes (see the Light/Shape Attributes on page 7-8) for Abstract Representation. Also, the four styles of SLS (see page 11-14) can be involved in developing the Abstract Representation.

Specified Presentation. The Specified Presentation draws a scheme of how the design elements in the Abstract Representation will be combined. The core of this scheme is producing meaningful light patterns (see Initial/Sculpted/Projected Light on page 9-10). Based on the Framework of SLS, there are six fashions of light patterns by six types of light-object interactions (see the figure on page 9). Moreover, in some cases, the Preset Objects (see page 6) could be involved. So the Specified Presentation also might need to concern the specification of the Preset Objects with some Extra Attributes (e.g., spatiality, material, pattern, texture, scale, etc.) to better present the light patterns.

Physical View. Designing the Physical View relates to the realizations of technology, material, and fabrication. While integrating the Specified Presentation into the context should be more concerned first. There are two alternative ways to do so: using existing light sources as Active lights (e.g. sunlight) or Passive Lights; using environmental objects as Preset Objects to show or manipulate the Projected Light
User controls. There are two types of user controls can occur in the interaction loops from the user side. The one is the explicit controls which are caused by the user that intentionally alters the information displays; the other is the implicit controls which are triggered by the user that unintentionally changes the information displays.

Environment controls. The environment controls are meant to adapt the information displays of SLS to the dynamically changing environment. There are two kinds of such dynamic changes: climate and context. The climate refers to the light, sound, energy consumption, and even private security in the environment. The context means to the activity, event, atmosphere, and even schedule of the user/users in the environment.

Interfaces. This taxonomy concerns the types of interface to transform the user/environmental controls to the inputs for altering the information displays of SLS.

From the User side, there are three types of available interfaces: GUI, TUI, and NUI. By mean of GUI, such an interface indicates an additional digital device like a smartphone or tablet. TUI merge the user control and Active/Passive/Preset Objects of SLS into the same interaction space and in doing so provides additional haptic feedback. NUI enable the user to control SLS in a natural way without using artificial control devices but by sensing the user' s activities (e.g. Proxemic interaction), movements (Gesture Interaction), and voice (Speech Interaction). Both of the TUI and NUI can be used in either explicit or implicit interaction.

From the Environment side, the interfaces are more about the sensors that are used to detect the dynamic changes in the environment. For instance, to realize the interaction loop in the hypothetical example 2 showed on page 29 , some photosensitive sensors are needed. 


\section{Practice 2}

Please specify the Raw Data, the Processed Data and the Processing step in the Information Flow of Time Glow.

$21: 15: 41 \rightarrow \mathrm{h}(46) ; \mathrm{m}(15) ; \mathrm{s}=(41)$

Please specify the Abstract Representation and the Mapping step in the Information Flow of Time Glow.

In this practice session, you will use our Interaction Model of SLS to describe the concept of Time Glow which has been introduced on page 25. Based on that, we would like to challenge you to develop a new concept of Time Glow by considering other design alternatives that are provided by our Interaction Model.

For describing the original concept of Time Glow, please finish the following three tasks:

At first, please specify the Information Flow of Time Glow by filling the gaps on page 34. In this specification, you will take the Raw Data of "21:15:41" as an example. We have filled the first gap. For the rest three gaps, you can follow the way we describe the Information Flow of Timer Lamp on page 27.

- Then, please highlight the interaction loop of Time Glow in the figure on page 35 .

At last, please specify the input, interface, and update of that interaction loop by filling the gaps on page 36 .

For developing a new concept of Time Glow, please finish the following two tasks:

At first, please highlight the interaction loop in the new concept of Time Glow on page 37.

- Then, please specify the inputs, interfaces, and updates of the newly added interaction loop by filling the gaps on page 38 .

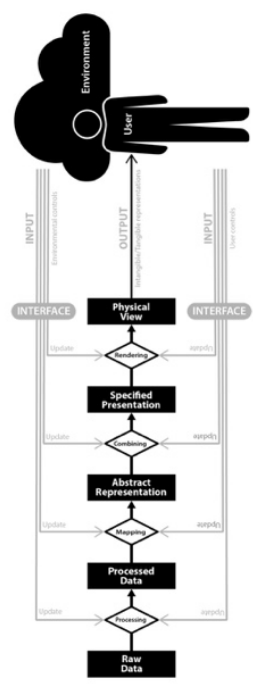




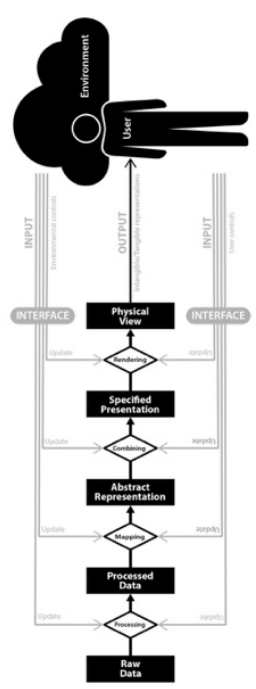

$37 / 55$

\section{Questionnaire \&Comment 2}

$38 / 5$
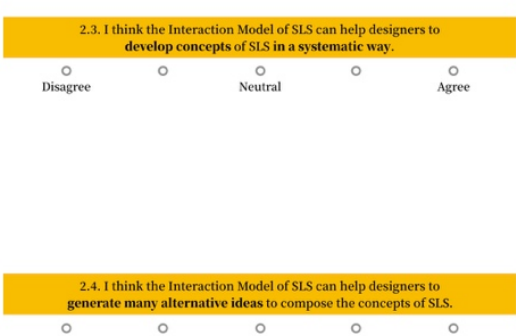

○

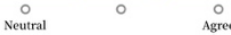

\subsection{I think the Interaction Model of SLS is understandable for other designers.}

$\begin{array}{cccc}\circ & \circ & \circ & \circ \\ \text { Disagree } & \text { Neutral } & \circ \\ \text { Agree }\end{array}$

2.5. I think the Interaction Model of SLS can help designers to reflect on their concepts of SLS in a structural way.
Disagree
$\stackrel{\circ}{\text { Neutral }}$
$\stackrel{\circ}{\text { Agree }}$ 


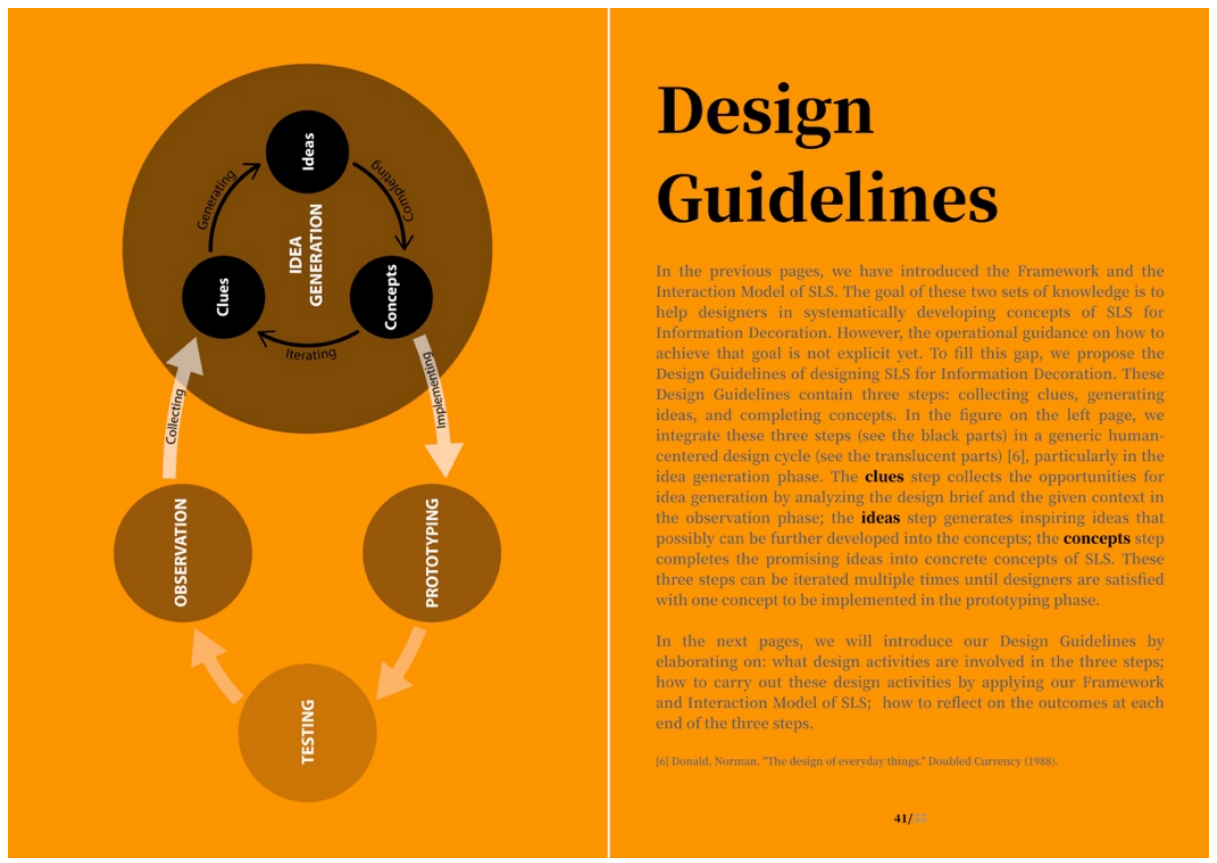

Meanwhile, we would like you to practice our Design Guidelines in a hypothetical project. In this project, we challenge you to propose a creative but affordable concept of SIS that can support people in the basement of Laplace building to be aware of the local rain forecast. The context information of this hypothetical project is described in belor

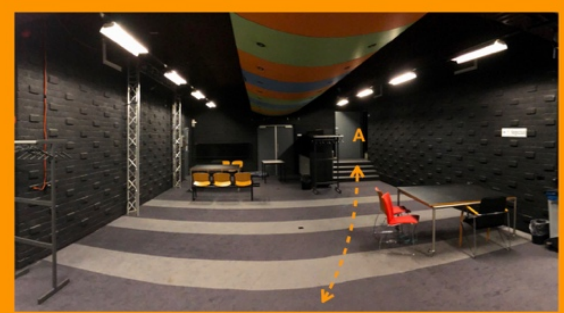

Figure 12. The open space in the basement of LaPlace building.

The SIS will be implemented in the open space in the basement of LaPlace building (see Figure 12). This space is usually quiet unless there is a lecture in the classroom (the Point A in Figure 12). The students might come from different faculties in the university. Their activities in the basement happen on the path (the orange curve in Figure 12) between the ground floor and the classroom. The students do care about the rain condition, especially when they are about to leave the building in 30 mins. However, it is impossible for them to check the rain condition by glancing through the glass as usual. So, they can only check that with an app named Buienalarm, which is very popular in the Netherlands.

\section{Collecting Clues}

The first step is about collecting clues to develop concepts of SLS for Information Decoration. These clues show opportunities for specifying the design elements of our Interaction Model. To collect such clues, we recommend designers to analyze the design brief or to observe the context by carrying out seven design activities presented in the table below.

\section{Collecting Clues to Raw Data by:}

Looking for possible information sources.

\section{Collecting Clues to Processing and Processed Data by:}

Investigating the specific information that might interest the user most.

Collecting Clues to Mapping and Abstract Representation by:

Looking for opportunities for representing the information by means of light change and shape change.

\section{Collecting Clues to Combining and Specified Presentation b}

Looking for opportunities for presenting the information with light patterns in an aesthetic and meaningful way.

\section{Collecting Clues to Rendering and Physical View by:}

Looking for opportunities for integrating the information decoration into the physical environment.

Collecting Clues to Interaction Loops from the user side by:

Looking for opportunities for adapting the information decoration to the user' s activities.

Collecting Clues to Interaction Loops from the environment side by:

Looking for opportunities for adapting the information decoration to changes in the environment. 
Now, we would like you to collect clues for the hypothetical project. Instead of observing the context by yourself, please analyze the design brief and context page 43. Once you found a clue, please describe it and also map it to the relevant design element in our Interaction Model on this page. For example, we map a clue of "applying 'Buienalarm' for the data source" to Raw Data.

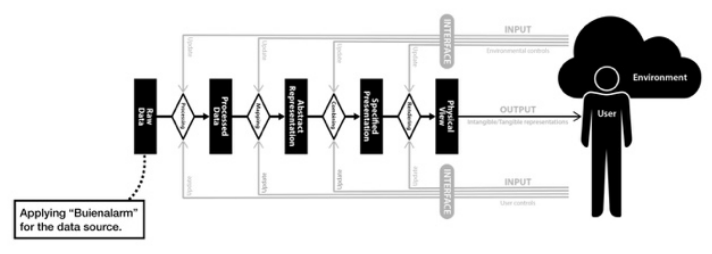

At the end of this step, we recommend designers to reflect on their collect clues to select the useful ones that can support idea generation in the next step. Such a reflection can follow two criteria presented in the table below.

\section{Quantity}

Do the collected clues involve all the design element of our Interaction Model? If not, why?

\section{Quality}

Are the collected clues objective? If not, what are the values of the subjective clues?

Now, please reflect on your collected clues and highlight the useful ones on page 44

\section{Generating Ideas}

The second step is about generating ideas based on the collected clues. These ideas specify the information (re)presentations of SLS in terms of Abstract Representation (see the definition of Processing on page 28), Specified Presentation (see the definition of Combining on page 28), and Physical View (see the definition of Rendering on page 28). Also, these ideas can indicate some possible Interaction Loops (see page 30). To generate such ideas, we recommend designers to carry out four clusters of design activities in the table on the right page.

In this step, designers can carry out these four clusters of design activities in any order. While, in each of the four clusters of design activities, we recommend designers to first define what design alternatives (see page 31 and 32) of developing SLS for Information Decoration can be used, then to search for inspirations freely to specify the selected design alternatives as ideas.

$$
45 /
$$

\section{Generating ideas for Abstract Representation b}

- Selecting the possible styles of SLS that can fit in the given context. - Selecting the suitable types of Light Attribute or Shape Attribute for the SLS components for information representation.

- Explaining the meaning of the information representation.

\section{Generating ideas for Specified Presentation by:}

- Selecting the possible light patterns that are suitable for information presentation in the given context.

- Depicting the expression and meaning of the light pattern.

- Drawing a scheme of what specific shape changes and light changes are needed to produce the light pattern.

- Considering the use of Extra Attributes to optimize the information presentation that involves Preset Objects.

\section{Generating ideas for Physical View by:}

- Thinking about utilizing the existing light sources in the environment as the Active Lights or Passive Lights.

- Thinking about utilizing the existing physical objects in the environment as the Preset Objects.

- Trying out the light patterns with simple experiments in reality or by computer simulation.

\section{Generating ideas for Interaction Loops by:}

- Selecting the Interaction Loops that are worth to add.

- Thinking about what possible Controls are needed to trigger the added Interaction Loops.

- Selecting the suitable interaction techniques to support the added Interaction Loops.

- Thinking about integrating the Preset Objects with the Interfaces in the added Interaction Loops.

$$
\text { 46/ }
$$


In this practice, you will generate ideas for the hypothetical project by following the design guidelines we have introduced on page to. On describe/draw it and also map it to the relevant design element in our Interaction design element, while the depiction of the idea should indicate which design alternatives are used. For example, we map an idea of "using the Active Lights to represent different rain conditions with different colors. Representation.

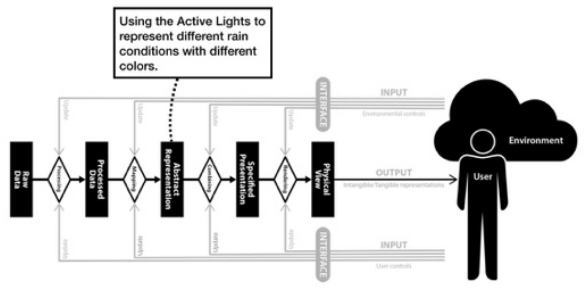

At the end of this step, we recommend designers to reflect on their generated ideas by evaluating the qualities of their ideas in four criteria that are presented in the table below. Such a reflection can help designers to select the promising ones that can be further completed into a concept in the next step.

\section{Subtle.}

The information (re)presentation should be subtle without demanding focused attention from the user.

\section{Non-intrusive.}

The information (re)presentation should be non-intrusive but provides an appropriate level of notification according to the different importance of the information

\section{Aesthetically pleasing.}

The information (re)presentation should be aesthetically pleasing rather than being useful only.

\section{Well-integrated.}

The information (re)presentation should seamlessly merge with the environment. Moreover, the interaction should be appropriately integrated into the context.

Now, please reflect on your generated ideas and highlight the promising ones on page 47 .

\section{Completing Concepts}

The last step is about completing the generated ideas to the concrete concepts of SLS. In this step, designers will combine the generated ideas. Also, designers need to specify the data transformation steps and the interaction loops with detailed definitions. To do so, we recommend designers to carry out four clusters of design activities in the table belo

\section{Completing for Mapping by:}

Defining the specific mappings between the information changes and the variable attributes of manipulating the Active/Passive SLS components.

\section{Completing for Combining by:}

- Defining the deployments of all the employed SLS components to realize the desired light patterns.

- Defining the material properties of all the employed SLS components to realize the desired light patterns.

- Defining the specific variables of every Abstract Representation.

- Defining the specific attributes of the Preset Objects.

\section{Completing for Rendering by:}

- Defining the position where the Specified Presentation will be implemented in the environment.

- Defining the shape-changing actuations that can be used for the Active Objects.

- Defining the light sources that can be applied to Active Lights.

- Defining the fabrication methods of the Specified Presentation.

\section{Completing for Interfacing by:}

- Defining the specific Controls for the added Interaction Loops.

- Defining the specific Interfaces for the added Interaction Loops.

- Defining the specific sensors that are needed to realize the added

Interaction Loops.

- Defining the specific update of the altered data transformation steps when the added Interaction Loops happen. 


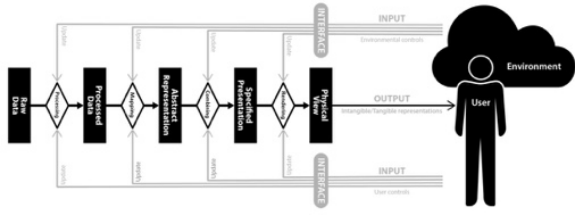

At the end of this step, we recommend designers to reflect on their completed concepts in three criteria presented in the table below.

\section{Functionality.}

This criterion concerns the context-specific requirements that SLS have to achieve. Designers can set this criterion by themselves according to the design brief.

\section{Feasibility.}

What are the possible limitations of the concepts in the aspects of technology, material, and fabrication.

-What are the possible constraints from the budget, the time, and the regulations.

\section{Extra Values.}

This criterion considers the cost-benefit ratio of the concepts of SLS. In some cases, a concept of SLS might be expensive but worth to be implemented if it brings extra value beyond expectation. For example, a concept of SLS involves some sensors to turn on/off the Information Decoration. Although implementing these sensors needs extra cost, it can also bring values of reducing energy consumption.

These reflections will help designers to make rational decisions before diving into the prototyping phase. The decision might be implementing the best concepts or improving some other concepts in another iteration of idea generation.

Now, please reflect on your completed concepts and highlight the best one on page 50 .

\section{Questionnaire \&Comment 3}

After the practice session, we would like to collect some feedback from you on the value of our Design Guidelines of SLS. Please rate in the below questionnaire. Also, we will appreciate it if you can leave a comment to explain each of your rates with a few words.

3.1. I do understand the explanation of the Design Guidelines of SLS.

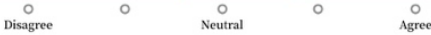

3.2. I think the Design Guidelines of SIS are also understandable for other designers.

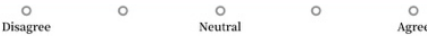

$52 / 53$ 


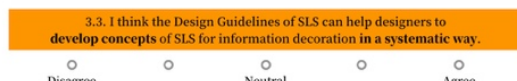

$\stackrel{\circ}{\text { Disagree }}$

Neutro

Agree

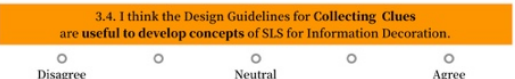

3.5. I think the Interaction Model of SLS can help designers to collect useful clues to develop concepts of SLS for information decoration.

$\begin{array}{cccc}\circ & \circ & 0 & 0 \\ 0 & & 0\end{array}$

$53 / 55$

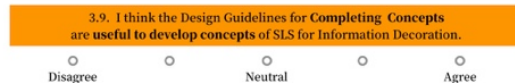

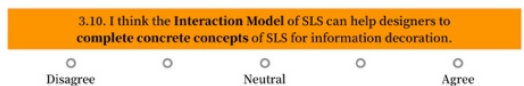

3.11. I think the Design Guidelines of SLS can help designers to reflect on their concepts of SLS for information decoration in a structural way.

$\stackrel{\circ}{\circ}$

Agree
3.6. I think the Design Guidelines for Generating Ideas useful to develop concepts of SLS for Information Decoration.

$\circ$

$\circ$

$\circ$
3.7. I think the Framework of SLS can help designers to
generate variety ideas to compose concepts of SLS for Information Decoration.

$\stackrel{\circ}{\circ}$

$\circ$

○
3.8. I think the Interaction Model of SLS can help designers to generate variety ideas to compose concepts of SLS for Information Decoration.

$\stackrel{\circ}{\text { Disagre }}$

$\stackrel{\circ}{\text { Neutral }}$

○
$54 / 55$ 


\section{About Author}

\section{Curriculum Vitae:}

Jiang Wu was born on 23-08-1986 in Henan China. In September 2006, he became a bachelor student in the Department of Industrial Design, Art and Design college at Nanchang University, China. After graduated in July 2010, he started his master program in the Department of Industrial Design, Computer and Science College at Zhejiang University, China. During his master period, Jiang continued developing his skills as a product designer and took part in a number of commercial design projects. Meanwhile, his design work has received several international design awards, such as four times of Red Dot Design Award, two of which are honoured as Best of the Best (2011 and 2012), and an iF Design Award (2010). In September 2013, Jiang started his PhD research in the Department of Industrial Design at Eindhoven University of Technology, the Netherlands. With a multidisciplinary background of fine art, product design, and computer science, he focused on the study of designing intelligent lighting systems that visualise information in a subtle and interactive way. The study is presented in this dissertation under the title of Designing Sculpting Light System for Information Decoration. Resulted from this $\mathrm{PhD}$ study, his work was exhibited in DDW (Dutch Design Week) 2015 and TEI (International Conference on Tangible, Embedded and Embodied Interaction) 2016.

\section{Publication \& Exhibition :}

Wu, J., Van Essen, H., \& Eggen, B. (2016). Designing Sculpting Light Systems for Information Decoration. In TEI 2016 - Proceedings of the 10th Anniversary Conference on Tangible Embedded and Embodied Interaction. https://doi.org/10.1145/2839462.2856547

Wu, J. 2015, Time Glow, Dutch Design Week, 17 October - 13 November 2015, VDMA, Eindhoven, The Netherlands. 


\section{Summary}

Natural light creates subtle, beautiful and expressive ambient displays through interacting with our physical world. The sparkling lake shows the blowing wind, the phase of the moon indicates the date during a month, and the shadow of the sun presents the time during a day. However, in the case of ambient displays by means of artificial light, we are more focus on manipulating the properties of light itself (e.g., colour or intensity), the features of the physical environment are rarely involved. Therefore, we propose SLS (Sculpting Light Systems), which combine shape-changing techniques with light to create ambient light systems that are aesthetically pleasing, subtly meaningful, tangibly interactive, and environmentally appropriated. SLS indicate a novel design space to designing ambient light systems that are featured with Information Decoration. The design-relevant knowledge of it is still lacking to support designers developing SLS in design practice. To fill this gap, we explored the design space of SLS by three main steps. Firstly, we gained an initial understanding of SLS by two hands-on design explorations (see Chapter 2), as well as a review of state-of-art examples of SLS (see Chapter 3). It helped us to reveal six design challenges of SLS and a potential way of applying SLS for adaptive Information Decoration. Also, it enabled us to formulate a Framework for SLS (see Chapter 4), which provides a systematic overview of the design alternatives in implementing SLS. They include: five basic components for construction, three types of attributes for manipulation, and three kinds of light patterns for information mapping. Secondly, we propose an Interaction Model for SLS (see Chapter 5) to provide designers with a coherent picture of what is possible when developing an adaptive SLS for Information Decoration. This Interaction Model consists of an information flow and four possible means of interaction loops, which support designers a systematic approach rather than anecdotical inspirations from designing SLS. Finally, we integrated the use of the SLS Framework and the SLS Interaction Model into a set of design guidelines (see Chapter 6) to support designers in developing SLS specifically in the ideation phases. By evaluating the design- relevant knowledge presented in this thesis through a user study (see Chapter 7), we found out that our Framework, Interaction Model, and SLS Design Guidelines are useful in ideating SLSconcepts. Moreover, this design-relevant knowledge can inspire design in the fields of interactive light installations, lighting interfaces, and shape-changing interfaces. 


\section{Acknowledgements}

Over the course of the $\mathrm{PhD}$ project that has led to this dissertation, many people have contributed to my work and supported me personally; I would like to take the opportunity to thank you all.

My most prominent thanks go to my promoter and supervisor for your continuous support in my PhD project. Berry, thank you for your patience, open-mindedness, and immense knowledge. You have always encouraged me to play to my strengths as a designer, meanwhile, kindly guided me to reflect on my work in depth as a researcher. That had a profound influence on me and has benefited me a lot in my current design career. Harm, thanks for being a strict but kindly mentor to me. I have learned so much from you, especially your proficient way of doing, and critical way of thinking.

Throughout my PhD project, I have been working in pleasant and supportive surroundings created by my office mates. Pencheng, thanks for your constructive suggestions about my study. Also, I would like to thank you for being a reliable friend to me in life. When I need any helps, you were always there. Serge, thank you for your kindly supports in my prototyping processes. Thomas, Saskia, Karin, thank you for many valuable advises in my research, and for many pleasant conversations or fun activities we had outside of work.

Besides my office mates, I am very grateful for having met so many great colleagues at ID TU/e, who have inspired me in different ways in my $\mathrm{PhD}$ research. Thanks to Jun $\mathrm{Hu}$, Yuan $\mathrm{Lu}$, Pengxiang Jia, Chao Wang, Linkai Tao, Bin Yu, Qi Wang, Brandon, Yaling Zhuang, Jesus, Kadian, Minna, Xipei Ren for sharing ideas that have helped my research. Many thanks to Yu Zhang, a fantastic artist and design researcher, who had always encouraged me to stick to my beliefs other than wants. My thanks also go to our considerate and responsive secretaries of the FE group, Rosalinde and Sabine. Moreover, I would like to thank Penny, Ali Zhao, Yudan Ma, Ning Zhang, Xu Lin, Kai Kang, Nan Yang, Hongyu Chen, Baisong Liu, Xinwei Wang and many other colleagues with whom I spent really wonderful time over the past few years.

In addition, I want to thank Xin Wang, Shuang Ma, Rishi, Yingzhe Wang, and Junquan Wei for the unforgettable moments that we shared together at gym. Importantly, I want to thank my family in China who have always supported me to finish my $\mathrm{PhD}$ even during the extremely hard time. 
By taking this opportunity, my special thanks to Jianhong, a true friend in my life, for every precious time that we have had together. Thank you for always being positive, optimistic, and supportive to me. I wish you success, and I know you will.

Last but not least, I would like to thank the reading committee of my dissertation, Dr. M. Graves-Petersen, Prof.dr. S. Luo, Prof. A.A.J. van Berlo and Dr. K. van Mensvoort, for devoting time and efforts into the review of my manuscript, and providing valuable comments for its improvement. 

